\title{
Lewis Base Catalyzed Aldol Additions of Chiral Trichlorosilyl Enolates and Silyl Enol Ethers
}

\author{
Scott E. Denmark, Shinji Fujimori and Son M. Pham \\ Department of Chemistry, University of Illinois Urbana-Champaign, Illinois 61801
}

\section{SUPPORTING INFORMATION}

Table of Contents

Page

General Experimental

Literature Preparations

Experimental Procedures

Preparation of TMS Enol Ethers

Aldol Addition of Trichlorosilyl Enolates

Aldol Addition of Trimethylsilyl Enol Ethers

References

${ }^{1} \mathrm{H}$ NMR Spectra of Trichlorosilyl Enolates

\section{General Experimental}

All reactions were performed in oven $\left(140^{\circ} \mathrm{C}\right)$ and/or flame-dried glassware under an atmosphere of dry argon, unless otherwise stated. All reaction temperatures correspond to internal temperatures measured by Teflon-coated thermocouples unless otherwise noted. Solvents for extraction and chromatography were technical grade and distilled from the indicated drying agents: dichloromethane $\left(\mathrm{CH}_{2} \mathrm{Cl}_{2}\right)$, pentane, hexane: $\mathrm{CaCl}_{2}$; ethyl acetate (EtOAc): $\mathrm{K}_{2} \mathrm{CO}_{3}$; acetone and methanol $(\mathrm{MeOH})$ were reagent grade. $n$-Butyllithium was titrated according to the method of Gilman. " "Brine" refers to a saturated aqueous solution of $\mathrm{NaCl}$.

${ }^{1} \mathrm{H},{ }^{13} \mathrm{C}$ and ${ }^{31} \mathrm{P}$ NMR spectra were recorded at $400 \mathrm{MHz}{ }^{1} \mathrm{H}, 100 \mathrm{MHz}{ }^{13} \mathrm{C}, 162 \mathrm{MHz}$ ${ }^{31} \mathrm{P}$ or $500 \mathrm{MHz}{ }^{1} \mathrm{H}, 125 \mathrm{MHz}{ }^{137} \mathrm{C}, 202 \mathrm{MHz}{ }^{31} \mathrm{P}$ in deuterochloroform unless otherwise stated. Data are reported in the following order: chemical shift in ppm $(\delta)$; multiplicities are indicated (br (broadened), s (singlet), d (doublet), t (triplet), q (quartet), m (multiplet)); coupling constants, 
$J$, are reported in hertz $(\mathrm{Hz})$; integration is provided; and assignment is indicated. ${ }^{1} \mathrm{H}$ and ${ }^{13} \mathrm{C}$ NMR assignments are corroborated by 2D experiments (HETCOR and COSY). Spectra are available on request from denmark@scs.uiuc.edu.

Low resolution electron impact (EI) spectra were performed at $70 \mathrm{eV}$, chemical ionization (CI) spectra were obtained using methane as the carrier gas. Data are reported in the form of $\mathrm{m} / z$ (intensity relative to base peak $=100$ ). Infrared spectra $(\mathrm{IR})$ are reported in $\mathrm{cm}^{-1}$ with indicated intensities: s (strong, $67-100 \%$ absorption), m (medium, $34-66 \%$ ), w (weak, 0 - 33\%). Optical rotation data was obtained on a digital polarimeter and are reported as follows: concentration ( $c=\mathrm{g} / 100 \mathrm{~mL}$ ), and solvent Analytical supercritical fluid chromatography (SFC) was performed using a built in photometric detector $(\lambda=220,258 \mathrm{~nm})$. Analytical columns used were Daicel Chiralpak AD and AS, Chiralcel OJ and OD and Regis $(R, R)$-Welk-01. Retention times $\left(t_{\mathrm{R}}\right)$ and peak areas for SFC were obtained from reporting integrators.

Analytical thin-layer chromatography was performed on Merck silica gel plates with F254 indicator. Visualization was accomplished by UV light, iodine, potassium permanganate solution $\left(\mathrm{KMnO}_{4}\right)$, phosphomolibdic acid stain (PMA), p-anisaldehyde stain or dinitrophenylhydrazine (DNP) solution. Column chromatography was performed by the method of Still ${ }^{2}$ with 32-63 mm silica gel (Merck). Bulb-to-bulb distillations were performed on a Kugelrohr and boiling points (bp) correspond to uncorrected, air bath temperatures. Melting points (mp) were determined in sealed capillary tubes and are corrected.

\section{Literature Preparations}

The following compounds were prepared by literature methods: The enolization using LiTMP followed the procedure described by Collum et al. ${ }^{3}$ The deprotection of TBS group followed the procedure described by Prakash et al. ${ }^{4}$ Diol 73 has been characterized by Albizati et al. ${ }^{5}$ The enolization using DBU to synthesize $(Z)$-enol ethers followed the procedure described by Yamaguchi, et. al. ${ }^{6}$ tert-Butyltritylamine (TBTA) was prepared according to the procedure described by Corey, et. al. ${ }^{7}$ 


\section{Experimental Procedures}

\section{(3S)-4-(tert-Butyldimethylsilanyloxy)-3-methyl-2-trimethylsilanyloxy-but-1-ene (8)}
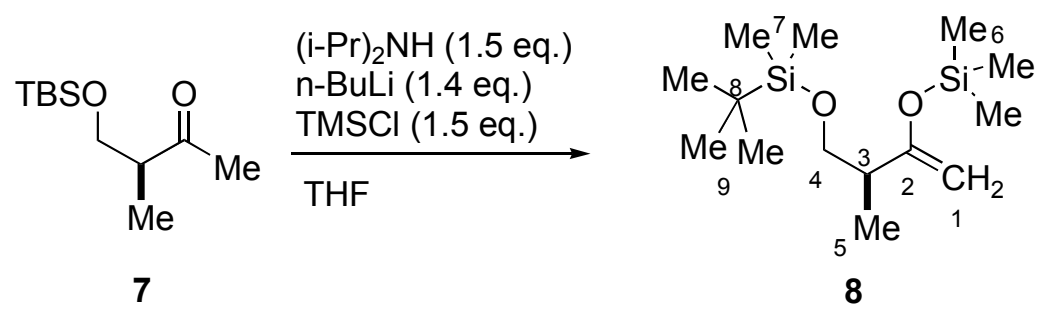

$n$-Butyllithium (1.54 M in hexane, $11.1 \mathrm{~mL}, 7.18 \mathrm{mmol}, 1.4$ equiv) was added dropwise to a solution of diisopropylamine $(1.67 \mathrm{~mL}, 11.9 \mathrm{mmol}, 1.5$ equiv) in $30 \mathrm{~mL}$ of tetrahydrofuran at $0^{\circ} \mathrm{C}$. The reaction mixture was stirred at $0{ }^{\circ} \mathrm{C}$ for $10 \mathrm{~min}$ then was cooled to $-78^{\circ} \mathrm{C}$. To the reaction mixture was added trimethylchlorosilane $(1.51 \mathrm{~mL}, 11.9 \mathrm{mmol}, 1.5$ equiv) and the ketone $7(1.71 \mathrm{~g}, 7.90 \mathrm{mmol})$ and the reaction mixture was stirred for $30 \mathrm{~min}$. The reaction mixture was gradually warmed up to $0{ }^{\circ} \mathrm{C}$ and was quenched with $40 \mathrm{~mL}$ of cold deionized water. The layers were separated and the aqueous layer was extracted twice with pentane. The combined extracts were washed with $50 \mathrm{~mL}$ of saturated aqueous copper sulfate, $25 \mathrm{~mL}$ of water and $50 \mathrm{~mL}$ of brine. The organic extracts were dried over sodium sulfate and were concentrated to give $2.32 \mathrm{~g}$ of crude oil. After distillation using a Vigreux column, the enolate 8 (1.94 g, 6.72 mmol, 85\%) was obtained as colorless oil.

Data for 8:

bp: $\quad 64{ }^{\circ} \mathrm{C}$ at $1.0 \mathrm{mmHg}$

${ }^{1}$ H NMR: $\quad\left(500 \mathrm{MHz}, \mathrm{CDCl}_{3}\right)$

4.05 (d, $J=0.7,1 \mathrm{H}, \mathrm{HC}(1)) ; 4.04$ (d, $J=0.7,1 \mathrm{H}, \mathrm{HC}(1)) ; 3.70-3.37$ (ABX, $2 \mathrm{H}$, $\mathrm{H}_{2} \mathrm{C}(4)$ ); 2.25 (sext, $J=6.6,1 \mathrm{H}, \mathrm{HC}(3)$ ); 1.01 (d, $J=6.8,3 \mathrm{H}, \mathrm{H}_{3} \mathrm{C}(5)$ ); 0.89 (s, 9 $\left.\mathrm{H}, \mathrm{H}_{3} \mathrm{C}(9)\right) ; 0.20$ (s, $\left.9 \mathrm{H}, \mathrm{H}_{3} \mathrm{C}(6)\right) ; 0.04$ (s, $6 \mathrm{H}, \mathrm{H}_{3} \mathrm{C}(7)$ )

${ }^{13} \mathrm{C} \mathrm{NMR}: \quad\left(125 \mathrm{MHz}, \mathrm{CDCl}_{3}\right)$

160.8 (C(2)); 89.4 (C(1)); 66.0 (C(4)); 43.1 (C(3)); 25.9 (C(9)); 18.3 (C(8)); 15.0 $(\mathrm{C}(5)) ; 0.1(\mathrm{C}(6)) ;-5.4(\mathrm{C}(7))$

IR: (neat)

3112 (w); 2958 (s); 2930 (s); 2898 (m); 2859 (m); 1657 (w); 1626 (m); 1471 (m); 1254 (s); 1088 (s); 1010 (s); 845 (s), 
MS: $\quad(\mathrm{FI})$

291 (11); 290 (26); $289\left(\mathrm{M}^{+}\right) ; 273$ (12); 231 (61); 145 (10); 115 (16); 73 (39)

Opt. rot. : $\quad-1.32\left(\mathrm{CHCl}_{3}, \mathrm{c}=2.5\right)$

Analysis: $\quad$ Calc for $\mathrm{C}_{14} \mathrm{H}_{32} \mathrm{O}_{2} \mathrm{Si}_{2}(288.57)$

Calc. $\quad$ C $58.27 \%$; H $11.18 \%$

Found: $\quad$ C $58.05 \%$; H $11.02 \%$

(3S)-4-(tert-Butyldimethylsilanyloxy)-3-methyl-2-trichlorosilanyloxy-but-1-ene (9)

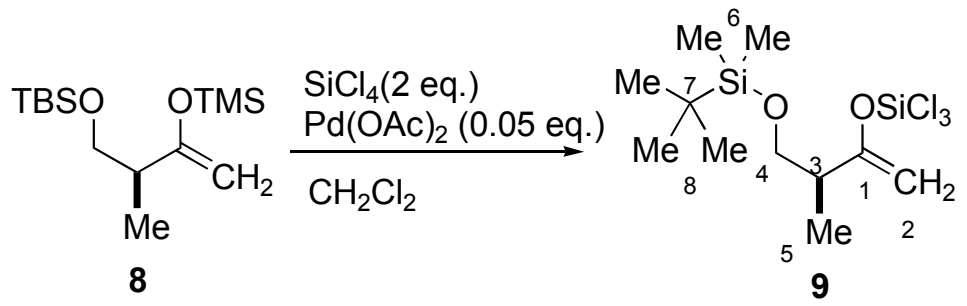

Silicon tetrachloride (1.74 mL, $15.1 \mathrm{mmol}, 2$ equiv) was added to a solution of palladium(II) acetate ( $85 \mathrm{mg}, 0.38 \mathrm{mmol}, 0.05$ equiv) in $7.57 \mathrm{~mL}$ of methylene chloride. Trimethylsilyl enol ether $8(2.18 \mathrm{~g}, 7.57 \mathrm{mmol})$ was added to the reaction mixture using a cannula, and the reaction mixture was stirred at $\mathrm{rt}$ for $1 \mathrm{~h}$. Trichlorosilyl enolate 9 (1.88 $\mathrm{g}, 5.37$ mmol, $71 \%$ ) was obtained by fractional distillation of the reaction mixture as a clear liquid.

Data for 9:

bp: $\quad 70-72{ }^{\circ} \mathrm{C}$ at $0.1 \mathrm{mmHg}$

${ }^{1}$ H NMR: $\quad(400 \mathrm{MHz})$

$4.58(\mathrm{~d}, J=2.4,1 \mathrm{H}, \mathrm{HC}(2)) ; 4.45(\mathrm{~d}, J=2.4,1 \mathrm{H}, \mathrm{HC}(2)) ; 3.68-3.46$ (ABX, $2 \mathrm{H}$, $\mathrm{H}_{2} \mathrm{C}(4)$ ); 2.39 (sext, $J=6.6,1 \mathrm{H}, \mathrm{HC}(3)$ ); 1.07 (d, $J=6.8,3 \mathrm{H}, \mathrm{H}_{3} \mathrm{C}(5)$ ); 0.89 (s, 9 $\left.\mathrm{H}, \mathrm{H}_{3} \mathrm{C}(8)\right) ; 0.04\left(\mathrm{~s}, 6 \mathrm{H}, \mathrm{H}_{3} \mathrm{C}(6)\right)$ 


\section{(4S)-4-(tert-Butyldimethylsilyloxy)-2-trimethylsilyloxy-1-pentene (11)}

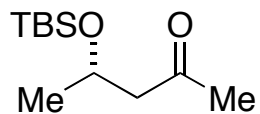

10

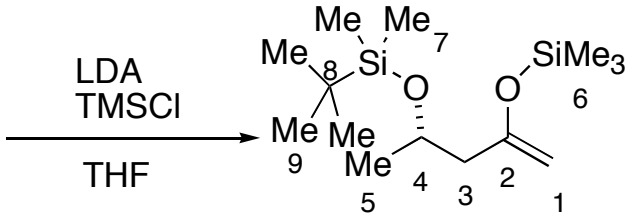

11

$n$-Butyllithium (1.54 M in hexane, $4.20 \mathrm{~mL}, 6.47 \mathrm{mmol}, 1.4$ equiv) was added dropwise to a solution of diisopropylamine $(0.971 \mathrm{~mL}, 6.93 \mathrm{mmol}, 1.5$ equiv) in $15 \mathrm{~mL}$ of tetrahydrofuran at $0^{\circ} \mathrm{C}$. The reaction mixture was stirred at $0{ }^{\circ} \mathrm{C}$ for $10 \mathrm{~min}$ then was cooled to $-78^{\circ} \mathrm{C}$. To the reaction mixture was added trimethylchlorosilane $(0.879 \mathrm{~mL}, 6.93 \mathrm{mmol}, 1.5$ equiv) and the ketone $10(1.00 \mathrm{~g}, 4.62 \mathrm{mmol})$ and the reaction mixture was stirred for $30 \mathrm{~min}$. The reaction mixture was gradually warmed up to $0{ }^{\circ} \mathrm{C}$ and was quenched with $20 \mathrm{~mL}$ of cold deionized water. The layers were separated and the aqueous layer was extracted twice with pentane. The combined extracts were washed with $20 \mathrm{~mL}$ of saturated aqueous copper sulfate, $15 \mathrm{~mL}$ of water and $20 \mathrm{~mL}$ of brine. The organic extracts were dried over sodium sulfate and were concentrated to give $1.61 \mathrm{~g}$ of crude oil. After distillation using a Kugelrohr apparatus, enol ether 11 (1.18 g, $4.09 \mathrm{mmol}, 89 \%$ ) was obtained as colorless oil.

Data for 11:

bp: $\quad 75^{\circ} \mathrm{C} \mathrm{ABT}$ at $0.5 \mathrm{mmHg}$

${ }^{1}$ H NMR: $\quad\left(500 \mathrm{MHz}, \mathrm{CDCl}_{3}\right)$

$4.04\left(\mathrm{~d}, J=7.6,2 \mathrm{H}, \mathrm{H}_{2} \mathrm{C}(1)\right)$; 4.04 (sext, $\left.J=6.5,1 \mathrm{H}, \mathrm{HC}(4)\right)$; 2.26-2.01 (ABX, 2

$\left.\mathrm{H}, \mathrm{H}_{2} \mathrm{C}(3)\right) ; 1.15$ (d, J=6.1, $\left.3 \mathrm{H}, \mathrm{H}_{3} \mathrm{C}(5)\right) ; 0.88$ (s, $\left.9 \mathrm{H}, \mathrm{H}_{3} \mathrm{C}(9)\right) ; 0.20$ (s, $9 \mathrm{H}$,

$\left.\mathrm{H}_{3} \mathrm{C}(6)\right) ; 0.05\left(\mathrm{~d}, J=1.9,6 \mathrm{H}, \mathrm{H}_{3} \mathrm{C}(7)\right)$

${ }^{13} \mathrm{C}$ NMR: $\quad\left(126 \mathrm{MHz}, \mathrm{CDCl}_{3}\right)$

156.7 (C(2)); 91.8 (C(1)); $66.4(\mathrm{C}(4)) ; 47.5$ (C(3)); 25.9 (C(9)); 23.4 (C(8)); 18.2 $(\mathrm{C}(5)) ; 0.1(\mathrm{C}(6)) ;-4.7,-4.8(\mathrm{C}(7))$

IR: (neat)

2959 (s); 2930 (m); 2898 (m); 2858 (m); 1660 (m); 1637 (m); 1464 (m); 1377

(m); 1304 (m); 1254 (s); 1211 (m); 1128 (m); 1087 (m); 1014 (m) 
$\underline{\mathrm{MS}}: \quad(\mathrm{FI})$

289 (61, M+); 273 (8); 254 (4); 231 (100); 199 (1); 185 (3); 159 (41); 131 (3); 115

(6); $73(40)$

Opt. Rot.: $\quad[\alpha]^{24}+6.81\left(\mathrm{CHCl}_{3}, \mathrm{c}=3.5\right)$

Analysis: $\quad$ Calc for $\mathrm{C}_{14} \mathrm{H}_{32} \mathrm{O}_{2} \mathrm{Si}_{2}(288.57)$
Calcd:
C, 58.27\%;
$\mathrm{H}, 11.18 \%$
Found:
C, $58.15 \%$;
$\mathrm{H}, 11.41 \%$

(4S)-4-Triisopropylsilyloxy-2-trimethylsilyloxy-1-pentene (36)

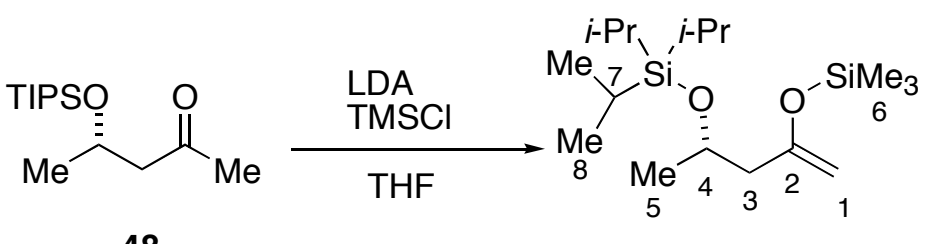

48

$n$-Butyllithium (1.54 M in hexane, $2.29 \mathrm{~mL}, 3.53 \mathrm{mmol}, 1.4$ equiv) was added dropwise to a solution of diisopropylamine $(0.53 \mathrm{~mL}, 3.78 \mathrm{mmol}, 1.5$ equiv) in $10 \mathrm{~mL}$ of tetrahydrofuran at $0^{\circ} \mathrm{C}$. The reaction mixture was stirred at $0{ }^{\circ} \mathrm{C}$ for $10 \mathrm{~min}$ then was cooled to $-78^{\circ} \mathrm{C}$. To the reaction mixture was added trimethylchlorosilane $(048 \mathrm{~mL}, 3.78 \mathrm{mmol}, 1.5$ equiv) and the ketone $48(651 \mathrm{mg}, 2.52 \mathrm{mmol})$ and the reaction mixture was stirred for $30 \mathrm{~min}$. The reaction mixture was gradually warmed up to $0{ }^{\circ} \mathrm{C}$ and was quenched with $20 \mathrm{~mL}$ of cold deionized water. The layers were separated and the aqueous layer was extracted twice with pentane. The combined extracts were washed with $20 \mathrm{~mL}$ of saturated aqueous copper sulfate, $15 \mathrm{~mL}$ of water and $20 \mathrm{~mL}$ of brine. The organic extracts were dried over sodium sulfate and were concentrated to give $1.61 \mathrm{~g}$ of crude oil. After distillation using a Kugelrohr apparatus, the enol ether 36 (688 mg, $2.08 \mathrm{mmol}, 83 \%$ ) was obtained as colorless oil.

Data for 36:

bp: $\quad 125^{\circ} \mathrm{C} \mathrm{ABT}$ at $1.0 \mathrm{mmHg}$

${ }^{1}$ H NMR: $\quad\left(500 \mathrm{MHz}, \mathrm{CDCl}_{3}\right)$

$4.12(\operatorname{sextd}, J=6.5,1.5,1 \mathrm{H}, \mathrm{HC}(4)) ; 4.04$ (d, $\left.J=11.7,2 \mathrm{H}, \mathrm{H}_{2} \mathrm{C}(1)\right) ; 2.38-2.02$

$\left(\mathrm{ABX}, 2 \mathrm{H}, \mathrm{H}_{2} \mathrm{C}(3)\right) ; 1.18\left(\mathrm{~d}, J=6.1,3 \mathrm{H}, \mathrm{H}_{3} \mathrm{C}(5)\right) ; 1.06-1.01\left(\mathrm{~m}, 21 \mathrm{H}, \mathrm{H}_{3} \mathrm{C}(8)\right.$, 
$\mathrm{HC}(7)) ; 0.19\left(\mathrm{~d}, J=1.9,6 \mathrm{H}, \mathrm{H}_{3} \mathrm{C}(6)\right)$

${ }^{13} \mathrm{C} \mathrm{NMR}: \quad\left(126 \mathrm{MHz}, \mathrm{CDCl}_{3}\right)$

$156.9(\mathrm{C}(2))$; $91.6(\mathrm{C}(1)) ; 66.5(\mathrm{C}(4)) ; 47.8(\mathrm{C}(3)) ; 23.2(\mathrm{C}(5)) ; 18.1(\mathrm{C}(8)) ; 12.3$ $(\mathrm{C}(7)) ;-0.0(\mathrm{C}(6))$

IR: (neat)

2962 (s); 2945 (s); 2885 (m); 2868 (s); 1659 (w); 1624 (w); 1464 (w); 1378 (w); 1303 (m); 1254 (m); 1211 (w); 1126 (m); 1090 (m); 1014 (s)

MS: $\quad(\mathrm{FI})$

332 (21); $331\left(65, \mathrm{M}^{+}\right) ; 289$ (11); 288 (25); 287 (100); 201 (60); 73 (25)

Opt. Rot.: $\quad[\alpha]_{D}^{24}+11.5\left(\mathrm{CHCl}_{3}, \mathrm{c}=0.95\right)$

Analysis: $\quad \mathrm{C}_{17} \mathrm{H}_{38} \mathrm{O}_{2} \mathrm{Si}_{2}(288.57)$

Calcd: $\quad$ C, $61.75 \%$;, $11.58 \%$

Found: $\quad$ C, $61.79 \%$; $\quad$ H, $11.80 \%$

(4S)- 4-(tert-Butyldimethylsilyloxy)-2-trichlorosilyloxy-1-pentene (12)

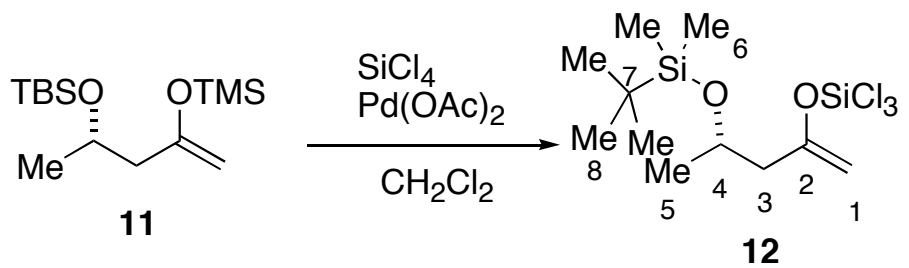

Silicon tetrachloride $(0.46 \mathrm{~mL}, 4.02 \mathrm{mmol}, 2.0$ equiv) was added to a solution of palladium(II) acetate $(22.6 \mathrm{mg}, 0.10 \mathrm{mmol}, 0.05$ equiv) in $2.00 \mathrm{~mL}$ of methylene chloride. Trimethylsilyl enol ether $11(580 \mathrm{mg}, 2.01 \mathrm{mmol})$ was added to the reaction mixture using a cannula, and the reaction mixture was stirred at $\mathrm{rt}$ for $30 \mathrm{~min}$. Trichlorosilyl enolate 12 (526 mg, $1.50 \mathrm{mmol}, 75 \%$ ) was obtained by Kugelrohr distillation of the reaction mixture as clear liquid.

Data for 12:

bp: $\quad 125^{\circ} \mathrm{C} \mathrm{ABT}$ at $1 \mathrm{mmHg}$

${ }^{1}$ H NMR: $\quad\left(400 \mathrm{MHz}, \mathrm{CDCl}_{3}\right.$ )

4.59 (d, $\left.J=1.9,1 \mathrm{H}, \mathrm{H}_{2} \mathrm{C}(1)\right) ; 4.46$ (d, $\left.J=2.0,1 \mathrm{H}, \mathrm{H}_{2} \mathrm{C}(1)\right) ; 4.04$ (sext, $J=6.2,1$ $\mathrm{H}, \mathrm{HC}(4))$; 2.35-2.16 (ABX, $2 \mathrm{H}, \mathrm{H}_{2} \mathrm{C}(3)$ ); 1.18 (d, J = 5.8, $3 \mathrm{H}, \mathrm{H}_{3} \mathrm{C}(5)$ ); 0.88 (s, 
$9 \mathrm{H}, \mathrm{H}_{3} \mathrm{C}(8) ; 0.06\left(\mathrm{~d}, J=3.6,6 \mathrm{H}, \mathrm{H}_{3} \mathrm{C}(6)\right)$

(4S)-(2Z)-3-Trimethylsilyloxy-4-tert-butyldimethylsilyloxy-pent-2-ene ((Z)-14)

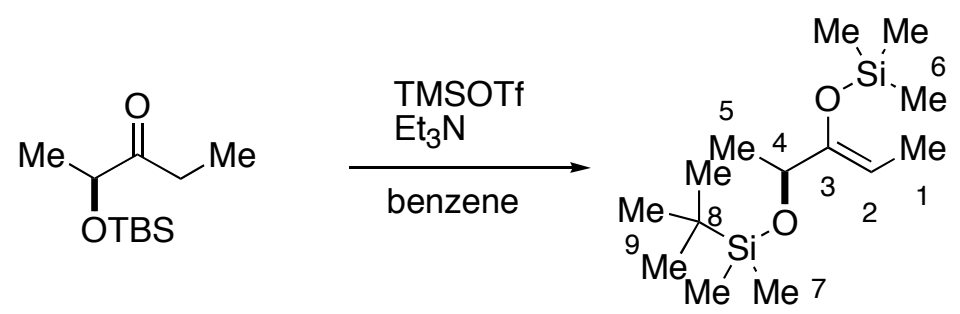

Trimethylsilyl trifluoromethanesulfonate (TMSOTf) $(240 \mu \mathrm{L}, 1.32 \mathrm{mmol}, 1.2 .0$ equiv) was dissolved in benzene $(2 \mathrm{~mL})$ at room temperature. Triethylamine $(210 \mu \mathrm{L}, 1.65 \mathrm{mmol}, 1.5$ equiv) was carefully added via syringe and the entire solution was cooled in an ice bath. Silyloxy ketone 13 (232 mg, $1.1 \mathrm{mmol}$ ) was then added dropwise via syringe. The reaction was allowed to warm to room temperature and monitored by TLC. After $3 \mathrm{~h}$, the biphasic mixture was quickly poured in to cold water $\left(10 \mathrm{~mL}, 0{ }^{\circ} \mathrm{C}\right)$ with rapid stirring. The layers were separated and the aqueous phase was extracted with pentane $(3 \times 5 \mathrm{~mL})$. The combined organic extracts were then washed with brine $(5 \mathrm{~mL})$, dried over $\mathrm{Na}_{2} \mathrm{SO}_{4}$, filtered and concentrated to give a crude oil. The residue was distilled under reduced pressure to afford $288 \mathrm{mg}(96 \%)$ of 14 as a clear, colorless oil, suitable for use in subsequent reactions. To obtain an analytically pure sample, 14 was sacrificially purified by chromatography $\left(\mathrm{SiO}_{2}\right.$, pentane $\left./ \mathrm{CH}_{2} \mathrm{Cl}_{2}, 6 / 1\right)$. The residue was again distilled under reduced pressure to afford $159 \mathrm{mg}(53 \%)$ of analytically pure 14.

Data for 14:

bp: $\quad 110^{\circ} \mathrm{C}(0.1 \mathrm{mmHg}, \mathrm{ABT})$

${ }^{1}$ H NMR: $\quad\left(\mathrm{CDCl}_{3}, 500 \mathrm{MHz}\right)$ $4.84(\mathrm{qd}, J=6.6,0.6,1 \mathrm{H}, \mathrm{HC}(4)) ; 4.08$ (q, $J=6.2,1 \mathrm{H}, \mathrm{HC}(2)) ; 1.54(\mathrm{dd}, J=$ 6.6, 0.9, $\left.3 \mathrm{H}, \mathrm{H}_{3} \mathrm{C}(5)\right) ; 1.25$ (d, $\left.J=6.2,3 \mathrm{H}, \mathrm{H}_{3} \mathrm{C}(1)\right)$; 0.92 (s, $9 \mathrm{H}, \mathrm{H}_{3} \mathrm{C}(9)$ ); 0.23 (s, $\left.9 \mathrm{H}, \mathrm{H}_{3} \mathrm{C}(6)\right) ; 0.08,\left(\mathrm{~d}, J=4.1,6 \mathrm{H}, \mathrm{H}_{3} \mathrm{C}(7)\right)$

${ }^{13} \mathrm{C} \mathrm{NMR}: \quad\left(\mathrm{CDCl}_{3}, 125 \mathrm{MHz}\right)$ $153.5(\mathrm{C}(3)) ; 101.5(\mathrm{C}(2)) ; 70.5(\mathrm{C}(4)) ; 25.9(\mathrm{C}(5)) ; 22.6(\mathrm{C}(9)) ; 18.3(\mathrm{C}(8)) ; 10.5$ $(\mathrm{C}(1)) ; 0.7(\mathrm{C}(6)) ;-4.7(\mathrm{C}(7)) ;-5.0(\mathrm{C}(7))$

MS: $\quad(\mathrm{FI})$ $289\left(\mathrm{M}^{+}+1,27\right), 288\left(\mathrm{M}^{+}, 100\right), 231(4), 120(2)$ 
IR: (neat)

2958 (s), 2931 (s), 2858 (m), 1257 (s), 1119 (m), 1078 (m), 1049 (m), 837 (s), 777

(m)

Opt. Rot.: $\quad[\alpha]_{\mathrm{D}}^{23}-4.1^{\circ}\left(c=2.00, \mathrm{CHCl}_{3}\right)$

Analysis: $\quad \mathrm{C}_{14} \mathrm{H}_{32} \mathrm{O}_{2} \mathrm{Si}_{2}(288.58)$

Calculated: $\quad$ C, $58.27 ; \quad H, 11.18 \%$

Found: $\quad$ C, $58.11 ; \quad H, 11.31 \%$

(S)-(Z)-Trichloro[(1-((1-((dimethyl)-(1,1-dimethylethyl)silyl)oxy)ethyl)propenyl)-oxy]silane ((Z)-23)

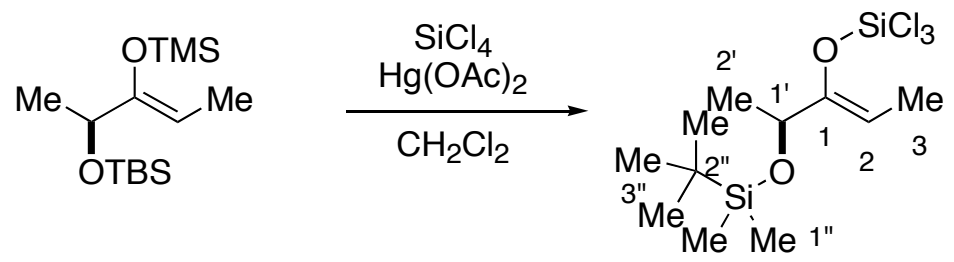

Mercuric acetate (32 mg, $0.1 \mathrm{mmol}, 0.01$ equiv) was suspended in $\mathrm{CH}_{2} \mathrm{Cl}_{2}(10 \mathrm{~mL})$ at room temperature. Silicon tetrachloride $(2.3 \mathrm{~mL}, 20 \mathrm{mmol}, 2.0$ equiv) was then carefully added via syringe and the cloudy mixture was allowed to stir for several minutes. TMS enol ether 14 $(2.7 \mathrm{~g}, 10 \mathrm{mmol})$ was then added dropwise via syringe. The reaction was allowed to stir at room temperature and could be monitored by careful removal of $10 \mu \mathrm{L}$ aliquots for ${ }^{1} \mathrm{H}$ NMR analysis. After $18 \mathrm{~h}$, the mercury salts were allowed to settle and the supernatant was carefully transferred to a dry $35 \mathrm{~mL}$ round bottom flask via cannula. The volatile components were removed at 100 $\mathrm{mmHg}$ and the residual oil was purified by distillation to afford $2.3 \mathrm{~g}(65 \%)$ of 23 as a $15 / 1$ mixture of $Z / E$ isomers by ${ }^{1} \mathrm{H}$ NMR.

Data for (Z)-23:

bp: $\quad 150{ }^{\circ} \mathrm{C}(0.1 \mathrm{mmHg}, \mathrm{ABT})$

${ }^{1}$ H NMR: $\quad\left(\mathrm{CDCl}_{3}, 500 \mathrm{MHz}\right)$

5.04 (q, $J=7.01 \mathrm{H}, \mathrm{HC}(2), Z) ; 4.95$ (q, $J=7.0,1 \mathrm{H}, \mathrm{HC}(2), E) ; 4.21$ (q, $J=6.8,1$ $\left.\mathrm{H}, \mathrm{HC}\left(1^{\prime}\right), Z\right) ; 4.18$ (q, $\left.J=6.8,1 \mathrm{H}, \mathrm{HC}\left(1^{\prime}\right), E\right) ; 1.60$ (d, $\left.J=7.0,3 \mathrm{H}, \mathrm{H}_{3} \mathrm{C}(3)\right)$; $1.31\left(\mathrm{~d}, J=6.8,3 \mathrm{H}, \mathrm{H}_{3} \mathrm{C}\left(2^{\prime}\right)\right) ; 0.93\left(\mathrm{~s}, 9 \mathrm{H}, \mathrm{H}_{3} \mathrm{C}\left(3^{\prime \prime}\right)\right) ; 0.07,(\mathrm{~d}, J=4.1,6 \mathrm{H}$, $\left.\mathrm{H}_{3} \mathrm{C}\left(1^{\prime \prime}\right)\right)$ 
${ }^{13} \mathrm{C}$ NMR: $\quad\left(\mathrm{CDCl}_{3}, 125 \mathrm{MHz}\right)$

$150.9(\mathrm{C}(1)) ; 108.3(\mathrm{C}(2)) ; 69.5\left(\mathrm{C}\left(1^{\prime}\right)\right) ; 25.8\left(\mathrm{C}\left(3^{\prime \prime}\right)\right) ; 22.2\left(\mathrm{C}\left(2^{\prime}\right)\right) ; 18.6\left(\mathrm{C}\left(2^{\prime \prime}\right)\right)$; $10.5(\mathrm{C}(3)) ; 0.7\left(\mathrm{C}\left(1^{\prime \prime \prime}\right)\right) ;-4.7\left(\mathrm{C}\left(1^{\prime \prime}\right)\right) ;-5.0\left(\mathrm{C}\left(1^{\prime \prime}\right)\right)$

\section{(-)-(S)-(E)-Trimethyl[(1-((1-((dimethyl)-(1,1-dimethylethyl)silyl)oxy)ethyl)-propenyl)oxy] silane $((E)-14)$}

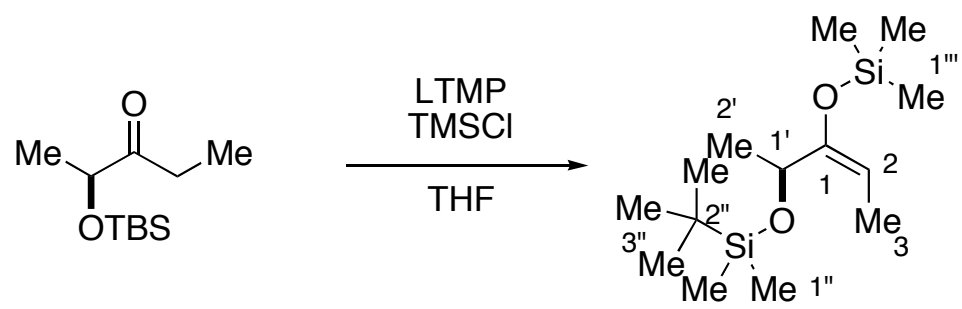

TMSCl (2.7 mL, $21.2 \mathrm{mmol}, 2$ equiv) was added via syringe to a solution containing LiTMP (13.8 mmol, 1.3 equiv) in THF $(25 \mathrm{~mL})$ at $-78{ }^{\circ} \mathrm{C}$. Ketone 13 (2.3 g, $10.6 \mathrm{mmol}, 1$ equiv) was then added dropwise over several minutes maintaining a temperature below $-65{ }^{\circ} \mathrm{C}$. Upon complete addition, the reaction was allowed to warm to room temperature and quenched with $\mathrm{H}_{2} \mathrm{O}(25 \mathrm{~mL})$. The reaction mixture was then washed with aqueous $\mathrm{CuSO}_{4}(3 \times 15 \mathrm{~mL})$, $\mathrm{H}_{2} \mathrm{O}(1 \times 15 \mathrm{~mL})$ and brine $(1 \times 15 \mathrm{~mL})$. The organic phase was dried over $\mathrm{Na}_{2} \mathrm{SO}_{4}$, filtered and concentrated in vacuo. Purification by column chromatography $\left(\mathrm{SiO}_{2}, 20 / 1\right.$ pentane $\left./ \mathrm{CH}_{2} \mathrm{Cl}_{2}\right)$ and distillation afforded $650 \mathrm{mg}(22 \%)$ of $(E)-\mathbf{1 4}$ as a clear, colorless oil.

Data for $(E)-\mathbf{1 4}$ :

bp: $\quad 110{ }^{\circ} \mathrm{C}(0.1 \mathrm{mmHg}, \mathrm{ABT})$

${ }^{1} \mathrm{H} \mathrm{NMR}: \quad\left(\mathrm{CDCl}_{3}, 500 \mathrm{MHz}\right)$

$5.01(\mathrm{qd}, J=7.0,0.6,1 \mathrm{H}, \mathrm{HC}(2)) ; 4.08\left(\mathrm{q}, J=6.2,1 \mathrm{H}, \mathrm{HC}\left(1^{\prime}\right)\right) ; 1.54(\mathrm{dd}, J=$ 6.6, 0.9, $\left.3 \mathrm{H}, \mathrm{H}_{3} \mathrm{C}(3)\right) ; 1.25$ (d, J = 7.0, $3 \mathrm{H}, \mathrm{H}_{3} \mathrm{C}\left(2^{\prime}\right)$ ); 0.92 (s, $9 \mathrm{H}, \mathrm{H}_{3} \mathrm{C}\left(3^{\prime \prime}\right)$ ); 0.23 (s, $\left.9 \mathrm{H}, \mathrm{H}_{3} \mathrm{C}\left(1^{\prime \prime \prime}\right)\right)$; 0.08, (d, $\left.J=4.1,6 \mathrm{H}, \mathrm{H}_{3} \mathrm{C}\left(1^{\prime \prime}\right)\right)$

${ }^{13} \mathrm{C} \mathrm{NMR}: \quad\left(\mathrm{CDCl}_{3}, 125 \mathrm{MHz}\right)$

$153.5(\mathrm{C}(1)) ; 101.5(\mathrm{C}(2)) ; 70.5\left(\mathrm{C}\left(1^{\prime}\right)\right) ; 25.9\left(\mathrm{C}\left(3^{\prime \prime}\right)\right) ; 22.6\left(\mathrm{C}\left(2^{\prime}\right)\right) ; 18.3\left(\mathrm{C}\left(2^{\prime \prime}\right)\right)$; 10.5 (C(3)); 0.7 (C(1"')); -4.7 (C(1")); -5.0 (C(1"))

MS: $\quad(\mathrm{FI})$

$288\left(\mathrm{M}^{+}, 100\right), 231(4), 120(2)$

IR: (neat)

2958 (s), 2931 (s), 2858 (m), 1257 (s), 1119 (m), 1078 (m), 1049 (m), 837 (s), 777 
(m)

Opt. Rot.: $\quad[\alpha]_{\mathrm{D}}^{23}-4.8^{\circ}\left(c=2.00, \mathrm{CHCl}_{3}\right)$

Analysis: $\quad \mathrm{C}_{14} \mathrm{H}_{32} \mathrm{O}_{2} \mathrm{Si}_{2}(288.58)$
Calculated:
C, 58.27;
$\mathrm{H}, 11.18 \%$
Found:
C, 58.15;
$\mathrm{H}, 11.25 \%$

(S)-(Z)-Trichloro[(1-((1-((dimethyl)-(1,1-dimethylethyl)silyl)oxy)ethyl)-propenyl)oxy]silane $((E)-23)$

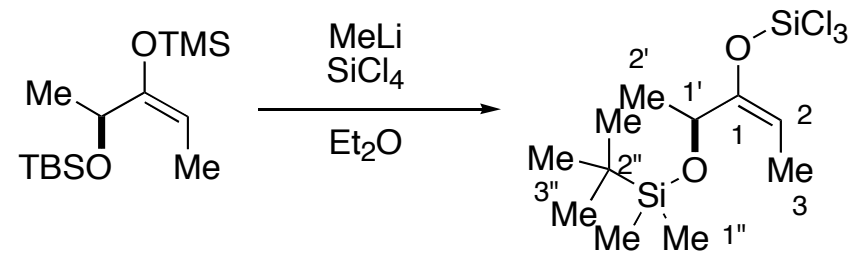

TMS enol ether $(E)-14(225 \mathrm{mg}, 1 \mathrm{mmol}, 1$ equiv) was added to a $10 \mathrm{~mL}, 2$ neck flask containing $\mathrm{Et}_{2} \mathrm{O}(3 \mathrm{~mL})$ cooled to $0{ }^{\circ} \mathrm{C}$ in an ice water bath. To this was added MeLi $(0.88 \mathrm{~mL}$, $1.2 \mathrm{mmol}, 1.2$ equiv) slowly via syringe. Upon complete addition, the reaction mixture was allowed to stir at $\mathrm{rt}$ for $6 \mathrm{~h}$. The contents of the reaction flask were then transferred dropwise via cannula to a solution containing $\mathrm{SiCl}_{4}\left(1.1 \mathrm{~mL}, 10 \mathrm{mmol}, 10\right.$ equiv) in $\mathrm{Et}_{2} \mathrm{O}(3 \mathrm{~mL})$ at $-78{ }^{\circ} \mathrm{C}$. The cryobath was removed and upon warming, the presence of a white precipitate was observed. The contents of the reaction were transferred via cannula to a clean flask, concentrated carefully in vacuo to afford $238 \mathrm{mg}(68 \%)$ of $(E)-\mathbf{2 3}$ as a colorless oil.

Data for $(E)-\mathbf{2 3}$ :

${ }^{1}$ H NMR: $\quad\left(\mathrm{CDCl}_{3}, 500 \mathrm{MHz}\right)$

$5.14(\mathrm{q}, J=7.01 \mathrm{H}, \mathrm{HC}(2), Z) ; 4.95$ (q, $J=7.0,1 \mathrm{H}, \mathrm{HC}(2), E) ; 4.21$ (q, $J=6.8,1$ $\left.\mathrm{H}, \mathrm{HC}\left(1^{\prime}\right), Z\right) ; 4.18\left(\mathrm{q}, J=6.8,1 \mathrm{H}, \mathrm{HC}\left(1^{\prime}\right), E\right) ; 1.60\left(\mathrm{~d}, J=7.0,3 \mathrm{H}, \mathrm{H}_{3} \mathrm{C}(3)\right)$; $1.31\left(\mathrm{~d}, J=6.8,3 \mathrm{H}, \mathrm{H}_{3} \mathrm{C}\left(2^{\prime}\right)\right) ; 0.93\left(\mathrm{~s}, 9 \mathrm{H}, \mathrm{H}_{3} \mathrm{C}\left(3^{\prime \prime}\right)\right) ; 0.07,(\mathrm{~d}, J=4.1,6 \mathrm{H}$, $\left.\mathrm{H}_{3} \mathrm{C}\left(1^{\prime \prime}\right)\right)$ 


\section{(2Z,4S)- 5-(tert-Butyldimethylsilyloxy)-4-methyl-3-trimethylsilyloxy-2-pentene ((Z)-17)}

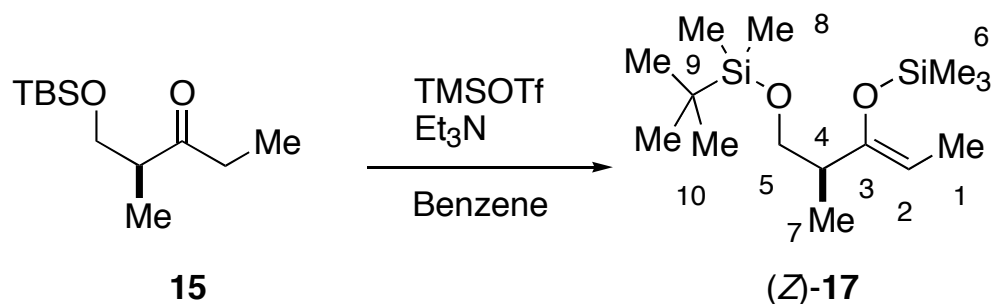

In a 50-mL two-neck flask was placed a solution of trimethylsilyl triflate (TMSOTf, 0.93 $\mathrm{mL}, 5.17 \mathrm{mmol}, 1.2$ equiv) in benzene $(15 \mathrm{~mL})$ at $0{ }^{\circ} \mathrm{C}$. To the solution were added sequentially triethylamine $(0.88 \mathrm{~mL}, 6.45 \mathrm{mmol}, 1.5$ equiv) and 15 (990 mg, $4.30 \mathrm{mmol})$. The reaction mixture was allowed to warm up to $\mathrm{rt}$ and stirred for $5 \mathrm{~h}$. The reaction mixture was cooled using an ice bath and was quenched with cold water $(15 \mathrm{~mL})$. The biphasic mixture was extracted with pentane $(2 \times 20 \mathrm{~mL})$, and the combined extracts were washed sequentially with saturated $\mathrm{CuSO}_{4}$ aq. $(2 \times 15 \mathrm{~mL})$, water $(10 \mathrm{~mL})$ and brine $(10 \mathrm{~mL})$. The solution was dried over sodium sulfate and concentrated to yield $1.42 \mathrm{~g}$ of crude oil. The crude oil was chromatographed (hexane $\left./ \mathrm{CH}_{2} \mathrm{Cl}_{2}, 8 / 1\right)$ to afford $100 \mathrm{mg}(0.33 \mathrm{mmol}, 8 \%)$ of $(E)-17$ and $688 \mathrm{mg}(2.27 \mathrm{mmol}$, $53 \%$ ) of (Z)-17 both as clear colorless oil. The analytically pure (Z)-17 was obtained by further distillation using the Kugelrohr apparatus $\left(125^{\circ} \mathrm{C} \mathrm{ABT}\right.$ at $\left.1.0 \mathrm{mmHg}\right)$.

Data for $(Z)-17$ :

bp: $\quad 170{ }^{\circ} \mathrm{C}(\mathrm{ABT})$ at $15 \mathrm{mmHg}$

${ }^{1} \mathrm{H}$ NMR: $\quad\left(500 \mathrm{MHz}, \mathrm{CDCl}_{3}\right)$

4.53 ( qd, $J=6.9,0.8,1 \mathrm{H}, \mathrm{HC}(2))$; 3.75-3.30 (ABX, $\left.2 \mathrm{H}, \mathrm{H}_{2} \mathrm{C}(5)\right)$ ) 2.20 (sext, $J=$ $6.1,1 \mathrm{H}, \mathrm{HC}(4)) ; 1.50\left(\mathrm{dd}, J=6.8,0.9,3 \mathrm{H}, \mathrm{H}_{3} \mathrm{C}(3)\right) ; 1.02(\mathrm{~d}, J=6.8,3 \mathrm{H}$, $\left.\mathrm{H}_{3} \mathrm{C}(6)\right) ; 0.88$ (s, $\left.9 \mathrm{H}, \mathrm{H}_{3} \mathrm{C}(10)\right)$; 0.20 (s, $\left.9 \mathrm{H}, \mathrm{TMS}\right) ; 0.03$ (s, $6 \mathrm{H}, \mathrm{H}_{3} \mathrm{C}(8)$ )

${ }^{13} \mathrm{C} \mathrm{NMR}: \quad\left(126 \mathrm{MHz}, \mathrm{CDCl}_{3}\right)$

152.9 (C(3)); $101.6(\mathrm{C}(2)) ; 66.4$ (C(5)); 43.1 (C(4)); 25.9 (C(10)); 18.3 (C(9)); $15.3(\mathrm{C}(7)) ; 10.9(\mathrm{C}(1)) ; 0.7(\mathrm{C}(6)) ;-5.4(\mathrm{C}(8))$

IR: (neat)

2958 (s); 2931 (s); 2860 (m); 1675 (m); 1472 (m); 1389 (w); 1319 (w); 1254 (s); 1191 (m); 1087 (s) 
MS: $\quad(\mathrm{FI})$

305 (2); 304 (12); 303 (31); 302.(100, M+); 245 (2); 199 (2

Opt. Rot.: $\quad[\alpha]_{\mathrm{D}}^{24}+1.50^{\circ}\left(\mathrm{CHCl}_{3}, \mathrm{c}=1.0\right)$

TLC: $\quad R_{f} 0.25$ (hexane $/ \mathrm{CH}_{2} \mathrm{Cl}_{2}, 5 / 1$ ) [silica gel, $\mathrm{KMnO}_{4}$ ]

Analysis: $\quad \mathrm{C}_{15} \mathrm{H}_{34} \mathrm{O}_{2} \mathrm{Si}_{2}(302.60)$

Calcd: $\quad$ C, $59.54 \%$; $\quad \mathrm{H}, 11.33 \%$

Found: $\quad$ C, $59.27 \%$; $\quad$ H, $11.52 \%$

\section{(2E,4S)- 5-(tert-Butyldimethylsilyloxy)-4-methyl-3-trimethylsilyloxy-2-pentene $((E)-17)$}

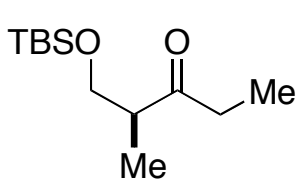

15

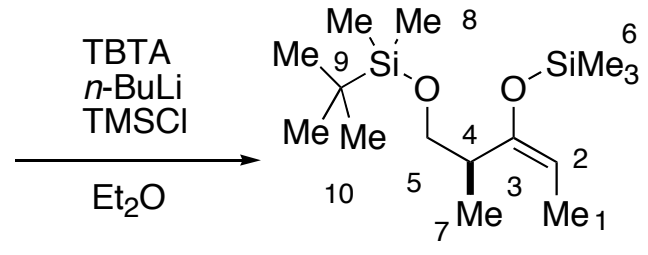

(E)-17

In a 100-mL two-neck flask was placed a solution of tert-butyl-tritylamine (TBTA, 2.05 $\mathrm{g}, 6.51 \mathrm{mmol}, 1.5$ equiv) in THF $(30 \mathrm{~mL})$ at $0{ }^{\circ} \mathrm{C}$. To the solution was added dropwise $n$-BuLi (3.95 mL, $6.08 \mathrm{mmol}, 1.4$ equiv, $1.54 \mathrm{M}$ in hexane). The solution was stirred at $0{ }^{\circ} \mathrm{C}$ for $10 \mathrm{~min}$ then cooled to $-72{ }^{\circ} \mathrm{C}$ using an acetone/dry ice bath. To the solution was added sequentially TMSCl (0.83 mL, $6.51 \mathrm{mmol}, 1.5$ equiv) and 15 (1.00 g, $4.34 \mathrm{mmol})$. The reaction mixture was stirred for $30 \mathrm{~min}$ at $-72{ }^{\circ} \mathrm{C}$, then gradually warmed up to $0^{\circ} \mathrm{C}$ over $1 \mathrm{~h}$. The reaction mixture was poured into cold deionized water $(15 \mathrm{~mL})$. The layers were separated and the aqueous layer was extracted using pentane $(2 \times 25 \mathrm{~mL})$. The combined organic extracts were washed with saturated $\mathrm{CuSO}_{4}$ aq. $(2 \times 10 \mathrm{~mL})$, water $(10 \mathrm{~mL})$ and brine $(10 \mathrm{~mL})$, dried over sodium sulfate and concentrated. The crude oil was purified by distillation using Kugelrhor $\left(175{ }^{\circ} \mathrm{C}\right.$ ABT at 15 $\mathrm{mmHg}$ ) followed by column chromatography (silica gel, hexane/ $\mathrm{CH}_{2} \mathrm{Cl}_{2}, 5 / 1,40 \mathrm{~mm}$ ) to afford $727 \mathrm{mg}(2.40 \mathrm{mmol}, 55 \%)$ of $(E)-\mathbf{1 7}$ as a clear oil and $68 \mathrm{mg}(0.22 \mathrm{mmol}, 5 \%, 1 / 1 \mathrm{Z} / \mathrm{E}$ mixture $)$ of $\mathbf{1 7}$ as colorless oil.

Data for $(E)-17$ :

bp: $\quad 150{ }^{\circ} \mathrm{C}(\mathrm{ABT})$ at $1.0 \mathrm{mmHg}$ 
${ }^{1} \mathrm{H}$ NMR: $\quad\left(500 \mathrm{MHz}, \mathrm{CDCl}_{3}\right)$

4.58 ( q, $J=6.8,1 \mathrm{H}, \mathrm{HC}(2))$; 3.60-3.38 (ABX, $2 \mathrm{H}, \mathrm{H}_{2} \mathrm{C}(5)$ ); 2.73 (sext, $J=7.1$, $1 \mathrm{H}, \mathrm{HC}(4)) ; 1.55$ (d, $\left.J=6.8,3 \mathrm{H}, \mathrm{H}_{3} \mathrm{C}(3)\right) ; 0.93$ (d, $\left.J=6.9,3 \mathrm{H}, \mathrm{H}_{3} \mathrm{C}(6)\right)$; 0.88 (s, $9 \mathrm{H}) ; 0.20$ (s, 9 H, TMS); 0.03 (s, $6 \mathrm{H})$

${ }^{13} \mathrm{C} \mathrm{NMR}: \quad\left(126 \mathrm{MHz}, \mathrm{CDCl}_{3}\right)$

153.0 (C(3)); 100.3 (C(2)); 65.8 (C(5)); 36.7 (C(4)); 25.9 (C(10)); 18.3 (C(9)); $14.1(\mathrm{C}(7)) ; 11.5(\mathrm{C}(1)) ; 0.4(\mathrm{C}(6)) ;-5.4,-5.4(\mathrm{C}(8))$

IR: (neat)

2958 (m); 2932 (m); 2898 (m); 2860 (m); 1667 (m); 1472 (m); 1388 (w); 1363 (w); 1253 (s); 1223 (s); 1105 (s); 1048 (m)

MS: $\quad(\mathrm{FI})$

304 (10); 303 (30); $302\left(100, \mathrm{M}^{+}\right) ; 199$ (2); 199 (2)

Opt. Rot.: $\quad[\alpha]_{\mathrm{D}}^{24}-8.99^{\circ}\left(\mathrm{CHCl}_{3}, \mathrm{c}=0.67\right)$

TLC: $\quad R_{f} 0.38$ (hexane $\left./ \mathrm{CH}_{2} \mathrm{Cl}_{2}, 5 / 1\right)$ [silica gel, $\mathrm{KMnO}_{4}$ ]

Analysis: $\quad \mathrm{C}_{15} \mathrm{H}_{34} \mathrm{O}_{2} \mathrm{Si}_{2}(302.60)$

Calcd: $\quad$ C, $59.54 \%$; $\quad \mathrm{H}, 11.33 \%$

Found: $\quad$ C, $59.54 \% ; \quad H, 11.63 \%$

(2Z,4S)- 5-(Triisopropylsilyloxy)-4-methyl-3-trimethylsilyloxy-2-pentene ((Z)-18)

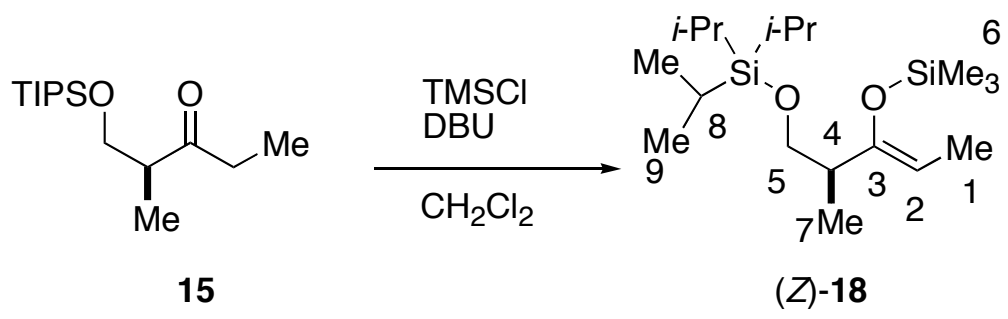

In a 100-mL round-bottom flask was placed a solution of TMSCl $(3.25 \mathrm{~mL}, 25.6 \mathrm{mmol}$, 1.2 equiv) in dichloromethane $(40 \mathrm{~mL})$ at $\mathrm{rt}$. To the solution were added DBU (3.84 mL, 25.6 mmol, 1.2 equiv) and $15(5.80 \mathrm{~g}, 21.3 \mathrm{mmol})$. The flask was fitted to a reflux condenser and the reaction mixture was refluxed for $4 \mathrm{~h}$. The reaction mixture was cooled using an ice bath and quenched with cold $\mathrm{NaHCO}_{3}$ aq. $(40 \mathrm{~mL})$. The product was extracted in pentane $(2 \mathrm{x} 50 \mathrm{~mL})$, 
and the combined extracts were washed with brine $(40 \mathrm{~mL})$. The organic extracts were dried over sodium sulfate and concentrated to yield $5.50 \mathrm{~g}$ of crude oil. The crude oil was chromatographed (silica gel, pentane $/ \mathrm{CH}_{2} \mathrm{Cl}_{2}, 5 / 1,50 \mathrm{~mm}$ ), and $5.03 \mathrm{~g}$ of (Z)-18 (14.6 mmol, $69 \%$ ) as clear colorless oil. Analytically pure sample of $(Z)-\mathbf{1 8}$ was obtained by distillation using the Kugelrohr apparatus.

Data for (Z)-18:

bp: $\quad 125{ }^{\circ} \mathrm{C}(\mathrm{ABT})$ at $0.05 \mathrm{mmHg}$

${ }^{1}$ H NMR: $\quad\left(500 \mathrm{MHz}, \mathrm{CDCl}_{3}\right)$

4.55 ( q, $J=6.7,1 \mathrm{H}, \mathrm{HC}(2)$ ); 3.85-3.38 (ABX, $2 \mathrm{H}, \mathrm{H}_{2} \mathrm{C}(5)$ ); 2.23 (sext, $J=6.3$, $1 \mathrm{H}, \mathrm{HC}(4)) ; 1.51$ (d, $\left.J=6.6,3 \mathrm{H}, \mathrm{H}_{3} \mathrm{C}(3)\right) ; 1.10$ - 1.03 (m, $24 \mathrm{H}, \mathrm{H}_{3} \mathrm{C}(7), \mathrm{H}_{3} \mathrm{C}(8)$, $\mathrm{HC}(8)) ; 0.19$ (s, $9 \mathrm{H}, \mathrm{TMS})$;

${ }^{13} \mathrm{C} \mathrm{NMR}: \quad\left(126 \mathrm{MHz}, \mathrm{CDCl}_{3}\right)$

$153.2(\mathrm{C}(3)) ; 101.9(\mathrm{C}(2)) ; 67.0(\mathrm{C}(5)) ; 43.6(\mathrm{C}(4)) ; 18.2(\mathrm{C}(9)) ; 15.6(\mathrm{C}(7)) ; 12.2$ $(\mathrm{C}(8)) ; 11.1(\mathrm{C}(1)) ; 0.9(\mathrm{C}(6))$;

IR: (neat) 2958 (s); 2931 (s); 2860 (m); 1675 (m); 1472 (m); 1389 (w); 1319 (w); 1254 (s); $1191(\mathrm{~m}) ; 1087(\mathrm{~s}) \backslash$

$\underline{\mathrm{MS}}: \quad(\mathrm{FI})$

347 (2); 346 (12); 345 (31); $344\left(100, \mathrm{M}^{+}\right)$;

Opt. Rot.: $\quad[\alpha]_{\mathrm{D}}^{24}+4.18^{\circ}\left(\mathrm{CHCl}_{3}, \mathrm{c}=2.5\right)$

TLC: $\quad R_{f} 0.33$ (pentane $\left./ \mathrm{CH}_{2} \mathrm{Cl}_{2}, 5 / 1\right)$ [silica gel, $\mathrm{KMnO}_{4}$ ]

Analysis: $\quad$ Calc for $\mathrm{C}_{18} \mathrm{H}_{40} \mathrm{O}_{2} \mathrm{Si}_{2}$ (344.68)
Calcd:
C, 62.72\%;
$\mathrm{H}, 11.70 \%$
Found:
C, 62.63\%;
H, $11.61 \%$ 
General Procedure I: MeLi Mediated Transsilylation of 17: $(2 Z, 4 S)$ - 5-(tertButyldimethylsilyloxy)-4-methyl-3-trichlorosilyloxy-2-pentene ((Z)-24)

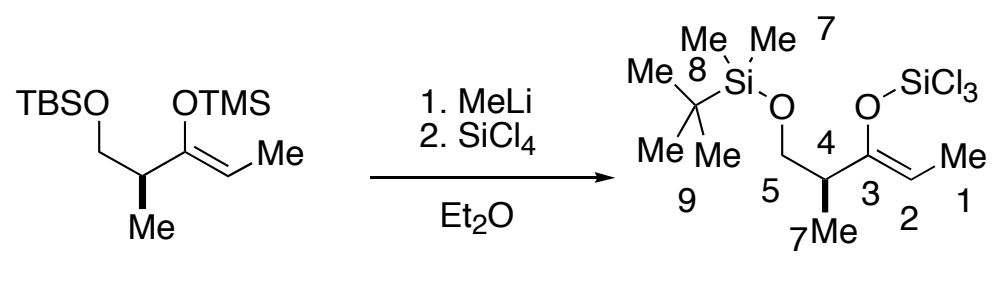

$(Z)-17$

$(Z)-24$

In a 25-mL two-neck flask was placed a solution of $(Z)-\mathbf{1 7}(908 \mathrm{mg}, 3.00 \mathrm{mmol})$ in ether $(6 \mathrm{~mL})$ at $0{ }^{\circ} \mathrm{C}$. To the solution was slowly added MeLi (3.00 mL, $4.50 \mathrm{mmol}, 1.5$ equiv, $1.5 \mathrm{M}$ in ether). The reaction mixture was stirred for $4.5 \mathrm{~h}$ at $\mathrm{rt}$, then cooled to $-70{ }^{\circ} \mathrm{C}$ using an dry ice/isopropanol bath. The reaction mixture was transferred dropwise using a cannula to a 100$\mathrm{mL}$ two-neck flask containing a cold $\left(-70{ }^{\circ} \mathrm{C}\right)$ solution of silicon tetrachloride $(3.45 \mathrm{~mL}, 30.0$ mmol, 10 equiv) in ether $(6 \mathrm{~mL})$. The reaction mixture was stirred at $-70{ }^{\circ} \mathrm{C}$ for $1 \mathrm{~h}$ and gradually warmed to rt. The precipitate settled at the bottom of the flask, and the supernatant was transferred to a $35-\mathrm{mL}$ round-bottom flask. The volatiles were removed under vacuum $(0.5$ $\mathrm{mmHg})$ and the residue was distilled using a Kugelrohr apparatus $\left(150{ }^{\circ} \mathrm{C} \mathrm{ABT}\right.$ at $\left.1.0 \mathrm{mmHg}\right)$, ato afford $901 \mathrm{mg}(2.48 \mathrm{mmol}, 83 \%)$ of (Z)-24 as a clear colorless oil.

Data for $(Z)-\mathbf{2 4}$ :

bp: $\quad 150{ }^{\circ} \mathrm{C}(\mathrm{ABT})$ at $1.0 \mathrm{mmHg}$

${ }^{1}$ H NMR: $\quad\left(\mathrm{CDCl}_{3}, 500 \mathrm{MHz}\right)$

4.53 (qd, $J=6.8,0.5,1 \mathrm{H}, \mathrm{HC}(2)$ ); 3.69-3.43 (ABX, $2 \mathrm{H}, \mathrm{H}_{2} \mathrm{C}(5)$ ); 2.40 (sext, $J=$ 6.3, $1 \mathrm{H}, \mathrm{HC}(4)) ; 1.60\left(\mathrm{dd}, J=7.1,0.7,3 \mathrm{H}, \mathrm{H}_{3} \mathrm{C}(1)\right) ; 1.07$ (d, $J=7.1,3 \mathrm{H}$, $\left.\mathrm{H}_{3} \mathrm{C}(6)\right) ; 0.88$ (s, $\left.9 \mathrm{H}, \mathrm{H}_{3} \mathrm{C}(9)\right) ; 0.03$ (s, $6 \mathrm{H}, \mathrm{H}_{3} \mathrm{C}(7)$ )

${ }^{13} \mathrm{C} \mathrm{NMR}: \quad\left(\mathrm{CDCl}_{3}, 126 \mathrm{MHz}\right)$

$150.8(\mathrm{C}(3)) ; 106.4(\mathrm{C}(2)) ; 65.3(\mathrm{C}(5)) ; 42.1(\mathrm{C}(4)) ; 25.8(\mathrm{C}(9)) ; 18.2(\mathrm{C}(8)) ; 14.9$ $(\mathrm{C}(7)) ; 11.0(\mathrm{C}(1)) ;-5.5,-5.4(\mathrm{C}(7))$; 
(2E,4S)- 5-(tert-Butyldimethylsilyloxy)-4-methyl-3-trichlorosilyloxy-2-pentene $((E)-24)$

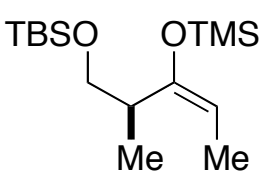

$(E)-17$

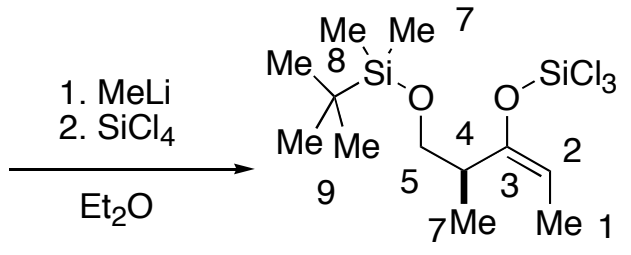

$(E)-24$

Following General Procedure I using (E)-17 (705 mg, $2.33 \mathrm{mmol})$, MeLi (2.33 mL, 3.49 mmol, 1.5 M solution in ether) and $\mathrm{SiCl}_{4}(2.67 \mathrm{~mL}, 23.3 \mathrm{mmol})$ in $7 \mathrm{~mL}$ of ether, $705 \mathrm{mg}(1.94$ mmol, $83 \%$ ) of (E)-24 was obtained as a colorless oil.

Data for $(E)-24:$

bp: $\quad 150{ }^{\circ} \mathrm{C}(\mathrm{ABT})$ at $1.0 \mathrm{mmHg}$

${ }^{1}$ H NMR: $\quad\left(\mathrm{CDCl}_{3}, 500 \mathrm{MHz}\right)$

$5.10(\mathrm{q}, J=7.1,1 \mathrm{H}, \mathrm{HC}(2)) ; 3.60-3.43\left(\mathrm{ABX}, 2 \mathrm{H}, \mathrm{H}_{2} \mathrm{C}(5)\right) ; 2.83$ (sext, $J=6.8,1$

$\mathrm{H}, \mathrm{HC}(4)) ; 1.61\left(\mathrm{~d}, J=6.8,3 \mathrm{H}, \mathrm{H}_{3} \mathrm{C}(1)\right) ; 0.99$ (d, $\left.J=6.8,3 \mathrm{H}, \mathrm{H}_{3} \mathrm{C}(6)\right) ; 0.88$ (s, 9

$\left.\mathrm{H}, \mathrm{H}_{3} \mathrm{C}(9)\right) ; 0.03\left(\mathrm{~d}, J=2.6,6 \mathrm{H}, \mathrm{H}_{3} \mathrm{C}(7)\right)$

${ }^{13} \mathrm{C} \mathrm{NMR}: \quad\left(\mathrm{CDCl}_{3}, 126 \mathrm{MHz}\right)$

$150.8(\mathrm{C}(3)) ; 106.7(\mathrm{C}(2)) ; 65.0(\mathrm{C}(5)) ; 36.4(\mathrm{C}(4)) ; 25.8(\mathrm{C}(9)) ; 18.2(\mathrm{C}(8)) ; 13.6$ $(\mathrm{C}(1)) ; 11.4(\mathrm{C}(6)) ;-5.5,-5.5(\mathrm{C}(7))$;

(2Z,4S)- 5-(Triisopropylsilyloxy)-4-methyl-3-trichlorosilyloxy-2-pentene ((Z)-25)

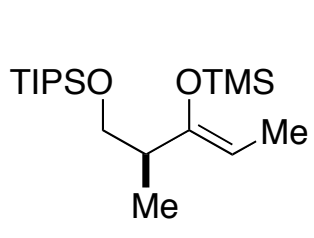

$(Z)-18$

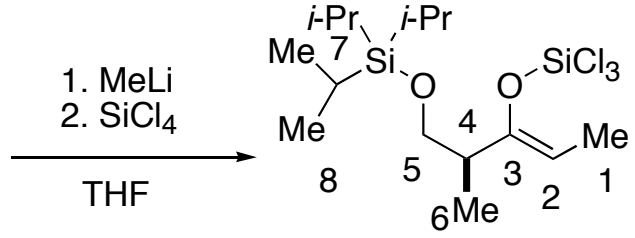

$(Z)-25$

Following General Procedure I using (Z)-18 (6.51 g, 18.9 mmol), MeLi (18.9 mL, 28.4 
mmol, 1.5 $\mathrm{M}$ in ether) and $\mathrm{SiCl}_{4}(21.7 \mathrm{~mL}, 189 \mathrm{mmol})$ in $50 \mathrm{~mL}$ of $\mathrm{THF}, 6.14 \mathrm{~g}(12.7 \mathrm{mmol}$, $67 \%)$ of (Z)-25 was obtained as a clear colorless oil.

Data for $(Z)-25:$

bp: $\quad 115^{\circ} \mathrm{C}$ at $1.0 \mathrm{mmHg}$

${ }^{1} \mathrm{H}$ NMR: $\quad\left(500 \mathrm{MHz}, \mathrm{CDCl}_{3}\right)$

4.85 (qd, $J=6.9,0.5,1 \mathrm{H}, \mathrm{HC}(2))$; 3.80-3.51 (ABX, $\left.2 \mathrm{H}, \mathrm{H}_{2} \mathrm{C}(5)\right)$ ) 2.43 (sext, $J=$ 5.8, $1 \mathrm{H}, \mathrm{HC}(4)) ; 1.60\left(\mathrm{dd}, J=6.9,0.9,3 \mathrm{H}, \mathrm{H}_{3} \mathrm{C}(3)\right) ; 1.10(\mathrm{~d}, J=6.9,3 \mathrm{H}$, $\left.\mathrm{H}_{3} \mathrm{C}(6)\right) ; 1.10-1.00\left(\mathrm{~m}, 21 \mathrm{H}, \mathrm{H}_{3} \mathrm{C}(8), \mathrm{HC}(7)\right)$;

${ }^{13} \mathrm{C} \mathrm{NMR}: \quad\left(126 \mathrm{MHz}, \mathrm{CDCl}_{3}\right)$

$164.8(\mathrm{C}(3)) ; 106.6(\mathrm{C}(2)) ; 65.9(\mathrm{C}(5)) ; 42.6(\mathrm{C}(4)) ; 18.2(\mathrm{C}(9)) ; 15.1(\mathrm{C}(7)) ; 12.2$ $(\mathrm{C}(8)) ; 11.3(\mathrm{C}(1))$;

\section{(2Z,5S)- 5-tert-Butyldimethylsilyloxy-3-trimethylsilyloxy-2-hexene ((Z)-21)}

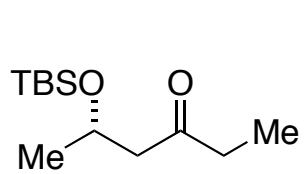

19

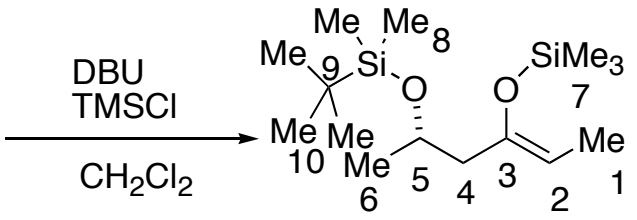

$(Z)-21$

In a $50 \mathrm{~mL}$ three-neck flask equipped with a stopper and a reflux condenser, DBU (1.30 $\mathrm{mL}, 8.68 \mathrm{mmol}, 2$ equiv) was dissolved in $20 \mathrm{~mL}$ of $\mathrm{CH}_{2} \mathrm{Cl}_{2}$. To this solution were added TMSCl (1.10 mL, $8.68 \mathrm{mmol}, 2$ equiv) and 19 (1.00 g, $4.34 \mathrm{mmol})$. The reaction mixture was heated to reflux for $4 \mathrm{~h}$. The reaction mixture was cooled to $\mathrm{rt}$ and quenched with cold sat. aq. $\mathrm{NaHCO}_{3}(20 \mathrm{~mL})$ solution The product was extracted with hexane $(2 \times 25 \mathrm{~mL})$ and washed with brine $(25 \mathrm{~mL})$. The extracts were dried over sodium sulfate and concentrated. The crude oil was chromatographed (silica gel, $5 / 1$ hexane $/ \mathrm{CH}_{2} \mathrm{Cl}_{2}, 40 \mathrm{~mm}$ ) and distilled using the Kugelrohr apparatus to give $820 \mathrm{mg}(2.71 \mathrm{mmol}, 62 \%)$ of (Z)-21 as clear colorless oil.

Data for $(Z)-21$ :

bp: $\quad 125{ }^{\circ} \mathrm{C}(0.5 \mathrm{mmHg})$ 
${ }^{1} \mathrm{H}$ NMR: $\quad\left(500 \mathrm{MHz}, \mathrm{CDCl}_{3}\right)$

4.54 (q, $J=6.6,1 \mathrm{H}, \mathrm{HC}(2)) ; 3.99$ (sext, $J=6.2,1 \mathrm{H}, \mathrm{HC}(4))$ ) 2.21-1.97 (ABX, 2

$\left.\mathrm{H}, \mathrm{H}_{2} \mathrm{C}(4)\right) ; 1.48$ (d, $\left.J=6.7,3 \mathrm{H}, \mathrm{H}_{3} \mathrm{C}(1)\right) ; 1.13$ (d, $\left.J=6.0,3 \mathrm{H}, \mathrm{H}_{3} \mathrm{C}(6)\right) ; 0.87$ (s,

$\left.9 \mathrm{H}, \mathrm{H}_{3} \mathrm{C}(10)\right) ; 0.18$ (s, $\left.9 \mathrm{H}, \mathrm{H}_{3} \mathrm{C}(7)\right) ; 0.03$ (d, , $J=6.2,6 \mathrm{H}, \mathrm{H}_{3} \mathrm{C}(8)$ )

${ }^{13} \mathrm{C} \mathrm{NMR}: \quad\left(126 \mathrm{MHz}, \mathrm{CDCl}_{3}\right)$

$148.5(\mathrm{C}(3)) ; 104.7(\mathrm{C}(2)) ; 66.4(\mathrm{C}(5)) ; 47.5(\mathrm{C}(4) ; 25.8$ (C(10)); $23.5(\mathrm{C}(9))$;

$18.1(\mathrm{C}(6)) ; 10.8(\mathrm{C}(1)) ; 0.6(\mathrm{C}(7)) ;-4.7,-4.9(\mathrm{C}(8))$

IR: (neat)

2958 (s); 2929 (m); 2899 (m); 2858 (m); 1680 (m); 1473 (m); 1377 (m); 1323

(m); 1254 (s); 1194 (m); 1128 (m); 1093 (m); 1070 (m); 1030 (m)

MS: $\quad(\mathrm{FI})$

$302\left(100, \mathrm{M}^{+}\right)$

Opt. Rot.: $\quad[\alpha]_{\mathrm{D}}^{24}+4.61\left(\mathrm{CHCl}_{3}, \mathrm{c}=0.97\right)$

TLC: $\quad R f 0.20$ (hexane/ $\left.\mathrm{CH}_{2} \mathrm{Cl}_{2}, 5 / 1\right)$ [silica gel, $\mathrm{KMnO}_{4}$ ]

Analysis: $\quad \mathrm{C}_{15} \mathrm{H}_{34} \mathrm{O}_{2} \mathrm{Si}_{2}(302.60)$

Calcd: $\quad$ C, $59.54 \% ; \quad H, 11.33 \%$

Found: $\quad$ C, $59.67 \%$;, $11.61 \%$

\section{(2Z,5S)- 5-Triisopropylsilyloxy-3-trimethylsilyloxy-2-hexene $((Z)-22)$}

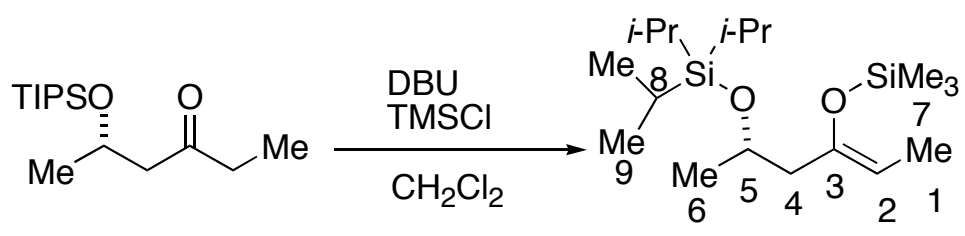

20

$(Z)-22$

In a $50 \mathrm{~mL}$ three-neck flask equipped with a stopper and a reflux condenser, DBU $(0.89$ $\mathrm{mL}, 6.06 \mathrm{mmol}, 1.5$ equiv) was dissolved in $15 \mathrm{~mL}$ of $\mathrm{CH}_{2} \mathrm{Cl}_{2}$. To this solution were added $\operatorname{TMSCl}(0.77 \mathrm{~mL}, 6.06 \mathrm{mmol}, 1.5$ equiv) and 20 (1.10 g, $4.04 \mathrm{mmol})$. The reaction mixture was heated to reflux for $4 \mathrm{~h}$. The reaction mixture was cooled to $\mathrm{rt}$ and quenched with $20 \mathrm{~mL}$ of cold sat. aq. $\mathrm{NaHCO}_{3}$ solution. The product was extracted in $50 \mathrm{~mL}$ of hexane and washed with 25 $\mathrm{mL}$ of brine. The extract was dried over sodium sulfate and concentrated. The crude product 
was chromatographed (silica gel, hexane $/ \mathrm{CH}_{2} \mathrm{Cl}_{2}, 8 / 1.40 \mathrm{~mm}$ ) and distilled using the Kugelrohr apparatus to afford $800 \mathrm{mg}(2.32 \mathrm{mmol}, 57 \%)$ of $(Z)-\mathbf{2 2}$ as clear colorless oil.

Data for $(Z)-22$ :

bp: $\quad 125^{\circ} \mathrm{C}(0.05 \mathrm{mmHg})$

${ }^{1}$ H NMR: $\quad\left(400 \mathrm{MHz}, \mathrm{CDCl}_{3}\right)$

$4.55(\mathrm{q}, J=6.8,1 \mathrm{H}, \mathrm{HC}(2)) ; 4.10$ (sextd, $J=5.6,2.7,1 \mathrm{H}, \mathrm{HC}(5)) ; 2.33-1.97$ (ABX, $\left.2 \mathrm{H}, \mathrm{H}_{2} \mathrm{C}(4)\right)$; 1.48 (d, $\left.J=6.8,3 \mathrm{H}, \mathrm{H}_{3} \mathrm{C}(1)\right) ; 1.17$ (d, $J=5.9,3 \mathrm{H}, \mathrm{H}_{3} \mathrm{C}(6)$ ); $1.16-1.05$ (m, $\left.21 \mathrm{H}, \mathrm{H}_{3} \mathrm{C}(9), \mathrm{HC}(8)\right) ; 0.18$ (s, $9 \mathrm{H}, \mathrm{H}_{3} \mathrm{C}(7)$ )

${ }^{13} \mathrm{C}$ NMR: $\quad\left(126 \mathrm{MHz}, \mathrm{CDCl}_{3}\right)$

148.7 (C(3)); 104.7 (C(2)); 66.7 (C(5)); 47.8 (C(4); 23.6 (C(6)); 18.1 (C(9)); 12.4 $(\mathrm{C}(8)) ; 10.9(\mathrm{C}(1)) ; 0.6(\mathrm{C}(7))$

IR: (neat)

2961 (s); 2944 (s); 2895 (m); 2867 (s); 1678 (m); 1464 (m); 1377 (m); 1323 (m); 1253 (s); 1193 (m); 1128 (m); 1096 (m); 1069 (m); 1028 (m); 1004 (m)

MS: $\quad(\mathrm{FI})$

$346(12) ; 345(33) ; 344\left(100, \mathrm{M}^{+}\right) ; 263$ (3)

Opt. Rot.: $\quad[\alpha]_{\mathrm{D}}^{24}+6.57\left(\mathrm{CHCl}_{3}, \mathrm{c}=0.48\right)$

TLC: $\quad R f 0.25$ (hexane/ $\mathrm{CH}_{2} \mathrm{Cl}_{2}, 8 / 1$ ) [silica gel, $\mathrm{KMnO}_{4}$ ]

Analysis: $\quad \mathrm{C}_{18} \mathrm{H}_{40} \mathrm{O}_{2} \mathrm{Si}_{2}(344.68)$

Calcd: $\quad$ C, $62.72 \% ; \quad \mathrm{H}, 11.70 \%$

Found: $\quad$ C, $62.53 \% ; \quad H, 12.00 \%$

\section{(2E,5S)- 5-Triisopropylsilyloxy-3-trimethylsilyloxy-2-hexene $((E)-22)$}

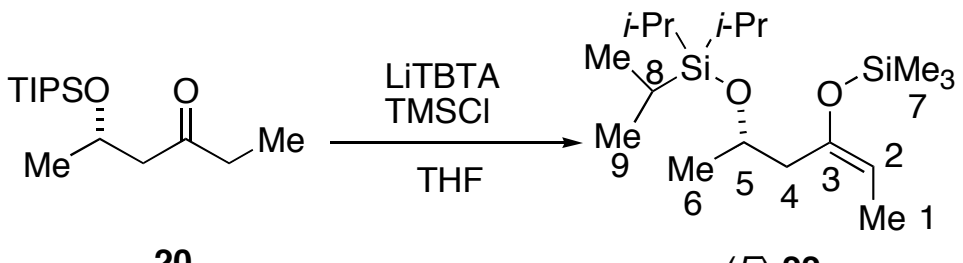

20

$(E)-22$

In a $25 \mathrm{~mL}$ two-neck flask equipped with a stopper and a septum, TBTA (946 mg, 3.00 mmol, 1.5 equiv) was dissolved in $10 \mathrm{~mL}$ of THF at $0{ }^{\circ} \mathrm{C}$. To this solution was added $n$-BuLi 
(1.82 mL, $2.80 \mathrm{mmol}, 1.4$ equiv, 1.54 $\mathrm{M}$ in hexane) dropwise, and the solution was stirred for 10 min. The solution was cooled to $-78{ }^{\circ} \mathrm{C}$, then $\mathrm{TMSCl}(0.381 \mathrm{~mL}, 3.00 \mathrm{mmol}, 1.5$ equiv) and 20 $(0.55 \mathrm{~g}, 2.00 \mathrm{mmol})$ were added. The reaction mixture was stirred for $30 \mathrm{~min}$ and gradually warmed to $0{ }^{\circ} \mathrm{C}$ over $2 \mathrm{~h}$. The reaction mixture was quenched with $10 \mathrm{~mL}$ of cold sat. aq. $\mathrm{NaHCO}_{3}$ solution. The product was extracted in $50 \mathrm{~mL}$ of pentane and washed with $25 \mathrm{~mL}$ of brine. The extract was dried over sodium sulfate and concentrated. The residue was redissolved in pentane $(60 \mathrm{~mL})$ and cooled to $-15{ }^{\circ} \mathrm{C}$ to crystallize TBTA. The recovered TBTA was removed by filtration and the filtrate was condensed under reduced pressure. The crude product was chromatographed (silica gel, hexane $/ \mathrm{CH}_{2} \mathrm{Cl}_{2}, 8 / 1,30 \mathrm{~mm}$ ) and distilled using the Kugelrohr apparatus to give $475 \mathrm{mg}(1.38 \mathrm{mmol}, 69 \%)$ of $(E)-22$ as clear colorless oil.

Data for $(E)-22:$

bp: $\quad 125^{\circ} \mathrm{C}(0.05 \mathrm{mmHg})$

${ }^{1} \mathrm{H}$ NMR: $\quad\left(400 \mathrm{MHz}, \mathrm{CDCl}_{3}\right)$

$4.67(\mathrm{q}, J=6.8,1 \mathrm{H}, \mathrm{HC}(2)) ; 4.14(\mathrm{~m}, 1 \mathrm{H}, \mathrm{HC}(5)) ; 2.32-2.23(\mathrm{ABX}, 2 \mathrm{H}$, $\left.\mathrm{H}_{2} \mathrm{C}(4)\right) ; 1.55\left(\mathrm{dd}, J=6.8,0.5,3 \mathrm{H}, \mathrm{H}_{3} \mathrm{C}(1)\right) ; 1.16\left(\mathrm{~d}, J=6.1,3 \mathrm{H}, \mathrm{H}_{3} \mathrm{C}(6)\right) ; 1.07$ (s, $\left.21 \mathrm{H}, \mathrm{H}_{3} \mathrm{C}(9), \mathrm{HC}(8)\right) ; 0.15$ (s, $9 \mathrm{H}, \mathrm{H}_{3} \mathrm{C}(7)$ )

${ }^{13} \mathrm{C}$ NMR: $\quad\left(126 \mathrm{MHz}, \mathrm{CDCl}_{3}\right)$

$149.6(\mathrm{C}(3)) ; 102.6(\mathrm{C}(2)) ; 67.0(\mathrm{C}(5)) ; 42.0(\mathrm{C}(4) ; 23.2(\mathrm{C}(6)) ; 18.1(\mathrm{C}(9)) ; 12.4$ $(\mathrm{C}(8)) ; 12.1(\mathrm{C}(1)) ; 0.2(\mathrm{C}(7))$

IR: (neat)

2962 (m); 2945 (m); 2895 (m); 2868 (m); 1670 (w); 1464 (m); 1379 (w); 1254 (m); 1198 (m); 1124 (m); 1107 (m); 1088 (m); 1005 (m)

MS: $\quad(\mathrm{FI})$

347 (2); 346 (11); 345 (30); 344 (100, $\left.\mathrm{M}^{+}\right) ; 344$ (2); 264 (2); 264 (3); 264 (5); 263

(3)

Opt. Rot.: $\quad[\alpha]_{\mathrm{D}}^{24}+6.64\left(\mathrm{CHCl}_{3}, \mathrm{c}=0.39\right)$

TLC: $\quad R f 0.26$ (hexane/ $\mathrm{CH}_{2} \mathrm{Cl}_{2}, 8 / 1$ ) [silica gel, $\mathrm{KMnO}_{4}$ ]

Analysis: $\quad \mathrm{C}_{18} \mathrm{H}_{40} \mathrm{O}_{2} \mathrm{Si}_{2}(344.68)$
Calcd:
C, $62.72 \%$;
H $11.70 \%$
Found:
C, 62.38\%;
$\mathrm{H}, 11.94 \%$ 
(2Z,5S)- 5-tert-Butyldimethylsilyloxy-3-trichlorosilyloxy-2-hexene ((Z)-26)

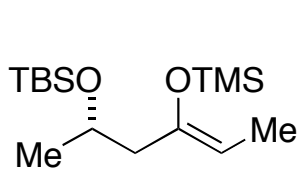

$(Z)-21$

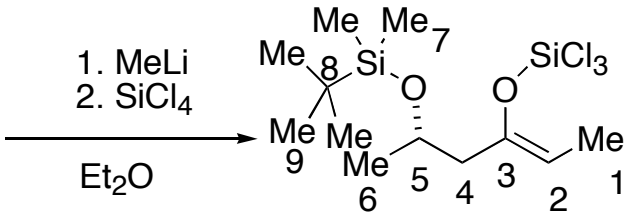

$(Z)-26$

Following the General Procedure I using (Z)-21 (972 mg, $3.21 \mathrm{mmol})$, MeLi (3.30 mL, $4.82 \mathrm{mmol}, 1.5$ equiv, $1.5 \mathrm{M}$ in ether) and silicon tetrachloride (3.68 mL, $32.1 \mathrm{mmol}, 10$ equiv), $764 \mathrm{mg}(2.10 \mathrm{mmol}, 65 \%)$ of (Z)-26 was obtained as a clear colorless oil.

Data for $(Z)-\mathbf{2 6}$ :

bp: $\quad 150{ }^{\circ} \mathrm{C}(\mathrm{ABT})$ at $1.0 \mathrm{mmHg}$

${ }^{1}$ H NMR: $\quad\left(\mathrm{CDCl}_{3}, 500 \mathrm{MHz}\right)$

4.83 (q, $J=6.9,1 \mathrm{H}, \mathrm{HC}(2))$; 4.03 (sext, $J=7.3,1 \mathrm{H}, \mathrm{HC}(5)$ ); 2.33-2.18 (ABX, 2 $\left.\mathrm{H}, \mathrm{H}_{2} \mathrm{C}(4)\right) ; 1.58$ (d, $\left.J=6.8,3 \mathrm{H}, \mathrm{H}_{3} \mathrm{C}(1)\right) ; 1.15$ (d, $\left.J=6.2,3 \mathrm{H}, \mathrm{H}_{3} \mathrm{C}(6)\right) ; 0.87$ (s, $\left.9 \mathrm{H}, \mathrm{H}_{3} \mathrm{C}(9)\right) ; 0.03$ (d, $\left.J=10.5,6 \mathrm{H}, \mathrm{H}_{3} \mathrm{C}(7)\right)$

(2Z,5S)- 5-Triisopropylsilyloxy-3-trichlorosilyloxy-2-hexene ((Z)-27)

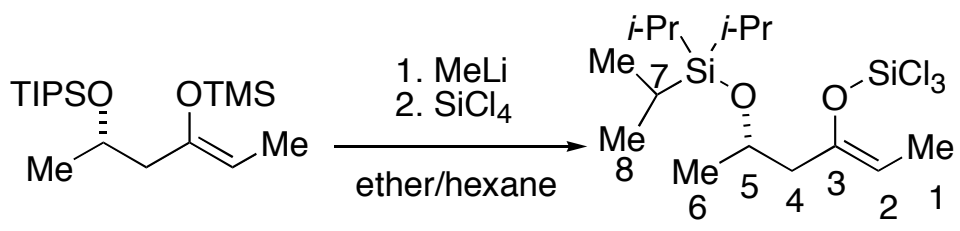

$(Z)-21$

$(Z)-27$

Following the General Procedure I using (Z)-22 (1.60 g, $4.64 \mathrm{mmol})$, MeLi (4.64 mL, $6.96 \mathrm{mmol}, 1.5 \mathrm{M}$ in ether) and $\mathrm{SiCl}_{4}(5.32 \mathrm{~mL}, 46.4 \mathrm{mmol})$ in $10 \mathrm{~mL}$ of hexane, $1.13 \mathrm{~g}(2.78$ mmol, $60 \%)$ of $(Z)-27$ was obtained as a clear colorless oil.

Data for $(Z)-\mathbf{2 7}$ :

bp: $\quad 175^{\circ} \mathrm{C}$ at $1.0 \mathrm{mmHg}$ 
${ }^{1} \mathrm{H}$ NMR: $\quad\left(500 \mathrm{MHz}, \mathrm{CDCl}_{3}\right)$

4.84 (q, $J=6.8,1 \mathrm{H}, \mathrm{HC}(2))$; 4.16 (sext, $J=7.1,1 \mathrm{H}, \mathrm{HC}(5)$ ); 2.47-2.17 (ABX, 2 $\left.\mathrm{H}, \mathrm{H}_{2} \mathrm{C}(4)\right) ; 1.59$ (dt, $\left.J=6.8,1.0,3 \mathrm{H}, \mathrm{H}_{3} \mathrm{C}(3)\right) ; 1.20$ (d, $\left.J=5.9,3 \mathrm{H}, \mathrm{H}_{3} \mathrm{C}(6)\right)$; $1.13-1.01\left(\mathrm{~m}, 21 \mathrm{H}, \mathrm{H}_{3} \mathrm{C}(8), \mathrm{HC}(7)\right)$;

(2E,5S)- 5-Triisopropylsilyloxy-3-trichlorosilyloxy-2-hexene ((E)-27)

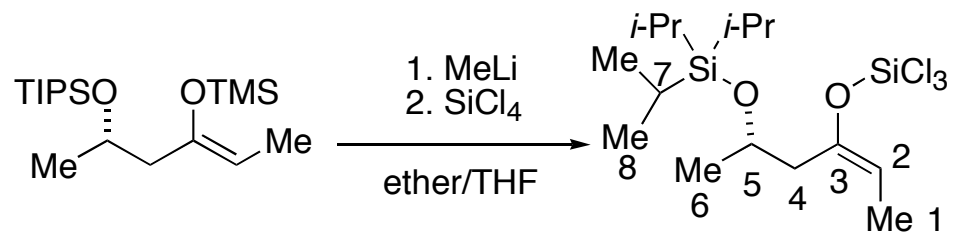

$(E)-22$

$(E)-27$

Following General Procedure I using (E)-22 (678 mg, $1.97 \mathrm{mmol})$, MeLi (1.57 mL, 2.36 mmol, $1.5 \mathrm{M}$ in ether) and $\mathrm{SiCl}_{4}(2.26 \mathrm{~mL}, 19.7 \mathrm{mmol})$ in $5 \mathrm{~mL}$ of ether, $180 \mathrm{mg}(0.44 \mathrm{mmol}$, $23 \%)$ of $(E)-27$ was obtained as a clear colorless oil.

Data for $(E)-27$ :

bp: $\quad 150{ }^{\circ} \mathrm{C}$ at $0.5 \mathrm{mmHg}$

${ }^{1}$ H NMR: $\quad\left(500 \mathrm{MHz}, \mathrm{CDCl}_{3}\right)$

$5.17(\mathrm{q}, J=7.1,1 \mathrm{H}, \mathrm{HC}(2)) ; 4.17(\mathrm{~m}, 1 \mathrm{H}, \mathrm{HC}(5)) ; 2.56-2.31(\mathrm{ABX}, 2 \mathrm{H}$, $\left.\mathrm{H}_{2} \mathrm{C}(4)\right) ; 1.62\left(\mathrm{~d}, J=7.1,3 \mathrm{H}, \mathrm{H}_{3} \mathrm{C}(3)\right) ; 1.20$ (d, $\left.J=6.1,3 \mathrm{H}, \mathrm{H}_{3} \mathrm{C}(6)\right) ; 1.09-1.02$ $\left(\mathrm{m}, 21 \mathrm{H}, \mathrm{H}_{3} \mathrm{C}(8), \mathrm{HC}(7)\right)$;

General Procedure II: Aldol Addition of Isolated Trichlorosilyl Enolates: (2S,5R)-1-tertButyldimethylsilyloxy-2-methyl-5-hydroxy-5-phenyl-3-pentanone (syn-28a)

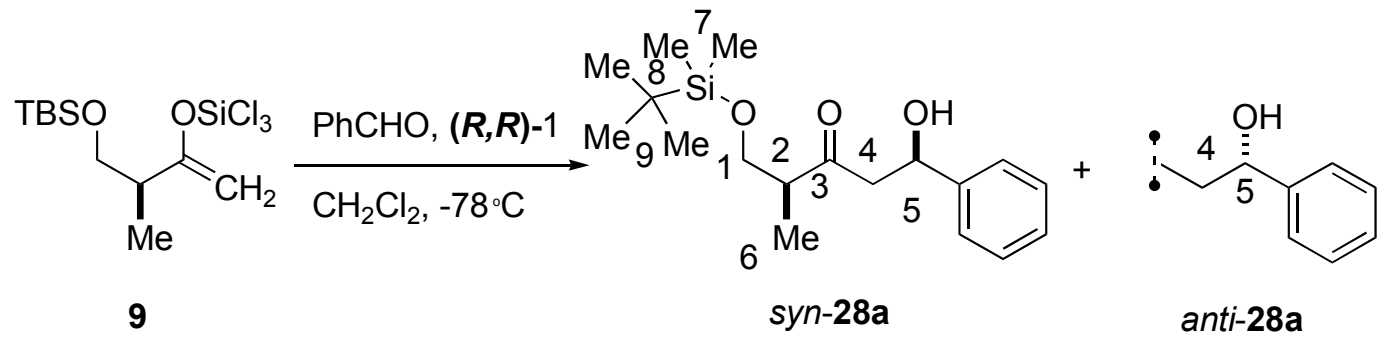

Trichlorosilyl enolate 9 (140 mg, $0.40 \mathrm{mmol}, 1.2$ equiv) was added to a solution of $(R, R)$ - 
1 (12.2 mg, $0.033 \mathrm{mmol}, 0.10$ equiv) in $0.66 \mathrm{~mL}$ of $\mathrm{CH}_{2} \mathrm{Cl}_{2}$. The reaction mixture was cooled using an acetone $/ \mathrm{CO}_{2}$ bath, and $\mathrm{PhCHO}(34 \mu \mathrm{L}, 0.33 \mathrm{mmol}, 1$ equiv) was added using a syringe. The reaction was monitored by taking an aliquot from the reaction mixture and analyzing on TLC (hexane/EtOAc, 5/1). After one hour of stirring (or when the aldehyde spot disappeared), the reaction mixture was quenched by pouring into a vigorously stirring $5 \mathrm{~mL}$ of cold sat. aq. $\mathrm{NaHCO}_{3}$ solution. The resulted slurry was stirred for $15 \mathrm{~min}$ and filtered through Celite. The layers were separated, and the aqueous layer was extracted with $10 \mathrm{~mL}$ of $\mathrm{CH}_{2} \mathrm{Cl}_{2}$. The organic phases were combined, washed with $2 \mathrm{~mL}$ of brine and dried over sodium sulfate. After filtration, the filtrate was concentrated. The crude product was purified by column chromatography (silica gel, hexane/EtOAc, 5/1), and $78 \mathrm{mg}(0.24 \mathrm{mmol}, 73 \%)$ of 28a was obtained as a clear viscous oil.

Data for 28a:

${ }^{1} \mathrm{H}$ NMR: $\quad\left(500 \mathrm{MHz}, \mathrm{CDCl}_{3}\right)$

syn-28a

7.37-7.26 (m, $5 \mathrm{H}$, aromatic); 5.17 (dt, $J=8.5,3.2,1 \mathrm{H}, \mathrm{HC}(5))$; 3.75-3.65 (ABX,

$\left.2 \mathrm{H}, \mathrm{H}_{2} \mathrm{C}(1)\right) ; 3.50$ (d, $\left.J=2.9,1 \mathrm{H}, \mathrm{HO}\right) ; 2.98-2.84\left(\mathrm{ABX}, 2 \mathrm{H}, \mathrm{H}_{2} \mathrm{C}(4)\right) ; 2.78$ (sext, $J=7.1,1 \mathrm{H}, \mathrm{HC}(2)) ; 1.03$ (d, $J=7.1,3 \mathrm{H}, \mathrm{H}_{3} \mathrm{C}(6)$ ); 0.87 (s, 9H, C(9)); 0.04 $(\mathrm{d}, J=1.7,6 \mathrm{H}, \mathrm{C}(7))$

anti-28a

7.37-7.26 (m, $5 \mathrm{H}$, aromatic); 5.14 (dt, $J=9.3,3.0,1 \mathrm{H}, \mathrm{HC}(5))$ ) 3.77-3.65 (ABX, $\left.2 \mathrm{H}, \mathrm{H}_{2} \mathrm{C}(1)\right) ; 3.54$ (d, $\left.J=3.0,1 \mathrm{H}, \mathrm{HO}\right) ; 2.98-2.84\left(\mathrm{ABX}, 2 \mathrm{H}, \mathrm{H}_{2} \mathrm{C}(4)\right) ; 2.79$ (sext, $J=7.1,1 \mathrm{H}, \mathrm{HC}(2)) ; 1.04$ (d, $\left.J=7.1,3 \mathrm{H}, \mathrm{H}_{3} \mathrm{C}(6)\right)$; 0.88 (s, 9H, C(9)); 0.04 $(\mathrm{d}, J=1.7,6 \mathrm{H})$

TLC: $\quad$ syn-28a $\mathrm{R}_{\mathrm{f}} 0.10$ (hexane/ether, 4/1, anisaldehyde reagent) anti-28a $\mathrm{R}_{\mathrm{f}} 0.15$ (hexane/ether, 4/1, anisaldehyde reagent)

SFC: $\quad(O D$ column, $175 \mathrm{bar}, 2.5 \mathrm{~mL} / \mathrm{min}, 1.7 \% \mathrm{MeOH}$ ) $t_{R} \operatorname{syn}$-28a $5.414 \min (91.3 \%) ; t_{R}$ anti-28a $5.989 \min (8.7 \%)$ 
General Procedure III: Aldol Addition of in-situ-generated Trichlorosilyl Enolates: $(1 S, 4 R)$-5-tert-Butyldimethylsilyloxy-4-methyl-1-hydroxy-1-phenyl-3-pentanone (syn-28a)

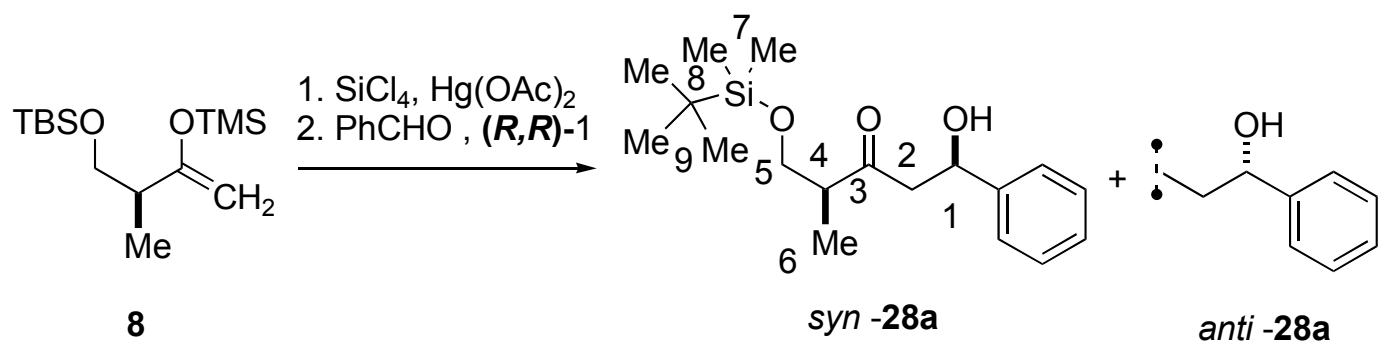

To a suspension of mercury(II) acetate $(3.2 \mathrm{mg}, 0.01 \mathrm{mmol}, 0.01$ equiv) in $1 \mathrm{~mL}$ of methylene chloride was added silicon tetrachloride $(0.23 \mathrm{~mL}, 2.0 \mathrm{mmol}, 2.0$ equiv) and the trimethylsilyl enol ether $8(288 \mathrm{mg}, 1.00 \mathrm{mmol})$. The reaction mixture was stirred at $\mathrm{rt}$ for 30 min. The excess silicon tetrachloride and the solvent were removed under vacuum. To the residue was added solution of the chiral phosphoramide $(R, R)-\mathbf{1}(36.9 \mathrm{mg}, 0.10 \mathrm{mmol}, 0.10$ equiv) in $2 \mathrm{~mL}$ of methylene chloride via a cannula. The reaction mixture was cooled to $-78{ }^{\circ} \mathrm{C}$ before the addition of benzaldehyde (102 $\mu \mathrm{L}, 1.00 \mathrm{mmol}, 1$ equiv). The reaction mixture was stirred at $-78^{\circ} \mathrm{C}$ for $2.5 \mathrm{~h}$, and then was quenched by pouring into a vigorously stirring $5 \mathrm{~mL}$ of cold saturated aqueous sodium bicarbonate. The resulting slurry was stirred for $1 \mathrm{~h}$ and was filtered through Celite. The layers were separated and the aqueous layer was extracted with 20 $\mathrm{mL}$ of methylene chloride. The combined organic extracts were washed with $5 \mathrm{~mL}$ of brine, dried over sodium sulfate and concentrated. The crude product was chromatographed (silica gel, hexane/ether, 5/1) to give $\mathbf{2 8 a}$ (268 $\mathrm{mg}, 0.83 \mathrm{mmol}, 83 \%)$ as viscous colorless oil.

Data for syn-28a:

${ }^{1} \mathrm{H}$ NMR: $\quad\left(500 \mathrm{MHz}, \mathrm{CDCl}_{3}\right)$

$7.23-7.47$ (m, 5H, aromatic); $5.17(\mathrm{dt}, J=8.8,3.2,1 \mathrm{H}, \mathrm{HC}(1)) ; 3.65-3.76$ $\left(\mathrm{ABX}, 2 \mathrm{H}, \mathrm{C}(5) H_{2}\right) ; 3.49(\mathrm{~d}, J=2.9,1 \mathrm{H}, \mathrm{OH}) ; 2.86-2.96\left(\mathrm{ABX}, 2 \mathrm{H}, \mathrm{C}(2) H_{2}\right)$; $2.78(\mathrm{~m}, 1 \mathrm{H}, \mathrm{C}(4) H) ; 1.03\left(\mathrm{~d}, J=6.9,3 \mathrm{H}, \mathrm{C}(6) H_{3}\right) ; 0.87\left(\mathrm{~s}, 9 \mathrm{H},\left(\mathrm{C}(9) H_{3}\right)_{3}\right) ; 0.04$ $\left(\mathrm{d}, J=2,6 \mathrm{H}, \mathrm{C}(7) H_{3}\right)$

${ }^{13} \mathrm{C} \mathrm{NMR}: \quad\left(125 \mathrm{MHz}, \mathrm{CDCl}_{3}\right)$

$214.7(\mathrm{C}(3)) ; 142.9,128.5,127.5,125.6$ (aromatic); $69.7(\mathrm{C}(1)) ; 65.6(\mathrm{C}(5)) ; 51.4$ $(\mathrm{C}(2)) ; 49.0(\mathrm{C}(4)) ; 25.8(\mathrm{C}(9)) ; 18.2(\mathrm{C}(8)) ; 12.6(\mathrm{C}(6)) ;-5.6(\mathrm{C}(7))$ 
IR: $\quad$ (neat)

3467 (m, br); 3064 (w); 3031 (w); 2954 (s); 2931 (s); 2885 (m); 2858 (m); 1708 (m); $1463(\mathrm{~m}) ; 1388(\mathrm{~m}) ; 1256(\mathrm{~m}) ; 1100(\mathrm{~s}) ; 838$ (s)

MS: $\quad(\mathrm{FI})$

$322\left(2, \mathrm{M}^{+}\right) ; 267$ (6); 266 (22); 266 (2); 265 (100); 247 (7); 159 (8); 106 (3); 95

Opt. rot. : $\quad+69.1\left(\mathrm{CHCl}_{3}, \mathrm{c}=1.0\right)$

TLC: $\quad R_{f}=0.19\left(\right.$ hexane/EtOAc, $\left.5 / 1, \mathrm{SiO}_{2}\right)$

SFC: (Chiralpak OD column, $150 \mathrm{bar}, 3.0 \mathrm{~mL} / \mathrm{min}, 1.4 \% \mathrm{MeOH}$ )

$t_{R}(2 S, 5 R)$-syn-28a $4.74 \min (95 \%) ;(2 S, 5 S)$-anti-28a $5.21 \min (5 \%)$

Analysis: $\quad$ Calc for $\mathrm{C}_{18} \mathrm{H}_{30} \mathrm{O}_{3} \mathrm{Si}(322.51)$

Calc. $\quad$ C $67.03 \%$; $9.38 \%$

Found: $\quad$ C $66.94 \%$; H $9.42 \%$

\section{(1S,4S)-5-tert-Butyldimethylsilyloxy-4-methyl-1-hydroxy-1-phenyl-3-pentanone (anti-28a)}

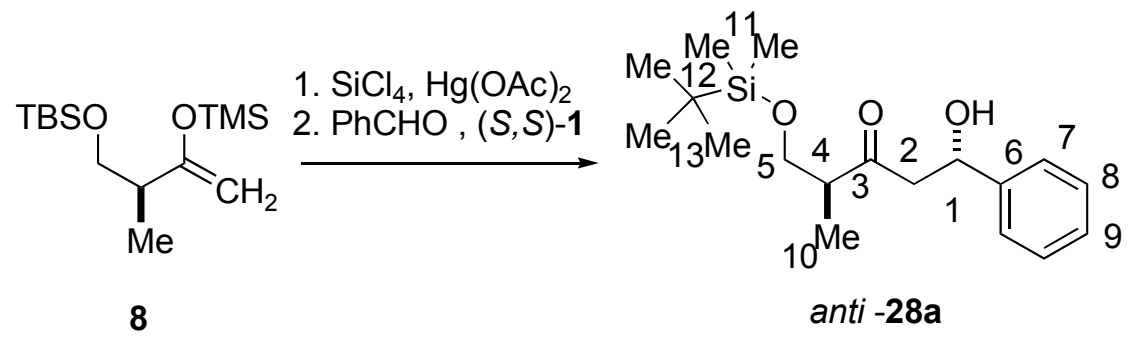

Following General Procedure III using 8 (288 mg, $1.00 \mathrm{mmol}), \mathrm{SiCl}_{4}(0.23 \mathrm{~mL}, 2.00$ mmol), $\mathrm{Hg}(\mathrm{OAc})_{2}(3.2 \mathrm{mg}, 0.01 \mathrm{mmol})$, benzaldehyde $(102 \mu \mathrm{L}, 1.00 \mathrm{mmol}),(S, S)-1$ (37 mg, $0.10 \mathrm{mmol})$ in $2 \mathrm{~mL}$ of $\mathrm{CH}_{2} \mathrm{Cl}_{2}, 121 \mathrm{mg}(0.38 \mathrm{mmol}, 75 \%)$ of anti-28a was obtained as colorless viscous oil after column chromatography (silica gel, hexane/EtOAc, 5/1).

Data for anti-28a:

글 $\quad\left(500 \mathrm{MHz}, \mathrm{CDCl}_{3}\right)$

$7.27-7.38(\mathrm{~m}, 5 \mathrm{H}$, aromatic); $5.14(\mathrm{dt}, J=9.6,2.8,1 \mathrm{H}, \mathrm{HC}(1)) ; 3.67-3.77$ $\left(\mathrm{ABX}, 2 \mathrm{H}, \mathrm{H}_{2} \mathrm{C}(5)\right) ; 3.50(\mathrm{~d}, J=3.0,1 \mathrm{H}, \mathrm{OH}) ; 2.84-2.97\left(\mathrm{ABX}, 2 \mathrm{H}, \mathrm{COC}(2) H_{2}\right)$; 
$2.78(\mathrm{~m}, 1 \mathrm{H}, \mathrm{COC}(4) H) ; 1.05\left(\mathrm{~d}, J=7.1,3 \mathrm{H}, \mathrm{CHC}(10) H_{3}\right) ; 0.88(\mathrm{~s}, 9 \mathrm{H}$, $\left.\left(\mathrm{C}(13) \mathrm{H}_{3}\right)_{3} \mathrm{CSi}\right) ; 0.05\left(\mathrm{~d}, J=2,6 \mathrm{H}, \mathrm{SiC}(11) H_{3}\right)$

${ }^{13} \mathrm{C} \mathrm{NMR}: \quad\left(125 \mathrm{MHz}, \mathrm{CDCl}_{3}\right)$

214.7 (C(3));142.9 (C(6)), 128.5 (C(8)), 127.5 (C(9)), 125.6 (C(7)); 70.0 (C(1)); $65.4(\mathrm{C}(5)) ; 51.1(\mathrm{C}(2)) ; 48.2(\mathrm{C}(4)) ; 25.8$ (C(13)); 18.2 (C(12)); 12.6 (C(10)); $5.6(\mathrm{C}(11))$

IR: (neat)

3467 (m, br); 3064 (w); 3032 (w); 2955 (s); 2931 (s); 2885 (m); 2859 (m); 1708 (s); $1463(\mathrm{~m}) ; 1388(\mathrm{~m}) ; 1256(\mathrm{~m}) ; 1100(\mathrm{~s})$

MS: $\quad(\mathrm{FI})$

$322\left(2, \mathrm{M}^{+}\right) ; 267$ (6); $266(21) ; 265$ (100); 247 (16); 159 (7); 106 (3); 95 (2)

Opt. rot. : $\quad-20.4\left(\mathrm{CHCl}_{3}\right.$, c=0.5)

TLC: $\quad R_{f}=0.17$ (hexane/EtOAc, $5 / 1, \mathrm{SiO}_{2}$ ) [p-anisaldehyde]

SFC: $\quad$ (Chiralpak OD column, $150 \mathrm{bar}, 3.0 \mathrm{~mL} / \mathrm{min}, 1.4 \% \mathrm{MeOH}$ )

$t_{R}(2 S, 5 R)$-syn-28a $4.81 \min (12 \%) ;(2 S, 5 S)$-anti-28a $5.16 \min (88 \%)$

Analysis: $\quad$ Calc for $\mathrm{C}_{18} \mathrm{H}_{30} \mathrm{O}_{3} \mathrm{Si}(322.51)$

Calc. $\quad$ C $67.03 \%$; $9.38 \%$

Found: $\quad$ C $66.81 \%$; $\quad$ H $9.20 \%$

(2S,5R)-1-tert-Butyldimethylsilyloxy-2-methyl-5-hydroxy-5-(1-naphthyl)-3-pentanone (syn28b)

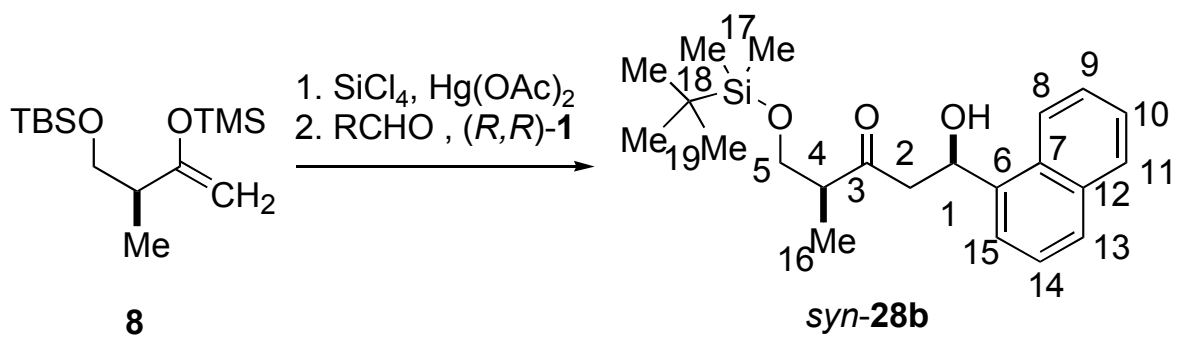

Following General Procedure III using 8 (288 mg, $1.00 \mathrm{mmol}), \mathrm{SiCl}_{4}(0.23 \mathrm{~mL}, 2.00$ $\mathrm{mmol}), \mathrm{Hg}(\mathrm{OAc})_{2}(3.2 \mathrm{mg}, 0.01 \mathrm{mmol})$, naphthaldehyde (156 mg, $\left.1.00 \mathrm{mmol}\right),(R, R)-1$ (37 mg, $0.10 \mathrm{mmol})$ in $2 \mathrm{~mL}$ of $\mathrm{CH}_{2} \mathrm{Cl}_{2}, 316 \mathrm{mg}(0.85 \mathrm{mmol}, 85 \%)$ of syn-28b was obtained as colorless 
viscous oil after column chromatography (silica gel, hexane/EtOAc, 5/1).

Data for syn-28b:

${ }^{1} \mathrm{H}$ NMR: $\quad\left(500 \mathrm{MHz}, \mathrm{CDCl}_{3}\right)$

$7.47-8.02$ (7H, aromatic); $5.97(\mathrm{dt}, J=9.5,2.5,1 \mathrm{H},(\mathrm{C}(1) H \mathrm{OH}) ; 3.68-3.80$ $\left(\mathrm{ABX}, 2 \mathrm{H}, \mathrm{TBSOC}(5) H_{2}\right) ; 3.58(\mathrm{~d}, J=2.9,1 \mathrm{H}, \mathrm{OH}) ; 2.98-3.13(\mathrm{ABX}, 2 \mathrm{H}$, $\left.\mathrm{COC}(2) H_{2}\right) ; 2.79(\mathrm{~m}, 1 \mathrm{H}, \mathrm{COC}(4) H) ; 1.06\left(\mathrm{~d}, J=7.1,3 \mathrm{H}, \mathrm{CHC}(16) H_{3}\right) ; 0.86$ (s, 9H, $\left.\left(\mathrm{C}(19) H_{3}\right)_{3} \mathrm{CSi}\right) ; 0.04\left(\mathrm{~d}, J=3.9,6 \mathrm{H}, \mathrm{SiC}(17) H_{3}\right)$

${ }^{13} \mathrm{C}$ NMR: $\quad\left(125 \mathrm{MHz}, \mathrm{CDCl}_{3}\right)$

$214.5(\mathrm{C}(3)) ; 138.4(\mathrm{C}(6)), 133.7(\mathrm{C}(12)), 129.9(\mathrm{C}(7)), 129.0(\mathrm{C}(11)) ; 127.9$ (C(13)); 126.2 (C(9)); 125.6 (C(10)); 125.5 (C(14)); 123.0 (C(15)); 122.8 (C(8)); $66.6(\mathrm{C}(1)) ; 65.5(\mathrm{C}(5)) ; 50.5(\mathrm{C}(2)) ; 49.2(\mathrm{C}(4)) ; 25.8(\mathrm{C}(19)) ; 18.2(\mathrm{C}(18)) ; 12.6$ $(\mathrm{C}(16)) ;-5.6(\mathrm{C}(17))$

IR: (neat)

3424 (m, br); 3053 (w); 2954 (s); 2931 (s); 2858 (m); 1708 (s); 1463 (m); 1389 (m); 1361 (m); 1257 (m); 1094 (s); 1030 (m)

MS: $\quad(\mathrm{FI})$

373 (34); $372\left(100, \mathrm{M}^{+}\right) ; 315$ (20); 267 (13); 267 (13); 267 (15); 267 (15); 240 (12); 159 (37); $156(58)$

Opt. rot. : $\quad+95.6\left(\mathrm{CHCl}_{3}, \mathrm{c}=1.0\right)$

TLC: $\quad R_{f}=0.18$ (hexane/EtOAc, $\left.5 / 1, \mathrm{SiO}_{2}\right)$ [ $p$-anisaldehyde]

SFC: $\quad$ (Chiralpak OD column, $150 \mathrm{bar}, 3.0 \mathrm{~mL} / \mathrm{min}, 12.5 \% \mathrm{MeOH}$ ):

$t_{R}(2 S, 5 S)$-anti-28b $3.23 \min (6 \%)$; (2S, 5R)-syn-28b $4.12 \min (94 \%)$,

Analysis: $\quad$ Calc for $\mathrm{C}_{22} \mathrm{H}_{32} \mathrm{O}_{3} \mathrm{Si}$ (372.57)

Calc. $\quad \mathrm{C} 70.92 \%$; $\mathrm{H} 8.66 \%$

Found: $\quad$ C $70.74 \%$; $\quad$ H $8.60 \%$ 
(2S,5S)-1-tert-Butyldimethylsilyloxy-2-methyl-5-hydroxy-5-(1-naphthyl)-3-pentanone (anti28b)

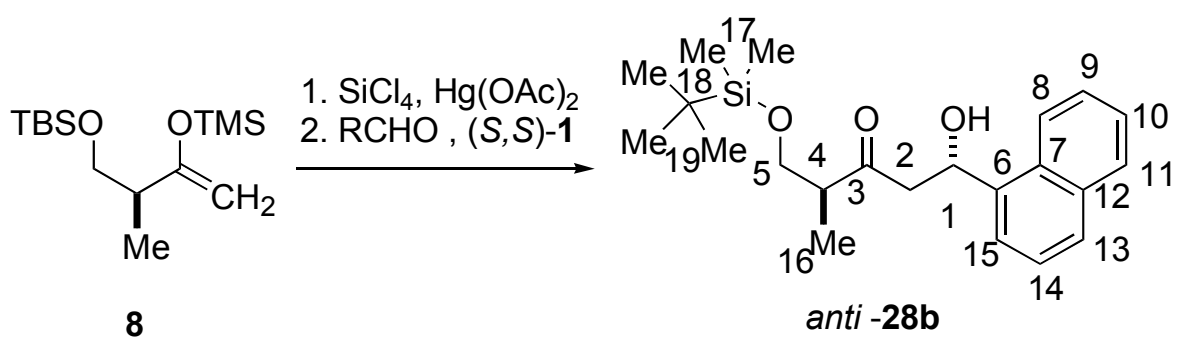

Following General Procedure III using 8 (288 mg, $1.00 \mathrm{mmol}), \mathrm{SiCl}_{4}(0.23 \mathrm{~mL}, 2.00 \mathrm{mmol})$, $\mathrm{Hg}(\mathrm{OAc})_{2}(3.2 \mathrm{mg}, 0.01 \mathrm{mmol})$, naphthaldehyde (156 mg, $\left.1.00 \mathrm{mmol}\right),(S, S)-1$ (37 mg, 0.10 $\mathrm{mmol})$ in $2 \mathrm{~mL}$ of $\mathrm{CH}_{2} \mathrm{Cl}_{2}, 308 \mathrm{mg}(0.85 \mathrm{mmol}, 85 \%)$ of anti-28b was obtained as colorless viscous oil after column chromatography (silica gel, hexane/EtOAc, 5/1).

Data for anti-28b:

${ }^{1} \mathrm{H}$ NMR: $\quad\left(500 \mathrm{MHz}, \mathrm{CDCl}_{3}\right)$

$7.47-8.02(7 \mathrm{H}$, aromatic); $5.98(\mathrm{~m}, J=9.5,1 \mathrm{H}, \mathrm{CHOH}) ; 3.69-3.79$ (ABX, $\left.2 \mathrm{H}, \mathrm{TBSOCH}_{2}\right) ; 3.68(\mathrm{~d}, \mathrm{~J}=2.8,1 \mathrm{H}, \mathrm{OH}) ; 3.01-3.09\left(\mathrm{ABX}, 2 \mathrm{H}, \mathrm{COCH}_{2}\right) ; 2.82$ $(\mathrm{m}, 1 \mathrm{H}, \mathrm{COCH}) ; 1.07\left(\mathrm{~d}, J=7.1,3 \mathrm{H}, \mathrm{CHCH}_{3}\right) ; 0.88\left(\mathrm{~s}, 9 \mathrm{H},\left(\mathrm{CH}_{3}\right)_{3} \mathrm{CSi}\right) ; 0.05(\mathrm{~d}, J$ $\left.=3.9,6 \mathrm{H}, \mathrm{SiCH}_{3}\right)$

${ }^{13} \mathrm{C} \mathrm{NMR}: \quad\left(125 \mathrm{MHz}, \mathrm{CDCl}_{3}\right)$

214.5 (C(3)); 138.4 (C(6)), 133.8 (C(12)), 129.9 (C(7)), 129.0 (C(11)); 128.0 (C(13)); 126.1 (C(9)); 125.6 (C(10)); 125.5 (C(14)); 123.0 (C(15)); 122.8 (C(8)); 66.9 (C(1)); $65.5(\mathrm{C}(5)) ; 50.2(\mathrm{C}(2)) ; 49.3(\mathrm{C}(4)) ; 25.8$ (C(19)); 18.2 (C(18)); 12.7 $(\mathrm{C}(16)) ;-5.6(\mathrm{C}(17))$

IR: (neat) 3479 (m, br); 3053 (w); 2954 (s); 2930 (s); 2885 (m); 2858 (m); 1706 (s); 1463 (m); 1390 (m); 1361 (m); 1256 (m); 1098 (s)

MS: $\quad(\mathrm{FI})$

373 (29); 372 (100, M+); 315 (12); 267 (13); 267 (13); 267 (11); 159 (32); 156 (44)

Opt. rot. : $\quad-63.5\left(\mathrm{CHCl}_{3}, \mathrm{c}=1.0\right)$ 
TLC: $\quad R_{f}=0.19$ (hexane/EtOAc, $5 / 1, \mathrm{SiO}_{2}$ ) [p-anisaldehyde]

SFC: (Chiralpak OD column, 150 bar, $3.0 \mathrm{~mL} / \mathrm{min}, 12.5 \% \mathrm{MeOH}$ ):

$t_{R}(2 S, 5 S)$-anti-28b $3.15 \min (89 \%) ;(2 S, 5 R)$-syn-28b $4.11 \min (11 \%)$

Analysis: $\quad$ Calc for $\mathrm{C}_{22} \mathrm{H}_{32} \mathrm{O}_{3} \mathrm{Si}(372.57)$

Calc. $\quad \mathrm{C} 70.92 \% ; \quad \mathrm{H} 8.66 \%$

Found: $\quad$ C $70.63 \% ; \quad$ H $8.58 \%$

$(2 S, 5 R, 6 E)$-1-tert-Butyldimethylsilyloxy-2-methyl-5-hydroxy-7-phenyl-6-hepten-3-one (syn28c)

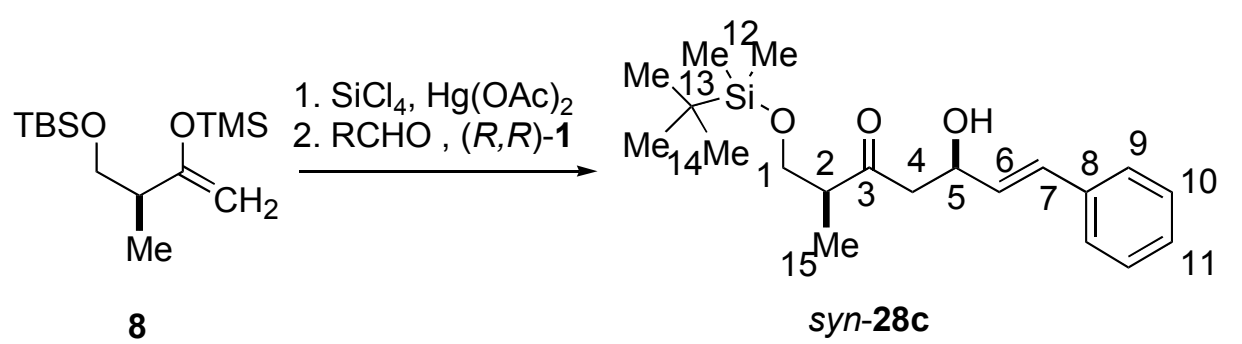

Following General Procedure III using 8 (288 mg, $1.00 \mathrm{mmol}), \mathrm{SiCl}_{4}(0.23 \mathrm{~mL}, 2.00$ $\mathrm{mmol}), \mathrm{Hg}(\mathrm{OAc})_{2}(3.2 \mathrm{mg}, 0.01 \mathrm{mmol})$, cinnamaldehyde (132 mg, $\left.1.00 \mathrm{mmol}\right),(R, R)-1(37 \mathrm{mg}$, $0.10 \mathrm{mmol})$ in $2 \mathrm{~mL}$ of $\mathrm{CH}_{2} \mathrm{Cl}_{2}, 281 \mathrm{mg}(0.81 \mathrm{mmol}, 81 \%)$ of syn-28c was obtained as colorless viscous oil after column chromatography (silica gel, hexane/EtOAc, 5/1).

Data for syn-28c:

1H NMR: $\quad\left(500 \mathrm{MHz}, \mathrm{CDCl}_{3}\right)$

7.37 (d, $J=8.6,1 \mathrm{H}$, aromatic); 7.37 (d, $J=5.6,1 \mathrm{H}$, aromatic); 7.30 (t, $J=7.7$, $2 \mathrm{H}$, aromatic); $7.23(\mathrm{t}, J=7.2,1 \mathrm{H}$, aromatic); $6.65(\mathrm{~d}, J=16.2,1 \mathrm{H}, \mathrm{CH}) ; 6.21$ $(\mathrm{dd}, J=16.0,6.0,1 \mathrm{H}, \mathrm{CH}) ; 4.77(\mathrm{~m}, 1 \mathrm{H}, \mathrm{CHOH}) ; 3.66-3.77(\mathrm{ABX}$, 2H,TBSOCH$\left.H_{2}\right) ; 3.31(\mathrm{~d}, J=3.4,1 \mathrm{H}, \mathrm{OH}) ; 2.75-2.90\left(\mathrm{ABX}, 2 \mathrm{H}, \mathrm{COCH}_{2}\right) ; 2.82$ $(\mathrm{m}, 1 \mathrm{H}, \mathrm{COCH}) ; 1.06\left(\mathrm{~d}, J=7.1,3 \mathrm{H}, \mathrm{CHCH}_{3}\right) ; 0.88\left(\mathrm{~s}, 9 \mathrm{H},\left(\mathrm{CH}_{3}\right)_{3} \mathrm{CSi}\right) ; 0.05(\mathrm{~d}, J$ $=5.7,6 \mathrm{H}, \mathrm{SiCH}_{3}$ )

${ }^{13} \mathrm{C} \mathrm{NMR}: \quad\left(125 \mathrm{MHz}, \mathrm{CDCl}_{3}\right)$

$214.4(\mathrm{C}(3)) ; 136.6(\mathrm{C}(8)), 130.2(\mathrm{C}(11)), 130.0(\mathrm{C}(7)), 128.5(\mathrm{C}(10)) ; 127.6$ (C(6)); 126.4 (C(9)); 68.2 (C(1)); $65.5(\mathrm{C}(5)) ; 49.3$ (C(2)); 49.0 (C(4)); 25.8 $(\mathrm{C}(14)) ; 18.1(\mathrm{C}(13)) ; 12.6(\mathrm{C}(15)) ;-5.6,-5.6(\mathrm{C}(12))$

IR: (neat) 
3438 (m, br); 3027 (w); 2954 (s); 2930 (s); 2858 (m); 1708 (s); 1470 (m); 1388 (m); $1256(\mathrm{~m}) ; 1099(\mathrm{~s})$

MS: $\quad(\mathrm{FI})$

349 (29); $348\left(100, \mathrm{M}^{+}\right) ; 291(8) ; 216(6) ; 159(32) ; 132$ (42)

Opt. rot. : $\quad+32.2\left(\mathrm{CHCl}_{3}, \mathrm{c}=1.0\right)$

TLC: $\quad R_{f}=0.12\left(\right.$ hexane/EtOAc, $\left.5 / 1, \mathrm{SiO}_{2}\right)$ [p-anisaldehyde]

SFC: $\quad$ (Chiralpak OD column, $150 \mathrm{bar}, 3.0 \mathrm{~mL} / \mathrm{min}, 7.5 \% \mathrm{MeOH}$ ):

$t_{R}(2 S, 5 R)$-syn-28c $2.74 \min (89 \%) ;(2 S, 5 S)$-anti-28c $3.26 \min (11 \%)$

Analysis: $\quad$ Calc for $\mathrm{C}_{20} \mathrm{H}_{32} \mathrm{O}_{3} \mathrm{Si}(348.55)$

Calc. $\quad$ C $68.92 \%$; $9.38 \%$

Found: $\quad$ C $68.59 \%$; H $9.02 \%$

(2S,5S,6E)-1-tert-Butyldimethylsilyloxy-2-methyl-5-hydroxy-7-phenyl-6-hepten-3-one (anti28c)

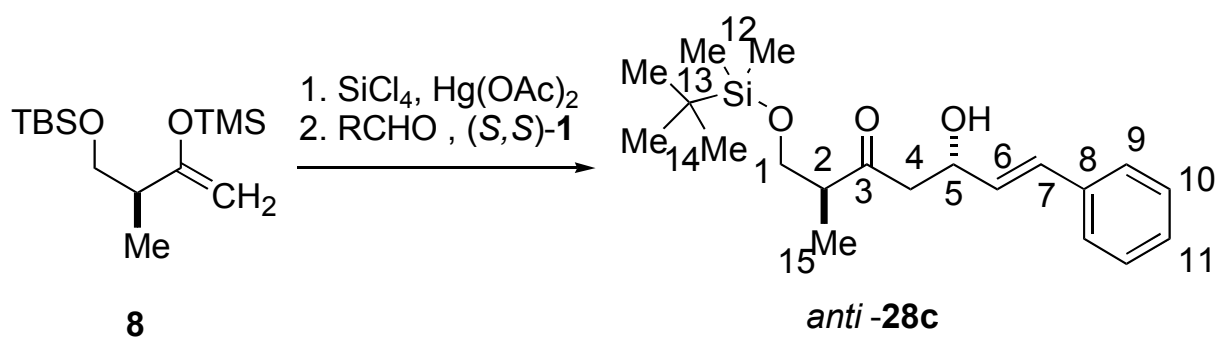

Following General Procedure III using 8 (288 mg, $1.00 \mathrm{mmol}), \mathrm{SiCl}_{4}(0.23 \mathrm{~mL}, 2.00$ $\mathrm{mmol}), \mathrm{Hg}(\mathrm{OAc})_{2}(3.2 \mathrm{mg}, 0.01 \mathrm{mmol})$, cinnamaldehyde (132 mg, $\left.1.00 \mathrm{mmol}\right),(S, S)-1$ (37 mg, $0.10 \mathrm{mmol})$ in $2 \mathrm{~mL}$ of $\mathrm{CH}_{2} \mathrm{Cl}_{2}, 286 \mathrm{mg}(0.83 \mathrm{mmol}, 83 \%)$ of anti-28c was obtained as colorless viscous oil after column chromatography (silica gel, hexane/EtOAc, 5/1).

Data for anti-28c:

${ }^{1} \mathrm{H} \mathrm{NMR}: \quad\left(500 \mathrm{MHz}, \mathrm{CDCl}_{3}\right)$

$7.37(\mathrm{~d}, J=8.6,1.0,1 \mathrm{H}$, aromatic); $7.37(\mathrm{~d}, J=7.7,1.0,1 \mathrm{H}$, aromatic); $7.31(\mathrm{t}, J$ $=7.7,2 \mathrm{H}$, aromatic); $7.23(\mathrm{t}, J=7.2,1 \mathrm{H}$, aromatic); $6.67(\mathrm{dd}, J=16.1,1.4,1 \mathrm{H}$, $\mathrm{CH}) ; 6.22(\mathrm{dd}, J=15.9,6.0,1 \mathrm{H}, \mathrm{CH}) ; 4.75(\mathrm{~m}, 1 \mathrm{H}, \mathrm{CHOH}) ; 3.67$ - $3.78(\mathrm{ABX}$, $\left.2 \mathrm{H}, \mathrm{TBSOCH}_{2}\right) ; 3.34(\mathrm{~d}, \mathrm{~J}=3.4,1 \mathrm{H}, \mathrm{OH}) ; 2.77-2.92\left(\mathrm{ABX}, 2 \mathrm{H}, \mathrm{COCH}_{2}\right) ; 2.84$ 
$(\mathrm{m}, 1 \mathrm{H}, \mathrm{COCH}) ; 1.07$ (d, $\left.J=7.1,3 \mathrm{H}, \mathrm{CHCH}_{3}\right) ; 0.88\left(\mathrm{~s}, 9 \mathrm{H},\left(\mathrm{CH}_{3}\right)_{3} \mathrm{CSi}\right) ; 0.05$ (d, $J$ $\left.=3.7,6 \mathrm{H}, \mathrm{SiCH}_{3}\right)$

${ }^{13} \mathrm{C} \mathrm{NMR}: \quad\left(125 \mathrm{MHz}, \mathrm{CDCl}_{3}\right)$

214.3 (C(3)); $136.7(\mathrm{C}(8)), 130.3$ (C(11)), 130.2 (C(7)), 128.5 (C(10)); 127.5 (C(6)); 126.5 (C(9)); 68.6 (C(1)); 65.4 (C(5)); 49.3 (C(2)); 48.9 (C(4)); 25.8 $(\mathrm{C}(14)) ; 18.2(\mathrm{C}(13)) ; 12.7$ (C(15)); -5.6 (C(12))

IR: $\quad$ (neat)

3446 (m, br); 3027 (w); 2954 (s); 2930 (s); 2858 (m); 1708 (s); 1470 (m); 1388 (m); $1256(\mathrm{~m}) ; 1101(\mathrm{~s})$

$\underline{\mathrm{MS}}: \quad(\mathrm{FI})$

348 (1, M+); 331 (4); 291 (6); 273 (9); 217 (3); 201 (7); 159 (100); 133 (35); 115 (37); 103 (20); 89 (10); 75 (56)

Opt. rot. : $\quad+6.36\left(\mathrm{CHCl}_{3}, \mathrm{c}=1.0\right)$

TLC: $\quad R_{f}=0.15$ (hexane/EtOAc, $\left.5 / 1, \mathrm{SiO}_{2}\right)[p$-anisaldehyde]

SFC: $\quad$ (Chiralpak OD column, $150 \mathrm{bar}, 3.0 \mathrm{~mL} / \mathrm{min}, 7.5 \% \mathrm{MeOH}$ ):

$t_{R}(2 S, 5 R)-s y n-28 c 2.55 \min (19 \%) ;(2 S, 5 S)$-anti-28c $2.96 \min (81 \%)$

Analysis: $\quad$ Calc for $\mathrm{C}_{20} \mathrm{H}_{32} \mathrm{O}_{3} \mathrm{Si}(348.55)$

Calc. $\quad$ C $68.92 \%$; $9.38 \%$

Found: $\quad$ C $68.56 \%$; H $9.14 \%$

(2S,5R,6E)-1-tert-Butyldimethylsilyloxy-2,6-dimethyl-5-hydroxy-7-phenyl-6-hepten-3-one (syn-28d)

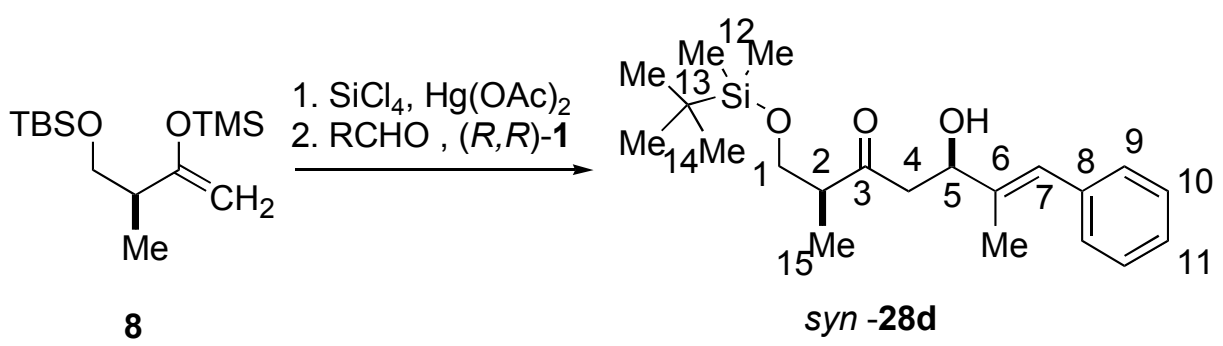

Following General Procedure III using 8 (288 mg, $1.00 \mathrm{mmol}), \mathrm{SiCl}_{4}(0.23 \mathrm{~mL}, 2.00$ 
mmol), $\mathrm{Hg}(\mathrm{OAc})_{2}(3.2 \mathrm{mg}, 0.01 \mathrm{mmol}), \alpha$-methylcinnamaldehyde (146 mg, $\left.1.00 \mathrm{mmol}\right),(R, R)-1$ (37 mg, $0.10 \mathrm{mmol}$ ) in $2 \mathrm{~mL}$ of $\mathrm{CH}_{2} \mathrm{Cl}_{2}, 303 \mathrm{mg}(0.84 \mathrm{mmol}, 84 \%$ ) of syn-28d was obtained as colorless viscous oil after column chromatography (silica gel, hexane/EtOAc, 5/1).

Data for syn-28d:

${ }^{1} \mathrm{H}$ NMR: $\quad\left(500 \mathrm{MHz}, \mathrm{CDCl}_{3}\right)$

$7.33(\mathrm{t}, J=7.7,2 \mathrm{H}$, aromatic); 7.27 (d, $J=5.4,2 \mathrm{H}$, aromatic); $7.21(\mathrm{t}, J=7.4,1 \mathrm{H}$, aromatic); $6.58(\mathrm{~s}, 1 \mathrm{H}, \mathrm{CH}) ; 4.64(\mathrm{dt}, J=9.5,2.61 \mathrm{H}, \mathrm{CHOH}) ; 3.67$ - $3.77(\mathrm{ABX}$, $\left.2 \mathrm{H}, \mathrm{TBSOCH}_{2}\right) ; 3.27(\mathrm{~d}, \mathrm{~J}=3.0,1 \mathrm{H}, \mathrm{OH}) ; 2.77-2.89$ (m, 3H, $\left.\mathrm{COCH}_{2}, \mathrm{COCH}\right)$; 1.88 (s, 3H, $\left.\mathrm{CH}_{3}\right) ; 1.06\left(\mathrm{~d}, J=7.1,3 \mathrm{H}, \mathrm{CHCH}_{3}\right) ; 0.88\left(\mathrm{~s}, 9 \mathrm{H},\left(\mathrm{CH}_{3}\right)_{3} \mathrm{CSi}\right) ; 0.06$ (d, $\left.J=5.1,6 \mathrm{H}, \mathrm{SiCH}_{3}\right)$

${ }^{13} \mathrm{C} \mathrm{NMR}: \quad\left(125 \mathrm{MHz}, \mathrm{CDCl}_{3}\right)$

214.9 (C(3)); $138.4(\mathrm{C}(6))$; 137.5 (C(8)); 128.9 (C(10)); 128.0 (C(9)) 126.4 (C(11)), 125.5 (C(7)); 72.7 (C(5)); 65.6 (C(1)) 49.0 (C(2)); 48.2 (C(4)); 25.7 $(\mathrm{C}(14)) ; 18.1(\mathrm{C}(13)) ; 14.0(\mathrm{C}(16)) ; 13.0(\mathrm{C}(15)) ;-5.6,-5.7(\mathrm{C}(12))$

IR: $\quad$ (neat)

3446 (m, br); 3056(w); 3025 (w); 2954 (s); 2931 (s); 2885 (m); 2859 (m); 1708 (s); 1463 (m); 1388 (m); 1257 (m); 1100 (s); 1007 (m)

MS: $\quad(\mathrm{FI})$

364 (8); 364.0 (32); $362\left(100, \mathrm{M}^{+}\right) ; 362$ (3); 327 (3); 305 (6); 257 (3); 216 (3); 160 (3); 159 (29); $153(4) ; 147$ (5); $146(39)$

Opt. rot. : $\quad+36.3\left(\mathrm{CHCl}_{3}, \mathrm{c}=1.0\right)$

TLC: $\quad R_{f}=0.17$ (hexane/EtOAc, $5 / 1, \mathrm{SiO}_{2}$ ) [ $p$-anisaldehyde]

SFC: (Chiralpak OD column, $150 \mathrm{bar}, 3.0 \mathrm{~mL} / \mathrm{min}, 7.5 \% \mathrm{MeOH}$ ):

$t_{R}(2 S, 5 R)$-syn-28d $2.76 \min (91 \%) ;(2 S, 5 S)$-anti-28d $3.03 \min (9 \%)$

Analysis: $\quad$ Calc for $\mathrm{C}_{21} \mathrm{H}_{34} \mathrm{O}_{3} \mathrm{Si}(362.58)$

Calc. $\quad \mathrm{C} 69.56 \% ; \quad \mathrm{H} 9.45 \%$

Found: $\quad$ C $69.53 \%$; $\quad$ H $9.45 \%$ 
(2S,5S,6E)-1-tert-Butyldimethylsilyloxy-2,6-dimethyl-5-hydroxy-7-phenyl-6-hepten-3-one (anti-28d)

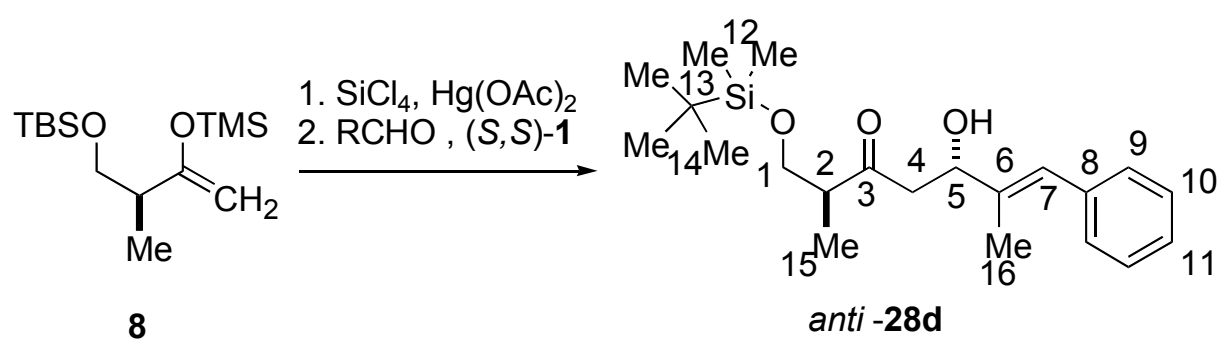

Following General Procedure III using 8 (288 mg, $1.00 \mathrm{mmol}), \mathrm{SiCl}_{4}(0.23 \mathrm{~mL}, 2.00$ $\mathrm{mmol}), \mathrm{Hg}(\mathrm{OAc})_{2}(3.2 \mathrm{mg}, 0.01 \mathrm{mmol}), \alpha$-methylcinnamaldehyde (146 mg, $\left.1.00 \mathrm{mmol}\right),(S, S)-\mathbf{1}$ (37 mg, $0.10 \mathrm{mmol})$ in $2 \mathrm{~mL}$ of $\mathrm{CH}_{2} \mathrm{Cl}_{2}, 285 \mathrm{mg}(0.80 \mathrm{mmol}, 80 \%)$ of anti-28d was obtained as colorless viscous oil after column chromatography (silica gel, hexane/EtOAc, 5/1).

Data for anti-28d:

${ }^{1} \mathrm{H} \mathrm{NMR}: \quad\left(500 \mathrm{MHz}, \mathrm{CDCl}_{3}\right)$

7.33 (t, $J=7.6,2 \mathrm{H}$, aromatic); 7.27 (d, $J=6.6,2 \mathrm{H}$, aromatic); $7.21(\mathrm{t}, J=7.2,1 \mathrm{H}$, aromatic); $6.58(\mathrm{~s}, 1 \mathrm{H}, \mathrm{CH}) ; 4.62(\mathrm{dt}, J=9.0,2.81 \mathrm{H}, \mathrm{CHOH}) ; 3.68$ - 3.78 (ABX, 2H,TBSOCH$\left.)_{2}\right) ; 3.30(\mathrm{~d}, J=2.9,1 \mathrm{H}, \mathrm{OH}) ; 2.78-2.88\left(\mathrm{~m}, 3 \mathrm{H}, \mathrm{COCH}_{2}, \mathrm{COCH}\right)$; $1.88\left(\mathrm{~d}, J=1.3,3 \mathrm{H}, \mathrm{CH}_{3}\right) ; 1.08\left(\mathrm{~d}, J=7.1,3 \mathrm{H}, \mathrm{CHCH}_{3}\right) ; 0.89\left(\mathrm{~s}, 9 \mathrm{H},\left(\mathrm{CH}_{3}\right)_{3} \mathrm{CSi}\right)$; $0.06\left(\mathrm{~d}, J=5.1,6 \mathrm{H}, \mathrm{SiCH}_{3}\right)$

${ }^{13} \mathrm{C} \mathrm{NMR}: \quad\left(125 \mathrm{MHz}, \mathrm{CDCl}_{3}\right)$

214.9 (C(3)); 138.4 (C(6)); 137.5 (C(8)); 128.9 (C(10)); 128.0 (C(9)) 126.4 (C(11)), 125.6 (C(7)); 73.1 (C(5)); 65.5 (C(1)) 49.3 (C(2)); 47.8 (C(4)); 25.8 $(\mathrm{C}(14)) ; 18.2(\mathrm{C}(13)) ; 13.9(\mathrm{C}(16)) ; 12.7(\mathrm{C}(15)) ;-5.6(\mathrm{C}(12))$

IR: (neat)

3468 (m, br); 3057(w); 3025 (w); 2955 (s); 2931 (s); 2885 (m); $2856(\mathrm{~m}) ; 1707$ (s); 1463 (m); 1388 (m); 1361 (m); 1256 (m); 1099 (s); 1007 (m)

MS: $\quad(\mathrm{FI})$

364 (7); 364 (29); $362\left(100, \mathrm{M}^{+}\right) ; 327$ (2); 305 (5); 160 (2); 159 (20); 153 (3); 147 (3); $146(26)$

Opt. rot. : $\quad+6.55\left(\mathrm{CHCl}_{3}, \mathrm{c}=1.0\right)$ 
TLC: $\quad R_{f}=0.19$ (hexane/EtOAc, $5 / 1, \mathrm{SiO}_{2}$ ) [p-anisaldehyde]

SFC: (Chiralpak OD column, $150 \mathrm{bar}, 3.0 \mathrm{~mL} / \mathrm{min}, 7.5 \% \mathrm{MeOH}$ ):

$t_{R}(2 S, 5 R)$-syn-28d $2.65 \min (13 \%) ;(2 S, 5 S)$-anti-28d $2.91 \min (87 \%)$

Analysis: $\quad$ Calc for $\mathrm{C}_{21} \mathrm{H}_{34} \mathrm{O}_{3} \mathrm{Si}(362.58)$

Calc. $\quad \mathrm{C} 69.56 \%$; $\mathrm{H} 9.45 \%$

Found: $\quad$ C $69.52 \%$; H $9.49 \%$

(2S,5R,6E)-1-tert-Butyldimethylsilyloxy-2-methyl-5-hydroxy-6-octen-3-one (syn-28e)

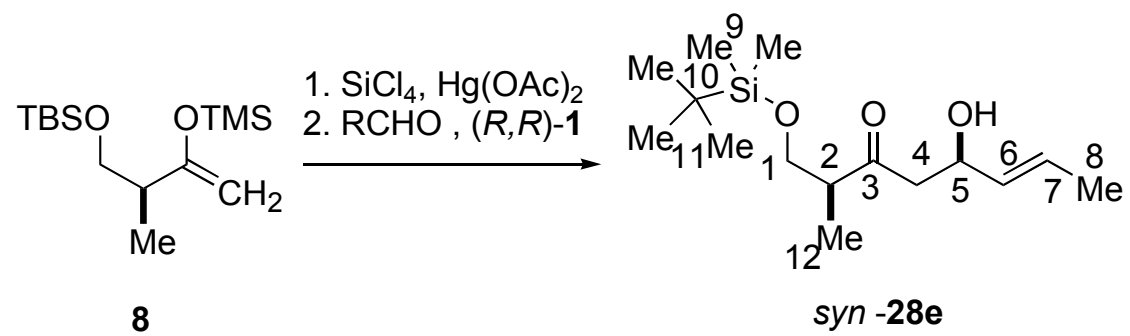

Following General Procedure III using $8(288 \mathrm{mg}, 1.00 \mathrm{mmol}), \mathrm{SiCl}_{4}(0.23 \mathrm{~mL}, 2.00$ $\mathrm{mmol}), \mathrm{Hg}(\mathrm{OAc})_{2}(3.2 \mathrm{mg}, 0.01 \mathrm{mmol})$, crotonaldehyde $(70 \mathrm{mg}, 1.00 \mathrm{mmol}),(R, R)-\mathbf{1}(37 \mathrm{mg}$, $0.10 \mathrm{mmol})$ in $2 \mathrm{~mL}$ of $\mathrm{CH}_{2} \mathrm{Cl}_{2}, 244 \mathrm{mg}(0.84 \mathrm{mmol}, 84 \%)$ of syn-28e was obtained as colorless viscous oil after column chromatography (silica gel, hexane/EtOAc, 5/1).

Data for syn-28e:

${ }^{1} \mathrm{H}$ NMR: $\quad\left(500 \mathrm{MHz}, \mathrm{CDCl}_{3}\right)$;

5.71 (dqd, $J=15.4,6.6,1.0,1 \mathrm{H}, \mathrm{CH}) ; 5.49$ (ddq, $J=15.5,6.6,1.4,1 \mathrm{H}, \mathrm{CH}) ; 4.52$ $(\mathrm{m}, 1 \mathrm{H}, \mathrm{CHOH}) ; 3.63$ - $3.74\left(\mathrm{ABX}, 2 \mathrm{H}, \mathrm{TBSOCH}_{2}\right) ; 3.13(\mathrm{~d}, J=3.4,1 \mathrm{H}, \mathrm{OH})$; $2.64-2.78\left(\mathrm{ABX}, 2 \mathrm{H}, \mathrm{COCH}_{2}\right) ; 2.78(\mathrm{~m}, 1 \mathrm{H}, \mathrm{COCH}) ; 1.69$ (dt, $J=6.6,0.8,3 \mathrm{H}$, $\left.\mathrm{CH}_{3}\right) ; 1.02\left(\mathrm{~d}, J=6.8,3 \mathrm{H}, \mathrm{CHCH}_{3}\right) ; 0.87$ (s, $\left.9 \mathrm{H},\left(\mathrm{CH}_{3}\right)_{3} \mathrm{CSi}\right) ; 0.03$ (d, $J=6.4,6 \mathrm{H}$, $\left.\mathrm{SiCH}_{3}\right)$

${ }^{13} \mathrm{C} \mathrm{NMR}: \quad\left(125 \mathrm{MHz}, \mathrm{CDCl}_{3}\right)$

214.8 (C(3)); $132.0(\mathrm{C}(6)) ; 126.9(\mathrm{C}(7)) ; 68.4(\mathrm{C}(5)) ; 65.6(\mathrm{C}(1)) ; 49.3(\mathrm{C}(2))$; $49.0(\mathrm{C}(4)) ; 25.8(\mathrm{C}(11)) ; 18.2(\mathrm{C}(10)) ; 17.7(\mathrm{C}(8)) 12.6(\mathrm{C}(12))$; -5.6, -5.6 (C(9))

IR: (neat)

3444 (m, br); 2956 (s); 2932 (s); 2886 (m); 2859 (m); 1708 (s); 1463 (m); 1388 
(m); $1362(\mathrm{w}) ; 1257(\mathrm{~m}) ; 1099(\mathrm{~s})$

$\underline{\mathrm{MS}}: \quad(\mathrm{FI})$

$286\left(2, \mathrm{M}^{+}\right) ; 231(5) ; 230(18) ; 229$ (100); $211(3) ; 159(5) ; 113$ (2)

Opt. rot. : $\quad+36.7\left(\mathrm{CHCl}_{3}, \mathrm{c}=1.0\right)$

TLC: $\quad R_{f}=0.12\left(\right.$ hexane/EtOAc, $\left.5 / 1, \mathrm{SiO}_{2}\right)$ [p-anisaldehyde]

SFC: $\quad$ (5-benzoyloxy-28e, Chiralpak OD column, $150 \mathrm{bar}, 3.0 \mathrm{~mL} / \mathrm{min}, 1.5 \% \mathrm{MeOH}$ ):

$t_{R}(2 S, 5 R)$-syn-5-benzoyloxy-28e $4.44 \min (83 \%)$; (2S, 5S)-anti-5-benzoyloxy-

28e $5.26 \min (17 \%)$

Analysis: $\quad$ Calc for $\mathrm{C}_{15} \mathrm{H}_{20} \mathrm{O}_{3} \mathrm{Si}$ (286.48)

Calc. C $62.89 \%$; $10.55 \%$

Found: $\quad$ C $62.69 \%$; H $10.67 \%$

\section{(2S,5S,6E)-1-tert-Butyldimethylsilyloxy-2-methyl-5-hydroxy-6-octen-3-one (anti-28e)}

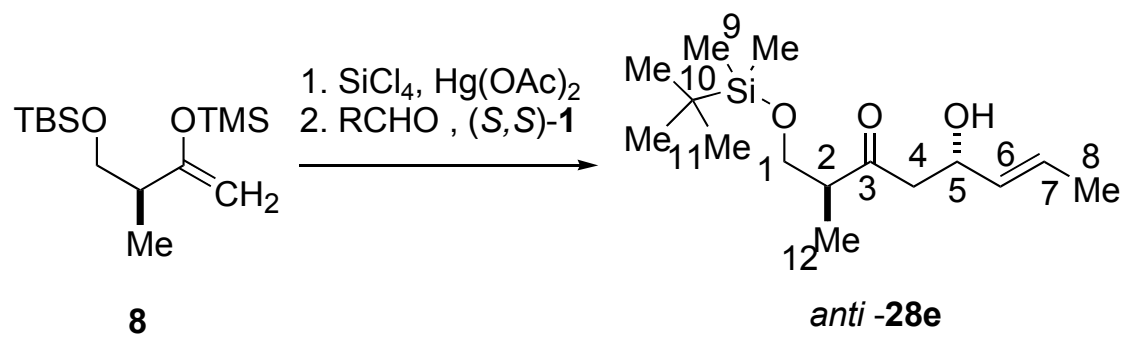

Following General Procedure III using 8 (288 mg, $1.00 \mathrm{mmol}), \mathrm{SiCl}_{4}(0.23 \mathrm{~mL}, 2.00$ $\mathrm{mmol}), \mathrm{Hg}(\mathrm{OAc})_{2}(3.2 \mathrm{mg}, 0.01 \mathrm{mmol})$, crotonaldehyde $(70 \mathrm{mg}, 1.00 \mathrm{mmol}),(S, S)-1$ (37 mg, $0.10 \mathrm{mmol})$ in $2 \mathrm{~mL}$ of $\mathrm{CH}_{2} \mathrm{Cl}_{2}, 238 \mathrm{mg}(0.83 \mathrm{mmol}, 83 \%)$ of anti-28e was obtained as colorless viscous oil after column chromatography (silica gel, hexane/EtOAc, 5/1).

Data for anti-28e:

${ }^{1} \mathrm{H}$ NMR: $\quad\left(500 \mathrm{MHz}, \mathrm{CDCl}_{3}\right)$;

$5.69(\mathrm{dqd}, J=15.1,6.6,1.0,1 \mathrm{H}, \mathrm{CH}) ; 5.48(\mathrm{ddq}, J=15.5,6.6,1.2,1 \mathrm{H}, \mathrm{CH}) ; 4.47$ (m, 1H, CHOH); 3.61 - $3.74\left(\mathrm{ABX}, 2 \mathrm{H}, \mathrm{TBSOCH}_{2}\right) ; 3.15(\mathrm{~d}, J=3.4,1 \mathrm{H}, \mathrm{OH})$; $2.62-2.77\left(\mathrm{~m}, 3 \mathrm{H}, \mathrm{COCH}_{2}, \mathrm{COCH}\right) ; 1.67\left(\mathrm{dd}, J=6.3,0.7,3 \mathrm{H}, \mathrm{CH}_{3}\right) ; 1.02$ (dd, $J$ $\left.=6.9,1.0,3 \mathrm{H}, \mathrm{CHCH}_{3}\right) ; 0.85\left(\mathrm{~d}, J=0.9,9 \mathrm{H},\left(\mathrm{CH}_{3}\right)_{3} \mathrm{CSi}\right) ; 0.02(\mathrm{~d}, J=3.2,6 \mathrm{H}$, $\left.\mathrm{SiCH}_{3}\right)$ 
${ }^{13} \mathrm{C} \mathrm{NMR}: \quad\left(101 \mathrm{MHz}, \mathrm{CDCl}_{3}\right)$

$219.6(\mathrm{C}(3)) ; 132.0(\mathrm{C}(6)) ; 126.8(\mathrm{C}(7)) ; 68.6(\mathrm{C}(5)) ; 65.3(\mathrm{C}(1)) ; 49.1$ (C(2));

48.9 (C(4)); 25.7 (C(11)); 18.1 (C(10)); $17.6(\mathrm{C}(8)) 12.6(\mathrm{C}(12)) ;-5.7(\mathrm{C}(9))$

IR: (neat)

3448 (m, br); 2956 (s); 2932 (s); 2886 (m); 2859 (m); 1708 (s); 1463 (m); 1388

(m); $1257(\mathrm{~m}) ; 1099(\mathrm{~s})$

$\underline{\mathrm{MS}}: \quad(\mathrm{FI})$

287 (4); 286 (3, M+); 231 (4); 230 (19); 229 (100); 159 (4); 113 (3); 113 (3); 111

(3); $110(3) ; 70(3)$

Opt. rot. : $\quad+15.6\left(\mathrm{CHCl}_{3}, \mathrm{c}=1.0\right)$

TLC: $\quad R_{f}=0.18$ (pentane/ether, $\left.3 / 1, \mathrm{SiO}_{2}\right)[p$-anisaldehyde]

SFC: $\quad$ (5-benzoyloxy-28e, Chiralpak OD column, $150 \mathrm{bar}, 3.0 \mathrm{~mL} / \mathrm{min}, 1.5 \% \mathrm{MeOH}$ ):

$t_{R}(2 S, 5 R)$-syn-5-benzoyloxy-28e $4.47 \min (26 \%)$; (2S, 5S)-anti-5-benzoyloxy-

28e $5.14 \min (74 \%)$

Analysis: $\quad$ Calc for $\mathrm{C}_{15} \mathrm{H}_{20} \mathrm{O}_{3} \mathrm{Si}(286.48)$

Calc. C $62.89 \%$; $10.55 \%$

Found: $\quad$ C $62.70 \%$; $\quad$ H $10.57 \%$

\section{(2S,5R)-1-tert-Butyldimethylsilyloxy-2,6-dimethyl-5-hydroxy-6-hepten-3-one (syn-28f)}

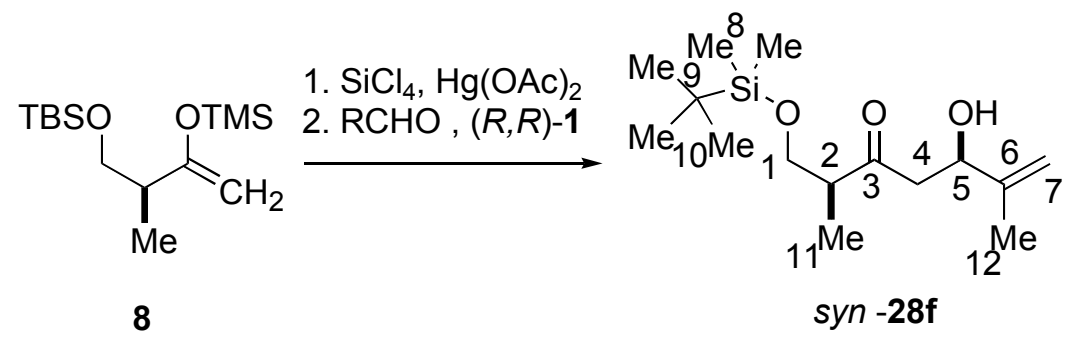

Following General Procedure III using 8 (288 mg, $1.00 \mathrm{mmol}), \mathrm{SiCl}_{4}(0.23 \mathrm{~mL}, 2.00$ $\mathrm{mmol}), \mathrm{Hg}(\mathrm{OAc})_{2}(3.2 \mathrm{mg}, 0.01 \mathrm{mmol})$, methacrolein $(70 \mathrm{mg}, 1.00 \mathrm{mmol}),(R, R)-\mathbf{1}(37 \mathrm{mg}, 0.10$ $\mathrm{mmol})$ in $2 \mathrm{~mL}$ of $\mathrm{CH}_{2} \mathrm{Cl}_{2}, 232 \mathrm{mg}(0.81 \mathrm{mmol}, 81 \%)$ of syn-28f was obtained as colorless viscous oil after column chromatography (silica gel, hexane/EtOAc, 5/1). 
Data for syn-28f:

${ }^{1} \mathrm{H}$ NMR: $\quad\left(500 \mathrm{MHz}, \mathrm{CDCl}_{3}\right)$;

$5.01\left(\mathrm{~s}, 1 \mathrm{H}, \mathrm{CH}_{2}\right) ; 4.86\left(\mathrm{~s}, 1 \mathrm{H}, \mathrm{CH}_{2}\right) ; 4.51(\mathrm{~d}, J=9.5,1 \mathrm{H}, \mathrm{CHOH}) ; 3.65-3.74$ $\left(\mathrm{ABX}, 2 \mathrm{H}, \mathrm{TBSOCH}_{2}\right) ; 3.17(\mathrm{~d}, J=3.2,1 \mathrm{H}, \mathrm{OH}) ; 2.80(\mathrm{~m}, 1 \mathrm{H}, \mathrm{COCH}) ; 2.67$ $2.82\left(\mathrm{ABX}, 2 \mathrm{H}, \mathrm{COCH}_{2}\right) ; 1.75\left(\mathrm{~s}, 3 \mathrm{H}, \mathrm{CH}_{3}\right) ; 1.03\left(\mathrm{~d}, \mathrm{~J}=6.8,3 \mathrm{H}, \mathrm{CHCH}_{3}\right) ; 0.87$ (s, 9H, $\left.\left(\mathrm{CH}_{3}\right)_{3} \mathrm{CSi}\right) ; 0.04\left(\mathrm{~d}, J=5.8,6 \mathrm{H}, \mathrm{SiCH}_{3}\right)$

${ }^{13} \mathrm{C} \mathrm{NMR}: \quad\left(101 \mathrm{MHz}, \mathrm{CDCl}_{3}\right)$

$214.9(\mathrm{C}(3)) ; 145.7(\mathrm{C}(6)) ; 111.0(\mathrm{C}(7)) ; 70.8(\mathrm{C}(5)) ; 65.6(\mathrm{C}(1)) ; 49.0(\mathrm{C}(2))$; $48.1(\mathrm{C}(4)) ; 25.8(\mathrm{C}(10)) ; 18.4(\mathrm{C}(12)) ; 18.3(\mathrm{C}(9)) 12.6(\mathrm{C}(11)) ;-5.6,-5.7(\mathrm{C}(8))$

IR: (neat)

3467 (m, br); 3078 (m); 2955 (s); 2932 (s); 2886 (m); 2859 (m); 1709 (s); 1653 (w); 1463 (m); $1388(\mathrm{~m}) ; 1362(\mathrm{w}) ; 1257$ (m); 1099 (s); 1007 (m)

$\underline{\mathrm{MS}}: \quad(\mathrm{FI})$

$287(1) ; 286\left(1, \mathrm{M}^{+}\right) ; 231$ (6); 229 (100); 211 (2); 159 (4); 111 (1); $111(2) ; 110$ (2); $110(2) ; 110(2) ; 110(1)$

Opt. rot. : $\quad+76.3\left(\mathrm{CHCl}_{3}, \mathrm{c}=0.35\right)$

TLC: $\quad R_{f}=0.17$ (hexane/EtOAc, $\left.5 / 1, \mathrm{SiO}_{2}\right)$ [p-anisaldehyde]

SFC: $\quad$ (5-benzoyloxy-28f, Chiralpak OD column, 150 bar, $3.0 \mathrm{~mL} / \mathrm{min}, 1.5 \% \mathrm{MeOH}$ ): $t_{R}(2 S, 5 S)$-anti-5-benzoyloxy-28f $5.26 \min (6 \%) ;(2 S, 5 R)$-syn-5-benzoyloxy-28f $4.44 \min (94 \%)$

Analysis: $\quad$ Calc for $\mathrm{C}_{15} \mathrm{H}_{20} \mathrm{O}_{3} \mathrm{Si}$ (286.48)

Calc. $\quad$ C $62.89 \%$; $\quad$ H $10.55 \%$

Found: $\quad$ C $62.81 \%$; H $10.68 \%$ 


\section{(2S,5S)-1-tert-Butyldimethylsilyloxy-2,6-dimethyl-5-hydroxy-6-hepten-3-one (anti-28f)}

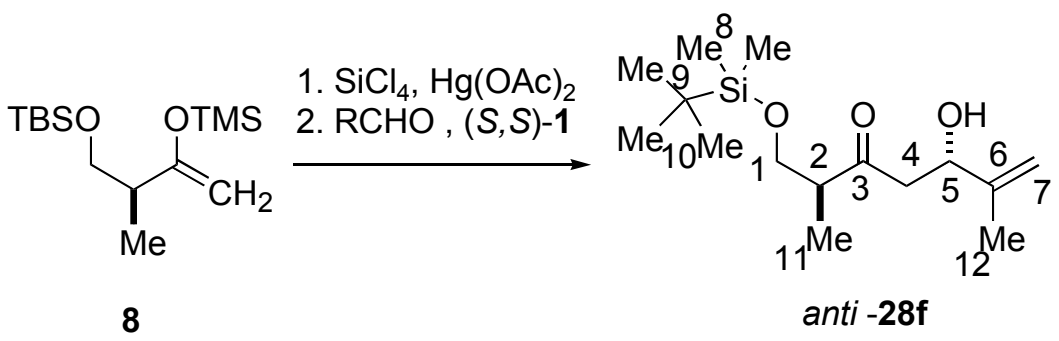

Following General Procedure III using 8 (288 mg, $1.00 \mathrm{mmol}), \mathrm{SiCl}_{4}(0.23 \mathrm{~mL}, 2.00$ mmol), $\mathrm{Hg}(\mathrm{OAc})_{2}(3.2 \mathrm{mg}, 0.01 \mathrm{mmol})$, methacrolein (70 mg, $\left.1.00 \mathrm{mmol}\right),(S, S)-1$ (37 mg, 0.10 $\mathrm{mmol})$ in $2 \mathrm{~mL}$ of $\mathrm{CH}_{2} \mathrm{Cl}_{2}, 240 \mathrm{mg}(0.84 \mathrm{mmol}, 84 \%)$ of anti-28f was obtained as colorless viscous oil after column chromatography (silica gel, pentane/ether, 3/1).

Data for anti-28f:

${ }^{1} \mathrm{H}$ NMR: $\quad\left(500 \mathrm{MHz}, \mathrm{CDCl}_{3}\right)$;

$5.01\left(\mathrm{~m}, 1 \mathrm{H}, \mathrm{CH}_{2}\right) ; 4.86\left(\mathrm{t}, J=1.2,1 \mathrm{H}, \mathrm{CH}_{2}\right) ; 4.49(\mathrm{~m}, 1 \mathrm{H}, \mathrm{CHOH}) ; 3.65-3.76$ $\left(\mathrm{ABX}, 2 \mathrm{H}, \mathrm{TBSOCH}_{2}\right) ; 3.21(\mathrm{~d}, J=3.2,1 \mathrm{H}, \mathrm{OH}) ; 2.80(\mathrm{~m}, 1 \mathrm{H}, \mathrm{COCH}) ; 2.67-$ $2.75\left(\mathrm{ABX}, 2 \mathrm{H}, \mathrm{COCH}_{2}\right) ; 1.75\left(\mathrm{~s}, 3 \mathrm{H}, \mathrm{CH}_{3}\right) ; 1.05\left(\mathrm{~d}, \mathrm{~J}=6.9,3 \mathrm{H}, \mathrm{CHCH}_{3}\right) ; 0.87$ (s, 9H, $\left.\left(\mathrm{CH}_{3}\right)_{3} \mathrm{CSi}\right) ; 0.04\left(\mathrm{~d}, J=3.6,6 \mathrm{H}, \mathrm{SiCH}_{3}\right)$

${ }^{13} \mathrm{C} \mathrm{NMR}: \quad\left(126 \mathrm{MHz}, \mathrm{CDCl}_{3}\right)$

214.9 (C(3)); 145.7 (C(6)); 110.1 (C(7)); 71.2 (C(5)); 65.5 (C(1)); 49.2 (C(2)); 47.6 (C(4)); 25.8 (C(10)); 18.3 (C(12)); 18.2 (C(9)) 12.7 (C(11)); -5.6 (C(8))

IR: (neat)

3468 (m, br); 3078 (w); 2955 (s); 2932 (s); 2886 (m); 2859 (m); 1708 (s); 1653 (w); $1463(\mathrm{~m}) ; 1389(\mathrm{~m}) ; 1362(\mathrm{w}) ; 1257$ (m); $1100(\mathrm{~s}) ; 1007$ (m)

MS: $\quad(\mathrm{FI})$

287 (1); 286 (1, M+ $) ; 231$ (5); 230 (17); 229 (100); 229 (1); 211 (1); 159 (2); 111 (1); $110(1) ; 110(2) ; 110(2)$

Opt. rot.: $\quad-10.6\left(\mathrm{CHCl}_{3}, \mathrm{c}=0.35\right)$

TLC: $\quad R_{f}=0.24$ (pentane/ether, $3 / 1, \mathrm{SiO}_{2}$ ) [ $p$-anisaldehyde]

SFC: $\quad$ (5-benzoyloxy-28f, Chiralpak OD column, $150 \mathrm{bar}, 3.0 \mathrm{~mL} / \mathrm{min}, 1.5 \% \mathrm{MeOH}$ ): $t_{R}(2 S, 5 S)$-anti-5-benzoyloxy-28f $5.26 \min (89 \%) ;(2 S, 5 R)$-syn-5-benzoyloxy-28f 


\section{$4.44 \min (11 \%)$}

Analysis: $\quad$ Calc for $\mathrm{C}_{15} \mathrm{H}_{20} \mathrm{O}_{3} \mathrm{Si}(286.48)$

Calc. $\quad$ C $62.89 \%$; $10.55 \%$

Found: $\quad$ C $62.50 \%$; H $10.51 \%$

\section{(2S,5R,6E)-1-tert-Butyldimethylsilyloxy-2,6-dimethyl-5-hydroxy-6-octen-3-one (syn-28g)}

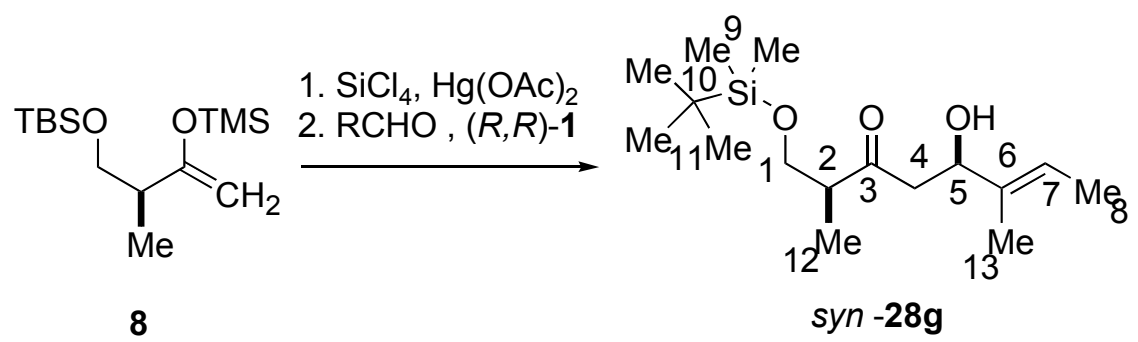

Following General Procedure III using 8 (288 mg, $1.00 \mathrm{mmol}), \mathrm{SiCl}_{4}(0.23 \mathrm{~mL}, 2.00$ $\mathrm{mmol}), \mathrm{Hg}(\mathrm{OAc})_{2}(3.2 \mathrm{mg}, 0.01 \mathrm{mmol})$, tiglic aldehyde $(84 \mathrm{mg}, 1.00 \mathrm{mmol}),(R, R)-1(37 \mathrm{mg}$, $0.10 \mathrm{mmol})$ in $2 \mathrm{~mL}$ of $\mathrm{CH}_{2} \mathrm{Cl}_{2}, 235 \mathrm{mg}(0.78 \mathrm{mmol}, 78 \%)$ of syn-28g was obtained as colorless viscous oil after column chromatography (silica gel, hexane/EtOAc, 5/1).

Data for syn-28g:

${ }^{1} \mathrm{H}$ NMR: $\quad\left(500 \mathrm{MHz}, \mathrm{CDCl}_{3}\right)$;

$5.53(\mathrm{qt}, J=6.5,1.2,1 \mathrm{H}, \mathrm{CH}) ; 4.46(\mathrm{td}, J=6.4,1.5,1 \mathrm{H}, \mathrm{CHOH}) ; 3.64-3.74$ $\left(\mathrm{ABX}, 2 \mathrm{H}, \mathrm{TBSOCH}_{2}\right) ; 3.06(\mathrm{~d}, J=2.7,1 \mathrm{H}, \mathrm{OH}) ; 2.79(\mathrm{~m}, 1 \mathrm{H}, \mathrm{COCH}) ; 2.64-$ $2.76\left(\mathrm{ABX}, 2 \mathrm{H}, \mathrm{COCH}_{2}\right) ; 1.63\left(\mathrm{~d}, J=1.0,3 \mathrm{H}, \mathrm{CH}_{3}\right) ; 1.61\left(\mathrm{~d}, J=6.6,3 \mathrm{H}, \mathrm{CH}_{3}\right)$; $1.03\left(\mathrm{~d}, J=7.1,3 \mathrm{H}, \mathrm{CHCH}_{3}\right) ; 0.87\left(\mathrm{~s}, 9 \mathrm{H},\left(\mathrm{CH}_{3}\right)_{3} \mathrm{CSi}\right) ; 0.03(\mathrm{~d}, J=6.9,6 \mathrm{H}$, $\left.\mathrm{SiCH}_{3}\right)$

${ }^{13} \mathrm{C} \mathrm{NMR}: \quad\left(126 \mathrm{MHz}, \mathrm{CDCl}_{3}\right)$

$215.1(\mathrm{C}(3)) ; 136.1(\mathrm{C}(6)) ; 120.6(\mathrm{C}(7)) ; 72.6(\mathrm{C}(5)) ; 65.6(\mathrm{C}(1)) ; 49.0(\mathrm{C}(2))$; $48.1(\mathrm{C}(4))$; 25.8 (C(11)); 18.2 (C(10)); $13.0(\mathrm{C}(8)) 12.6$ (C(13)); 11.7 (C(12)); $5.6,-5.6(\mathrm{C}(9))$

IR: (neat)

3446 (m, br); 2956 (s); 2931 (s); 2886 (m); 2860 (m); 1709 (s); 1471 (m); 1387 (m); $1362(\mathrm{w}) ; 1257(\mathrm{~m}) ; 1099(\mathrm{~s}) ; 1008$ (m) 
MS: $\quad(\mathrm{FI})$

302 (5); 301 (17); 300 (75, M+); 245 (5); 244 (19); 243 (100); 225 (3); 159 (9);

$104(2) ; 84(3)$

Opt. rot. : $\quad+48.6\left(\mathrm{CHCl}_{3}, \mathrm{c}=1.0\right)$

TLC: $\quad R_{f}=0.17$ (hexane/EtOAc, $\left.5 / 1, \mathrm{SiO}_{2}\right)$ [ $p$-anisaldehyde]

SFC: $\quad$ (5-benzoyloxy-28g, Chiralpak OD column, $150 \mathrm{bar}, 3.0 \mathrm{~mL} / \mathrm{min}, 2.5 \% \mathrm{MeOH}$ ):

$t_{R}(2 S, 5 R)$-syn-5-benzoyloxy-28g $2.94 \min (96 \%)$; (2S, 5S)-anti-5-benzoyloxy-

28g $3.45 \min (4 \%)$

Analysis: $\quad$ Calc for $\mathrm{C}_{15} \mathrm{H}_{20} \mathrm{O}_{3} \mathrm{Si}(300.51)$

Calc. $\quad$ C $63.95 \%$; $10.73 \%$

Found: $\quad$ C $64.04 \%$; H $10.86 \%$

\section{(2S,5S,6E)-1-tert-Butyldimethylsilyloxy-2,6-dimethyl-5-hydroxy-6-octen-3-one (anti-28g)}

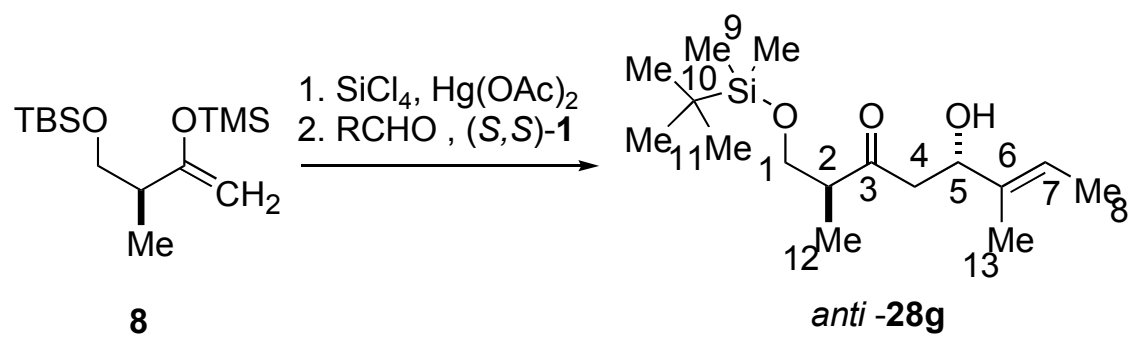

Following General Procedure III using 8 (288 mg, $1.00 \mathrm{mmol}), \mathrm{SiCl}_{4}(0.23 \mathrm{~mL}, 2.00$ $\mathrm{mmol}), \mathrm{Hg}(\mathrm{OAc})_{2}(3.2 \mathrm{mg}, 0.01 \mathrm{mmol})$, tiglic aldehyde $(84 \mathrm{mg}, 1.00 \mathrm{mmol}),(S, S)-1$ (37 mg, $0.10 \mathrm{mmol})$ in $2 \mathrm{~mL}$ of $\mathrm{CH}_{2} \mathrm{Cl}_{2}, 226 \mathrm{mg}(0.75 \mathrm{mmol}, 75 \%)$ of anti-28g was obtained as colorless viscous oil after column chromatography (silica gel, hexane/EtOAc, 5/1).

Data for anti-28g:

${ }^{1} \mathrm{H}$ NMR: $\quad\left(500 \mathrm{MHz}, \mathrm{CDCl}_{3}\right)$;

$5.54(\mathrm{qd}, J=6.7,1.0,1 \mathrm{H}, \mathrm{CH}) ; 4.44(\mathrm{~d}, J=9.8,1 \mathrm{H}, \mathrm{CHOH}) ; 3.64-3.76(\mathrm{ABX}$, $\left.2 \mathrm{H}, \mathrm{TBSOCH}_{2}\right) ; 3.11(\mathrm{~d}, J=2.7,1 \mathrm{H}, \mathrm{OH}) ; 2.79(\mathrm{~m}, 1 \mathrm{H}, \mathrm{COCH}) ; 2.64-2.80$ $\left(\mathrm{ABX}, 2 \mathrm{H}, \mathrm{COCH}_{2}\right) ; 1.63\left(\mathrm{~d}, J=1.0,3 \mathrm{H}, \mathrm{CH}_{3}\right) ; 1.61\left(\mathrm{~d}, J=6.8,3 \mathrm{H}, \mathrm{CH}_{3}\right) ; 1.05$ $\left(\mathrm{d}, J=7.0,3 \mathrm{H}, \mathrm{CHCH}_{3}\right) ; 0.88\left(\mathrm{~s}, 9 \mathrm{H},\left(\mathrm{CH}_{3}\right)_{3} \mathrm{CSi}\right) ; 0.04\left(\mathrm{~d}, J=3.7,6 \mathrm{H}, \mathrm{SiCH}_{3}\right)$ 
${ }^{13} \mathrm{C} \mathrm{NMR}: \quad\left(126 \mathrm{MHz}, \mathrm{CDCl}_{3}\right)$

$215.2(\mathrm{C}(3)) ; 136.2(\mathrm{C}(6)) ; 120.7(\mathrm{C}(7)) ; 73.0(\mathrm{C}(5)) ; 65.5(\mathrm{C}(1)) ; 49.2(\mathrm{C}(2))$;

$47.7(\mathrm{C}(4))$; 25.8 (C(11)); 18.2 (C(10)); 13.0 (C(8)) 12.7 (C(13)); 11.7 (C(12)); $5.6(\mathrm{C}(9))$

IR: (neat)

3467 (m, br); 2955 (s); 2931 (s); 2886 (m); 2860 (m); 1708 (s); 1463 (m); 1387 (m); $1362(\mathrm{w}) ; 1256(\mathrm{~m}) ; 1100(\mathrm{~s}) ; 1008(\mathrm{~m})$

MS: $\quad(\mathrm{FI})$

314 (4); 302 (5); 301 (18); $300\left(74, \mathrm{M}^{+}\right) ; 245$ (5); 244 (20); 243 (100); 225 (4); $160(2) ; 159$ (17); $104(2) ; 84$ (5)

Opt. rot. : $\quad+4.96\left(\mathrm{CHCl}_{3}, \mathrm{c}=0.75\right)$

TLC: $\quad R_{f}=0.20$ (hexane/EtOAc, $5 / 1, \mathrm{SiO}_{2}$ ) [ $p$-anisaldehyde]

SFC: $\quad$ (5-benzoyloxy-28g, Chiralpak OD column, $150 \mathrm{bar}, 3.0 \mathrm{~mL} / \mathrm{min}, 2.5 \% \mathrm{MeOH}$ ):

$t_{R}(2 S, 5 R)$-syn-5-benzoyloxy-28g $3.03 \min (18 \%)$; (2S, 5S)-anti-5-benzoyloxy28g $3.48 \min (82 \%)$

Analysis: $\quad$ Calc for $\mathrm{C}_{15} \mathrm{H}_{20} \mathrm{O}_{3} \mathrm{Si}(300.51)$

Calc. $\quad$ C $63.95 \%$; $10.73 \%$

Found: $\quad$ C $63.74 \%$; H $10.87 \%$

(2S,5R)-1-tert-Butyldimethylsilyloxy-2-methyl-5-hydroxy-7-phenyl-6-heptyn-3-one (syn28h)

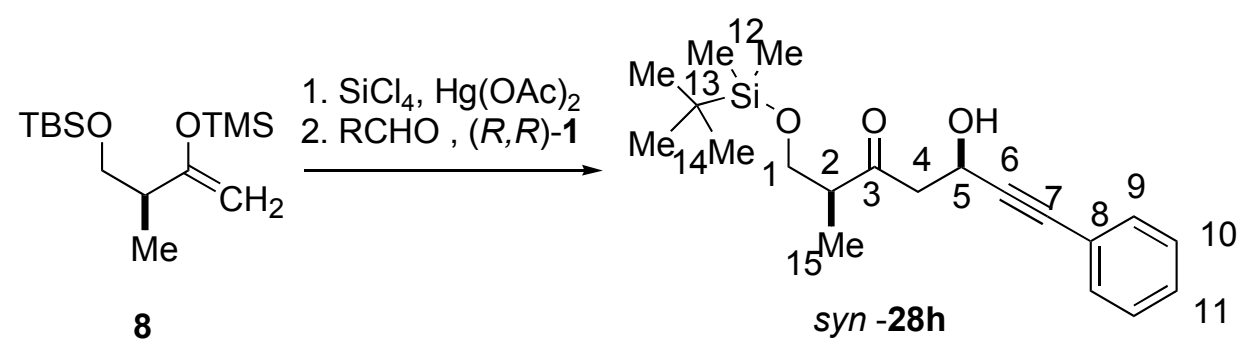

Following the general procedure III using $8(288 \mathrm{mg}, 1.00 \mathrm{mmol}), \mathrm{SiCl}_{4}(0.23 \mathrm{~mL}, 2.00$ $\mathrm{mmol}), \mathrm{Hg}(\mathrm{OAc})_{2}(3.2 \mathrm{mg}, 0.01 \mathrm{mmol})$, phenylpropargyl aldehyde (130 mg, $\left.1.00 \mathrm{mmol}\right),(R, R)-\mathbf{1}$ (37 mg, $0.10 \mathrm{mmol})$ in $2 \mathrm{~mL}$ of $\mathrm{CH}_{2} \mathrm{Cl}_{2}, 288 \mathrm{mg}(0.83 \mathrm{mmol}, 83 \%)$ of syn-28h was obtained as 
colorless viscous oil after column chromatography (silica gel, hexane/EtOAc, 5/1).

Data for syn-28h:

매 NMR: $\quad\left(500 \mathrm{MHz}, \mathrm{CDCl}_{3}\right)$

7.28 - 7.43 (m, 5H, aromatic); 5.01 - 5.06 (m, 1H, CHOH); 3.67 - 3.77 (ABX, 2H,TBSOCH$\left.{ }_{2}\right) ; 3.33(\mathrm{~d}, J=5.1,1 \mathrm{H}, \mathrm{OH}) ; 2.96-3.13\left(\mathrm{ABX}, 2 \mathrm{H}, \mathrm{COCH}_{2}\right) ; 2.82$ $(\mathrm{m}, 1 \mathrm{H}, \mathrm{COCH}) ; 1.08\left(\mathrm{~d}, J=7.1,3 \mathrm{H}, \mathrm{CHCH}_{3}\right) ; 0.88\left(\mathrm{~s}, 9 \mathrm{H},\left(\mathrm{CH}_{3}\right)_{3} \mathrm{CSi}\right) ; 0.06(\mathrm{~d}, J$ $\left.=5.1,6 \mathrm{H}, \mathrm{SiCH}_{3}\right)$

${ }^{13} \mathrm{C} \mathrm{NMR}: \quad\left(126 \mathrm{MHz}, \mathrm{CDCl}_{3}\right)$

213.2 (C(3)); 131.7 (C(9)); 128.4 (C(11)); 128.2 (C(10)); 122.4 (C(8)); 88.5 $(\mathrm{C}(6)) ; 84.6(\mathrm{C}(7)) ; 65.3(\mathrm{C}(5)) ; 58.8(\mathrm{C}(1)) ; 49.4(\mathrm{C}(2)) ; 49.0(\mathrm{C}(4)) ; 25.7$ $(\mathrm{C}(14)) ; 18.1$ (C(13)); $12.4(\mathrm{C}(15)) ;-5.7,-5.7$ (C(12))

IR: (neat) 3419 (m, br); 3075(w); 2955 (s); 2931 (s); 2885 (m); 2859 (m); 2250 (w); 1711 (s); 1491 (m); 1471 (m); 1389 (m); 1362 (m); 1256 (m); 1102 (s); 1077 (s); 1029 (m)

$\underline{\mathrm{MS}}: \quad(\mathrm{FI})$

347 (6); $346\left(11, \mathrm{M}^{+}\right) ; 328$ (3); 291 (6); 290 (23); 289 (100); 271 (9); 159 (6); 145 (10); $130(5)$

Opt. rot. : $\quad+37.6\left(\mathrm{CHCl}_{3}, \mathrm{c}=2.1\right)$

TLC: $\quad R_{f}=0.16\left(\right.$ hexane/EtOAc, $\left.5 / 1, \mathrm{SiO}_{2}\right)$ [ $p$-anisaldehyde]

SFC: $\quad$ (Chiralpak OD column, $150 \mathrm{bar}, 3.0 \mathrm{~mL} / \mathrm{min}, 7.5 \% \mathrm{MeOH}$ ):

$t_{R}(2 S, 5 R)$-syn-28h $3.35 \min (76 \%)$; (2S, 5S)-anti-28h 3.82 min (24\%)

Analysis: $\quad$ Calc for $\mathrm{C}_{20} \mathrm{H}_{30} \mathrm{O}_{3} \mathrm{Si}$ (346.54)

Calc. $\quad$ C $69.32 \%$; $\quad$ H $8.73 \%$

Found: $\quad$ C $69.20 \%$; $\quad$ H $8.77 \%$ 


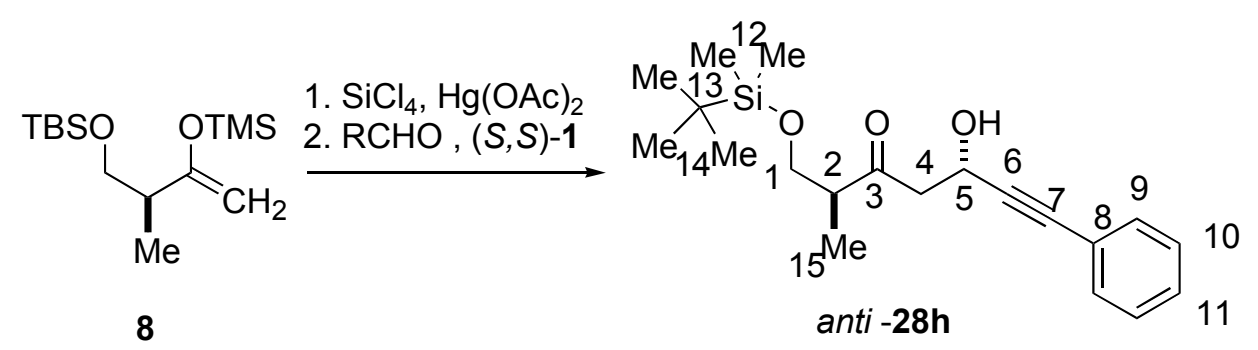

Following General Procedure III using 8 (288 mg, $1.00 \mathrm{mmol}), \mathrm{SiCl}_{4}(0.23 \mathrm{~mL}, 2.00$ $\mathrm{mmol}), \mathrm{Hg}(\mathrm{OAc})_{2}(3.2 \mathrm{mg}, 0.01 \mathrm{mmol})$, phenylpropargyl aldehyde (130 mg, $\left.1.00 \mathrm{mmol}\right),(S, S)-1$ (37 mg, $0.10 \mathrm{mmol})$ in $2 \mathrm{~mL}$ of $\mathrm{CH}_{2} \mathrm{Cl}_{2}, 282 \mathrm{mg}(0.82 \mathrm{mmol}, 82 \%)$ of anti-28h was obtained as colorless viscous oil after column chromatography (silica gel, hexane/EtOAc, 5/1).

Data for anti-28h:

${ }^{1} \mathrm{H}$ NMR: $\quad\left(500 \mathrm{MHz}, \mathrm{CDCl}_{3}\right)$ $7.28-7.43$ (m, 5H, aromatic); $5.01-5.06(\mathrm{~m}, 1 \mathrm{H}, \mathrm{CHOH}) ; 3.67$ - 3.79 (ABX, 2H,TBSOCH$\left.{ }_{2}\right) ; 3.29(\mathrm{~d}, J=5.1,1 \mathrm{H}, \mathrm{OH}) ; 2.97-3.13\left(\mathrm{ABX}, 2 \mathrm{H}, \mathrm{COCH}_{2}\right) ; 2.82$ $(\mathrm{m}, 1 \mathrm{H}, \mathrm{COCH}) ; 1.09\left(\mathrm{~d}, J=7.1,3 \mathrm{H}, \mathrm{CHCH}_{3}\right) ; 0.88\left(\mathrm{~s}, 9 \mathrm{H},\left(\mathrm{CH}_{3}\right)_{3} \mathrm{CSi}\right) ; 0.05(\mathrm{~d}, J$ $\left.=3.9,6 \mathrm{H}, \mathrm{SiCH}_{3}\right)$

${ }^{13} \mathrm{C} \mathrm{NMR}: \quad\left(126 \mathrm{MHz}, \mathrm{CDCl}_{3}\right)$

213.1 (C(3)); 131.7 (C(9)); 128.4 (C(11)); 128.2 (C(10)); 122.4 (C(8)); 88.5 $(\mathrm{C}(6)) ; 84.6$ (C(7)); $65.2(\mathrm{C}(5)) ; 59.0(\mathrm{C}(1)) ; 49.2(\mathrm{C}(2)) ; 49.0$ (C(4)); 25.8 $(\mathrm{C}(14)) ; 18.1(\mathrm{C}(13)) ; 12.6(\mathrm{C}(15)) ;-5.7$ (C(12))

IR: $\quad$ (net)

3427 (m, br); 3059(w); 2955 (s); 2930 (s); 2885 (m); 2858 (m); 2249 (w); 1710 (s); 1599 (w); 1491 (m); 1471 (m); 1389 (m); 1362 (m); 1256 (m); 1102 (s); 1078 (s); 1029 (m)

MS: $\quad(\mathrm{FI})$

348 (2); 347 (7); 346 (9, M+); 328 (2); 291 (6); 290 (24); 289 (100); 271 (6); 159 (3); $145(2) ; 145(7) ; 130(3)$

Opt. rot. : $\quad+14.1\left(\mathrm{CHCl}_{3}, \mathrm{c}=2.1\right)$ 
TLC: $\quad R_{f}=0.14$ (hexane/EtOAc, $5 / 1, \mathrm{SiO}_{2}$ ) [p-anisaldehyde]

SFC: (Chiralpak OD column, $150 \mathrm{bar}, 3.0 \mathrm{~mL} / \mathrm{min}, 7.5 \% \mathrm{MeOH}$ ):

$t_{R}(2 S, 5 R)$-syn-28h $3.47 \min (43 \%)$; (2S, 5S)-anti-28h $3.94 \min (57 \%)$

Analysis: $\quad$ Calc for $\mathrm{C}_{20} \mathrm{H}_{30} \mathrm{O}_{3} \mathrm{Si}(346.54)$

Calc. $\quad$ C $69.32 \% ; \quad \mathrm{H} 8.73 \%$

Found: $\quad$ C $69.13 \%$; H $8.75 \%$

(2S,5R)-1-tert-Butyldimethylsilyloxy-2-methyl-5-hydroxy-7-phenyl-3-heptanone (syn-28i)

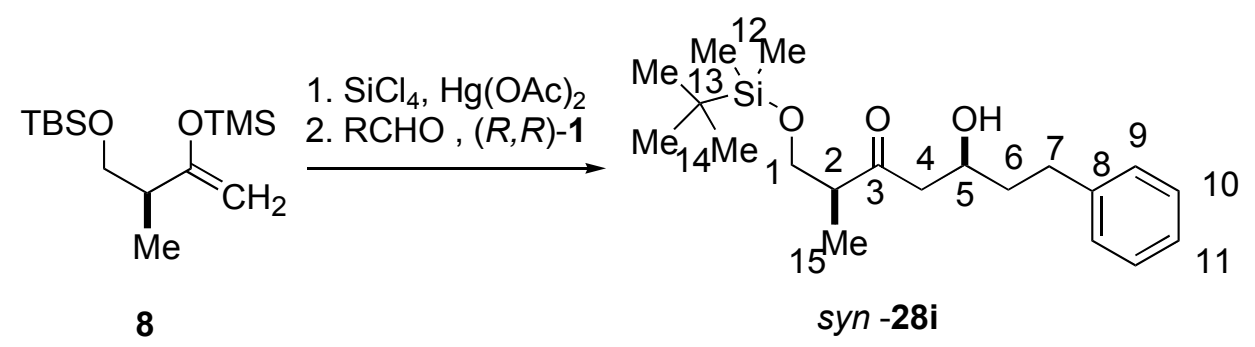

Following the general procedure III using $8(288 \mathrm{mg}, 1.00 \mathrm{mmol}), \mathrm{SiCl}_{4}(0.23 \mathrm{~mL}, 2.00$ $\mathrm{mmol}), \mathrm{Hg}(\mathrm{OAc})_{2}(3.2 \mathrm{mg}, 0.01 \mathrm{mmol})$, hydrocinnamaldehyde (134 mg, $\left.1.00 \mathrm{mmol}\right),(R, R)-\mathbf{1}(37$ $\mathrm{mg}, 0.10 \mathrm{mmol})$ in $2 \mathrm{~mL}$ of $\mathrm{CH}_{2} \mathrm{Cl}_{2}, 120 \mathrm{mg}(0.34 \mathrm{mmol}, 34 \%)$ of syn-28i was obtained as colorless viscous oil after column chromatography (silica gel, hexane/EtOAc, 5/1). Data for syn-28i:

1 $\mathrm{H}$ NMR: $\quad\left(500 \mathrm{MHz}, \mathrm{CDCl}_{3}\right)$

$7.17-7.30(\mathrm{~m}, 5 \mathrm{H}$, aromatic); $4.07(\mathrm{~m}, 1 \mathrm{H}, \mathrm{CHOH}) ; 3.62-3.73(\mathrm{ABX}$, $\left.2 \mathrm{H}, \mathrm{TBSOCH}_{2}\right) ; 3.24(\mathrm{dd}, J=3.5,0.8,1 \mathrm{H}, \mathrm{OH}) ; 2.56-2.85\left(\mathrm{~m}, 5 \mathrm{H}, \mathrm{COCH}_{2}\right.$, $\left.\mathrm{COCH}, \mathrm{PhCH}_{2}\right) ; 1.65-1.86\left(2 \mathrm{~m}, 2 \mathrm{H}, \mathrm{CH}_{2}\right) ; 1.02\left(\mathrm{~d}, J=7.0,3 \mathrm{H}, \mathrm{CHCH}_{3}\right) ; 0.86$ (s, 9H, $\left.\left(\mathrm{CH}_{3}\right)_{3} \mathrm{CSi}\right) ; 0.03$ (d, $J=9.0,6 \mathrm{H}, \mathrm{SiCH}_{3}$ )

${ }^{13} \mathrm{C}$ NMR: $\quad\left(126 \mathrm{MHz}, \mathrm{CDCl}_{3}\right)$

$215.4(\mathrm{C}(3)) ; 141.9(\mathrm{C}(8)) ; 128.4(\mathrm{C}(10)) ; 128.3(\mathrm{C}(11)) ; 125.8(\mathrm{C}(9)) ; 66.7$ $(\mathrm{C}(5)) ; 65.6(\mathrm{C}(1)) ; 49.3(\mathrm{C}(2)) ; 48.8(\mathrm{C}(4)) ; 38.0(\mathrm{C}(6)) ; 31.7(\mathrm{C}(7)) ; 25.7$ $(\mathrm{C}(14)) ; 18.1(\mathrm{C}(13)) ; 12.6(\mathrm{C}(15)) ;-5.7,5.7(\mathrm{C}(12))$

IR: (neat)

3467 (m, br); 3063(w); 3028 (w); 2953 (s); 2930 (s); 2858 (m); 1707 (s); 1604 
(w); 1496 (m); 1462 (m); 1388 (m); 1256 (m); 1094 (s)

MS: $\quad(\mathrm{FI})$

351 (3); 350 (5, M+ ); 295 (6); 294 (22); 293 (100); 275 (6); 159 (4); 147 (7); 134

Opt. rot. : $\quad+46.3\left(\mathrm{CHCl}_{3}, \mathrm{c}=1.5\right)$

TLC: $\quad R_{f}=0.14\left(\right.$ hexane/EtOAc, $\left.5 / 1, \mathrm{SiO}_{2}\right)[p$-anisaldehyde]

SFC: $\quad$ (Chiralpak OD column, $150 \mathrm{bar}, 3.0 \mathrm{~mL} / \mathrm{min}, 5 \% \mathrm{MeOH}$ ):

$t_{R}(2 S, 5 R)-s y n-28 \mathbf{i} 3.45 \min (91 \%) ;(2 S, 5 S)$-anti-28i $3.87 \min (9 \%)$

Analysis: $\quad$ Calc for $\mathrm{C}_{20} \mathrm{H}_{34} \mathrm{O}_{3} \mathrm{Si}(350.57)$

Calc. $\quad \mathrm{C} 68.52 \%$; $9.78 \%$

Found: $\quad$ C $68.59 \%$; $\quad$ H $9.91 \%$

\section{(2S,5S)-1-tert-Butyldimethylsilyloxy-2-methyl-5-hydroxy-7-phenyl-3-heptanone (anti-28i)}

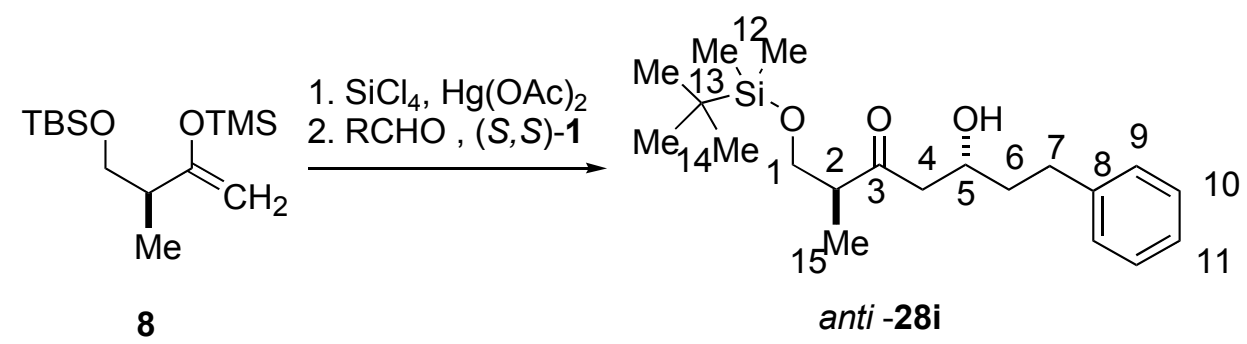

Following General Procedure III using 8 (288 mg, $1.00 \mathrm{mmol}), \mathrm{SiCl}_{4}(0.23 \mathrm{~mL}, 2.00$ $\mathrm{mmol}), \mathrm{Hg}(\mathrm{OAc})_{2}(3.2 \mathrm{mg}, 0.01 \mathrm{mmol})$, hydrocinnamaldehyde (134 mg, $\left.1.00 \mathrm{mmol}\right),(S, S)-1$ (37 $\mathrm{mg}, 0.10 \mathrm{mmol})$ in $2 \mathrm{~mL}$ of $\mathrm{CH}_{2} \mathrm{Cl}_{2}, 76 \mathrm{mg}(0.22 \mathrm{mmol}, 22 \%)$ of anti-28i was obtained as colorless viscous oil after column chromatography (silica gel, hexane/EtOAc, 5/1).

Data for anti-28i:

${ }^{1} \mathrm{H}$ NMR: $\quad\left(500 \mathrm{MHz}, \mathrm{CDCl}_{3}\right)$

7.17 - $7.30(\mathrm{~m}, 5 \mathrm{H}$, aromatic); $4.03(\mathrm{~m}, 1 \mathrm{H}, \mathrm{CHOH}) ; 3.62$ - 3.75 (ABX, $\left.2 \mathrm{H}, \mathrm{TBSOCH}_{2}\right) ; 3.28(\mathrm{dd}, J=3.2,0.7,1 \mathrm{H}, \mathrm{OH}) ; 2.56-2.85\left(\mathrm{~m}, 5 \mathrm{H}, \mathrm{COCH}_{2}\right.$, $\left.\mathrm{COCH}, \mathrm{PhCH}_{2}\right) ; 1.66-1.87\left(2 \mathrm{~m}, 2 \mathrm{H}, \mathrm{CH}_{2}\right) ; 1.03\left(\mathrm{~d}, J=7.0,3 \mathrm{H}, \mathrm{CHCH}_{3}\right) ; 0.87$ (s, 9H, $\left.\left(\mathrm{CH}_{3}\right)_{3} \mathrm{CSi}\right) ; 0.04\left(\mathrm{~d}, J=3.2,6 \mathrm{H}, \mathrm{SiCH}_{3}\right)$ 
${ }^{13} \mathrm{C} \mathrm{NMR}: \quad\left(126 \mathrm{MHz}, \mathrm{CDCl}_{3}\right)$

215.7 (C(3)); $142.2(\mathrm{C}(8)) ; 128.7$ (C(10)); 128.6 (C(11)); 126.1 (C(9)); 67.3

$(\mathrm{C}(5)) ; 65.7$ (C(1)); $49.4(\mathrm{C}(2)) ; 49.3(\mathrm{C}(4)) ; 38.4(\mathrm{C}(6)) ; 32.0(\mathrm{C}(7)) ; 26.0$ (C(14)); 18.4 (C(13)); 13.0 (C(15)); -5.4 (C(12))

IR: (neat)

3467 (m, br); 3063(w); 3027 (w); 2953 (s); 2931 (s); 2859 (m); 1706 (s); 1471

(m); $1388(\mathrm{~m}) ; 1256(\mathrm{~m}) ; 1096(\mathrm{~s})$

MS: $\quad(\mathrm{FI})$

351 (3); $350\left(5, \mathrm{M}^{+}\right) ; 295$ (6); 294 (22); 293 (100); 275 (3); 159 (V); 147 (5); 134 (3)

Opt. rot. : $\quad+16.5\left(\mathrm{CHCl}_{3}, \mathrm{c}=0.55\right)$

TLC: $\quad R_{f}=0.16$ (hexane/EtOAc, $5 / 1, \mathrm{SiO}_{2}$ ) [ $p$-anisaldehyde]

SFC: $\quad$ (Chiralpak OD column, $150 \mathrm{bar}, 3.0 \mathrm{~mL} / \mathrm{min}, 5 \% \mathrm{MeOH}$ ):

$t_{R}(2 S, 5 R)-s y n-28 \mathbf{i} 3.51 \min (29 \%) ;(2 S, 5 S)$-anti-28i $3.88 \min (71 \%)$

Analysis: $\quad$ Calc for $\mathrm{C}_{20} \mathrm{H}_{34} \mathrm{O}_{3} \mathrm{Si}(350.57)$

Calc. $\quad \mathrm{C} 68.52 \%$; $9.78 \%$

Found: $\quad$ C $68.64 \%$; H $9.78 \%$

(2S,5R)-1-tert-Butyldimethylsilyloxy-2-methyl-5-hydroxy-5-cyclohexyl-3-pentanone (syn28j)

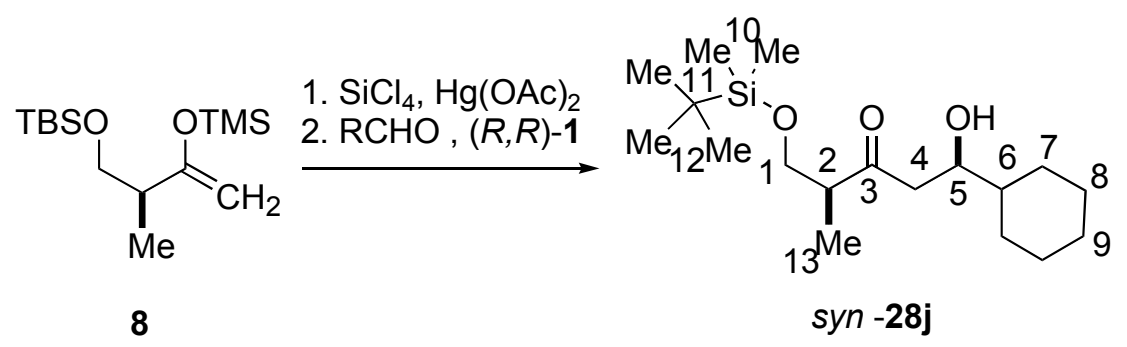

Following General Procedure III using 8 (288 mg, $1.00 \mathrm{mmol}), \mathrm{SiCl}_{4}(0.23 \mathrm{~mL}, 2.00$ $\mathrm{mmol}), \mathrm{Hg}(\mathrm{OAc})_{2}(3.2 \mathrm{mg}, 0.01 \mathrm{mmol})$, cyclohexanecarboxaldehyde (112 mg, $\left.1.00 \mathrm{mmol}\right)$, $(R, R)-1$ (37 mg, $0.10 \mathrm{mmol})$ in $2 \mathrm{~mL}$ of $\mathrm{CH}_{2} \mathrm{Cl}_{2}, 155 \mathrm{mg}(0.47 \mathrm{mmol}, 47 \%)$ of syn-28j was obtained as colorless viscous oil after column chromatography (silica gel, hexane/EtOAc, 5/1). 
Data for syn-28j:

${ }^{1} \mathrm{H}$ NMR: $\quad\left(500 \mathrm{MHz}, \mathrm{CDCl}_{3}\right)$

$3.83(\mathrm{~m}, 1 \mathrm{H}, \mathrm{CHOH}) ; 3.64-3.76\left(\mathrm{ABX}, 2 \mathrm{H}, \mathrm{TBSOCH}_{2}\right) ; 3.12(\mathrm{~d}, J=3.4,1 \mathrm{H}$, $\mathrm{OH}) ; 2.82(\mathrm{~m}, 1 \mathrm{H}, \mathrm{COCH}) ; 2.54-2.77\left(\mathrm{ABX}, 2 \mathrm{H}, \mathrm{COCH}_{2}\right) ; 1.66-1.89(5 \mathrm{H}$, $\left.\mathrm{CH}_{\mathrm{eq}}\right) ; 1.00-1.38\left(6 \mathrm{H}, \mathrm{CH}_{\mathrm{ax}}\right) ; 1.03\left(\mathrm{~d}, J=7.1,3 \mathrm{H}, \mathrm{CHCH}_{3}\right) ; 0.88(\mathrm{~s}, 9 \mathrm{H}$, $\left.\left(\mathrm{CH}_{3}\right)_{3} \mathrm{CSi}\right) ; 0.05\left(\mathrm{~d}, \mathrm{~J}=5.6,6 \mathrm{H}, \mathrm{SiCH}_{3}\right)$

${ }^{13} \mathrm{C} \mathrm{NMR}: \quad\left(126 \mathrm{MHz}, \mathrm{CDCl}_{3}\right)$

215.7 (C(3)); 71.3 (C(5)); 65.6 (C(1)); 48.9 (C(2)); 46.6 (C(4)); 42.9 (C(6)); 28.7, $28.3(\mathrm{C}(7)) ; 26.4(\mathrm{C}(9)) ; 26.2,26.0$ (C(8)) 25.7 (C(12)); 18.1 (C(11)); 12.7 $(\mathrm{C}(13)) ;-5.7(\mathrm{C}(10))$

IR: (neat)

3481 (m, br); 2929 (s); 2857 (m); 1707 (m); 1463 (m); 1388 (m); 1256 (m); 1099 (s)

MS: $\quad(\mathrm{FI})$

$329\left(0, \mathrm{M}^{+}\right) ; 273(6) ; 272(21) ; 271(100) ; 253(2) ; 159$ (2)

Opt. rot. : $\quad+49.1\left(\mathrm{CHCl}_{3}, \mathrm{c}=0.4\right)$

TLC: $\quad R_{f}=0.27$ (hexane/EtOAc, $5 / 1, \mathrm{SiO}_{2}$ ) [ $p$-anisaldehyde]

SFC: $\quad$ (5-benzoyloxy-28j, Chiralpak OD column, $150 \mathrm{bar}, 3.0 \mathrm{~mL} / \mathrm{min}, 2 \% \mathrm{MeOH}$ ): $t_{R}(2 S, 5 R)$-syn-5-benzoyloxy-28j $4.59 \min (94 \%)$; (2S, 5S)-anti-5-benzoyloxy-28j $5.52 \min (6 \%)$

Analysis: $\quad$ Calc for $\mathrm{C}_{18} \mathrm{H}_{36} \mathrm{O}_{3} \mathrm{Si}$ (328.56)

Calc. $\quad$ C $65.80 \%$; $\quad$ H $11.04 \%$

Found: $\quad$ C $65.92 \%$; H $11.26 \%$ 

28j)

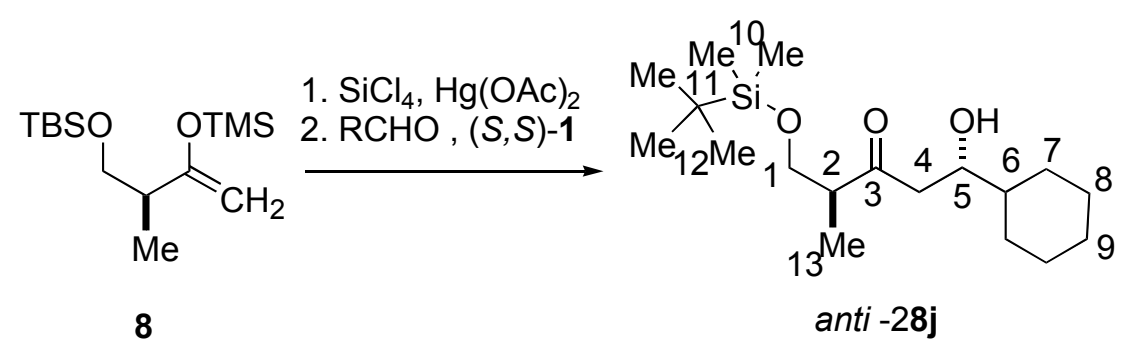

Following General Procedure III using $8(288 \mathrm{mg}, 1.00 \mathrm{mmol}), \mathrm{SiCl}_{4}(0.23 \mathrm{~mL}, 2.00$ $\mathrm{mmol}), \mathrm{Hg}(\mathrm{OAc})_{2}(3.2 \mathrm{mg}, 0.01 \mathrm{mmol})$, cyclohexanecarboxaldehyde $(112 \mathrm{mg}, 1.00 \mathrm{mmol})$, $(S, S)-\mathbf{1}(37 \mathrm{mg}, 0.10 \mathrm{mmol})$ in $2 \mathrm{~mL}$ of $\mathrm{CH}_{2} \mathrm{Cl}_{2}, 102 \mathrm{mg}(0.31 \mathrm{mmol}, 31 \%)$ of anti-28j was obtained as colorless viscous oil after column chromatography (silica gel, hexane/EtOAc, 5/1). Data for anti-28j:

${ }^{1} \mathrm{H}$ NMR: $\quad\left(500 \mathrm{MHz}, \mathrm{CDCl}_{3}\right)$

$3.81(\mathrm{~m}, 1 \mathrm{H}, \mathrm{CHOH}) ; 3.64-3.78\left(\mathrm{ABX}, 2 \mathrm{H}, \mathrm{TBSOCH}_{2}\right) ; 3.15(\mathrm{~d}, J=3.5,1 \mathrm{H}$, $\mathrm{OH}) ; 2.81(\mathrm{~m}, 1 \mathrm{H}, \mathrm{COCH}) ; 2.54-2.73\left(\mathrm{ABX}, 2 \mathrm{H}, \mathrm{COCH}_{2}\right) ; 1.66-1.89(5 \mathrm{H}$, $\left.\mathrm{CH}_{\text {eq }}\right) ; 0.97-1.42\left(6 \mathrm{H}, \mathrm{CH}_{\text {ax }}\right) ; 1.05\left(\mathrm{~d}, J=7.0,3 \mathrm{H}, \mathrm{CHCH}_{3}\right) ; 0.89(\mathrm{~s}, 9 \mathrm{H}$, $\left.\left(\mathrm{CH}_{3}\right)_{3} \mathrm{CSi}\right) ; 0.06\left(\mathrm{~d}, J=2.7,6 \mathrm{H}, \mathrm{SiCH}_{3}\right)$

${ }^{13} \mathrm{C} \mathrm{NMR}: \quad\left(126 \mathrm{MHz}, \mathrm{CDCl}_{3}\right)$ $215.9(\mathrm{C}(3)) ; 71.8(\mathrm{C}(5)) ; 65.5(\mathrm{C}(1)) ; 49.2(\mathrm{C}(2)) ; 46.1(\mathrm{C}(4)) ; 43.0(\mathrm{C}(6)) ; 28.8$, 28.2 (C(7)); $26.4(\mathrm{C}(9)) ; 26.2,26.1(\mathrm{C}(8)) 25.8$ (C(12)); 18.1 (C(11)); 12.8 $(\mathrm{C}(13)) ;-5.6,-5.7(\mathrm{C}(10))$

IR: (neat) 3502 (m, br); 2929 (s); 2857 (m); 1706 (m); 1463 (m); 1388 (m); 1256 (m); 1100 (s)

MS: $\quad(\mathrm{FI})$

$329\left(0, \mathrm{M}^{+}\right) ; 273(6) ; 272(21) ; 271(100) ; 253(2) ; 159$ (1)

Opt. rot. : $\quad-5.39^{\circ}\left(\mathrm{CHCl}_{3}, \mathrm{c}=0.3\right)$

TLC: $\quad R_{f}=0.32\left(\right.$ hexane/EtOAc, $\left.5 / 1, \mathrm{SiO}_{2}\right)[p$-anisaldehyde $]$

SFC: (5-benzoyloxy-28j, Chiralpak OD column, $150 \mathrm{bar}, 3.0 \mathrm{~mL} / \mathrm{min}, 2 \% \mathrm{MeOH}$ ): 
$t_{R}(2 S, 5 R)$-syn-5-benzoyloxy-28j $4.41 \min (20 \%) ;(2 S, 5 S)$-anti-5-benzoyloxy-28j

$5.17 \min (80 \%)$

Analysis: $\quad$ Calc for $\mathrm{C}_{18} \mathrm{H}_{36} \mathrm{O}_{3} \mathrm{Si}(328.56)$

Calc. $\quad$ C $65.80 \%$; $11.04 \%$

Found: $\quad$ C $65.80 \% ; \quad$ H $11.19 \%$

\section{(2S,5R)-1-tert-Butyldimethylsilyloxy-2,6,6-trimethyl-5-hydroxy-3-heptanone (syn-28k)}

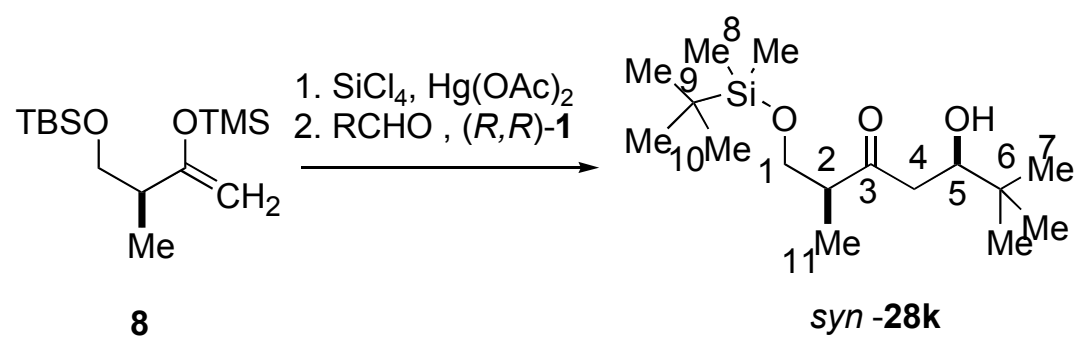

Following General Procedure III using 8 (288 mg, $1.00 \mathrm{mmol}), \mathrm{SiCl}_{4}(0.23 \mathrm{~mL}, 2.00$ mmol), $\mathrm{Hg}(\mathrm{OAc})_{2}(3.2 \mathrm{mg}, 0.01 \mathrm{mmol})$, pivaldehyde (86 mg, $\left.1.00 \mathrm{mmol}\right),(R, R)-1$ (37 mg, 0.10 $\mathrm{mmol})$ in $2 \mathrm{~mL}$ of $\mathrm{CH}_{2} \mathrm{Cl}_{2}, 220 \mathrm{mg}(0.73 \mathrm{mmol}, 73 \%)$ of syn-28k was obtained as colorless viscous oil after column chromatography (silica gel, pentane/ether, 3/1).

Data for syn-28k:

${ }^{1} \mathrm{H}$ NMR: $\quad\left(500 \mathrm{MHz}\right.$, benzene- $\left.d_{6}\right)$

$3.84(\mathrm{dt}, J=10.5,2.2,1 \mathrm{H}, \mathrm{CHOH}) ; 3.41-3.64\left(\mathrm{ABX}, 2 \mathrm{H}, \mathrm{TBSOCH}_{2}\right) ; 3.17(\mathrm{~d}, J$ $=2.9,1 \mathrm{H}, \mathrm{OH}) ; 2.37-2.59\left(\mathrm{ABX}, 2 \mathrm{H}, \mathrm{COCH}_{2}\right) ; 2.48(\mathrm{~m}, 1 \mathrm{H}, \mathrm{COCH}) ; 0.95(\mathrm{~s}$, 9H, $\left.\mathrm{C}\left(\mathrm{CH}_{2}\right)_{3}\right) ; 0.91\left(\mathrm{~s}, 9 \mathrm{H},\left(\mathrm{CH}_{3}\right)_{3} \mathrm{CSi}\right) ; 0.82\left(\mathrm{~d}, J=6.8,3 \mathrm{H}, \mathrm{CHCH}_{3}\right) ;-0.01(\mathrm{~d}, J$ $\left.=1.9,6 \mathrm{H}, \mathrm{SiCH}_{3}\right)$

${ }^{13} \mathrm{C} \mathrm{NMR}: \quad\left(126 \mathrm{MHz}, \mathrm{CDCl}_{3}\right)$

215.9 (C(3)); 74.4 (C(5)); 65.7 (C(1)); 48.9 (C(2)); 44.7 (C(4)); 34.0 (C(6)); 25.7 $(\mathrm{C}(7)) ; 25.6(\mathrm{C}(10)) ; 18.1(\mathrm{C}(9)) ; 12.7(\mathrm{C}(11)) ;-5.7(\mathrm{C}(8))$

IR: (neat)

3525 (m, br); 2957 (s); 2934 (s); 2861 (m); 1707 (m); 1471 (m); 1388 (m); 1364 (m); 1256 (m); 1087 (s); 1007 (m) 
MS: $\quad(\mathrm{FI})$

$303\left(1, \mathrm{M}^{+}\right) ; 247$ (6); $246(18) ; 245$ (100); 159 (1)

Opt. rot. : $\quad+45.0\left(\mathrm{CHCl}_{3}, \mathrm{c}=0.25\right)$

TLC: $\quad R_{f}=0.24$ (pentane/ether, $3 / 1, \mathrm{SiO}_{2}$ ) [p-anisaldehyde]

Analysis: $\quad$ Calc for $\mathrm{C}_{16} \mathrm{H}_{34} \mathrm{O}_{3} \mathrm{Si}$ (302.52)

Calc. $\quad$ C $63.52 \% ; \quad \mathrm{H} 11.33 \%$

Found: $\quad$ C $63.41 \%$; H $11.44 \%$

\section{(2S,5S)-1-tert-Butyldimethylsilyloxy-2,6,6-trimethyl-5-hydroxy-3-heptanone (anti-28k)}

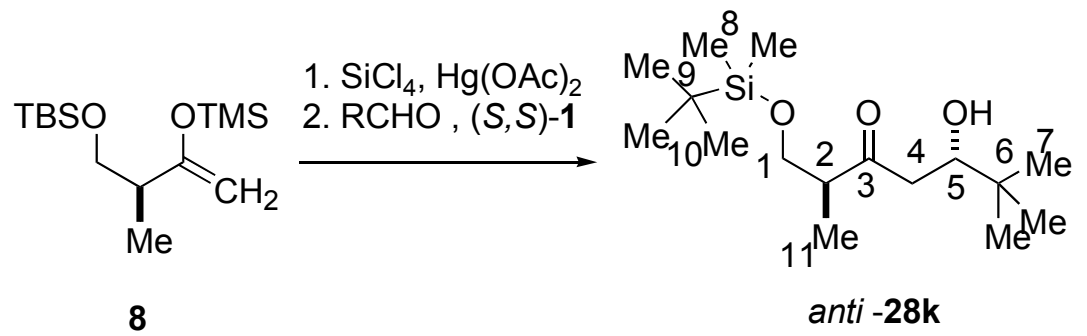

Following General Procedure III using 8 (288 mg, $1.00 \mathrm{mmol}), \mathrm{SiCl}_{4}(0.23 \mathrm{~mL}, 2.00$ mmol), $\mathrm{Hg}(\mathrm{OAc})_{2}(3.2 \mathrm{mg}, 0.01 \mathrm{mmol})$, pivaldehyde $(86 \mathrm{mg}, 1.00 \mathrm{mmol}),(S, S)-1$ (37 mg, 0.10 $\mathrm{mmol})$ in $2 \mathrm{~mL}$ of $\mathrm{CH}_{2} \mathrm{Cl}_{2}, 236 \mathrm{mg}(0.78 \mathrm{mmol}, 78 \%)$ of anti-28k was obtained as colorless viscous oil after column chromatography (silica gel, pentane/ether, 3/1).

Data for anti-28k:

${ }^{1} \mathrm{H}$ NMR: $\quad\left(500 \mathrm{MHz}\right.$, benzene- $\left.d_{6}\right)$

$3.77(\mathrm{dt}, J=9.2,3.2,1 \mathrm{H}, \mathrm{CHOH}) ; 3.44-3.64\left(\mathrm{ABX}, 2 \mathrm{H}, \mathrm{TBSOCH}_{2}\right) ; 3.24(\mathrm{~d}, J=$

$3.0,1 \mathrm{H}, \mathrm{OH}) ; 2.37-2.59\left(\mathrm{~m}, 3 \mathrm{H}, \mathrm{COCH}_{2}, \mathrm{COCH}\right) ; 0.94\left(\mathrm{~s}, 9 \mathrm{H}, \mathrm{C}\left(\mathrm{CH}_{2}\right)_{3}\right) ; 0.93$ $\left(\mathrm{s}, 9 \mathrm{H},\left(\mathrm{CH}_{3}\right)_{3} \mathrm{CSi}\right) ; 0.85\left(\mathrm{~d}, J=6.8,3 \mathrm{H}, \mathrm{CHCH}_{3}\right) ; 0.01\left(\mathrm{~s}, 6 \mathrm{H}, \mathrm{SiCH}_{3}\right)$

${ }^{13} \mathrm{C} \mathrm{NMR}: \quad\left(126 \mathrm{MHz}, \mathrm{CDCl}_{3}\right)$

$216.4(\mathrm{C}(3)) ; 75.2$ (C(5)); 65.9 (C(1)); 49.5 (C(2)); $44.5(\mathrm{C}(4)) ; 34.4(\mathrm{C}(6)) ; 26.0$ $(\mathrm{C}(7)) ; 25.9(\mathrm{C}(10)) ; 18.4(\mathrm{C}(9)) ; 13.0(\mathrm{C}(11)) ; 5.4,-5.4(\mathrm{C}(8))$

IR: (neat) 3524 (m, br); 2957 (s); 2934 (s); 2861 (m); 1706 (m); 1471 (m); 1388 (m); 1364 (m); 1257 (m); 1103 (s); 1007 (m) 
MS: $\quad(\mathrm{FI})$

$303.3\left(0.1, \mathrm{M}^{+}\right) ; 247$ (5); 246 (18); 245 (100); 227 (2); 159 (3)

Opt. rot. : $\quad-12.9\left(\mathrm{CHCl}_{3}, \mathrm{c}=0.65\right)$

TLC: $\quad R_{f}=0.30$ (pentane/ether, $3 / 1, \mathrm{SiO}_{2}$ ) [ $p$-anisaldehyde]

Analysis: $\quad$ Calc for $\mathrm{C}_{16} \mathrm{H}_{34} \mathrm{O}_{3} \mathrm{Si}$ (302.52)

Calc. $\quad$ C $63.52 \%$; $\mathrm{H} 11.33 \%$

Found: $\quad$ C $63.27 \%$; H $11.43 \%$

\section{General Procedure IV: $\quad(1 R, 5 S)-5$-tert-Butyldimethylsilyloxy-1-hydroxy-1-phenyl-3- hexanone (anti-35a)}

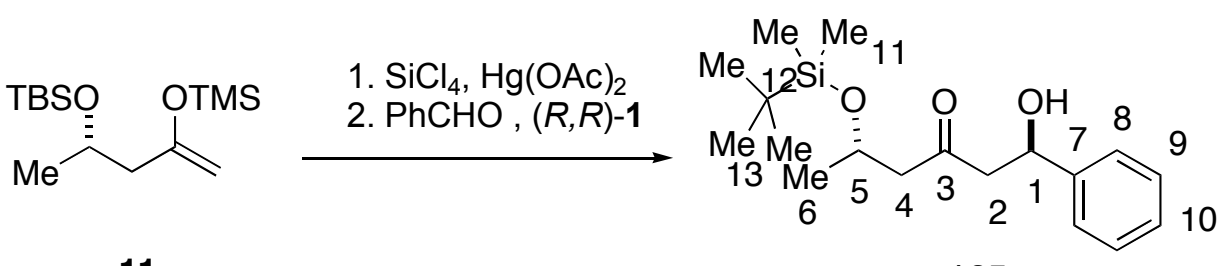

11

anti-35a

To a suspension of mercury(II) acetate $(3.2 \mathrm{mg}, 0.01 \mathrm{mmol}, 0.01$ equiv) in $1 \mathrm{~mL}$ of methylene chloride was added silicon tetrachloride $(0.23 \mathrm{~mL}, 2.0 \mathrm{mmol}, 2.0$ equiv) and the trimethylsilyl enol ether $11(289 \mathrm{mg}, 1.00 \mathrm{mmol})$. The reaction mixture was stirred at rt for 30 min. The excess silicon tetrachloride and the solvent were removed under vacuum. To the residue was added solution of the chiral phosphoramide $(R, R)-\mathbf{1}(36.9 \mathrm{mg}, 0.10 \mathrm{mmol}, 0.10$ equiv) in $2 \mathrm{~mL}$ of methylene chloride via a cannula. The reaction mixture was cooled to $-78{ }^{\circ} \mathrm{C}$ before the addition of benzaldehyde (102 $\mu \mathrm{L}, 1.00 \mathrm{mmol}, 1$ equiv). The reaction mixture was stirred at $-78{ }^{\circ} \mathrm{C}$ for $4 \mathrm{~h}$, and then was quenched by pouring into a vigorously stirring $5 \mathrm{~mL}$ of cold saturated aqueous sodium bicarbonate. The resulting slurry was stirred for $3 \mathrm{~h}$ and was filtered through Celite. The layers were separated and the aqueous layer was extracted with 20 $\mathrm{mL}$ of methylene chloride. The combined organic extracts were washed with $5 \mathrm{~mL}$ of brine, dried over sodium sulfate and concentrated. The crude product was chromatographed (silica gel, pentane/ether, 3/1) to give 35a (198 $\mathrm{mg}, 0.61 \mathrm{mmol}, 61 \%)$ as viscous colorless oil. 


\section{Data for anti-35a:}

${ }^{1}$ H NMR: $\quad\left(500 \mathrm{MHz}, \mathrm{CDCl}_{3}\right)$

7.35 - 7.34 (m, 4 H, HC(8), HC(9)); $7.29-7.26$ (m, $1 \mathrm{H}, \mathrm{HC}(10)) ; 5.15$ (dt, $J=$ 8.6, 3.5, 1H, HC(1)); 4.32 (sext, $J=6.1,1 \mathrm{H}, \mathrm{HC}(5)$ ); 3.36 (d, $J=3.2,1 \mathrm{H}, \mathrm{OH}$ ); $2.92-2.82\left(\mathrm{ABX}, 2 \mathrm{H}, \mathrm{H}_{2} \mathrm{C}(2)\right) ; 2.67-2.41\left(\mathrm{ABX}, 2 \mathrm{H}, \mathrm{H}_{2} \mathrm{C}(4)\right) ; 1.17$ (d, $J=6.2$, $\left.3 \mathrm{H}, \mathrm{H}_{3} \mathrm{C}(6)\right) ; 0.86\left(\mathrm{~s}, 9 \mathrm{H}, \mathrm{H}_{3} \mathrm{C}(13)\right) ; 0.05$ (d, $J=12.4,6 \mathrm{H}, \mathrm{H}_{3} \mathrm{C}(11)$ )

${ }^{13} \mathrm{C}$ NMR: $\quad\left(125 \mathrm{MHz}, \mathrm{CDCl}_{3}\right)$

$210.6(\mathrm{C}(3)) ; 142.7(\mathrm{C}(7)) ; 128.5(\mathrm{C}(9)) ; 127.6(\mathrm{C}(10)) ; 125.6(\mathrm{C}(8)) ; 69.6(\mathrm{C}(5))$; $65.6(\mathrm{C}(1)) ; 53.3(\mathrm{C}(4)) ; 52.9(\mathrm{C}(2)) ; 25.7(\mathrm{C}(13)) ; 24.0(\mathrm{C}(6)) ; 17.9$ (C(12)); $4.5,-5.0(\mathrm{C}(11))$

IR: (neat)

3438 (m, br); 3064 (w); 3032 (w); 2956 (s); 2929 (s); 2895 (m); 2850 (m); 1711 (s); 1495 (w); 1462 (m); 1377 (m); 1255 (m); 1134 (m); 1090 (m); 1066 (s); 1041 (m); 1007 (m)

MS: $\quad(\mathrm{FI})$

$323\left(5, \mathrm{M}^{+}\right) ; 265$ (100); $159(3) ; 106(3)$

Opt. Rot.: $\quad[\alpha]_{\mathrm{D}}^{24}+47.1^{\circ}(\mathrm{EtOH}, \mathrm{c}=0.64)$

TLC: $\quad R f=0.10$ (pentane/ether, 3/1) [silicagel, $p$-anisaldehyde]

SFC: $\quad t_{R}=4.695 \mathrm{~min}(\mathrm{OD}, 150 \mathrm{bar}, 3 \mathrm{~mL} / \mathrm{min}, 2 \% \mathrm{MeOH})$

Analysis: $\quad \mathrm{C}_{18} \mathrm{H}_{30} \mathrm{O}_{3} \mathrm{Si}(322.51)$

Calcd: $\quad$ C, $67.03 \% ; \quad H, 9.38 \%$

Found: $\quad$ C, $66.94 \% ; \quad H, 9.52 \%$

\section{(1S,5S)-5-tert-Butyldimethylsilyloxy-1-hydroxy-1-phenyl-3-hexanone (syn-35a)}

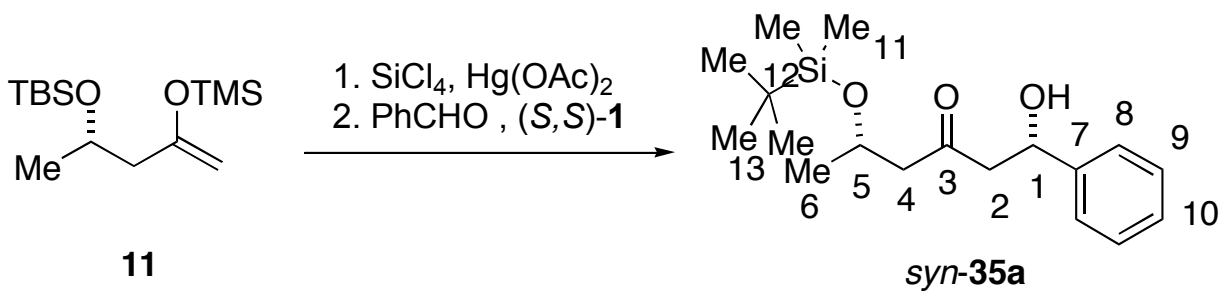

Following General Procedure IV using 11 (289 mg, $1.00 \mathrm{mmol}), \mathrm{SiCl}_{4}(0.23 \mathrm{~mL}, 2.00$ $\mathrm{mmol}), \mathrm{Hg}(\mathrm{OAc})_{2}(3.2 \mathrm{mg}, 0.01 \mathrm{mmol})$, benzaldehyde $(102 \mu \mathrm{L}, 1.00 \mathrm{mmol}),(S, S)-1$ (37 mg, 
$0.10 \mathrm{mmol})$ in $2 \mathrm{~mL}$ of $\mathrm{CH}_{2} \mathrm{Cl}_{2}, 188 \mathrm{mg}(0.58 \mathrm{mmol}, 58 \%)$ of syn-35a was obtained as colorless viscous oil after column chromatography (silica gel, pentane/ether, 3/1).

Data for syn-35a:

${ }^{1} \mathrm{H}$ NMR: $\quad\left(500 \mathrm{MHz}, \mathrm{CDCl}_{3}\right)$

7.36 - 7.34 (m, 4 H, HC(8), HC(9)); 7.29 - 7.26 (m, 1 H, HC(10)); 5.16 - 5.13 (m, 1H, HC(1)); 4.32 (sext, $J=6.1,1 \mathrm{H}, \mathrm{HC}(5)$ ); 3.38 (d, $J=3.2,1 \mathrm{H}, \mathrm{OH}) ; 2.88$ - 2.85 (ABX, 2H, $\left.\mathrm{H}_{2} \mathrm{C}(2)\right) ; 2.68-2.43$ (ABX, 2H, $\left.\mathrm{H}_{2} \mathrm{C}(4)\right) ; 1.17$ (d, $J=6.1,3 \mathrm{H}$, $\left.\mathrm{H}_{3} \mathrm{C}(6)\right) ; 0.86\left(\mathrm{~s}, 9 \mathrm{H}, \mathrm{H}_{3} \mathrm{C}(13)\right) ; 0.06$ (d, $J=10.3,6 \mathrm{H}, \mathrm{H}_{3} \mathrm{C}(11)$ )

${ }^{13} \mathrm{C} \mathrm{NMR}: \quad\left(126 \mathrm{MHz}, \mathrm{CDCl}_{3}\right)$

$210.5(\mathrm{C}(3)) ; 142.7(\mathrm{C}(7)) ; 128.5(\mathrm{C}(9)) ; 127.6(\mathrm{C}(10)) ; 125.6(\mathrm{C}(8)) ; 69.8(\mathrm{C}(5))$; $65.5(\mathrm{C}(1)) ; 53.0(\mathrm{C}(4)) ; 52.8(\mathrm{C}(2)) ; 25.8(\mathrm{C}(13)) ; 24.0(\mathrm{C}(6)) ; 18.0(\mathrm{C}(12))$; $-4.6,-5.0(\mathrm{C}(11))$

IR: (neat)

3441 (m, br); 3064 (w); 3032 (w); 2956 (s); 2930 (s); 2895 (m); 2858 (m); 1710 (m); 1495 (w); 1462 (m); 1376 (m); 1253 (m); 1132 (m); 1090 (m); 1068 (m); $1032(\mathrm{~m}) ; 1007(\mathrm{~m})$

MS: $\quad(\mathrm{FI})$

$323\left(5, \mathrm{M}^{+}\right) ; 265(100) ; 159(3) ; 106(3)$

Opt. Rot.: $\quad[\alpha]_{D}^{24}+1.61(\mathrm{EtOH}, \mathrm{c}=0.95)$

TLC: $\quad R f=0.11$ (pentane/ether, 3/1) [silicagel, $p$-anisaldehyde]

SFC: $\quad t_{R}=4.984 \mathrm{~min}(\mathrm{OD}, 150 \mathrm{bar}, 3 \mathrm{~mL} / \mathrm{min}, 2 \% \mathrm{MeOH})$

Analysis: $\quad \mathrm{C}_{18} \mathrm{H}_{30} \mathrm{O}_{3} \mathrm{Si}(322.51)$

Calcd: $\quad$ C, $67.03 \% ; \quad H, 9.38 \%$

Found: $\quad$ C, $67.00 \% ; \quad H, 9.57 \%$ 


\section{$(1 R, 5 S)-5$-Triisopropylsilyloxy-1-hydroxy-1-phenyl-3-hexanone (anti-37a)}

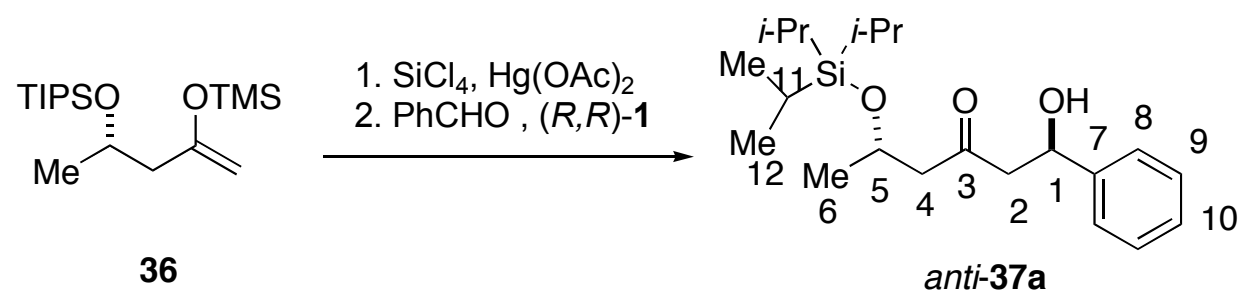

Following General Procedure IV using 36 (331 mg, $1.00 \mathrm{mmol}), \mathrm{SiCl}_{4}(0.23 \mathrm{~mL}, 2.00$ $\mathrm{mmol}), \mathrm{Hg}(\mathrm{OAc})_{2}(3.2 \mathrm{mg}, 0.01 \mathrm{mmol})$, benzaldehyde $(102 \mu \mathrm{L}, 1.00 \mathrm{mmol}),(R, R)-1(37 \mathrm{mg}$, $0.10 \mathrm{mmol})$ in $2 \mathrm{~mL}$ of $\mathrm{CH}_{2} \mathrm{Cl}_{2}, 325 \mathrm{mg}(0.89 \mathrm{mmol}, 89 \%)$ of anti-37a was obtained as colorless viscous oil after column chromatography (silica gel, pentane/ether, 3/1).

Data for anti-37a:

${ }^{1} \mathrm{H} \mathrm{NMR}: \quad\left(500 \mathrm{MHz}, \mathrm{CDCl}_{3}\right)$

7.37 (m, $4 \mathrm{H}, \mathrm{HC}(8), \mathrm{HC}(9)) ; 7.30$ (m, $1 \mathrm{H}, \mathrm{HC}(10)) ; 5.17$ (dt, $J=9.0,3.1,1 \mathrm{H}$, $\mathrm{HC}(1)$ ); 4.47 (sext, $J=6.1,1 \mathrm{H}, \mathrm{HC}(5)) ; 3.35$ (d, $J=3.2,1 \mathrm{H}, \mathrm{OH}) ; 2.96-2.85$ $\left(\mathrm{ABX}, 2 \mathrm{H}, \mathrm{H}_{2} \mathrm{C}(2)\right) ; 2.73-2.52\left(\mathrm{ABX}, 2 \mathrm{H}, \mathrm{H}_{2} \mathrm{C}(4)\right) ; 1.24(\mathrm{~d}, J=6.1,3 \mathrm{H}$, $\left.\mathrm{H}_{3} \mathrm{C}(6)\right) ; 1.07$ (s, 21H, $\left.\mathrm{H}_{3} \mathrm{C}(12), \mathrm{HC}(11)\right)$

${ }^{13} \mathrm{C}$ NMR: $\quad\left(126 \mathrm{MHz}, \mathrm{CDCl}_{3}\right)$

$210.3(\mathrm{C}(3)) ; 142.7(\mathrm{C}(7)) ; 128.5(\mathrm{C}(9)) ; 127.6(\mathrm{C}(10)) ; 125.6(\mathrm{C}(8)) ; 69.7(\mathrm{C}(1))$; 65.4 (C(5)); 53.3 (C(4)); 52.8 (C(2)); 24.1 (C(6)); 18.1 (C(12)); 12.4 (C(11))

IR: (neat)

3441 (m, br); 2961 (s); 2944 (s); 2893 (s); 2867 (s); 1710 (s); 1463 (m); 1375 (m); 1130 (s); 1092 (s); 1066 (s); 1040 (m); 1013 (s)

$\underline{\mathrm{MS}}: \quad(\mathrm{FI})$

$365\left(2, \mathrm{M}^{+}\right) ; 321(100) ; 215(7) ; 144(2) ; 106(3)$

Opt. Rot.: $\quad[\alpha]_{D}^{24}+11.5(\mathrm{EtOH}, \mathrm{c}=0.43)$

TLC: $\quad R f=0.15$ (pentane/ether, 3/1) [silica gel, $p$-anisaldehyde]

SFC: $\quad t_{R}=3.745 \mathrm{~min}(\mathrm{OD}, 150 \mathrm{bar}, 3 \mathrm{~mL} / \mathrm{min}, 5 \% \mathrm{MeOH})$

Analysis: $\quad \mathrm{C}_{21} \mathrm{H}_{36} \mathrm{O}_{3} \mathrm{Si}(364.59)$
Calcd:
C, 69.18\%;
H, $9.95 \%$

Found:

C, 69.04\%;

H, $10.05 \%$ 


\section{(1S,5S)-5-Triisopropylsilyloxy-1-hydroxy-1-phenyl-3-hexanone (syn-37a)}

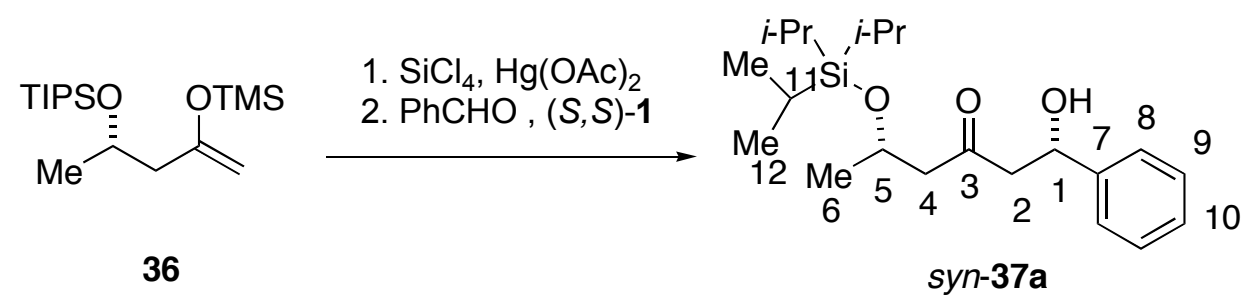

Following General Procedure IV using 36 (331 mg, $1.00 \mathrm{mmol}), \mathrm{SiCl}_{4}(0.23 \mathrm{~mL}, 2.00$ mmol), $\mathrm{Hg}(\mathrm{OAc})_{2}(3.2 \mathrm{mg}, 0.01 \mathrm{mmol})$, benzaldehyde $(102 \mu \mathrm{L}, 1.00 \mathrm{mmol}),(S, S)-1$ (37 mg, $0.10 \mathrm{mmol})$ in $2 \mathrm{~mL}$ of $\mathrm{CH}_{2} \mathrm{Cl}_{2}, 315 \mathrm{mg}(0.86 \mathrm{mmol}, 86 \%)$ of syn-37a was obtained as colorless viscous oil after column chromatography (silica gel, pentane/ether, 3/1).

Data for syn-37a:

${ }^{1} \mathrm{H} \mathrm{NMR}: \quad\left(500 \mathrm{MHz}, \mathrm{CDCl}_{3}\right)$

7.37 (m, 4 H, HC(8), HC(9)); 7.30 (m, 1 H, HC(10)); 5.17 (m, 1H, HC(1)); 4.45 (sext, $J=6.1,1 \mathrm{H}, \mathrm{HC}(5)) ; 3.35$ (d, $J=2.9,1 \mathrm{H}, \mathrm{HO}) ; 2.96-2.85$ (ABX, 2H, $\left.\mathrm{H}_{2} \mathrm{C}(2)\right) ; 2.73-2.59\left(\mathrm{ABX}, 2 \mathrm{H}, \mathrm{H}_{2} \mathrm{C}(4)\right) ; 1.24$ (d, $\left.J=6.1,3 \mathrm{H}, \mathrm{H}_{3} \mathrm{C}(6)\right) ; 1.07$ (s, $\left.21 \mathrm{H}, \mathrm{H}_{3} \mathrm{C}(13), \mathrm{HC}(11)\right)$

${ }^{13} \mathrm{C}$ NMR: $\quad\left(126 \mathrm{MHz}, \mathrm{CDCl}_{3}\right)$

$210.2(\mathrm{C}(3)) ; 142.8(\mathrm{C}(7)) ; 128.5(\mathrm{C}(9)) ; 127.6(\mathrm{C}(10)) ; 125.6(\mathrm{C}(8)) ; 69.7(\mathrm{C}(1))$; 65.3 (C(5)); 53.4 (C(4)); 52.5 (C(2)); 24.0 (C(6)); 18.0 (C(12)); 12.3 (C(11))

IR: (neat)

3438 (m, br); 2961 (s); 2944 (s); 2893 (s); 2867 (s); 1710 (s); 1463 (m); 1375 (m); 1130 (m); 1092 (m); 1066 (m); 1038 (m); 1014 (m)

$\underline{\mathrm{MS}}: \quad(\mathrm{FI})$

$365\left(2, \mathrm{M}^{+}\right) ; 321(100) ; 215(6)$

Opt. Rot.: $\quad[\alpha]_{\mathrm{D}}^{24}-5.84(\mathrm{EtOH}, \mathrm{c}=1.07)$

TLC: $\quad R f=0.14$ (pentane/ether, 3/1) [silica gel, $p$-anisaldehyde]

SFC: $\quad t_{R}=4.163 \mathrm{~min}(\mathrm{OD}, 150 \mathrm{bar}, 3 \mathrm{~mL} / \mathrm{min}, 5 \% \mathrm{MeOH})$

Analysis: $\quad \mathrm{C}_{21} \mathrm{H}_{36} \mathrm{O}_{3} \mathrm{Si}(364.59)$
Calcd:
C, 69.18\%;
H, $9.95 \%$

Found:

C, 69.03\%;

H, $10.20 \%$ 


\section{General Procedure V: Aldol Addition of Trimethylsilyl Enol Ethers: $(1 R, 4 S)-5$-tert-}

Butyldimethylsilyloxy-4-methyl-1-hydroxy-1-phenyl-3-pentanone (syn-28a)

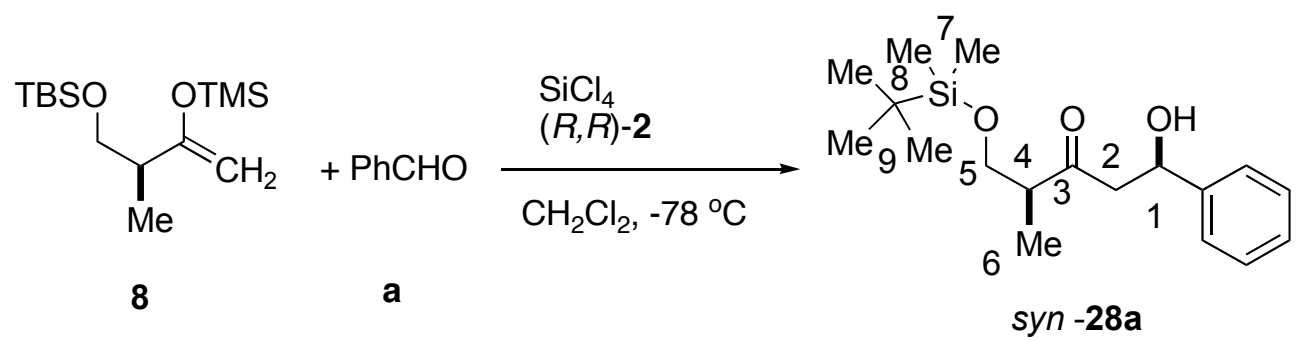

In a $10 \mathrm{~mL}$ Schlenk flask was placed $(R, R)-2(42 \mathrm{mg}, 0.05 \mathrm{mmol}, 0.05$ equiv) in dichloromethane $(1 \mathrm{~mL})$. To the solution was added benzaldehyde $(0.102 \mathrm{~mL}, 1.00 \mathrm{mmol})$, and the solution was cooled to $-78{ }^{\circ} \mathrm{C}$ (bath temperature) using a dry ice/acetone bath. To the solution was added silicon tetrachloride $(0.172 \mathrm{~mL}, 1.50 \mathrm{mmol}, 1.5$ equiv), and the solution was stirred for $5 \mathrm{~min}$. To the solution was added dropwise 8 (346 mg, $1.20 \mathrm{mmol}, 1.2$ equiv), and the reaction mixture was stirred at $-78{ }^{\circ} \mathrm{C}$ (bath temperature) for $4 \mathrm{~h}$. The reaction mixture was quenched by pouring into a vigorously stirring cold $\left(0{ }^{\circ} \mathrm{C}\right)$ saturated aqueous $\mathrm{NaHCO}_{3}$ solution $(15 \mathrm{~mL})$, and the resulting emulsion was stirred for at least $2 \mathrm{~h}$. The emulsion was filtered through a pad of Celite, and the filtrate was transferred into a $60-\mathrm{mL}$ separatory funnel. The biphasic mixture was extracted with dichloromethane $(2 \times 20 \mathrm{~mL})$, and the combined extracts were washed with brine $(10 \mathrm{~mL})$. The solution was dried over $\mathrm{Na}_{2} \mathrm{SO}_{4}$ and concentrated under reduced pressure. The resulting crude oil was chromatographed (silica gel, pentane/ether, 3/1, 30 $\mathrm{mm})$ to afford $294 \mathrm{mg}(0.91 \mathrm{mmol}, 91 \%)$ of $\mathbf{2 8 a}$ as a clear colorless oil.

Data for syn-28a:

${ }^{1} \mathrm{H}$ NMR: $\quad\left(500 \mathrm{MHz}, \mathrm{CDCl}_{3}\right)$

$7.23-7.47(\mathrm{~m}, 5 \mathrm{H}$, aromatic); $5.17(\mathrm{dt}, J=8.8,3.2,1 \mathrm{H}, \mathrm{HC}(1)) ; 3.65-3.76$ $\left(\mathrm{ABX}, 2 \mathrm{H}, \mathrm{C}(5) \mathrm{H}_{2}\right) ; 3.49(\mathrm{~d}, J=2.9,1 \mathrm{H}, \mathrm{OH}) ; 2.86-2.96\left(\mathrm{ABX}, 2 \mathrm{H}, \mathrm{C}(2) H_{2}\right)$; $2.78(\mathrm{~m}, 1 \mathrm{H}, \mathrm{C}(4) H) ; 1.03\left(\mathrm{~d}, J=6.9,3 \mathrm{H}, \mathrm{C}(6) H_{3}\right) ; 0.87\left(\mathrm{~s}, 9 \mathrm{H},\left(\mathrm{C}(9) H_{3}\right)_{3}\right) ; 0.04$ $\left(\mathrm{d}, J=2,6 \mathrm{H}, \mathrm{C}(7) H_{3}\right)$

${ }^{13} \mathrm{C}$ NMR: $\quad\left(125 \mathrm{MHz}, \mathrm{CDCl}_{3}\right)$

214.7 (C(3));142.9, 128.5, 127.5, 125.6 (aromatic); 69.7 (C(1)); $65.6(\mathrm{C}(5)) ; 51.4$ $(\mathrm{C}(2)) ; 49.0(\mathrm{C}(4)) ; 25.8(\mathrm{C}(9)) ; 18.2(\mathrm{C}(8)) ; 12.6(\mathrm{C}(6)) ;-5.6(\mathrm{C}(7))$

TLC: $\quad R_{f}=0.19$ (hexane/EtOAc, $5 / 1, \mathrm{SiO}_{2}$ ) 
SFC: $\quad$ (Chiralpak OD column, $125 \mathrm{bar}, 3.0 \mathrm{~mL} / \mathrm{min}, 2.0 \% \mathrm{MeOH}$ )

$t_{R}(1 R, 4 S)$-syn-28a $4.797 \min (95.9 \%) ;(1 S, 4 S)$-anti-28a $5.271 \min (4.1 \%)$

(1S,4R)-5-tert-Butyldimethylsilyloxy-4-methyl-1-hydroxy-1-phenyl-3-pentanone (anti-28a)

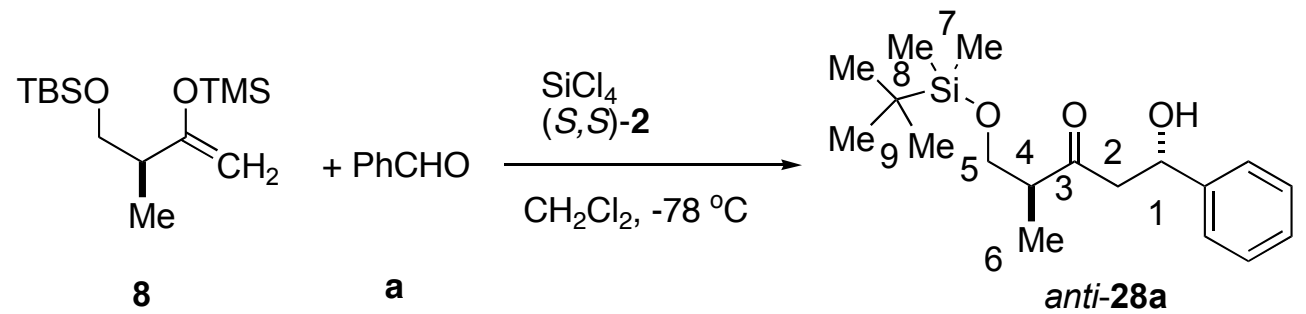

Following General Procedure V using 8 (346 mg, $1.00 \mathrm{mmol}), \mathrm{SiCl}_{4}(0.172 \mathrm{~mL}, 2.00$ mmol), benzaldehyde (102 $\mu \mathrm{L}, 1.00 \mathrm{mmol}),(S, S)-2(42 \mathrm{mg}, 0.05 \mathrm{mmol})$ in $1 \mathrm{~mL}$ of $\mathrm{CH}_{2} \mathrm{Cl}_{2}, 304$ $\mathrm{mg}(0.94 \mathrm{mmol}, 94 \%)$ of anti-28a was obtained as colorless viscous oil after column chromatography (silica gel, pentane/ether, 3/1, $30 \mathrm{~mm}$ ).

Data for anti-28a:

ㅁN NMR: $\quad\left(500 \mathrm{MHz}, \mathrm{CDCl}_{3}\right)$

$7.27-7.38(\mathrm{~m}, 5 \mathrm{H}$, aromatic); $5.14(\mathrm{dt}, J=9.6,2.8,1 \mathrm{H}, \mathrm{HC}(1)) ; 3.67-3.77$ $\left(\mathrm{ABX}, 2 \mathrm{H}, \mathrm{H}_{2} \mathrm{C}(5)\right) ; 3.50(\mathrm{~d}, J=3.0,1 \mathrm{H}, \mathrm{OH}) ; 2.84-2.97\left(\mathrm{ABX}, 2 \mathrm{H}, \mathrm{COC}(2) H_{2}\right)$; $2.78(\mathrm{~m}, 1 \mathrm{H}, \operatorname{COC}(4) H) ; 1.05\left(\mathrm{~d}, J=7.1,3 \mathrm{H}, \mathrm{CHC}(10) H_{3}\right) ; 0.88(\mathrm{~s}, 9 \mathrm{H}$, $\left.\left.\left(\mathrm{C}(13) H_{3}\right)_{3} \mathrm{CSi}\right) ; 0.05\left(\mathrm{~d}, J=2,6 \mathrm{H}, \mathrm{SiC}(11) H_{3}\right)\right)$

${ }^{13} \mathrm{C}$ NMR: $\quad\left(125 \mathrm{MHz}, \mathrm{CDCl}_{3}\right)$

14.7 (C(3)); $142.9(\mathrm{C}(6)), 128.5(\mathrm{C}(8)), 127.5$ (C(9)), 125.6 (C(7)); 70.0 (C(1)); $65.4(\mathrm{C}(5)) ; 51.1(\mathrm{C}(2))$; $48.2(\mathrm{C}(4)) ; 25.8(\mathrm{C}(13))$; $18.2(\mathrm{C}(12)) ; 12.6(\mathrm{C}(10))$; $5.6(\mathrm{C}(11))$

TLC: $\quad R_{f}=0.17$ (hexane/EtOAc, 5/1, $\mathrm{SiO}_{2}$ )

SFC: (Chiralpak OD column, $125 \mathrm{bar}, 3.0 \mathrm{~mL} / \mathrm{min}, 2.0 \% \mathrm{MeOH}$ ) $t_{R} \operatorname{syn-28a} 4.790 \min (2.0 \%) ; t_{R}$ anti-28a $5.203 \min (98.0 \%)$ 
(2S,5R,6E)-1-tert-Butyldimethylsilyloxy-2-methyl-5-hydroxy-7-phenyl-6-hepten-3-one (syn-

28c)

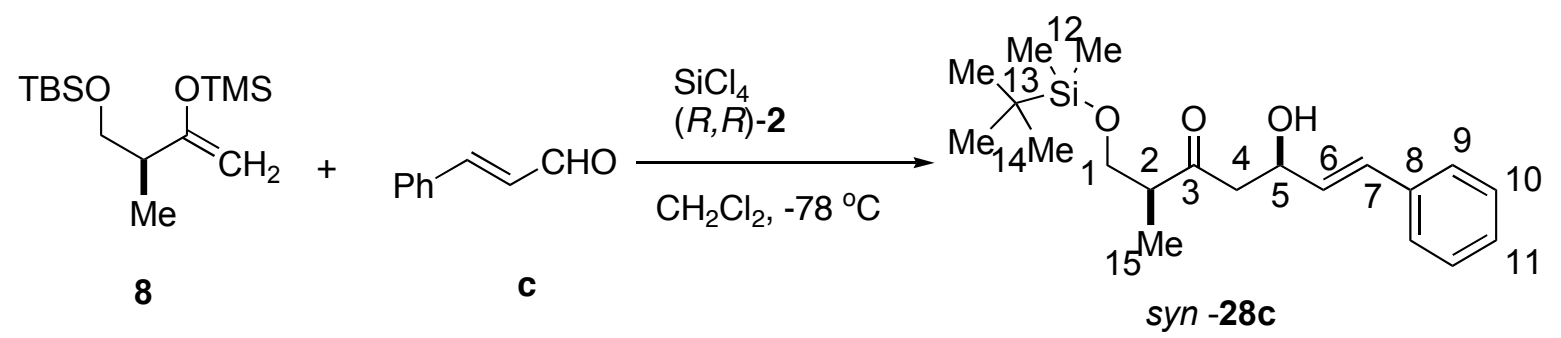

Following General Procedure V using 8 (346 mg, $1.00 \mathrm{mmol}), \mathrm{SiCl}_{4}(0.172 \mathrm{~mL}, 2.00$ mmol), cinnamaldehyde (126 $\mu \mathrm{L}, 1.00 \mathrm{mmol}),(R, R)-2(42 \mathrm{mg}, 0.05 \mathrm{mmol})$ in $1 \mathrm{~mL}$ of $\mathrm{CH}_{2} \mathrm{Cl}_{2}$, $295 \mathrm{mg}(0.85 \mathrm{mmol}, 85 \%)$ of syn-28c was obtained as pale yellow viscous oil after column chromatography (silica gel, pentane/ether, 2/1, $30 \mathrm{~mm}$ ).

Data for syn-28c:

${ }^{1} \mathrm{H}$ NMR: $\quad\left(500 \mathrm{MHz}, \mathrm{CDCl}_{3}\right)$

$7.37(\mathrm{~d}, J=8.6,1 \mathrm{H}$, aromatic); 7.37 (d, $J=5.6,1 \mathrm{H}$, aromatic); 7.30 (t, $J=7.7$, $2 \mathrm{H}$, aromatic); $7.23(\mathrm{t}, J=7.2,1 \mathrm{H}$, aromatic); $6.65(\mathrm{~d}, J=16.2,1 \mathrm{H}, \mathrm{CH}) ; 6.21$ $(\mathrm{dd}, J=16.0,6.0,1 \mathrm{H}, \mathrm{CH}) ; 4.77(\mathrm{~m}, 1 \mathrm{H}, \mathrm{CHOH}) ; 3.66-3.77$ (ABX, $\left.2 \mathrm{H}, \mathrm{TBSOCH}_{2}\right) ; 3.31(\mathrm{~d}, \mathrm{~J}=3.4,1 \mathrm{H}, \mathrm{OH}) ; 2.75-2.90\left(\mathrm{ABX}, 2 \mathrm{H}, \mathrm{COCH}_{2}\right) ; 2.82$ $(\mathrm{m}, 1 \mathrm{H}, \mathrm{COCH}) ; 1.06\left(\mathrm{~d}, J=7.1,3 \mathrm{H}, \mathrm{CHCH}_{3}\right) ; 0.88\left(\mathrm{~s}, 9 \mathrm{H},\left(\mathrm{CH}_{3}\right)_{3} \mathrm{CSi}\right) ; 0.05(\mathrm{~d}, J$ $\left.=5.7,6 \mathrm{H}, \mathrm{SiCH}_{3}\right)$

${ }^{13} \mathrm{C} \mathrm{NMR}: \quad\left(125 \mathrm{MHz}, \mathrm{CDCl}_{3}\right)$

214.4 (C(3)); 136.6 (C(8)), 130.2 (C(11)), 130.0 (C(7)), 128.5 (C(10)); 127.6 $(\mathrm{C}(6)) ; 126.4$ (C(9)); 68.2 (C(1)); $65.5(\mathrm{C}(5)) ; 49.3(\mathrm{C}(2)) ; 49.0(\mathrm{C}(4)) ; 26.0$ $(\mathrm{C}(14)) ; 18.1(\mathrm{C}(13)) ; 12.6(\mathrm{C}(15)) ;-5.6,-5.6(\mathrm{C}(12))$

TLC: $\quad R_{f}=0.12\left(\right.$ hexane/EtOAc, $\left.5 / 1, \mathrm{SiO}_{2}\right)$

SFC: (Chiralpak OD column, $125 \mathrm{bar}, 3.0 \mathrm{~mL} / \mathrm{min}, 7.5 \% \mathrm{MeOH}$ ) $t_{R} \operatorname{syn}-28 \mathrm{c} 3.236 \mathrm{~min}(97.0 \%) ; t_{R}$ anti-28c $3.886 \min (3.0 \%)$ 


\section{(2S,5S,6E)-1-tert-Butyldimethylsilyloxy-2-methyl-5-hydroxy-7-phenyl-6-hepten-3-one (syn-}

28c)

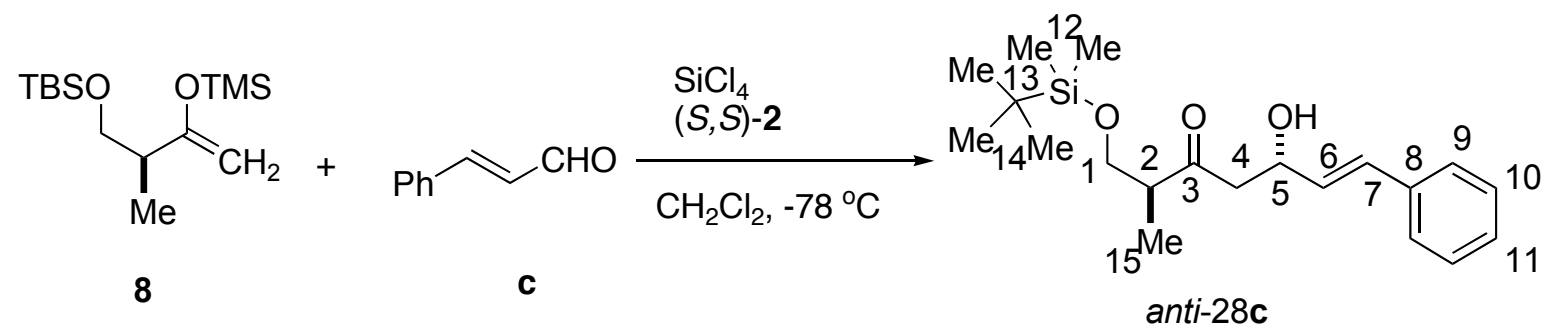

Following General Procedure V using 8 (346 mg, $1.00 \mathrm{mmol}), \mathrm{SiCl}_{4}(0.172 \mathrm{~mL}, 2.00$ mmol), cinnamaldehyde (126 $\mu \mathrm{L}, 1.00 \mathrm{mmol}),(S, S)-2(42 \mathrm{mg}, 0.05 \mathrm{mmol})$ in $1 \mathrm{~mL}$ of $\mathrm{CH}_{2} \mathrm{Cl}_{2}$, $291 \mathrm{mg}(0.83 \mathrm{mmol}, 83 \%)$ of anti-28c was obtained as pale yellow viscous oil after column chromatography (silica gel, pentane/ether, 2/1, $30 \mathrm{~mm}$ ).

Data for anti-28c:

${ }^{1} \mathrm{H}$ NMR: $\quad\left(500 \mathrm{MHz}, \mathrm{CDCl}_{3}\right)$

$7.37(\mathrm{~d}, J=8.6,1.0,1 \mathrm{H}$, aromatic); $7.37(\mathrm{~d}, J=7.7,1.0,1 \mathrm{H}$, aromatic); $7.31(\mathrm{t}, J$ $=7.7,2 \mathrm{H}$, aromatic); $7.23(\mathrm{t}, J=7.2,1 \mathrm{H}$, aromatic $) ; 6.67(\mathrm{dd}, J=16.1,1.4,1 \mathrm{H}$, $\mathrm{CH}) ; 6.22(\mathrm{dd}, J=15.9,6.0,1 \mathrm{H}, \mathrm{CH}) ; 4.75(\mathrm{~m}, 1 \mathrm{H}, \mathrm{CHOH}) ; 3.67$ - $3.78(\mathrm{ABX}$, $\left.2 \mathrm{H}, \mathrm{TBSOCH}_{2}\right) ; 3.34(\mathrm{~d}, \mathrm{~J}=3.4,1 \mathrm{H}, \mathrm{OH}) ; 2.77-2.92\left(\mathrm{ABX}, 2 \mathrm{H}, \mathrm{COCH}_{2}\right) ; 2.84$ $(\mathrm{m}, 1 \mathrm{H}, \mathrm{COCH}) ; 1.07\left(\mathrm{~d}, J=7.1,3 \mathrm{H}, \mathrm{CHCH}_{3}\right) ; 0.88\left(\mathrm{~s}, 9 \mathrm{H},\left(\mathrm{CH}_{3}\right)_{3} \mathrm{CSi}\right) ; 0.05$ (d, $J$ $\left.=3.7,6 \mathrm{H}, \mathrm{SiCH}_{3}\right)$

${ }^{13} \mathrm{C} \mathrm{NMR}: \quad\left(125 \mathrm{MHz}, \mathrm{CDCl}_{3}\right)$

214.3 (C(3)); 136.7 (C(8)), 130.3 (C(11)), 130.2 (C(7)), 128.5 (C(10)); 127.5 $(\mathrm{C}(6))$; 126.5 (C(9)); 68.6 (C(1)); 65.4 (C(5)); 49.3 (C(2)); 48.9 (C(4)); 25.8 $(\mathrm{C}(14)) ; 18.2(\mathrm{C}(13)) ; 12.7$ (C(15)); -5.6 (C(12))

TLC: $\quad R_{f}=0.15$ (hexane/EtOAc, $5 / 1, \mathrm{SiO}_{2}$ )

SFC: (Chiralpak OD column, $125 \mathrm{bar}, 3.0 \mathrm{~mL} / \mathrm{min}, 7.5 \% \mathrm{MeOH}$ ) $t_{R} \operatorname{syn}-28 \mathrm{c} 3.218 \min (2.8 \%) ; t_{R}$ anti-28c $3.854 \min (97.2 \%)$ 
(2S,5R)-1-tert-Butyldimethylsilyloxy-2-methyl-5-hydroxy-5-(1-naphthyl)-3-pentanone (syn28b)
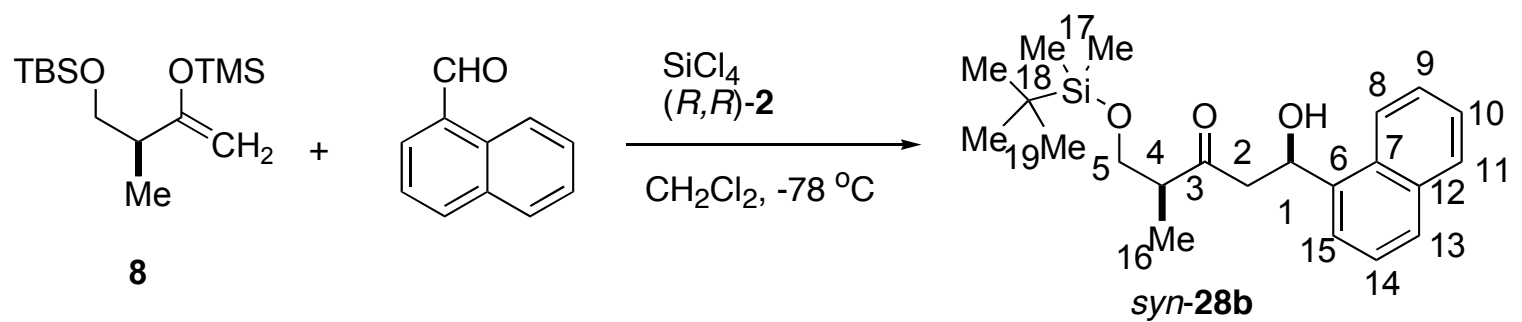

Following General Procedure V using 8 (346 mg, $1.00 \mathrm{mmol}), \mathrm{SiCl}_{4}(0.172 \mathrm{~mL}, 2.00$ mmol), 1-naphthaldehyde (132 $\mu \mathrm{L}, 1.00 \mathrm{mmol}),(R, R)-2(42 \mathrm{mg}, 0.05 \mathrm{mmol})$ in $1 \mathrm{~mL}$ of $\mathrm{CH}_{2} \mathrm{Cl}_{2}$, $316 \mathrm{mg}(0.85 \mathrm{mmol}, 85 \%)$ of syn-28b was obtained as pale yellow viscous oil after column chromatography (silica gel, pentane/ether, 3/1, $30 \mathrm{~mm}$ ).

Data for syn-28b:

${ }^{1} \mathrm{H}$ NMR: $\quad\left(500 \mathrm{MHz}, \mathrm{CDCl}_{3}\right)$

$7.47-8.02(7 \mathrm{H}$, aromatic); $5.97(\mathrm{dt}, J=9.5,2.5,1 \mathrm{H},(\mathrm{C}(1) \mathrm{HOH}) ; 3.68-3.80$ $\left(\mathrm{ABX}, 2 \mathrm{H}, \mathrm{TBSOC}(5) \mathrm{H}_{2}\right) ; 3.58(\mathrm{~d}, J=2.9,1 \mathrm{H}, \mathrm{OH}) ; 2.98-3.13(\mathrm{ABX}, 2 \mathrm{H}$, $\left.\mathrm{COC}(2) H_{2}\right) ; 2.79(\mathrm{~m}, 1 \mathrm{H}, \mathrm{COC}(4) H) ; 1.06\left(\mathrm{~d}, J=7.1,3 \mathrm{H}, \mathrm{CHC}(16) H_{3}\right) ; 0.86(\mathrm{~s}$, 9H, $\left.\left(\mathrm{C}(19) H_{3}\right)_{3} \mathrm{CSi}\right) ; 0.04\left(\mathrm{~d}, J=3.9,6 \mathrm{H}, \mathrm{SiC}(17) H_{3}\right)$

${ }^{13} \mathrm{C} \mathrm{NMR:} \quad\left(125 \mathrm{MHz}, \mathrm{CDCl}_{3}\right)$

214.5 (C(3)); 138.4 (C(6)), 133.7 (C(12)), 129.9 (C(7)), 129.0 (C(11)); 127.9 (C(13)); 126.2 (C(9)); 125.6 (C(10)); 125.5 (C(14)); 123.0 (C(15)); 122.8 (C(8)); 66.6 (C(1)); 65.5 (C(5)); 50.5 (C(2)); 49.2 (C(4)); 25.8 (C(19)); 18.2 (C(18)); 12.6 $(\mathrm{C}(16)) ;-5.6(\mathrm{C}(17))$

TLC: $\quad R_{f}=0.18$ (hexane/EtOAc, $5 / 1, \mathrm{SiO}_{2}$ )

SFC: (Chiralpak OD column, $125 \mathrm{bar}, 3.0 \mathrm{~mL} / \mathrm{min}, 12.5 \% \mathrm{MeOH}$ ) $t_{R} \operatorname{anti-28b} 3.037 \min (5.0 \%) ; t_{R}$ syn-28b $3.786 \min (95.0 \%)$ 
(2S,5S)-1-tert-Butyldimethylsilyloxy-2-methyl-5-hydroxy-5-(1-naphthyl)-3-pentanone (anti28b)
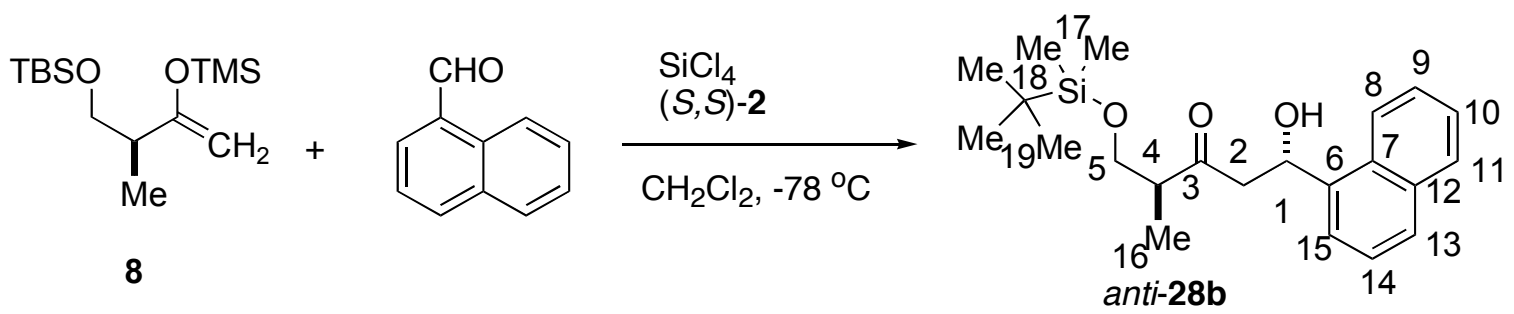

Following General Procedure V using 8 (346 mg, $1.00 \mathrm{mmol}), \mathrm{SiCl}_{4}(0.172 \mathrm{~mL}, 2.00$ mmol), 1-naphthaldehyde (132 $\mu \mathrm{L}, 1.00 \mathrm{mmol}),(S, S)-2(42 \mathrm{mg}, 0.05 \mathrm{mmol})$ in $1 \mathrm{~mL}$ of $\mathrm{CH}_{2} \mathrm{Cl}_{2}$, $308 \mathrm{mg}(0.83 \mathrm{mmol}, 83 \%)$ of anti-28b was obtained as pale yellow viscous oil after column chromatography (silica gel, pentane/ether, 3/1, $30 \mathrm{~mm}$ ).

Data for anti-28b:

${ }^{1} \mathrm{H}$ NMR: $\quad\left(500 \mathrm{MHz}, \mathrm{CDCl}_{3}\right)$

$7.47-8.02(7 \mathrm{H}$, aromatic); $5.98(\mathrm{~m}, J=9.5,1 \mathrm{H}, \mathrm{CHOH}) ; 3.69$ - 3.79 (ABX, 2H,TBSOCH$\left.)_{2}\right) ; 3.68(\mathrm{~d}, J=2.8,1 \mathrm{H}, \mathrm{OH}) ; 3.01-3.09\left(\mathrm{ABX}, 2 \mathrm{H}, \mathrm{COCH}_{2}\right) ; 2.82$ $(\mathrm{m}, 1 \mathrm{H}, \mathrm{COCH}) ; 1.07\left(\mathrm{~d}, J=7.1,3 \mathrm{H}, \mathrm{CHCH}_{3}\right) ; 0.88\left(\mathrm{~s}, 9 \mathrm{H},\left(\mathrm{CH}_{3}\right)_{3} \mathrm{CSi}\right) ; 0.05$ (d, $J$ $\left.=3.9,6 \mathrm{H}, \mathrm{SiCH}_{3}\right)$

${ }^{13} \mathrm{C} \mathrm{NMR}: \quad\left(125 \mathrm{MHz}, \mathrm{CDCl}_{3}\right)$

214.5 (C(3)); 138.4 (C(6)), 133.8 (C(12)), 129.9 (C(7)), 129.0 (C(11)); 128.0 (C(13)); 126.1 (C(9)); 125.6 (C(10)); 125.5 (C(14)); 123.0 (C(15)); 122.8 (C(8)); 66.9 (C(1)); 65.5 (C(5)); 50.2 (C(2)); 49.3 (C(4)); 25.8 (C(19)); 18.2 (C(18)); 12.7 $(\mathrm{C}(16)) ;-5.6(\mathrm{C}(17))$

TLC: $\quad R_{f}=0.19\left(\right.$ hexane/EtOAc, $\left.5 / 1, \mathrm{SiO}_{2}\right)$

SFC: (Chiralpak OD column, $125 \mathrm{bar}, 3.0 \mathrm{~mL} / \mathrm{min}, 12.5 \% \mathrm{MeOH}$ ) $t_{R} \operatorname{anti-28b} 2.947 \min (92.4 \%) ; t_{R} \operatorname{syn}-\mathbf{2 8 b} 3.729 \min (2.2 \%)$ 


\section{(2S,5R)-1-tert-Butyldimethylsilyloxy-2-methyl-5-hydroxy-7-phenyl-6-heptyn-3-one (syn-}

28h)

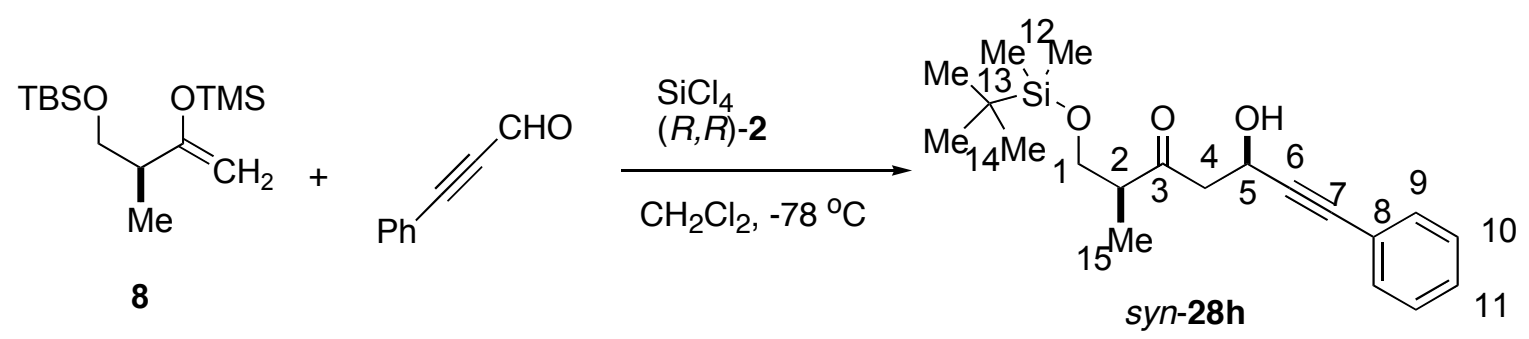

Following General Procedure V using 8 (346 mg, $1.00 \mathrm{mmol}), \mathrm{SiCl}_{4}(0.172 \mathrm{~mL}, 2.00$ mmol), phenylpropargyl aldehyde (122 $\mu \mathrm{L}, 1.00 \mathrm{mmol}),(R, R)-2(42 \mathrm{mg}, 0.05 \mathrm{mmol})$ in $1 \mathrm{~mL}$ of $\mathrm{CH}_{2} \mathrm{Cl}_{2}, 255 \mathrm{mg}(0.74 \mathrm{mmol}, 74 \%)$ of syn-28h was obtained as pale yellow viscous oil after column chromatography (silica gel, pentane/ether, 3/1, $30 \mathrm{~mm}$ ).

Data for syn-28h:

${ }^{1} \mathrm{H}$ NMR: $\quad\left(500 \mathrm{MHz}, \mathrm{CDCl}_{3}\right)$ $7.28-7.43$ (m, 5H, aromatic); $5.01-5.06(\mathrm{~m}, 1 \mathrm{H}, \mathrm{CHOH}) ; 3.67$ - 3.77 (ABX, 2H,TBSOCH$\left.)_{2}\right) ; 3.33(\mathrm{~d}, J=5.1,1 \mathrm{H}, \mathrm{OH}) ; 2.96-3.13\left(\mathrm{ABX}, 2 \mathrm{H}, \mathrm{COCH}_{2}\right) ; 2.82$ $(\mathrm{m}, 1 \mathrm{H}, \mathrm{COCH}) ; 1.08\left(\mathrm{~d}, J=7.1,3 \mathrm{H}, \mathrm{CHCH}_{3}\right) ; 0.88\left(\mathrm{~s}, 9 \mathrm{H},\left(\mathrm{CH}_{3}\right)_{3} \mathrm{CSi}\right) ; 0.06(\mathrm{~d}, J$ $\left.=5.1,6 \mathrm{H}, \mathrm{SiCH}_{3}\right)$

${ }^{13} \mathrm{C} \mathrm{NMR:} \quad\left(125 \mathrm{MHz}, \mathrm{CDCl}_{3}\right)$

213.2 (C(3)); 131.7 (C(9)); 128.4 (C(11)); 128.2 (C(10)); 122.4 (C(8)); 88.5 $(\mathrm{C}(6)) ; 84.6(\mathrm{C}(7)) ; 65.3(\mathrm{C}(5)) ; 58.8(\mathrm{C}(1)) ; 49.4(\mathrm{C}(2)) ; 49.0(\mathrm{C}(4)) ; 25.7$ $(\mathrm{C}(14)) ; 18.1$ (C(13)); $12.4(\mathrm{C}(15)) ;-5.7,-5.7$ (C(12))

TLC: $\quad R_{f}=0.16$ (hexane/EtOAc, $5 / 1, \mathrm{SiO}_{2}$ )

SFC: (Chiralpak OD column, $125 \mathrm{bar}, 3.0 \mathrm{~mL} / \mathrm{min}, 7.5 \% \mathrm{MeOH}$ ) $t_{R} \operatorname{syn}-\mathbf{2 8 h} 3.130 \min (39.6 \%) ; t_{R}$ anti-28h $3.494 \min (60.4 \%)$ 


\section{(2S,5S)-1-tert-Butyldimethylsilyloxy-2-methyl-5-hydroxy-7-phenyl-6-heptyn-3-one (anti-}

28h)

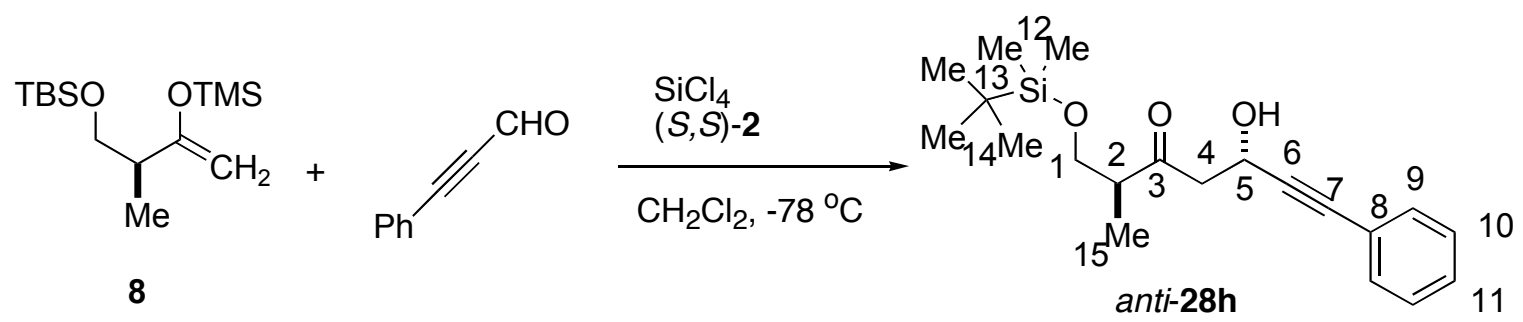

Following General Procedure V using $8(346 \mathrm{mg}, 1.00 \mathrm{mmol}), \mathrm{SiCl}_{4}(0.172 \mathrm{~mL}, 2.00$ mmol), phenylpropargyl aldehyde (122 $\mu \mathrm{L}, 1.00 \mathrm{mmol}),(S, S)-2(42 \mathrm{mg}, 0.05 \mathrm{mmol})$ in $1 \mathrm{~mL}$ of $\mathrm{CH}_{2} \mathrm{Cl}_{2}, 248 \mathrm{mg}(0.72 \mathrm{mmol}, 72 \%)$ of anti-28h was obtained as pale yellow viscous oil after column chromatography (silica gel, pentane/ether, 3/1, $30 \mathrm{~mm}$ ).

Data for anti-28h:

${ }^{1} \mathrm{H}$ NMR: $\quad\left(500 \mathrm{MHz}, \mathrm{CDCl}_{3}\right)$ $7.28-7.43(\mathrm{~m}, 5 \mathrm{H}$, aromatic); $5.01-5.06(\mathrm{~m}, 1 \mathrm{H}, \mathrm{CHOH}) ; 3.67-3.79(\mathrm{ABX}$, 2H,TBSOCH$\left.H_{2}\right) ; 3.29(\mathrm{~d}, J=5.1,1 \mathrm{H}, \mathrm{OH}) ; 2.97-3.13\left(\mathrm{ABX}, 2 \mathrm{H}, \mathrm{COCH}_{2}\right) ; 2.82$ $(\mathrm{m}, 1 \mathrm{H}, \mathrm{COCH}) ; 1.09$ (d, $\left.J=7.1,3 \mathrm{H}, \mathrm{CHCH}_{3}\right) ; 0.88\left(\mathrm{~s}, 9 \mathrm{H},\left(\mathrm{CH}_{3}\right)_{3} \mathrm{CSi}\right) ; 0.05(\mathrm{~d}, J$ $=3.9,6 \mathrm{H}, \mathrm{SiCH}_{3}$ )

${ }^{13} \mathrm{C}$ NMR: $\quad\left(125 \mathrm{MHz}, \mathrm{CDCl}_{3}\right)$

$213.1(\mathrm{C}(3)) ; 131.7(\mathrm{C}(9)) ; 128.4(\mathrm{C}(11)) ; 128.2(\mathrm{C}(10)) ; 122.4(\mathrm{C}(8)) ; 88.5$ $(\mathrm{C}(6)) ; 84.6(\mathrm{C}(7)) ; 65.2(\mathrm{C}(5)) ; 59.0(\mathrm{C}(1)) ; 49.2$ (C(2)); 49.0 (C(4)); 25.8 $(\mathrm{C}(14)) ; 18.1(\mathrm{C}(13)) ; 12.6(\mathrm{C}(15)) ;-5.7(\mathrm{C}(12))$

TLC: $\quad R_{f}=0.14\left(\right.$ hexane/EtOAc, $5 / 1, \mathrm{SiO}_{2}$ )

SFC: (Chiralpak OD column, $125 \mathrm{bar}, 3.0 \mathrm{~mL} / \mathrm{min}, 7.5 \% \mathrm{MeOH}$ ) $t_{R}$ syn-28h $3.097 \min (19.9 \%) ; t_{R}$ anti-28h $3.493 \mathrm{~min}(80.1 \%)$ 
(1R,5S)-5-tert-Butyldimethylsilyloxy-1-hydroxy-1-phenyl-3-hexanone (anti-35a)

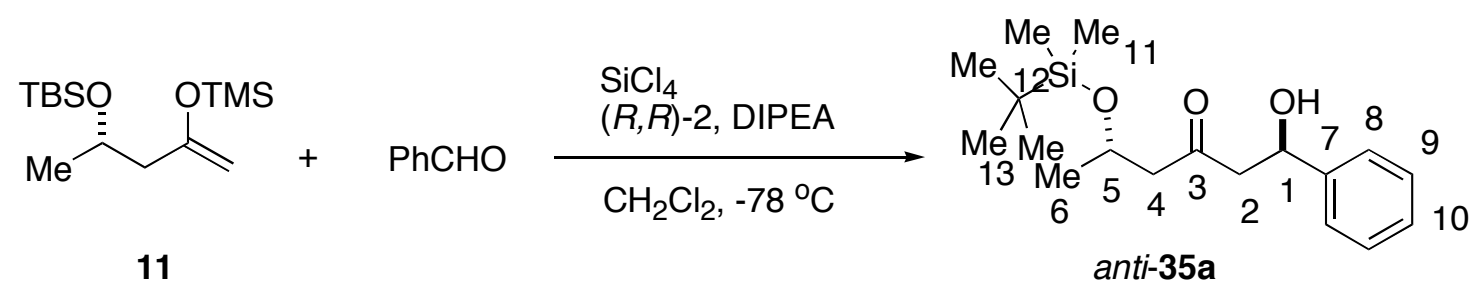

Following General Procedure V using 11 (346 mg, $1.00 \mathrm{mmol}), \mathrm{SiCl}_{4}(0.172 \mathrm{~mL}, 2.00$ mmol), benzaldehyde (102 $\mu \mathrm{L}, \quad 1.00 \mathrm{mmol}), \quad(R, R)-2 \quad(42 \mathrm{mg}, 0.05 \mathrm{mmol})$ and diisopropylethylamine $\left(0.034 \mathrm{~mL}, 0.20 \mathrm{mmol}, 0.2\right.$ equiv) in $2 \mathrm{~mL}$ of $\mathrm{CH}_{2} \mathrm{Cl}_{2}, 298 \mathrm{mg}(0.92$ mmol, 92\%) of anti-35a was obtained as clear colorless oil after column chromatography (silica gel, pentane/ether, 3/1, $30 \mathrm{~mm}$ ).

Data for anti-35a:

${ }^{1} \mathrm{H}$ NMR: $\quad\left(500 \mathrm{MHz}, \mathrm{CDCl}_{3}\right)$

$7.35-7.34$ (m, 4 H, HC(8), HC(9)); $7.29-7.26$ (m, $1 \mathrm{H}, \mathrm{HC}(10)) ; 5.15$ (dt, $J=$ 8.6, 3.5, 1H, HC(1)); 4.32 (sext, $J=6.1,1 \mathrm{H}, \mathrm{HC}(5)) ; 3.36$ (d, $J=3.2,1 \mathrm{H}, \mathrm{OH}$ ); $2.92-2.82\left(\mathrm{ABX}, 2 \mathrm{H}, \mathrm{H}_{2} \mathrm{C}(2)\right) ; 2.67-2.41\left(\mathrm{ABX}, 2 \mathrm{H}, \mathrm{H}_{2} \mathrm{C}(4)\right) ; 1.17$ (d, $J=6.2$, $\left.3 \mathrm{H}, \mathrm{H}_{3} \mathrm{C}(6)\right) ; 0.86\left(\mathrm{~s}, 9 \mathrm{H}, \mathrm{H}_{3} \mathrm{C}(13)\right) ; 0.05$ (d, $J=12.4,6 \mathrm{H}, \mathrm{H}_{3} \mathrm{C}(11)$ )

${ }^{13} \mathrm{C} \mathrm{NMR}: \quad\left(125 \mathrm{MHz}, \mathrm{CDCl}_{3}\right)$

$210.6(\mathrm{C}(3)) ; 142.7(\mathrm{C}(7)) ; 128.5(\mathrm{C}(9)) ; 127.6(\mathrm{C}(10)) ; 125.6(\mathrm{C}(8)) ; 69.6(\mathrm{C}(5))$; $65.6(\mathrm{C}(1)) ; 53.3(\mathrm{C}(4)) ; 52.9(\mathrm{C}(2)) ; 25.7$ (C(13)); 24.0 (C(6)); 17.9 (C(12)); $4.5,-5.0(\mathrm{C}(11))$

TLC: $\quad R_{f}=0.16$ (pentane/ether, $3 / 1, \mathrm{SiO}_{2}$ )

SFC: (Chiralpak OD column, $125 \mathrm{bar}, 3.0 \mathrm{~mL} / \mathrm{min}, 3.0 \% \mathrm{MeOH}$ ) $t_{R} \operatorname{anti-35a} 5.243 \min (97.3 \%) ; t_{R} \operatorname{syn}$-35a $5.614 \min (2.7 \%)$ 


\section{(1S,5S)-5-tert-Butyldimethylsilyloxy-1-hydroxy-1-phenyl-3-hexanone (syn-35a)}

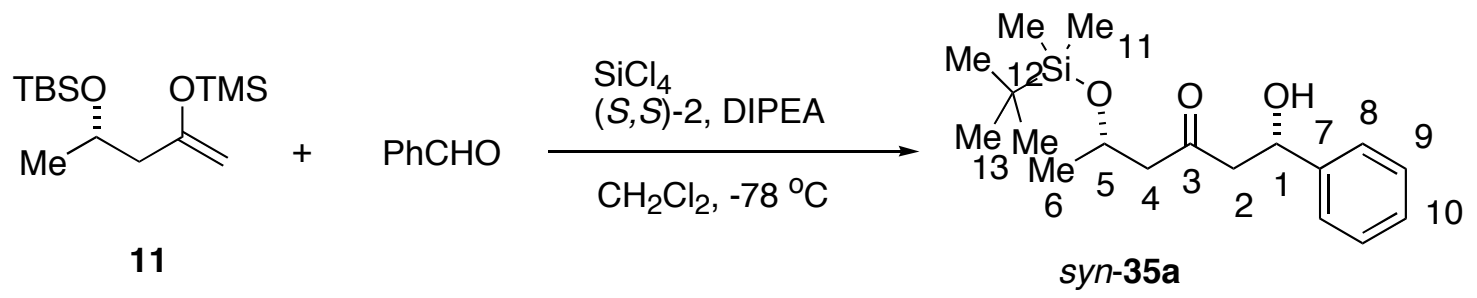

Following General Procedure V using 11 (346 mg, $1.00 \mathrm{mmol}), \mathrm{SiCl}_{4}(0.172 \mathrm{~mL}, 2.00$ mmol), benzaldehyde (102 $\mu \mathrm{L}, 1.00 \mathrm{mmol}), \quad(S, S)-2 \quad(42 \mathrm{mg}, \quad 0.05 \mathrm{mmol})$ and diisopropylethylamine (0.034 mL, $0.20 \mathrm{mmol}, 0.2$ equiv) in $2 \mathrm{~mL}$ of $\mathrm{CH}_{2} \mathrm{Cl}_{2}, 302 \mathrm{mg}(0.94$ mmol, 94\%) of syn-35a was obtained as clear colorless oil after column chromatography (silica gel, pentane/ether, 3/1, $30 \mathrm{~mm}$ ).

Data for syn-35a:

${ }^{1} \mathrm{H}$ NMR: $\quad\left(500 \mathrm{MHz}, \mathrm{CDCl}_{3}\right)$

7.36 - 7.34 (m, 4 H, HC(8), HC(9)); 7.29 - 7.26 (m, 1 H, HC(10)); 5.16 - 5.13 (m, 1H, HC(1)); 4.32 (sext, $J=6.1,1 \mathrm{H}, \mathrm{HC}(5)$ ); 3.38 (d, $J=3.2,1 \mathrm{H}, \mathrm{OH}) ; 2.88$ - 2.85 (ABX, 2H, $\left.\mathrm{H}_{2} \mathrm{C}(2)\right) ; 2.68-2.43$ (ABX, 2H, $\left.\mathrm{H}_{2} \mathrm{C}(4)\right) ; 1.17$ (d, $J=6.1,3 \mathrm{H}$, $\left.\mathrm{H}_{3} \mathrm{C}(6)\right) ; 0.86\left(\mathrm{~s}, 9 \mathrm{H}, \mathrm{H}_{3} \mathrm{C}(13)\right) ; 0.06\left(\mathrm{~d}, J=10.3,6 \mathrm{H}, \mathrm{H}_{3} \mathrm{C}(11)\right)$

${ }^{13} \mathrm{C} \mathrm{NMR}: \quad\left(125 \mathrm{MHz}, \mathrm{CDCl}_{3}\right)$

210.5 (C(3)); 142.7 (C(7)); 128.5 (C(9)); 127.6 (C(10)); 125.6 (C(8)); 69.8 (C(5)); 65.5 (C(1)); 53.0 (C(4)); 52.8 (C(2)); 25.8 (C(13)); 24.0 (C(6)); 17.9 (C(12)); 4.6, -5.0 (C(11))

TLC: $\quad R_{f}=0.19$ (pentane/ether, $3 / 1, \mathrm{SiO}_{2}$ )

SFC: (Chiralpak OD column, $125 \mathrm{bar}, 3.0 \mathrm{~mL} / \mathrm{min}, 3.0 \% \mathrm{MeOH}$ ) $t_{R} \operatorname{anti-35a} 5.276 \min (2.7 \%) ; t_{R} \operatorname{syn}-35 \mathbf{a} 5.577 \min (97.3 \%)$ 
(2S,6R)- 2-(tert-Butyldimethylsilanyloxy)-6-hydroxy-8-phenyl-oct-7-en-4-one (anti-35c)

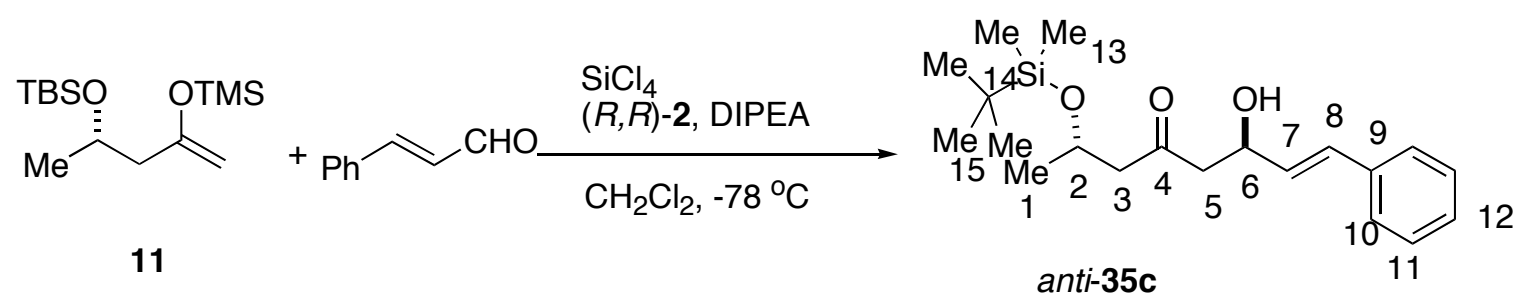

Following General Procedure V using 11 (346 mg, $1.00 \mathrm{mmol}), \mathrm{SiCl}_{4}(0.172 \mathrm{~mL}, 2.00$ mmol), cinnamaldehyde (126 $\mu \mathrm{L}, 1.00 \mathrm{mmol}), \quad(R, R)-2 \quad(42 \mathrm{mg}, 0.05 \mathrm{mmol})$ and diisopropylethylamine $\left(0.034 \mathrm{~mL}, 0.20 \mathrm{mmol}, 0.2\right.$ equiv) in $2 \mathrm{~mL}$ of $\mathrm{CH}_{2} \mathrm{Cl}_{2}, 309 \mathrm{mg}(0.88$ mmol, 88\%) of anti-35c was obtained as pale yellow viscous oil after column chromatography (silica gel, pentane/ether, 2/1, $30 \mathrm{~mm}$ ).

Data for anti-35c:

${ }^{1} \mathrm{H} \mathrm{NMR}: \quad\left(500 \mathrm{MHz}, \mathrm{CDCl}_{3}\right)$

$7.36-7.26$ (m, $5 \mathrm{H}, 2$ x $\mathrm{HC}(7), 2 \times \mathrm{HC}(8), \mathrm{HC}(9)) ; 4.78$ (dd, $J=8.0,4.8,1 \mathrm{H}$, $\mathrm{HC}(1)) ; 3.78$ (ABX, $2 \mathrm{H}, 2$ x HC(5)); 3.09 (d, $J=4.6,1 \mathrm{H}, \mathrm{HO}) ; 3.05$ (pent, $J=$ 7.6, $1 \mathrm{H}, \mathrm{HC}(2)) ; 2.89$ (sextd, $J=5.1,1.2,1 \mathrm{H}, \mathrm{HC}(4)) ; 1.04$ (m, $21 \mathrm{H}, 3$ x $\mathrm{HC}(12), 18 \times \mathrm{HC}(13)) ; 0.97$ (d, $J=7.1,3 \mathrm{H}, 3 \times \mathrm{HC}(10)) ; 0.93$ (d, $J=7.1,3 \mathrm{H}, 3$ $\mathrm{x} \mathrm{HC}(11))$

${ }^{13} \mathrm{C} \mathrm{NMR}: \quad\left(126 \mathrm{MHz}, \mathrm{CDCl}_{3}\right)$

$217.9(\mathrm{C}(3)) ; 142.3(\mathrm{C}(6)) ; 128.3(2 \times \mathrm{C}(7)) ; 127.7(\mathrm{C}(9)) ; 126.5$ (2 x C(8)); 76.5 (C(1)); 65.5 (C(5)); $53.1(\mathrm{C}(2))$; 49.1 (C(4)); 17.9, 17.9 (6 x C(13)); 13.8 (C(10)); $12.8(\mathrm{C}(11)) ; 11.8(3 \times \mathrm{C}(12))$

IR: (neat)

3452 (br, m); 3064 (w); 3032 (w); 2943 (s); 2893 (m); 2868 (s); 1711 (m); 1495 (w); 1462 (m); $1383(\mathrm{~m}) ; 1248$ (m); 1101 (m); 1066 (m); 1011 (m)

MS: $\quad(\mathrm{FI})$

$379\left(3, \mathrm{M}^{+}\right) ; 337$ (7); 336 (25); 335 (100); 272 (4); 229 (4); 58 (7)

Opt. Rot.: $\quad[\alpha]^{24}+29.4^{\circ}(\mathrm{EtOH}, \mathrm{c}=0.7)$

TLC: $\quad R_{f} 0.21$ (silica gel, pentane/ether, 2/1)

SFC: (Chiralpak OD column, $125 \mathrm{bar}, 3.0 \mathrm{~mL} / \mathrm{min}, 5.0 \% \mathrm{MeOH}$ ) $t_{R} \operatorname{syn}-35 \mathrm{c} 7.515 \min (3.2 \%) ; t_{R}$ anti-35c $8.085 \min (96.8 \%)$ 
Analysis: $\quad \mathrm{C}_{22} \mathrm{H}_{38} \mathrm{O}_{3} \mathrm{Si}(348.55)$

$\begin{array}{lll}\text { Calcd: } & \mathrm{C}, 68.92 \% ; & \mathrm{H}, 9.25 \% \\ \text { Found: } & \mathrm{C}, 69.08 \% ; & \mathrm{H}, 9.05 \%\end{array}$

(2S,6S)- 2-(tert-Butyldimethylsilanyloxy)-6-hydroxy-8-phenyl-oct-7-en-4-one (syn-35c)

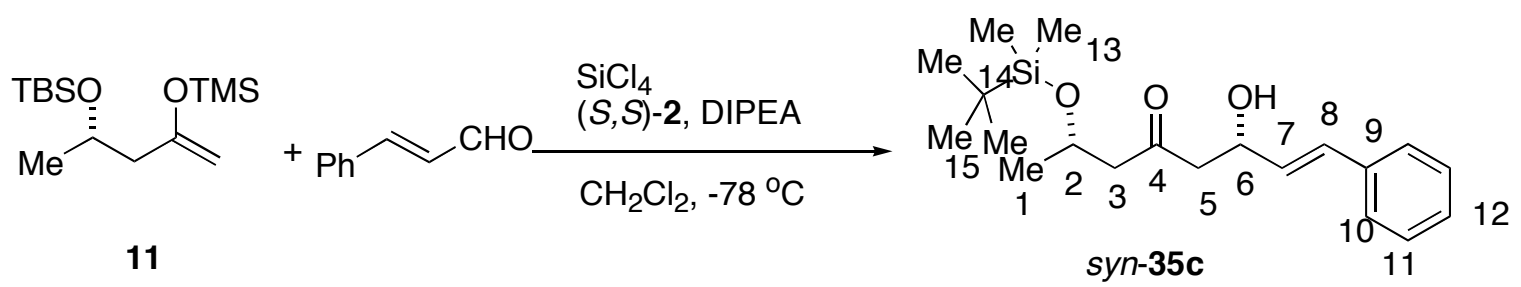

Following General Procedure V using 11 (346 mg, $1.00 \mathrm{mmol}), \mathrm{SiCl}_{4}(0.172 \mathrm{~mL}, 2.00$ mmol), cinnamaldehyde (126 $\mu \mathrm{L}, 1.00 \mathrm{mmol}), \quad(S, S)-2 \quad(42 \mathrm{mg}, 0.05 \mathrm{mmol})$ and diisopropylethylamine $\left(0.034 \mathrm{~mL}, 0.20 \mathrm{mmol}, 0.2\right.$ equiv) in $2 \mathrm{~mL}$ of $\mathrm{CH}_{2} \mathrm{Cl}_{2}, 326 \mathrm{mg}(0.93$ mmol, 93\%) of syn-35c was obtained as pale yellow viscous oil after column chromatography (silica gel, pentane/ether, 2/1, $30 \mathrm{~mm}$ ).

Data for syn-35c:

${ }^{1} \mathrm{H}$ NMR: $\quad\left(500 \mathrm{MHz}, \mathrm{CDCl}_{3}\right)$

$7.37(\mathrm{~d}, J=7.0,2 \mathrm{H}, 2 \times \mathrm{HC}(10)) ; 7.31(\mathrm{t}, J=7.8,2 \mathrm{H}, 2 \times \mathrm{HC}(11)) ; 7.26(\mathrm{t}, J=$ 7.3, $1 \mathrm{H}, \mathrm{HC}(7), \mathrm{HC}(12)) ; 6.63$ (d, $J=16.1,1 \mathrm{H}, \mathrm{HC}(8))$; 6.19 (dd, $J=16.1,6.1$, $1 \mathrm{H}, \mathrm{HC}(7)) ; 4.75$ (m, $1 \mathrm{H}, \mathrm{HC}(6)) ; 4.32$ (sext, $J=6.1,1 \mathrm{H}, \mathrm{HC}(2)) ; 3.17$ (d, $J=$ 3.7, $1 \mathrm{H}, \mathrm{HO}) ; 2.77\left(\mathrm{ABX}, J_{\mathrm{AB}}=17.6,2 \mathrm{H}, 2 \times \mathrm{HC}(5)\right) ; 2.58\left(\mathrm{ABX}, J_{\mathrm{AB}}=15.2,2\right.$ $\mathrm{H}, 2 \times \mathrm{HC}(3)) ; 1.18$ (d, $J=6.1,3 \mathrm{H}, 3 \times \mathrm{HC}(1))$; 0.86 (s, $9 \mathrm{H}, 9 \times \mathrm{HC}(15)) ; 0.07$ (s, 3 H, 3 x HC(13)); 0.05 (s, 3 H, 3 x HC(13'))

${ }^{13} \mathrm{C} \mathrm{NMR}: \quad\left(126 \mathrm{MHz}, \mathrm{CDCl}_{3}\right)$

$210.6(\mathrm{C}(4)) ; 136.5(\mathrm{C}(9)) ; 130.2(\mathrm{C}(12)) ; 130.2(\mathrm{C}(8)) ; 128.5(2 \times \mathrm{C}(11)) ; 127.6$ $(\mathrm{C}(7)) ; 126.4$ (2 x C(10)); 68.3 (C(6)); 65.3 (C(2)); $53.0(\mathrm{C}(3)) ; 50.6(\mathrm{C}(5)) ; 25.7$ $(3 \times \mathrm{C}(15)) ; 23.9(\mathrm{C}(1)) ; 17.8(\mathrm{C}(14)) ;-4.6,-5.0(2 \times \mathrm{C}(13))$

IR: (neat)

3432 (br, m); 3087 (w); 3061 (w); 3028 (w); 2956 (s); 2930 (s); 2895 (m); 2857 (s); 1710 (s); 1638 (w); 1599 (w); 1496 (w); 1472 (m); 1463 (m); 1449 (m); 1376 
(m); 1361 (m); 1256 (s); 1203 (w); 1130 (m); 1083 (m); $1029(\mathrm{~m}) ; 1004(\mathrm{~m})$

$\underline{\mathrm{MS}}: \quad(\mathrm{FI})$

$348\left(100, \mathrm{M}^{+}\right) ; 331$ (7); 291 (44); 244 (9); 216 (5); 159 (11); 146 (18); 132 (16);

$115(5)$

Opt. Rot.: $\quad[\alpha]_{D}^{24}+18.9(\mathrm{EtOH}, \mathrm{c}=1.58)$

TLC: $\quad R_{f} 0.23$ (silica gel, pentane/ether, 2/1)

SFC: $\quad$ (Chiralpak OD column, $125 \mathrm{bar}, 3.0 \mathrm{~mL} / \mathrm{min}, 5.0 \% \mathrm{MeOH}$ )

$t_{R} \operatorname{syn}-35 \mathrm{c} 7.413 \min (99.1 \%) ; t_{R}$ anti-35c $8.069 \min (0.9 \%)$

Analysis: $\quad \mathrm{C}_{22} \mathrm{H}_{38} \mathrm{O}_{3} \mathrm{Si}(348.55)$

Calcd: $\quad$ C, $68.92 \% ; \quad \mathrm{H}, 9.25 \%$

Found: $\quad$ C, $68.73 \% ; \quad H, 9.19 \%$

(1R,5S)- 5-(tert-Butyldimethylsilyloxy)-1-hydroxy-1-naphthalen-1-yl-hexan-3-one (anti-35b)

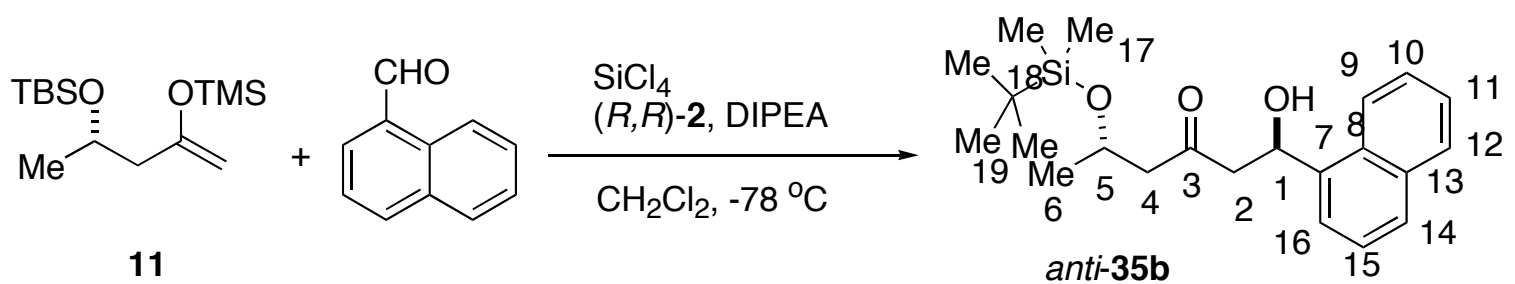

Following General Procedure V using 11 (346 mg, $1.00 \mathrm{mmol}), \mathrm{SiCl}_{4}(0.172 \mathrm{~mL}, 2.00$ mmol), 1-naphthaldehyde (136 $\mu \mathrm{L}, 1.00 \mathrm{mmol}),(R, R)-2 \quad(42 \mathrm{mg}, 0.05 \mathrm{mmol})$ and diisopropylethylamine ( $0.034 \mathrm{~mL}, 0.20 \mathrm{mmol}, 0.2$ equiv) in $2 \mathrm{~mL}$ of $\mathrm{CH}_{2} \mathrm{Cl}_{2}, 276 \mathrm{mg}(0.74$ mmol, 74\%) of anti-35b was obtained as pale yellow viscous oil after column chromatography (silica gel, pentane/ether, 4/1, $30 \mathrm{~mm}$ ).

Data for anti-35b:

${ }^{1} \mathrm{H}$ NMR: $\quad\left(500 \mathrm{MHz}, \mathrm{CDCl}_{3}\right)$

$8.01(\mathrm{dd}, J=7.1,2.5,1 \mathrm{H}, \mathrm{HC}(9)) ; 7.88(\mathrm{dd}, J=6.8,2.7,1 \mathrm{H}, \mathrm{HC}(12)) ; 7.79$ (d, $J$ $=8.3,1 \mathrm{H}, \mathrm{HC}(14)) ; 7.68$ (d, $J=7.1,1 \mathrm{H}, \mathrm{HC}(16)) ; 7.52$ - 7.47 (m, $3 \mathrm{H}, \mathrm{HC}(10)$, $\mathrm{HC}(11), \mathrm{HC}(15)) ; 5.96(\mathrm{td}, J=6.1,3.2,1 \mathrm{H}, \mathrm{HC}(1)) ; 4.37$ (sextd, $J=4.9,1.4,1$ $\mathrm{H}, \mathrm{HC}(5)) ; 3.45(\mathrm{~d}, J=3.2,1 \mathrm{H}, \mathrm{OH}) ; 3.01(\mathrm{~d}, J=6.1,2 \mathrm{H}, 2 \times \mathrm{HC}(2)) ; 2.58$ $\left(\mathrm{ABX}, J_{\mathrm{AB}}=15.1,2 \mathrm{H}, 2 \times \mathrm{HC}(4)\right) ; 1.18(\mathrm{~d}, J=6.1,3 \mathrm{H}, \mathrm{HC}(6)) ; 0.86(\mathrm{~s}, 9 \mathrm{H}, 9$ x HC(19)); 0.08 (s, 3 H, 3 x HC(17)); 0.07 (s, 3 H, 3 x HC(17')) 
${ }^{13} \mathrm{C} \mathrm{NMR}: \quad\left(126 \mathrm{MHz}, \mathrm{CDCl}_{3}\right)$

210.2 (C(3)); 138.2 (C(7)); 133.7 (C(13));129.8 (C(8)); 128.9 (C(12)); 128.0 (C(14)); 126.1 (C(10)); 125.5 (C(11)); 125.5 (C(15)); 123.0 (C(16)); 122.8 (C(9)); $66.5(\mathrm{C}(1)) ; 65.4(\mathrm{C}(5)) ; 52.9(\mathrm{C}(4)) ; 52.2$ (C(2)); 25.8 (3 x C(19)); 23.9 (C(6)); 17.9 (C(18)); -4.5 (C(17)); -4.9 (C(17’))

IR: (neat)

3440 (br, m); 3052 (m); 2956 (s); 2929 (s); 2896(m); 2856 (s); 1713 (s); 1598 (w); 1511 (m); 1472 (m); 1463 (m); 1445 (w); 1375 (s); 1361 (m); 1255 (s); $1213(\mathrm{w})$; 1188 (w); 1159 (m); 1138(m); 1092 (s); 1047 (m); 1019 (m);

MS: $\quad(\mathrm{FI})$

$372\left(15, \mathrm{M}^{+}\right) ; 348$ (100); 331 (7); 315 (5); 303 (2); 291 (38); 268 (6); 243 (10); 216 (4); 177 (2); 159 (4); 146 (19); 115 (4); 89 (5)

Opt. Rot.: $\quad[\alpha]^{24}+84.2(\mathrm{EtOH}, \mathrm{c}=1.6)$

TLC: $\quad R_{f} 0.19$ (silica gel, pentane/ether, 4/1)

SFC: (Chiralpak OD column, $125 \mathrm{bar}, 3.0 \mathrm{~mL} / \mathrm{min}, 15.0 \% \mathrm{MeOH}$ ) $t_{R} \operatorname{syn}-35 \mathbf{b} 2.937 \mathrm{~min}(4.5 \%) ; t_{R}$ anti-35b $3.294 \min (95.5 \%)$

Analysis: $\quad \mathrm{C}_{22} \mathrm{H}_{32} \mathrm{O}_{3} \mathrm{Si}(372.57)$
Calcd:
C, $70.92 \%$;
$\mathrm{H}, 8.66 \%$
Found:
C, $70.71 \%$;
$\mathrm{H}, 8.67 \%$

(1S,5S)- 5-(tert-Butyldimethylsilyloxy)-1-hydroxy-1-naphthalen-1-yl-hexan-3-one (syn-35b)

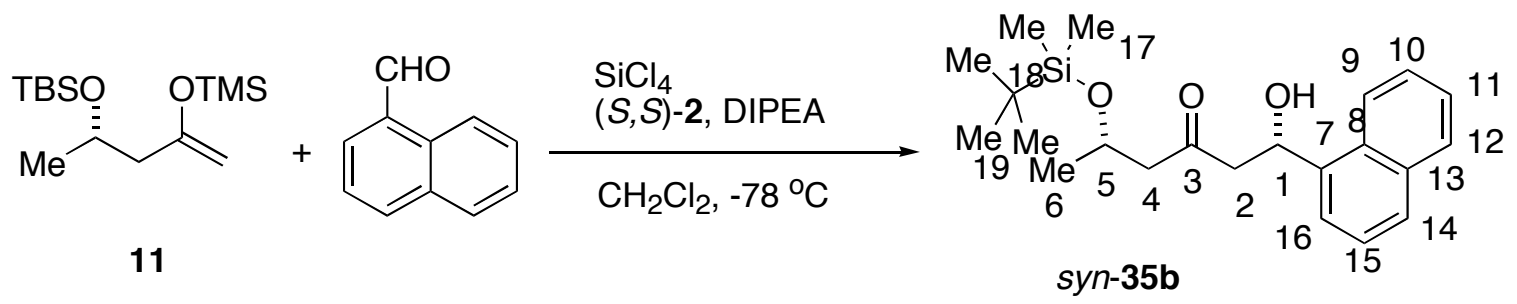

Following General Procedure V using 11 (346 mg, $1.00 \mathrm{mmol}), \mathrm{SiCl}_{4}(0.172 \mathrm{~mL}, 2.00$ mmol), 1-naphthaldehyde (136 $\mu \mathrm{L}, 1.00 \mathrm{mmol}),(S, S)-2 \quad(42 \mathrm{mg}, 0.05 \mathrm{mmol})$ and diisopropylethylamine ( $0.034 \mathrm{~mL}, 0.20 \mathrm{mmol}, 0.2$ equiv) in $2 \mathrm{~mL}$ of $\mathrm{CH}_{2} \mathrm{Cl}_{2}, 294 \mathrm{mg}(0.79$ mmol, $79 \%$ ) of syn-35b was obtained as pale yellow viscous oil after column chromatography 
(silica gel, pentane/ether, 2/1, $30 \mathrm{~mm}$ ).

Data for syn-35b:

$-{ }^{1}$ H NMR: $\quad\left(500 \mathrm{MHz}, \mathrm{CDCl}_{3}\right)$

8.01 (d, $J=8.0,1 \mathrm{H}, \mathrm{HC}(9)) ; 7.88$ (d, $J=8.3,1 \mathrm{H}, \mathrm{HC}(12)) ; 7.79$ (d, $J=8.3,1 \mathrm{H}$, HC(14)); 7.69 (d, J = 7.3, 1 H, HC(16)); 7.53 - 7.47 (m, 3 H, HC(10), HC(11), $\mathrm{HC}(15)) ; 5.93$ (dd, $J=9.2,2.9,1 \mathrm{H}, \mathrm{HC}(1)) ; 4.35$ (sext, $J=6.0,1 \mathrm{H}, \mathrm{HC}(5)$ ); 3.51 $(\mathrm{d}, J=2.7,1 \mathrm{H}, \mathrm{OH}) ; 3.02\left(\mathrm{ABX}, J_{\mathrm{AB}}=18.1,2 \mathrm{H}, 2 \times \mathrm{HC}(2)\right) ; 2.59\left(\mathrm{ABX}, J_{\mathrm{AB}}=\right.$ 14.9, 2 H, 2 x HC(4)); 1.19 (d, $J=6.1,3$ H, HC(6)); 0.85 (s, 9 H, 9 x HC(19)); 0.07 (s, 3 H, 3 x HC(17)); 0.05 (s, 3 H, 3 x HC(17’))

${ }^{13} \mathrm{C} \mathrm{NMR}: \quad\left(126 \mathrm{MHz}, \mathrm{CDCl}_{3}\right)$

$210.5(\mathrm{C}(3)) ; 138.2(\mathrm{C}(7)) ; 133.7(\mathrm{C}(13)) ; 129.8(\mathrm{C}(8)) ; 128.9(\mathrm{C}(12)) ; 127.9$ (C(14)); 126.1 (C(10)); 125.5 (C(11)); 125.4 (C(15)); 123.0 (C(16)); 122.8 (C(9)); 66.6 (C(1)); 65.5 (C(5)); 53.0 (C(4)); 52.1 (C(2)); 25.7 (3 x C(19)); 24.0 (C(6)); $17.9(\mathrm{C}(18)) ;-4.5(\mathrm{C}(17)) ;-5.0\left(\mathrm{C}\left(17^{\prime}\right)\right)$

IR: (neat)

3468 (br, m); 2930 (s); 2857 (s); 1708 (s); 1472 (m); 1376 (m); 1257 (m); 1129 (m); $1095(\mathrm{~m})$

MS: $\quad(\mathrm{FI})$

$372\left(100, \mathrm{M}^{+}\right) ; 348(3) ; 315(7) ; 267(9) ; 156(7)$

Opt. Rot.: $\quad[\alpha]_{D}^{24}-8.9(\mathrm{EtOH}, \mathrm{c}=1.25)$

TLC: $\quad R_{f} 0.39$ (silica gel, pentane/ether, 2/1)

SFC: $\quad$ (Chiralpak OD column, $125 \mathrm{bar}, 3.0 \mathrm{~mL} / \mathrm{min}, 15.0 \% \mathrm{MeOH}$ ) $t_{R} \operatorname{syn}-35 \mathbf{b} 2.856 \min (98.4 \%) ; t_{R}$ anti-35b $3.279 \min (1.6 \%)$

Analysis: $\quad \mathrm{C}_{22} \mathrm{H}_{32} \mathrm{O}_{3} \mathrm{Si}(372.57)$

Calcd: $\quad$ C, $70.92 \% ; \quad H, 8.66 \%$

Found: $\quad \mathrm{C}, 70.52 \% ; \quad \mathrm{H}, 8.68 \%$ 


\section{(2S,6R)- 2-(tert-Butyldimethylsilanyloxy)-6-hydroxy-8-phenyl-oct-7-yn-4-one (anti-35h)}

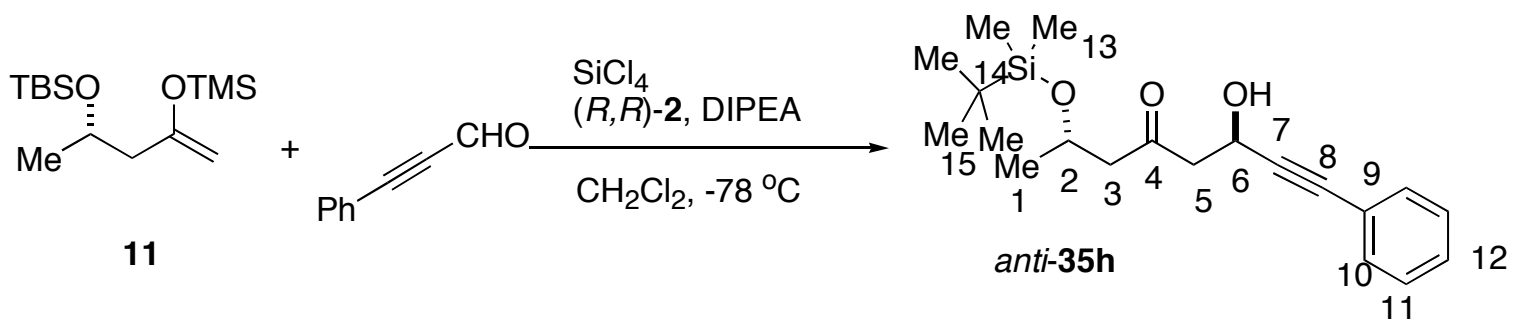

Following General Procedure V using 11 (346 mg, $1.00 \mathrm{mmol}), \mathrm{SiCl}_{4}(0.172 \mathrm{~mL}, 2.00$ mmol), phenylpropargyl aldehyde $(122 \mu \mathrm{L}, 1.00 \mathrm{mmol}),(R, R)-2(42 \mathrm{mg}, 0.05 \mathrm{mmol})$ and diisopropylethylamine ( $0.034 \mathrm{~mL}, 0.20 \mathrm{mmol}, 0.2$ equiv) in $2 \mathrm{~mL}$ of $\mathrm{CH}_{2} \mathrm{Cl}_{2}, 258 \mathrm{mg}(0.74$ mmol, 74\%) of anti-35h was obtained as pale yellow viscous oil after column chromatography (silica gel, pentane/ether, 3/1, $30 \mathrm{~mm}$ ).

Data for anti-35h:

${ }^{1}$ H NMR: $\quad\left(500 \mathrm{MHz}, \mathrm{CDCl}_{3}\right)$

7.43 - 7.28 (m, 5 H, 2 x HC(10), 2 x HC(11), HC(12)); 5.01 (m, $1 \mathrm{H}, \mathrm{HC}(6))$; $4.33(\mathrm{~m}, 1 \mathrm{H}, \mathrm{HC}(2)) ; 3.22(\mathrm{~d}, J=5.1,1 \mathrm{H}, \mathrm{HO}) ; 3.00\left(\mathrm{ABX}, J_{\mathrm{AB}}=18.0,2 \mathrm{H}, 2 \mathrm{x}\right.$ $\mathrm{HC}(5)) ; 2.56\left(\mathrm{ABX}, J_{\mathrm{AB}}=14.8,2 \mathrm{H}, 2 \times \mathrm{HC}(3)\right) ; 1.18(\mathrm{~d}, J=6.2,3 \mathrm{H}, 3 \mathrm{x}$ $\mathrm{HC}(1)) ; 0.87$ (s, 9 H, 9 x HC(15)); 0.07 (s, 3 H, 3 x HC(13)); 0.04 (s, 3 H, 3 x $\left.\mathrm{HC}\left(13^{\prime}\right)\right)$

${ }^{13} \mathrm{C} \mathrm{NMR}: \quad\left(126 \mathrm{MHz}, \mathrm{CDCl}_{3}\right)$

209.3 (C(4)); 131.7 (2 x C(10)); 128.5 (C(12)); 128.2 (2 x C(11)); $122.4(\mathrm{C}(9))$; $88.3(\mathrm{C}(7)) ; 84.7(\mathrm{C}(8)) ; 65.5(\mathrm{C}(2)) ; 58.7(\mathrm{C}(6)) ; 52.9$ (C(3)); $51.0(\mathrm{C}(5)) ; 25.8$ (3 x C(15)); $24.0(\mathrm{C}(1)) ; 17.9(\mathrm{C}(14)) ;-4.5,-5.0$ (2 x C(13))

IR: (neat)

3390 (br, m); 3064 (w); 2955 (s); 2930 (s); 2886 (m); 2857 (s); 2187 (w); 1713 (s); 1652 (s); 1599 (m) 1491 (w); 1472 (m); 1463 (m); 1452 (m); 1444 (m); 1407 (m); 1362 (m); $1315(\mathrm{w}) ; 1255$ (s); 1177 (w); 1072 (m); $1030(\mathrm{~m}) ; 1008(\mathrm{~m})$

MS: $\quad(\mathrm{FI})$

$347\left(32, \mathrm{M}^{+}\right) ; 329$ (5); 289 (100); 159 (3)

Opt. Rot.: $\quad[\alpha]_{\mathrm{D}}^{24}+14.5(\mathrm{EtOH}, \mathrm{c}=1.72)$

TLC: $\quad R_{f} 0.15$ (silica gel, pentane/ether, 3/1) 
SFC: $\quad$ (Chiralpak OD column, $125 \mathrm{bar}, 3.0 \mathrm{~mL} / \mathrm{min}, 5.0 \% \mathrm{MeOH}$ )

$t_{R} \operatorname{syn}$-35h $9.683 \min (44.3 \%) ; t_{R}$ anti-35h $10.472 \min (55.7 \%)$

Analysis: $\quad \mathrm{C}_{20} \mathrm{H}_{30} \mathrm{O}_{3} \mathrm{Si}(346.54)$

Calcd: $\quad$ C, $69.32 \% ; \quad \mathrm{H}, 8.73 \%$

Found: $\quad$ C, $69.00 \% ; \quad H, 8.89 \%$

(2S,6S)- 2-(tert-Butyldimethylsilanyloxy)-6-hydroxy-8-phenyl-oct-7-yn-4-one (syn-35h)

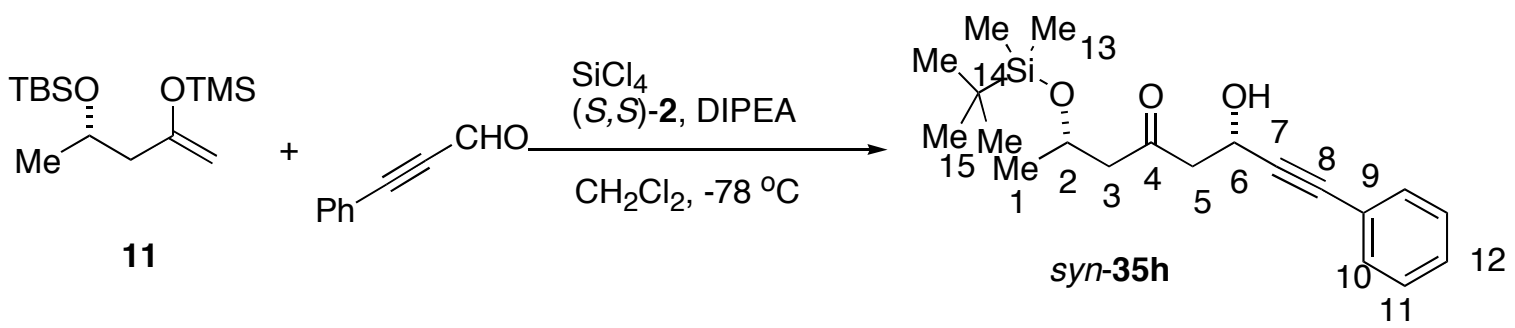

Following General Procedure V using 11 (346 mg, $1.00 \mathrm{mmol}), \mathrm{SiCl}_{4}(0.172 \mathrm{~mL}, 2.00$ mmol), phenylpropargyl aldehyde (122 $\mu \mathrm{L}, 1.00 \mathrm{mmol}),(S, S)-2(42 \mathrm{mg}, 0.05 \mathrm{mmol})$ and diisopropylethylamine $\left(0.034 \mathrm{~mL}, 0.20 \mathrm{mmol}, 0.2\right.$ equiv) in $2 \mathrm{~mL}$ of $\mathrm{CH}_{2} \mathrm{Cl}_{2}, 258 \mathrm{mg}(0.74$ mmol, 74\%) of syn-35h was obtained as pale yellow viscous oil after column chromatography (silica gel, pentane/ether, 3/1, $30 \mathrm{~mm}$ ).

Data for syn-35h:

${ }^{1}$ H NMR: $\quad\left(500 \mathrm{MHz}, \mathrm{CDCl}_{3}\right)$

7.42 - 7.26 (m, 5 H, 2 x HC(10), 2 x HC(11), HC(12)); 5.01 (m, $1 \mathrm{H}, \mathrm{HC}(6))$; $4.31(\mathrm{~m}, 1 \mathrm{H}, \mathrm{HC}(2)) ; 3.33(\mathrm{~d}, J=5.1,1 \mathrm{H}, \mathrm{HO}) ; 3.00\left(\mathrm{ABX}, J_{\mathrm{AB}}=14.7,2 \mathrm{H}, 2 \mathrm{x}\right.$ $\mathrm{HC}(5)) ; 2.60\left(\mathrm{ABX}, J_{\mathrm{AB}}=15.2,2 \mathrm{H}, 2 \times \mathrm{HC}(3)\right) ; 1.17(\mathrm{~d}, J=6.2,3 \mathrm{H}, 3 \times$ $\mathrm{HC}(1))$; 0.86 (s, 9 H, 9 x HC(15)); 0.06 (s, 3 H, 3 x HC(13)); 0.05 (s, 3 H, 3 x $\left.\mathrm{HC}\left(13^{\prime}\right)\right)$

${ }^{13} \mathrm{C} \mathrm{NMR}: \quad\left(126 \mathrm{MHz}, \mathrm{CDCl}_{3}\right)$

208.8 (C(4)); 131.7 (2 x C(10)); 128.4 (C(12)); 128.2 (2 x C(11)); 122.4 (C(9)); $88.4(\mathrm{C}(7)) ; 84.7(\mathrm{C}(8)) ; 65.2(\mathrm{C}(2)) ; 58.7(\mathrm{C}(6)) ; 52.8(\mathrm{C}(3)) ; 51.0(\mathrm{C}(5)) ; 25.7$ $(3 \times \mathrm{C}(15)) ; 23.8(\mathrm{C}(1)) ; 17.8(\mathrm{C}(14)) ;-4.6,-5.1$ (2 x C(13))

IR: (neat)

3391 (br, m); 3064 (w); 2956 (s); 2930 (s); 2886 (m); 2857 (s); 2197 (w); 1713 (s); 1652 (s); 1599 (m) 1491 (w); 1472 (m); 1463 (m); 1452 (m); 1407 (m); 1255 
$(\mathrm{s}) ; 1072(\mathrm{~m})$

MS: $\quad(\mathrm{FI})$

$347\left(15, \mathrm{M}^{+}\right) ; 289$ (100); 271 (4); 159 (4)

Opt. Rot.: $\quad[\alpha]_{D}^{24}+20.5(\mathrm{EtOH}, \mathrm{c}=1.48)$

TLC: $\quad R_{f} 0.12$ (silica gel, pentane/ether, 3/1)

SFC: (Chiralpak OD column, $125 \mathrm{bar}, 3.0 \mathrm{~mL} / \mathrm{min}, 5.0 \% \mathrm{MeOH}$ )

$t_{R} \operatorname{syn-35h} 9.887 \min (46.2 \%) ; t_{R}$ anti-35h $10.671 \min (53.8 \%)$

Analysis: $\quad \mathrm{C}_{20} \mathrm{H}_{30} \mathrm{O}_{3} \mathrm{Si}(346.54)$

Calcd: $\quad$ C, $69.32 \% ; \quad H, 8.73 \%$

Found: $\quad \mathrm{C}, 68.93 \% ; \quad \mathrm{H}, 8.38 \%$

(1R,4S)-1,5-Dihydroxy-4-methyl-1-phenyl-pentan-3-one (31)

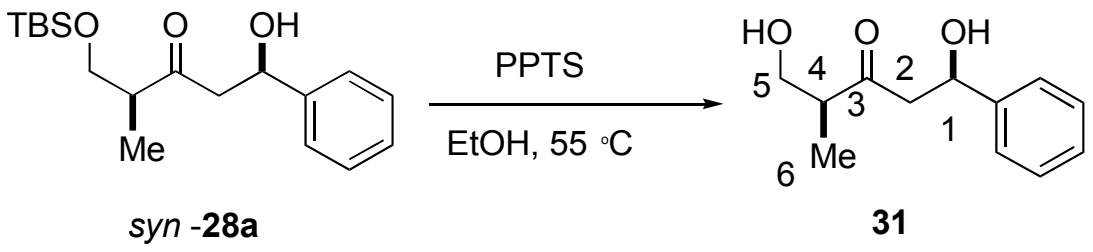

In a 25-mL round-bottom flask were placed syn-28a (83 mg, $0.26 \mathrm{mmol})$, pyridinium $p$ toluenesulfonate $(20 \mathrm{mg}, 0.08 \mathrm{mmol}, 0.33$ equiv) in ethanol $(2 \mathrm{~mL})$. The reaction mixture was heated to $55{ }^{\circ} \mathrm{C}$ (bath temperature) and stirred for $22 \mathrm{~h}$. The reaction mixture was cooled to rt, and the volatiles were removed under reduced pressure. The residue was triturated with ether $(10 \mathrm{~mL})$, and the solution was transferred into a $60-\mathrm{mL}$ separatory funnel. The solition was washed sequentially with brine $(3 \mathrm{~mL})$ and with water $(3 \mathrm{~mL})$, dried over $\mathrm{Na}_{2} \mathrm{SO}_{4}$ and concentrated under reduced pressure. The crude white solid was chromatographed (silica gel, hexanes/EtOAc, 2/1, $10 \mathrm{~mm})$ to afford $43 \mathrm{mg}(0.21 \mathrm{mmol}, 80 \%)$ of $\mathbf{3 1}$ as white needles. The physical properties of the product matched with the literature. ${ }^{8}$ The crystal suitable for X-ray was obtained by recrystallization from acetone/hexane (1/1) by slow evaporation at $\mathrm{rt}$.

Data for 31:

mp: $\quad 88-92{ }^{\circ} \mathrm{C}$ (acetone/hexanes)

${ }^{1} \mathrm{H}$ NMR: $\quad\left(500 \mathrm{MHz}, \mathrm{CDCl}_{3}\right)$

$7.37-7.25\left(\mathrm{~m}, 5 \mathrm{H}\right.$, aromatic); $5.21(\mathrm{dt}, J=9.7,3.0,1 \mathrm{H}, \mathrm{HC}(1)) ; 3.75\left(\mathrm{ABX}, J_{\text {ax }}\right.$ 


$$
\begin{aligned}
& \left.=5.7, J_{\mathrm{bx}}=4.3, J_{\mathrm{ab}}=7.1,2 \mathrm{H}, \mathrm{H}_{2} \mathrm{C}(5)\right) ; 3.19(\mathrm{~d}, J=3.2,1 \mathrm{H}, \mathrm{OH}) ; 2.92\left(\mathrm{ABX}, J_{\mathrm{ax}}\right. \\
& \left.=9.5, J_{\mathrm{bx}}=2.7, J_{\mathrm{ab}}=17.4,2 \mathrm{H}, \mathrm{H}_{2} \mathrm{C}(2)\right) ; 2.81(\mathrm{sext}, J=6.2,1 \mathrm{H}, \mathrm{HC}(4)) ; 2.06(\mathrm{t}, J \\
& =6.2,1 \mathrm{H}, \mathrm{OH}) ; 1.12\left(\mathrm{~d}, J=7.3,3 \mathrm{H}, \mathrm{H}_{3} \mathrm{C}(6)\right)
\end{aligned}
$$

\section{$(1 R, 5 S)-1,5-D i h y d r o x y-1-p h e n y l-h e x a n-3-o n e ~(38)$}<smiles>C[C@H](CC(=O)CC(O)c1ccccc1)OC(C)(C)C</smiles>

anti -35a

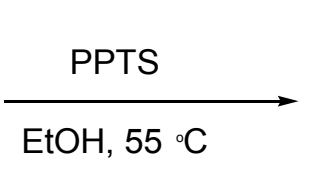

6

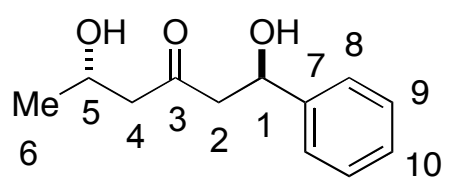

38

In a 25-mL round-bottom flask were placed anti-35a $(242 \mathrm{mg}, 0.75 \mathrm{mmol})$, pyridinium $p$ toluenesulfonate (188 mg, $0.75 \mathrm{mmol}, 1.0$ equiv) in ethanol $(7.5 \mathrm{~mL})$. The reaction mixture was heated to $55{ }^{\circ} \mathrm{C}$ (bath temperature) and stirred for $24 \mathrm{~h}$. The reaction mixture was cooled to rt, and the volatiles were removed under reduced pressure. The residue was triturated with ether $(30 \mathrm{~mL})$, and the solution was transferred into a $60-\mathrm{mL}$ separatory funnel. The solition was washed sequentially with brine $(10 \mathrm{~mL})$ and with water $(10 \mathrm{~mL})$, dried over $\mathrm{Na}_{2} \mathrm{SO}_{4}$ and concentrated under reduced pressure. The crude white solid was chromatographed (silica gel, pentane/ether, 2/1, $20 \mathrm{~mm})$ to afford $94 \mathrm{mg}(0.45 \mathrm{mmol}, 60 \%)$ of $\mathbf{3 8}$ as white needles. The crystal suitable for X-ray was obtained by recrystallization from acetone/hexane (1/1) by slow evaporation at $\mathrm{rt}$.

\section{Data for 38:}

mp: $\quad 58-59{ }^{\circ} \mathrm{C}$ (acetone/hexane)

${ }^{1} \mathrm{H}$ NMR: $\quad\left(500 \mathrm{MHz}, \mathrm{CDCl}_{3}\right.$ )

$7.38-7.27$ (m, $5 \mathrm{H}, 2 \times \mathrm{HC}(8), 2 \times \mathrm{HC}(9), \mathrm{HC}(10)) ; 5.18(\mathrm{dt}, J=9.5,3,1 \mathrm{H}$, $\mathrm{HC}(6)) ; 4.26(\mathrm{~m}, 1 \mathrm{H}, \mathrm{HC}(2)) ; 3.15(\mathrm{~d}, J=3.2,1 \mathrm{H}, \mathrm{HO}) ; 2.85\left(\mathrm{ABX}, J_{\mathrm{AB}}=17.1\right.$, $2 \mathrm{H}, 2$ x $\mathrm{HC}(5)) ; 2.93(\mathrm{~d}, J=3.3,1 \mathrm{H}, \mathrm{HO}) ; 2.60$ (d, $J=5.8,2 \mathrm{H}, 2 \times \mathrm{HC}(3))$; $1.20(\mathrm{~d}, J=6.3,3 \mathrm{H}, 3 \times \mathrm{HC}(1))$

${ }^{13} \mathrm{C} \mathrm{NMR}: \quad\left(126 \mathrm{MHz}, \mathrm{CDCl}_{3}\right)$

211.7 (C(4)); 142.7 (C(7)); 128.6 (2 x C(9)); 127.8 (C(10)); 125.6 (2 x C(8)); 70.0 $(\mathrm{C}(6)) ; 63.9(\mathrm{C}(2)) ; 52.0(\mathrm{C}(3)) ; 51.6(\mathrm{C}(5)) ; 22.5(\mathrm{C}(1))$ 
IR: (neat)

3393 (br, s); 3063 (w); 3031 (w); 2971 (s); 2928 (m); 1707 (s); 1655 (m); 1606

(m); 1577 (w); 1495 (m); 1452 (m); 1376 (m); 1204 (m); 1115 (m); 1049 (m)

MS: $\quad(\mathrm{FI})$

$208\left(100, \mathrm{M}^{+}\right) ; 190(4)$

Opt. Rot.: $\quad[\alpha]_{D}^{24}-11.8(\mathrm{EtOH}, \mathrm{c}=0.06)$

TLC: $\quad R_{f} 0.06$ (silica gel, pentane/ether, 2/1)

Analysis: $\quad \mathrm{C}_{12} \mathrm{H}_{16} \mathrm{O}_{3} \mathrm{Si}(208.25)$

$\begin{array}{lll}\text { Calcd: } & \text { C }, 69.21 \% ; & \text { H }, 7.74 \% \\ \text { Found: } & \text { C }, 69.13 \% ; & \text { H, } 7.77 \%\end{array}$

General Procedure VI: Aldol Addition of 23: (+)-(2S,4R,5S)-5-Hydroxy-4-methyl-5-phenyl2-[((dimethyl)-(1,1-dimethylethyl)silyl)oxy]-3-pentanone (41a) ${ }^{9}$

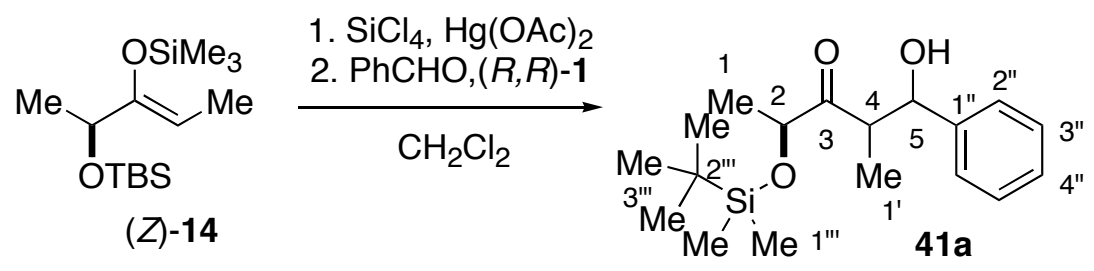

Silyl enol ether $(Z)-\mathbf{1 4}(273 \mathrm{mg}, 1.0 \mathrm{mmol})$ was added quickly to a stirred suspension of silicon tetrachloride $(230 \mu \mathrm{L}, 2.0 \mathrm{mmol}, 2.0$ equiv) and mercuric acetate $(3.2 \mathrm{mg}, 0.01 \mathrm{mmol}$, 0.01 equiv) in $\mathrm{CH}_{2} \mathrm{Cl}_{2}(1.0 \mathrm{~mL})$ at room temperature. After addition, the mixture was stirred at room temperature for $18 \mathrm{~h}$, then the volatile components were removed under reduced pressure $(0.1 \mathrm{mmHg})$ to give a cloudy oil. A solution of $(R, R)-\mathbf{1}(18 \mathrm{mg}, 0.05 \mathrm{mmol}, 0.05$ equiv) in $\mathrm{CH}_{2} \mathrm{Cl}_{2}(2.0 \mathrm{~mL})$ was then added via cannula and the mixture was cooled to $-78{ }^{\circ} \mathrm{C}$. Benzaldehyde $(102 \mu \mathrm{L}, 1.0 \mathrm{mmol})$ was then added dropwise via syringe and the reaction mixture was allowed to stir at $-78{ }^{\circ} \mathrm{C}$ for $10 \mathrm{~h}$. The reaction mixture was then poured into a rapidly stirring sat. aq. $\mathrm{NaHCO}_{3}$ solution $(30 \mathrm{~mL})$ submerged in an ice bath and was allowed to stir at room temperature for $6 \mathrm{~h}$. The heterogeneous mixture was then filtered through Celite, the organic phase was separated and the aqueous phase was extracted with $\mathrm{CH}_{2} \mathrm{Cl}_{2}(3 \times 15 \mathrm{~mL})$. The organic extracts were combined, dried over $\mathrm{Na}_{2} \mathrm{SO}_{4}$, filtered and concentrated to give a crude oil. 
Purification by column chromatography (silica gel, pentane/ $\mathrm{Et}_{2} \mathrm{O}, 6 / 1$ ) afforded $284 \mathrm{mg}(88 \%)$ of $\mathbf{4 1 a}^{10}$ as a clear, colorless oil. The diastereomeric ratio was determined to be (syn,syn)-41a/minor isomers, $95 / 5$ by SFC analysis.

Data for 41a:

${ }^{1}$ H NMR: $\left(\mathrm{CDCl}_{3}, 500 \mathrm{MHz}\right)$

7.38-7.20 (m, $\left.5 \mathrm{H}, 2 \times \mathrm{H}\left(\mathrm{C} 2^{\prime \prime}\right), 2 \times \mathrm{HC}\left(3^{\prime \prime}\right), \mathrm{HC}\left(4^{\prime \prime}\right)\right) ; 5.06(\mathrm{dd}, J=5.0,2.8,1$ $\mathrm{H}, \mathrm{HC}(5)$, syn,syn); 5.01 (dd, $J=5.0,2.8,1 \mathrm{H}, \mathrm{HC}(5)$, anti,syn); 4.77 (dd, $J=$ 8.5, 4.2, $1 \mathrm{H}, \mathrm{HC}(5)$, anti-relative); 4.73 (dd, $J=8.5,4.2,1 \mathrm{H}, \mathrm{HC}(5)$, antirelative); 4.19 (q, $J=6.9,1 \mathrm{H}, \mathrm{HC}(2)) ; 3.37$ (dq, $J=7.2,5.0,1 \mathrm{H}, \mathrm{HC}(4))$; 3.25, (d, $J=2.8,1 \mathrm{H}, \mathrm{OH}) ; 1.27\left(\mathrm{~d}, J=6.9,3 \mathrm{H}, \mathrm{H}_{3} \mathrm{C}(1)\right) ; 1.05(\mathrm{~d}, J=7.2,3$ $\left.\mathrm{H}, \mathrm{H}_{3} \mathrm{C}\left(1^{\prime}\right)\right) ; 0.90\left(\mathrm{~s}, 9 \mathrm{H}, \mathrm{H}_{3} \mathrm{C}\left(3^{\prime \prime \prime}\right)\right) ; 0.08$ (s, $\left.3 \mathrm{H}, \mathrm{H}_{3} \mathrm{C}\left(1^{\prime \prime \prime}\right)\right) ; 0.06$ (s, $3 \mathrm{H}$, $\left.\mathrm{H}_{3} \mathrm{C}\left(1^{\prime \prime \prime}\right)\right)$

${ }^{13} \mathrm{C} \mathrm{NMR}:\left(\mathrm{CDCl}_{3}, 125 \mathrm{MHz}\right)$

218.7 (C(3)); $141.7\left(\mathrm{C}\left(1^{\prime \prime}\right)\right) ; 128.2\left(\mathrm{C}\left(3^{\prime \prime}\right)\right) ; 127.2$ (C(4")); 126.0 (C(2")); 74.6 $(\mathrm{C}(2)) ; 72.8$ (C(5)); 46.9 (C(4)); $25.7\left(\mathrm{C}\left(3^{\prime \prime \prime}\right)\right) ; 21.1$ (C(1)); 18.0 (C(8)); 10.4 $\left(\mathrm{C}\left(1^{\prime}\right)\right) ;-4.7\left(\mathrm{C}\left(1^{\prime \prime \prime}\right)\right) ;-5.0\left(\mathrm{C}\left(1^{\prime \prime \prime}\right)\right)$

TLC: $R_{f} 0.15$ (pentane/Et ${ }_{2} \mathrm{O}, 6 / 1$, anisaldehyde)

SFC: $t_{R}(2 S, 4 R, 5 S)-41 a, 5.1$ min (Daicel Chiralpak AD, 5\% $\mathrm{MeOH}$ in $\mathrm{CO}_{2}, 150$ bar, $40{ }^{\circ} \mathrm{C}, 3.0 \mathrm{~mL} \mathrm{~min}^{-1}$ )

(+)-(2S,4R,5R)-5-Hydroxy-4-methyl-5-(1-naphthyl)-2-[((dimethyl)-(1,1-dimethylethyl)silyl)oxy]-3-pentanone (41b)

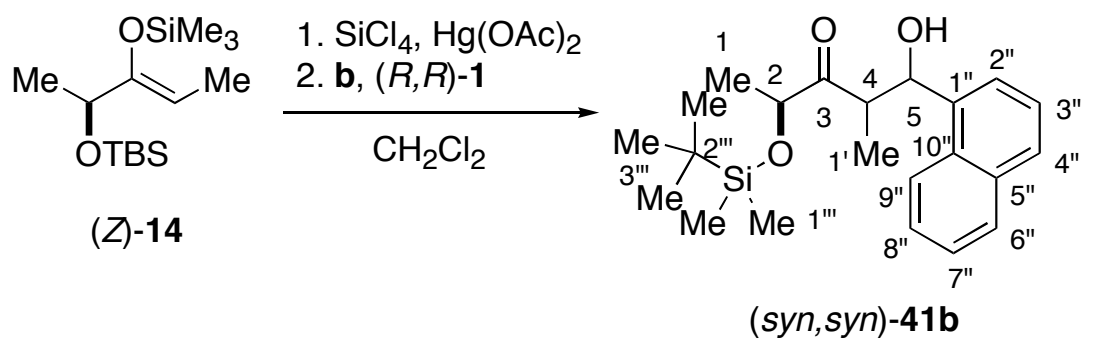

Following General Procedure VI: from silyl enol ether (Z)-14 (273 mg, $1.0 \mathrm{mmol})$, silicon tetrachloride $(230 \mu \mathrm{L}, 2.0 \mathrm{mmol}, 2.0$ equiv) and mercuric acetate $(3.2 \mathrm{mg}, 0.01 \mathrm{mmol}$, 
0.01 equiv) in $\mathrm{CH}_{2} \mathrm{Cl}_{2}(1.0 \mathrm{~mL})$ followed by $(R, R)-\mathbf{1}\left(18 \mathrm{mg}, 0.05 \mathrm{mmol}, 0.05\right.$ equiv) in $\mathrm{CH}_{2} \mathrm{Cl}_{2}$ $(2.0 \mathrm{~mL})$ and 1-naphthaldehyde $(135 \mu \mathrm{L}, 1.0 \mathrm{mmol})$ was obtained after chromatography (silica gel, pentane/ $\left.\mathrm{Et}_{2} \mathrm{O}, 6 / 1\right), 270 \mathrm{mg}(72 \%)$ of $\mathbf{4 1 b}$ as a clear, colorless oil. The diastereomeric ratio was determined to be (syn,syn)-41b/ minor isomers, 98/1/1 by SFC analysis.

Data for 41b:

${ }^{1} \mathrm{H}$ NMR: $\quad\left(\mathrm{CDCl}_{3}, 500 \mathrm{MHz}\right)$

7.90-7.44 (m, 7 H, HC(2"), HC(3"), HC(4"), HC(6"), HC(7"), HC(8"), HC(9")); 5.88 (br s, $1 \mathrm{H}, \mathrm{HC}(5))$ ) 4.27 (q, $J=6.9,1 \mathrm{H}, \mathrm{HC}(2)) ; 3.70$ (d, $J=1.9,1 \mathrm{H}, \mathrm{OH})$; 3.59, (dq, $J=7.3,2.8,1 \mathrm{H}, \mathrm{HC}(4)) ; 1.35$ (d, $\left.J=6.9,3 \mathrm{H}, \mathrm{H}_{3} \mathrm{C}(1)\right) ; 1.05$ (d, $J=$ 7.3, $\left.3 \mathrm{H}, \mathrm{H}_{3} \mathrm{C}\left(1^{\prime}\right)\right)$; 0.86 (s, $\left.9 \mathrm{H}, \mathrm{H}_{3} \mathrm{C}\left(3^{\prime \prime \prime}\right)\right)$; 0.10 (d, J = 1.9, $\left.6 \mathrm{H}, \mathrm{H}_{3} \mathrm{C}\left(1^{\prime \prime \prime}\right)\right)$

${ }^{13} \mathrm{C} \mathrm{NMR}: \quad\left(\mathrm{CDCl}_{3}, 125 \mathrm{MHz}\right)$

$219.5(\mathrm{C}(3)) ; 136.5\left(\mathrm{C}\left(1^{\prime \prime}\right)\right) ; 133.7\left(\mathrm{C}\left(5^{\prime \prime}\right)\right) ; 129.7$ (C(10")); 129.1 (CAr); 127.7 (CAr); 126.0 (CAr); 125.4 (CAr); 125.3 (CAr); 124.4 (CAr); 122.6 (CAr); 74.8 $(\mathrm{C}(2)) ; 69.4$ (C(5)); 45.2 (C(4)); 25.7 (C(3"')); 21.4 (C(1)); 18.1 (C(2"')); 10.2 $\left(\mathrm{C}\left(1^{\prime}\right)\right) ;-4.8\left(\mathrm{C}\left(1^{\prime \prime \prime}\right)\right) ;-5.0\left(\mathrm{C}\left(1^{\prime \prime \prime}\right)\right)$

MS: (FI)

$372\left(\mathrm{M}^{+}, 100\right), 315(6), 267$ (12), $216(5), 156(16)$

IR: (neat) 3502 (br), 2931 (m), 2958 (m), 1699 (m), 1255 (m), 1128 (m), 837 (s), 777 (s)

TLC: $\quad R_{f} 0.19$ (pentane/ $\mathrm{Et}_{2} \mathrm{O}, 6 / 1$, anisaldehyde)

Opt. Rot.: $\quad[\alpha]_{\mathrm{D}}^{23}+53.1^{\circ}\left(c=1.00, \mathrm{CHCl}_{3}\right)$

SFC: $\quad t_{R}(2 S, 4 R, 5 R)-\mathbf{4 1 b}, 2.8 \mathrm{~min}$ (Daicel Chiralpak AS, 4\% $\mathrm{MeOH}$ in $\mathrm{CO}_{2}, 150$ bar, 40 ${ }^{\circ} \mathrm{C}, 3.0 \mathrm{~mL} \min ^{-1}$ )

Analysis: $\quad \mathrm{C}_{22} \mathrm{H}_{32} \mathrm{O}_{3} \mathrm{Si}(372.58)$

Calculated: $\quad$ C, $70.92 ; \quad \mathrm{H}, 8.66 \%$

Found: $\quad$ C, $70.78 ; \quad H, 8.42 \%$ 
(-)-(2S,4R,5S)-(E)-5-Hydroxy-4-methyl-7-phenyl-2-[((dimethyl)-(1,1-dimethylethyl)silyl)oxy]-6-hepten-3-one (41c)

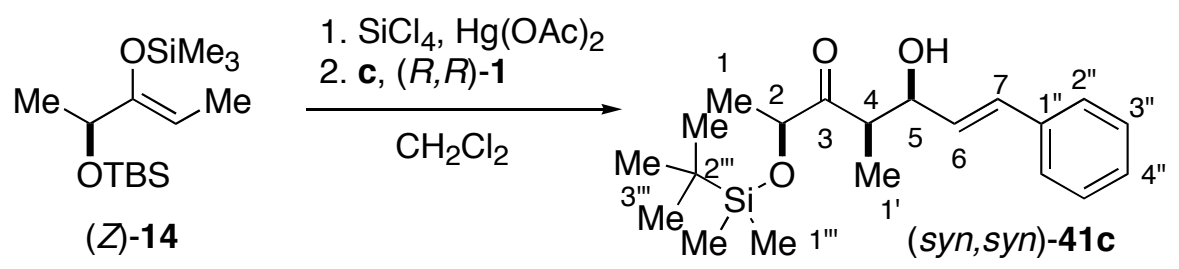

Following General Procedure VI: from silyl enol ether (Z)-14 (273 mg, $1.0 \mathrm{mmol})$, silicon tetrachloride ( $230 \mu \mathrm{L}, 2.0 \mathrm{mmol}, 2.0$ equiv) and mercuric acetate $(3.2 \mathrm{mg}, 0.01 \mathrm{mmol}$, 0.01 equiv) in $\mathrm{CH}_{2} \mathrm{Cl}_{2}(1.0 \mathrm{~mL})$ followed by $(R, R)-1$ (18 mg, $0.05 \mathrm{mmol}, 0.05$ equiv) in $\mathrm{CH}_{2} \mathrm{Cl}_{2}$ $(2.0 \mathrm{~mL})$ and $(E)$-cinnamaldehyde $(130 \mu \mathrm{L}, 1.0 \mathrm{mmol})$ was obtained after chromatography (silica gel, pentane/Et $2 \mathrm{O}, 6 / 1), 283 \mathrm{mg}(81 \%)$ of 41c as a clear, colorless oil. The diastereomeric ratio was determined to be (syn,syn)-41c/minor isomers, 93/5/2 by SFC analysis.

Data for 41c:

${ }^{1} \mathrm{H}$ NMR: $\quad\left(\mathrm{CDCl}_{3}, 500 \mathrm{MHz}\right)$

7.40-7.22 (m, $\left.5 \mathrm{H}, 2 \times \mathrm{H}\left(\mathrm{C}^{\prime \prime}\right), 2 \times \mathrm{HC}\left(3^{\prime \prime}\right), \mathrm{HC}\left(4^{\prime \prime}\right)\right) ; 6.66(\mathrm{dd}, J=15.9,1.5,1 \mathrm{H}$, $\mathrm{HC}(7)) ; 6.14(\mathrm{dd}, J=16.1,5.9,1 \mathrm{H}, \mathrm{HC}(6)) ; 4.61$ (m, $1 \mathrm{H}, \mathrm{HC}(5)) ; 4.25$ (q, $J=$ 6.9, $1 \mathrm{H}, \mathrm{HC}(2)) ; 3.31$ (dq, $J=7.1,3.4,1 \mathrm{H}, \mathrm{HC}(4))$; 3.02, (d, $J=2.8,1 \mathrm{H}, \mathrm{OH}$ ); $1.35\left(\mathrm{~d}, J=6.9,3 \mathrm{H}, \mathrm{H}_{3} \mathrm{C}(1)\right) ; 1.19$ (d, $\left.J=7.1,3 \mathrm{H}, \mathrm{H}_{3} \mathrm{C}\left(1^{\prime}\right)\right) ; 0.93$ (s, $9 \mathrm{H}$, $\left.\mathrm{H}_{3} \mathrm{C}\left(3^{\prime \prime \prime}\right)\right) ; 0.10\left(\mathrm{~d}, J=1.5,6 \mathrm{H}, \mathrm{H}_{3} \mathrm{C}\left(1^{\prime \prime \prime}\right)\right)$

${ }^{13} \mathrm{C} \mathrm{NMR}:\left(\mathrm{CDCl}_{3}, 125 \mathrm{MHz}\right)$

218.2 (C(3)); $136.7\left(\mathrm{C}\left(1^{\prime \prime}\right)\right) ; 131.0\left(\mathrm{C}\left(3^{\prime \prime}\right)\right) ; 129.0\left(\mathrm{C}\left(4^{\prime \prime}\right)\right) ; 128.6(\mathrm{C}(6)) ; 127.6$ $(\mathrm{C}(7)) ; 126.5\left(\mathrm{C}\left(4^{\prime \prime}\right)\right) ; 74.6(\mathrm{C}(2)) ; 71.9$ (C(5)); 45.1 (C(4)); 25.7 (C(3"')); 21.2 $(\mathrm{C}(1)) ; 18.0(\mathrm{C}(8)) ; 10.8\left(\mathrm{C}\left(1^{\prime}\right)\right) ;-4.6\left(\mathrm{C}\left(1^{\prime \prime \prime}\right)\right) ;-5.0\left(\mathrm{C}\left(1^{\prime \prime \prime}\right)\right)$

MS: $\quad(\mathrm{FI})$

$348\left(\mathrm{M}^{+}, 100\right), 291$ (12), 244 (5), $216(6), 159$ (13), 132 (19)

IR: (neat)

3467 (br), 2931 (s), 2858 (m), 1712 (m), 1462 (m), 1365 (m), 1255 (m), 1124 (m), $835(\mathrm{~s}), 779(\mathrm{~s})$

TLC: $\quad R_{f} 0.13$ (pentane/ $\mathrm{Et}_{2} \mathrm{O}, 6 / 1$, anisaldehyde) 
Opt. Rot. : $\quad[\alpha]_{\mathrm{D}}^{23}-17.8^{\circ}\left(c=1.00, \mathrm{CHCl}_{3}\right)$

SFC: $\quad t_{R}(2 S, 4 R, 5 S)-41 c, 5.7$ min (Daicel Chiralpak AD, $1 \% \mathrm{MeOH}$ in $\mathrm{CO}_{2}, 150$ bar, 40 $\left.{ }^{\circ} \mathrm{C}, 3.0 \mathrm{~mL} \min ^{-1}\right)$

Analysis: $\quad \mathrm{C}_{20} \mathrm{H}_{32} \mathrm{O}_{3} \mathrm{Si}(348.56)$

Calculated: $\quad$ C, 68.92; $\quad \mathrm{H}, 9.25 \%$

Found: $\quad$ C, $68.75 ; \quad$ H, $9.20 \%$

(-)-(2S,4R,5S)-(E)-5-Hydroxy-4-methyl-2-[((dimethyl)-(1,1-dimethylethyl)silyl)oxy]-6-octen3-one (syn,syn-41e)

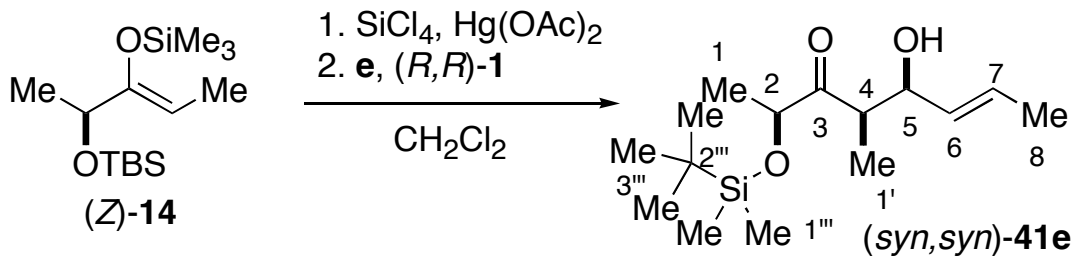

Following General Procedure VI: from silyl enol ether (Z)-14 (273 mg, $1.0 \mathrm{mmol})$, silicon tetrachloride $(230 \mu \mathrm{L}, 2.0 \mathrm{mmol}, 2.0$ equiv) and mercuric acetate $(3.2 \mathrm{mg}, 0.01 \mathrm{mmol}$, 0.01 equiv) in $\mathrm{CH}_{2} \mathrm{Cl}_{2}(1.0 \mathrm{~mL})$ followed by $(R, R)-4\left(18 \mathrm{mg}, 0.05 \mathrm{mmol}, 0.05\right.$ equiv) in $\mathrm{CH}_{2} \mathrm{Cl}_{2}$ $(2.0 \mathrm{~mL})$ and $(E)$-crotanaldehyde $(83 \mu \mathrm{L}, 1.0 \mathrm{mmol})$ was obtained after chromatography (silica gel, pentane/ $\left.\mathrm{Et}_{2} \mathrm{O}, 6 / 1\right), 244 \mathrm{mg}(85 \%)$ of 47 as a clear, colorless oil. The diastereomeric ratio was determined to be (syn,syn)-47/minor isomers, 93/4/3 by SFC analysis.

Data for 47:

${ }^{1} \mathrm{H}$ NMR: $\quad\left(\mathrm{CDCl}_{3}, 500 \mathrm{MHz}\right)$

$5.71(\mathrm{dq}, J=15.2,6.4,1 \mathrm{H}, \mathrm{HC}(7)) ; 5.44(\mathrm{ddd}, J=15.4,6.4,1.7,1 \mathrm{H}, \mathrm{HC}(6))$;

4.33 (br s, $1 \mathrm{H}, \mathrm{HC}(5)$ ); 4.22 (q, $J=7.1,1 \mathrm{H}, \mathrm{HC}(2)) ; 3.19$ (dq, $J=7.1,3.9,1 \mathrm{H}$, $\mathrm{HC}(4)) ; 2.73,(\mathrm{~d}, J=2.8,1 \mathrm{H}, \mathrm{OH}) ; 1.71\left(\mathrm{~d}, \mathrm{~J}=6.4,3 \mathrm{H}, \mathrm{H}_{3} \mathrm{C}(8)\right) ; 1.33$ (d, $J=$ 6.9, $\left.3 \mathrm{H}, \mathrm{H}_{3} \mathrm{C}(1)\right) ; 1.14$ (d, $J=7.1,3 \mathrm{H}, \mathrm{H}_{3} \mathrm{C}\left(1^{\prime}\right)$ ); 0.92 (s, $9 \mathrm{H}, \mathrm{H}_{3} \mathrm{C}\left(3^{\prime \prime \prime}\right)$ ); 0.09 (s, $\left.6 \mathrm{H}, \mathrm{H}_{3} \mathrm{C}\left(1^{\prime \prime \prime}\right)\right)$

${ }^{13} \mathrm{C} \mathrm{NMR}:\left(\mathrm{CDCl}_{3}, 125 \mathrm{MHz}\right)$

$218.0(\mathrm{C}(3)) ; 130.6(\mathrm{C}(6)) ; 127.8(\mathrm{C}(7)) ; 74.6(\mathrm{C}(2)) ; 72.2(\mathrm{C}(5)) ; 45.1$ (C(4)); $25.7\left(\mathrm{C}\left(3^{\prime \prime \prime}\right)\right) ; 21.3(\mathrm{C}(1)) ; 18.1(\mathrm{C}(8)) ; 17.8\left(\mathrm{C}\left(2^{\prime \prime \prime}\right)\right) ; 11.1\left(\mathrm{C}\left(1^{\prime}\right)\right)$; -4.7 (C(1"')); - 
$5.0\left(\mathrm{C}\left(1^{\prime \prime \prime}\right)\right)$

MS: (FI)

$286\left(\mathrm{M}^{+}, 11\right), 229(100), 159(4), 110(2)$

IR: (neat)

3460 (br), 2956 (m), 2933 (m), 2858 (m), 1712 (m), 1461 (m), 1255 (m), 1120 (m), $966(\mathrm{~m}), 935(\mathrm{~m}), 835(\mathrm{~s}), 777(\mathrm{~m})$

TLC: $\quad R_{f} 0.13$ (pentane/ $\mathrm{Et}_{2} \mathrm{O}, 6 / 1$, anisaldehyde)

Opt. Rot.: $\quad[\alpha]_{\mathrm{D}}^{23}-3.7^{\circ}\left(c=2.00, \mathrm{CHCl}_{3}\right)$

SFC: $\quad t_{R}(2 S, 4 R, 5 S)-47,2.0$ min (Daicel Chiralpak AD, 5\% MeOH in $\mathrm{CO}_{2}, 150$ bar, 40 ${ }^{\circ} \mathrm{C}, 2.0 \mathrm{~mL} \min ^{-1}$ )

Analysis: $\quad \mathrm{C}_{15} \mathrm{H}_{30} \mathrm{O}_{3} \mathrm{Si}(286.49)$

Calculated: $\mathrm{C}, 62.89 ; \quad \mathrm{H}, 10.56 \%$

Found: $\quad$ C, $62.64 ; \quad H, 10.71 \%$

\section{(-)-(2S,4R,5R)-5-Hydroxy-4-methyl-7-phenyl-2-[((dimethyl)-(1,1-dimethylethyl)silyl)oxy]-6- heptyn-3-one (41h)}

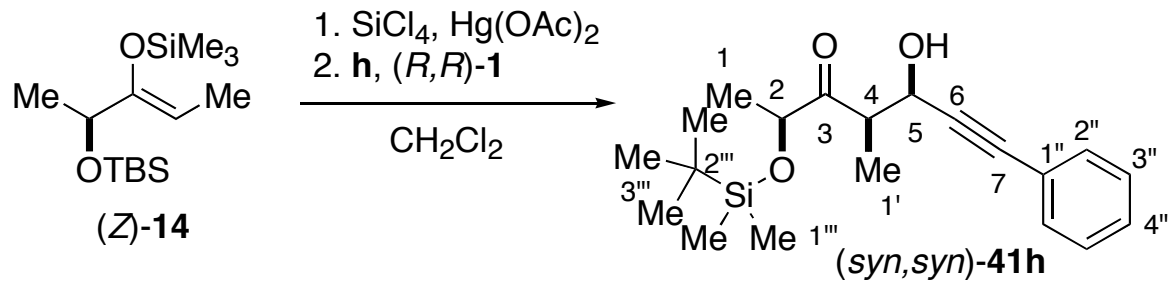

Following General Procedure VI: from silyl enol ether (Z)-14 (273 mg, $1.0 \mathrm{mmol})$, silicon tetrachloride $(230 \mu \mathrm{L}, 2.0 \mathrm{mmol}, 2.0$ equiv) and mercuric acetate $(3.2 \mathrm{mg}, 0.01 \mathrm{mmol}$, 0.01 equiv) in $\mathrm{CH}_{2} \mathrm{Cl}_{2}(1.0 \mathrm{~mL})$ followed by $(R, R)-\mathbf{1}\left(18 \mathrm{mg}, 0.05 \mathrm{mmol}, 0.05\right.$ equiv) in $\mathrm{CH}_{2} \mathrm{Cl}_{2}$ $(2.0 \mathrm{~mL})$ and 3-phenyl propynal $(122 \mu \mathrm{L}, 1.0 \mathrm{mmol})$ was obtained after chromatography (silica gel, pentane/ $\left.\mathrm{Et}_{2} \mathrm{O}, 4 / 1\right), 274 \mathrm{mg}(79 \%)$ of $\mathbf{4 1 h}$ as a clear, colorless oil. The diastereomeric ratio was determined to be (syn,syn)-41h/minor isomers, 95/3/2 by SFC analysis.

Data for 41h:

${ }^{1} \mathrm{H}$ NMR: $\quad\left(\mathrm{CDCl}_{3}, 500 \mathrm{MHz}\right)$

7.46-7.26 (m, $\left.5 \mathrm{H}, 2 \times \mathrm{H}\left(\mathrm{C}^{\prime \prime}\right), 2 \times \mathrm{HC}\left(3^{\prime \prime}\right), \mathrm{HC}\left(4^{\prime \prime}\right)\right)$; 4.92 (t, $\left.J=4.5,1 \mathrm{H}, \mathrm{HC}(5)\right)$; 
$4.31(\mathrm{q}, J=6.9,1 \mathrm{H}, \mathrm{HC}(2)) ; 3.41$ (dq, $J=6.9,4.1,1 \mathrm{H}, \mathrm{HC}(4)) ; 3.01$, (d, $J=4.7$, $1 \mathrm{H}, \mathrm{OH}) ; 1.41\left(\mathrm{~d}, J=6.6,3 \mathrm{H}, \mathrm{H}_{3} \mathrm{C}(1)\right) ; 1.41\left(\mathrm{~d}, J=6.9,3 \mathrm{H}, \mathrm{H}_{3} \mathrm{C}\left(1^{\prime}\right)\right) ; 0.96$ (s, 9 $\left.\mathrm{H}, \mathrm{H}_{3} \mathrm{C}\left(3^{\prime \prime \prime}\right)\right) ; 0.14$ (s, $\left.3 \mathrm{H}, \mathrm{H}_{3} \mathrm{C}\left(1^{\prime \prime \prime}\right)\right) ; 0.13$ (s, $\left.3 \mathrm{H}, \mathrm{H}_{3} \mathrm{C}\left(1^{\prime \prime \prime}\right)\right)$

${ }^{13} \mathrm{C} \mathrm{NMR}: \quad\left(\mathrm{CDCl}_{3}, 125 \mathrm{MHz}\right)$

216.4 (C(3)); 131.8 (CAr); 128.5 (CAr); 128. (CAr); 122.5 (C(1")); 87.8 (C(6));

85.3 (C(7)); 74.5 (C(2)); 63.5 (C(5)); 46.8 (C(4)); 25.7 (C(3"')); 21.2 (C(1)); 18.0 $(\mathrm{C}(8)) ; 18.0\left(\mathrm{C}\left(2^{\prime \prime \prime}\right)\right) ; 11.6\left(\mathrm{C}\left(1^{\prime}\right)\right) ;-4.6\left(\mathrm{C}\left(1^{\prime \prime \prime}\right)\right) ;-5.1\left(\mathrm{C}\left(1^{\prime \prime \prime}\right)\right)$

MS: $\quad(\mathrm{FI})$

$346\left(\mathrm{M}^{+}, 1\right), 289(100), 216(1), 187(2), 159(7), 145(5), 130(2)$

IR: (neat)

3440 (br), 2954 (s), 2931 (s), 2858 (m), 1714 (m), 1462 (m), 1365 (m), 1255 (m), $1126(\mathrm{~m}), 935(\mathrm{~m}), 835(\mathrm{~s}), 779(\mathrm{~s}), 758(\mathrm{~s}), 692(\mathrm{~m})$

TLC: $\quad R_{f} 0.24$ (pentane/ $\mathrm{Et}_{2} \mathrm{O}, 4 / 1$, anisaldehyde)

Opt. Rot:: $\quad[\alpha]_{\mathrm{D}}^{23}-1.2^{\circ}\left(c=2.00, \mathrm{CHCl}_{3}\right)$

SFC: $\quad t_{R}(2 S, 4 R, 5 S)-\mathbf{4 1 h}, 5.7 \mathrm{~min}$ (Daicel Chiralpak AD, 2\% $\mathrm{MeOH}$ in $\mathrm{CO}_{2}, 150$ bar, 40 $\left.{ }^{\circ} \mathrm{C}, 3.0 \mathrm{~mL} \min ^{-1}\right)$

Analysis: $\quad \mathrm{C}_{20} \mathrm{H}_{30} \mathrm{O}_{3} \mathrm{Si}(346.54)$

Calculated: $\quad$ C, 69.32; H, $8.73 \%$

Found: $\quad$ C, $69.17 ; \quad$ H, $8.96 \%$

\section{(-)-(2S,4R,5R)-5-Hydroxy-4-methyl-5-(2-naphthyl)-2-[((dimethyl)-(1,1-dimethylethyl)} silyl)oxy]-3-pentanone (411)

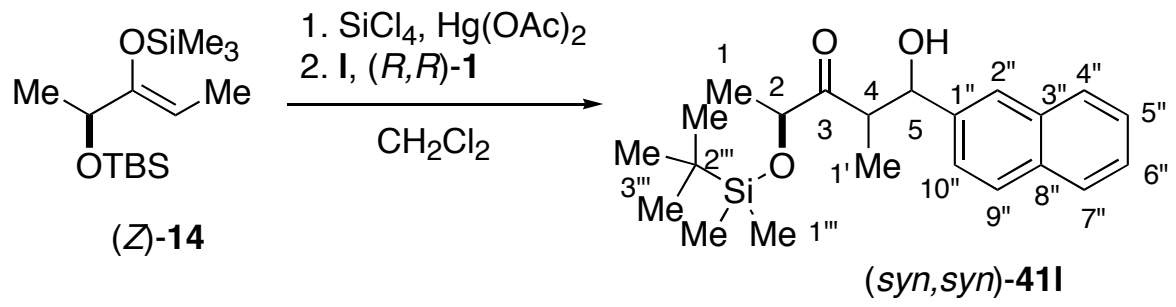

Following General Procedure VI: from silyl enol ether (Z)-14 (273 mg, $1.0 \mathrm{mmol})$, silicon tetrachloride $(230 \mu \mathrm{L}, 2.0 \mathrm{mmol}, 2.0$ equiv) and mercuric acetate $(3.2 \mathrm{mg}, 0.01 \mathrm{mmol}$, 0.01 equiv) in $\mathrm{CH}_{2} \mathrm{Cl}_{2}(1.0 \mathrm{~mL})$ followed by $(R, R)-1$ (18 mg, $0.05 \mathrm{mmol}, 0.05$ equiv) in $\mathrm{CH}_{2} \mathrm{Cl}_{2}$ 
(2.0 mL) and 2-naphthaldehyde (156 mg, $1.0 \mathrm{mmol}$ ) was obtained after chromatography (silica gel, pentane/ $\left.\mathrm{Et}_{2} \mathrm{O}, 4 / 1\right), 265 \mathrm{mg}(71 \%)$ of $\mathbf{4 1 l}$ as a clear, colorless oil. The diastereomeric ratio was determined to be (syn,syn)-411/ minor isomers, 94/3/3 by SFC analysis.

Data for 411:

${ }^{1} \mathrm{H} \mathrm{NMR}: \quad\left(\mathrm{CDCl}_{3}, 500 \mathrm{MHz}\right)$

7.86-7.36 (m, 7 H, HC(1"), HC(3"), HC(4"), HC(6"), HC(7"), HC(8"), HC(9")); 5.23 (br s, $1 \mathrm{H}, \mathrm{HC}(5)$ ); 4.21 (q, $J=6.9,1 \mathrm{H}, \mathrm{HC}(2)) ; 3.51$ (dq, $J=7.1,3.2,1 \mathrm{H}$, $\mathrm{HC}(4)) ; 3.46,(\mathrm{~d}, J=1.9,1 \mathrm{H}, \mathrm{OH}) ; 1.32$ (d, $\left.J=6.9,3 \mathrm{H}, \mathrm{H}_{3} \mathrm{C}(1)\right) ; 1.09$ (d, $J=$ 7.1, $\left.3 \mathrm{H}, \mathrm{H}_{3} \mathrm{C}\left(1^{\prime}\right)\right)$ ) 0.93 (s, $9 \mathrm{H}, \mathrm{H}_{3} \mathrm{C}\left(3^{\prime \prime \prime}\right)$ ); 0.11 (s, $3 \mathrm{H}, \mathrm{H}_{3} \mathrm{C}\left(1^{\prime \prime \prime}\right)$ ); 0.09 (s, $3 \mathrm{H}$, $\left.\mathrm{H}_{3} \mathrm{C}\left(1^{\prime \prime \prime}\right)\right)$

${ }^{13} \mathrm{C} \mathrm{NMR}: \quad\left(\mathrm{CDCl}_{3}, 125 \mathrm{MHz}\right)$

219.1 (C(3)); 139.0 (C(2")); 133.2 (C(10")); 132.7 (C(5")); 128.1 (CAr); 127.9 (CAr); 127.6 (CAr); 126.1 (CAr); 125.8 (CAr); 124.9 (CAr); 123.9 (CAr); 74.6 $(\mathrm{C}(2)) ; 72.77(\mathrm{C}(5)) ; 46.58$ (C(4)); 25.68 (C(3"')); 21.15 (C(1)); 18.01 (C(2"')); $10.21\left(\mathrm{C}\left(1^{\prime}\right)\right) ;-4.70\left(\mathrm{C}\left(1^{\prime \prime \prime}\right)\right) ;-5.01\left(\mathrm{C}\left(1^{\prime \prime \prime}\right)\right)$

MS: $\quad(\mathrm{FI})$

$372\left(\mathrm{M}^{+}, 100\right), 315(8), 267(15), 216(4), 156(10)$

IR: (neat) 3487 (br), 2954 (m), 2931 (m), 2858 (m), 1711 (m), 1462 (m), 1363 (m), 1255 (m), $1124(\mathrm{~m}), 935(\mathrm{~m}), 835$ (s), $779(\mathrm{~m}), 735$ (m)

TLC: $\quad R_{f} 0.26$ (pentane/ $\mathrm{Et}_{2} \mathrm{O}, 4 / 1$, anisaldehyde)

Opt. Rot.: $\quad[\alpha]_{\mathrm{D}}^{23}-1.7^{\circ}\left(c=2.00, \mathrm{CHCl}_{3}\right)$

SFC: $\quad t_{R}(2 S, 4 R, 5 R)-411,2.5$ min (Daicel Chiralpak AS, 4\% $\mathrm{MeOH}$ in $\mathrm{CO}_{2}, 150$ bar, 40 ${ }^{\circ} \mathrm{C}, 3.0 \mathrm{~mL} \min ^{-1}$ )

Analysis: $\quad \mathrm{C}_{22} \mathrm{H}_{32} \mathrm{O}_{3} \mathrm{Si}(372.58)$

Calculated: $\quad$ C, $70.92 ; \quad H, 8.66 \%$

Found: C, 70.62; H, $8.78 \%$ 
(-)-(2S,4R,5R)-5-Hydroxy-4-methyl-5-(1-furyl)-2-[((dimethyl)-(1,1-dimethylethyl)silyl)oxy]3-pentanone (41n)

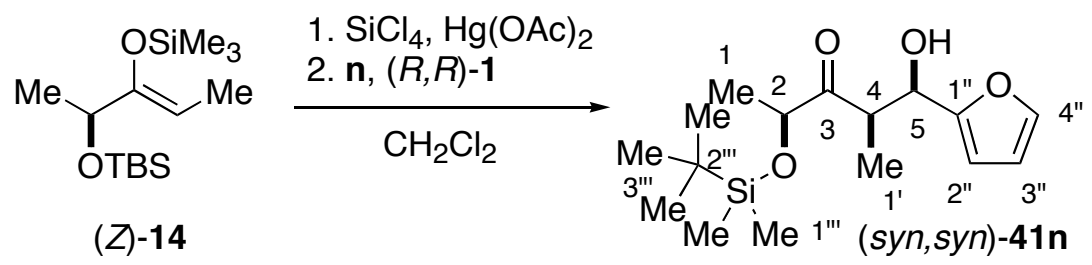

Following General Procedure II: from silyl enol ether (Z)-14 (273 mg, $1.0 \mathrm{mmol})$, silicon tetrachloride $(230 \mu \mathrm{L}, 2.0 \mathrm{mmol}, 2.0$ equiv) and mercuric acetate $(3.2 \mathrm{mg}, 0.01 \mathrm{mmol}, 0.01$ equiv) in $\mathrm{CH}_{2} \mathrm{Cl}_{2}(1.0 \mathrm{~mL})$ followed by $(R, R)-1$ (18 mg, $0.05 \mathrm{mmol}, 0.05$ equiv) in $\mathrm{CH}_{2} \mathrm{Cl}_{2}(2.0$ $\mathrm{mL}$ ) and 2-furaldehyde $(83 \mu \mathrm{L}, 1.0 \mathrm{mmol}$ ) was obtained after chromatography (silica gel, pentane/ $\left.\mathrm{Et}_{2} \mathrm{O}, 6 / 1\right), 257 \mathrm{mg}(82 \%)$ of $\mathbf{4 1} \mathrm{n}$ as a clear, colorless oil. The diastereomeric ratio was determined to be (syn,syn)-41n/minor isomers, 94/6 by SFC analysis.

Data for 41n:

${ }^{1}$ H NMR: $\quad\left(\mathrm{CDCl}_{3}, 500 \mathrm{MHz}\right)$

$7.34\left(\mathrm{~d}, J=1.7,1 \mathrm{H}, \mathrm{HC}\left(4^{\prime \prime}\right)\right) ; 6.33\left(\mathrm{dd}, J=3.2,1.7,1 \mathrm{H}, \mathrm{HC}\left(3^{\prime \prime}\right)\right) ; 6.27$ (d, J = $3.2,1 \mathrm{H}, \mathrm{HC}\left(2^{\prime \prime}\right) ; 5.03$ (t, J = 4.1, $\left.1 \mathrm{H}, \mathrm{HC}(5)\right) ; 4.20$ (q, J= 7.1, $\left.1 \mathrm{H}, \mathrm{HC}(2)\right) ; 3.58$ $(\mathrm{dq}, J=7.1,4.1,1 \mathrm{H}, \mathrm{HC}(4)) ; 2.95,(\mathrm{~d}, J=3.6,1 \mathrm{H}, \mathrm{OH}) ; 1.31(\mathrm{~d}, J=6.6,3 \mathrm{H}$, $\left.\mathrm{H}_{3} \mathrm{C}(1)\right) ; 1.18$ (d, $\left.J=7.2,3 \mathrm{H}, \mathrm{H}_{3} \mathrm{C}\left(1^{\prime}\right)\right) ; 0.95$ (s, $\left.9 \mathrm{H}, \mathrm{H}_{3} \mathrm{C}\left(3^{\prime \prime \prime}\right)\right) ; 0.12$ (s, $3 \mathrm{H}$, $\left.\mathrm{H}_{3} \mathrm{C}\left(1^{\prime \prime \prime}\right)\right) ; 0.11$ (s, $\left.3 \mathrm{H}, \mathrm{H}_{3} \mathrm{C}\left(1^{\prime \prime \prime}\right)\right)$

${ }^{13} \mathrm{C}$ NMR: $\quad\left(\mathrm{CDCl}_{3}, 125 \mathrm{MHz}\right)$

217.6 (C(3)); $154.4\left(\mathrm{C}\left(1^{\prime \prime}\right)\right) ; 141.7\left(\mathrm{C}\left(4^{\prime \prime}\right)\right) ; 110.3$ (C(3")); $106.6\left(\mathrm{C}\left(2^{\prime \prime}\right)\right) ; 74.4$ $(\mathrm{C}(2)) ; 68.3(\mathrm{C}(5)) ; 44.7$ (C(4)); 25.7 (C(3"')); 21.0 (C(1)); 18.0 (C(2"')); 11.3 $\left(\mathrm{C}\left(1^{\prime}\right)\right) ;-4.7\left(\mathrm{C}\left(1^{\prime \prime \prime}\right)\right) ;-5.1\left(\mathrm{C}\left(1^{\prime \prime \prime}\right)\right)$

MS: (FI)

$312\left(\mathrm{M}^{+}, 44\right), 255$ (100), 209 (8), 159 (16), 96 (6)

IR: (neat)

3467 (br), 2956 (m), 2933 (m), 2858 (m), 1714 (m), 1255 (m), 1126 (m), 1006 (m), $931(\mathrm{~m}), 835(\mathrm{~s}), 779(\mathrm{~m})$

TLC: $\quad R_{f} 0.13$ (pentane/ $\mathrm{Et}_{2} \mathrm{O}, 6 / 1$, anisaldehyde) 
Opt. Rot.: $\quad[\alpha]_{\mathrm{D}}^{23}-1.2^{\circ}\left(c=2.00, \mathrm{CHCl}_{3}\right)$

SFC: $\quad t_{R}(2 S, 4 R, 5 R)-41 \mathrm{n}, 4.0$ min (Daicel Chiralpak AD, $1.5 \% \mathrm{MeOH}$ in $\mathrm{CO}_{2}, 150$ bar, $40{ }^{\circ} \mathrm{C}, 2.5 \mathrm{~mL} \mathrm{~min}^{-1}$ )

Analysis: $\quad \mathrm{C}_{16} \mathrm{H}_{28} \mathrm{O}_{3} \mathrm{Si}(312.48)$

Calculated: $\mathrm{C}, 61.50 ; \quad \mathrm{H}, 9.03 \%$

Found: $\quad$ C, $61.30 ; \quad$ H, $9.04 \%$

General Procedure VII: Aldol Addition of (Z)-24 and 25: $(1 R, 2 R, 4 S)-5$-tertButyldimethylsilyloxy-2,4-dimethyl-1-hydroxy-1-phenyl-3-pentanone (syn,syn-45a)<smiles>CCOCC(C)C(=CC(C)C)OCC</smiles>

$(Z)-24$
$+\mathrm{PhCHO}$

a

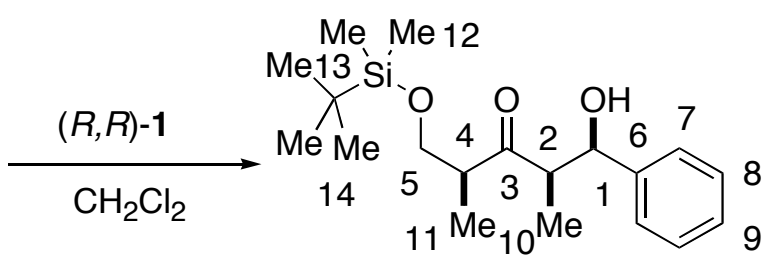

(syn,syn)-45a

In a $10-\mathrm{mL}$ Schlenk flask was placed a solution of $(R, R)-\mathbf{1}(37 \mathrm{mg}, 0.10 \mathrm{mmol}, 0.10$ equiv) in $\mathrm{CH}_{2} \mathrm{Cl}_{2}(1 \mathrm{~mL})$. To the solution was added $(Z)-24(364 \mathrm{mg}, 1.00 \mathrm{mmol})$ and the solution was cooled to $-70{ }^{\circ} \mathrm{C}$ using an acetone $/ \mathrm{CO}_{2}$ bath. To the solution was added dropwise $\mathrm{PhCHO}\left(102 \mu \mathrm{L}, 1.00 \mathrm{mmol}, 1\right.$ equiv) and the reaction mixture was stirred for $6 \mathrm{~h}$ at $-70{ }^{\circ} \mathrm{C}$. The reaction mixture was quenched by pouring into a vigorously stirring cold saturated $\mathrm{NaHCO}_{3}$ aq $(5 \mathrm{~mL})$. The resulting slurry was stirred for $1 \mathrm{~h}$ and filtered through Celite. The layers were separated, and the aqueous layer was extracted with $\mathrm{CH}_{2} \mathrm{Cl}_{2}(2 \times 10 \mathrm{~mL})$. The combined organic extrates were washed with brine $(5 \mathrm{~mL})$, dried over sodium sulfate and concentrated under reduced pressure. The crude product was purified by column chromatography (silica gel, pentane/ether, 3/1, $30 \mathrm{~mm})$ to afford $241 \mathrm{mg}(0.72 \mathrm{mmol}, 72 \%)$ of $\mathbf{4 5 a}$ as a clear viscous oil.

\section{Data for $(s y n, s y n)-45 \mathbf{a}$ :}

${ }^{1}$ H NMR: $\quad\left(500 \mathrm{MHz}, \mathrm{CDCl}_{3}\right)$

7.34-7.32 (m, $4 \mathrm{H}, \mathrm{HC}(7), \mathrm{HC}(8)) ; 7.25$ (m, $1 \mathrm{H}, \mathrm{HC}(9)) ; 5.22$ (t, J = 2.4, $1 \mathrm{H}$, $\mathrm{HC}(1))$; 3.75-3.60 (ABX, $\left.2 \mathrm{H}, \mathrm{H}_{2} \mathrm{C}(5)\right)$; 3.45 (d, $\left.J=2.4,1 \mathrm{H}, \mathrm{HO}\right) ; 3.02$ (sext, $J=$ 5.4, $1 \mathrm{H}, \mathrm{HC}(4)) ; 2.97$ (qd, $J=7.1,2.7,1 \mathrm{H}, \mathrm{HC}(2)) ; 1.02(\mathrm{~d}, J=6.8,3 \mathrm{H}$, $\left.\mathrm{H}_{3} \mathrm{C}(11)\right) ; 1.01$ (d, $\left.J=7.3,3 \mathrm{H}, \mathrm{H}_{3} \mathrm{C}(10)\right) ; 0.89$ (s, 9H, $\left.\mathrm{H}_{3} \mathrm{C}(14)\right)$; 0.06 (d, $J=3.2$, 
$\left.6 \mathrm{H}, \mathrm{H}_{3} \mathrm{C}(12)\right)$

${ }^{13} \mathrm{C} \mathrm{NMR:} \quad\left(126 \mathrm{MHz}, \mathrm{CDCl}_{3}\right)$

219.2 (C(3)); 141.7 (C(6)); 128.1 (C(8)); 127.1 (C(9)); 125.8 (C(7)); 71.9 (C(5)); $66.3(\mathrm{C}(1)) ; 52.8(\mathrm{C}(2)) ; 47.3(\mathrm{C}(4)) ; 25.9(\mathrm{C}(14)) ; 18.3(\mathrm{C}(13)) ; 13.2(\mathrm{C}(11)) ; 8.8$ $(\mathrm{C}(10)) ;-5.6(\mathrm{C}(12))$

IR: (neat)

3469 (br, w); 2954 (m); 2931 (m); 2858 (m); 1702 (m); 1462 (m); 1389 (w); 1255 (m); $1097(\mathrm{~m})$

MS: $\quad(\mathrm{FI})$

$336\left(\mathrm{M}^{+}, 40\right) ; 279$ (100); 265 (24); $230(12) ; 173(6) ; 159(3) ; 106(8)$

Opt. Rot.: $\quad[\alpha]_{\mathrm{D}}^{24}-9.16(\mathrm{EtOH}, \mathrm{c}=2.0)$

TLC: $\quad R_{f} 0.33$ (pentane/ether, 3/1) [silica gel, p-anisaldehyde]

SFC: $\quad t_{R}=1.834 \mathrm{~min}(\mathrm{AD}, 150 \mathrm{bar}, 3 \mathrm{~mL} / \mathrm{min}, 5 \% \mathrm{MeOH})$

Analysis: $\quad \mathrm{C}_{19} \mathrm{H}_{32} \mathrm{O}_{3} \mathrm{Si}(336.54)$

Calcd: $\quad$ C, $67.81 \% ; \quad \mathrm{H}, 9.58 \%$

Found: $\quad$ C, $67.74 \% ; \quad H, 9.69 \%$

\section{(1S, 2S, 4S)-5-tert-Butyldimethylsilyloxy-2,4-dimethyl-1-hydroxy-1-phenyl-3-pentanone} (syn,anti-45a)

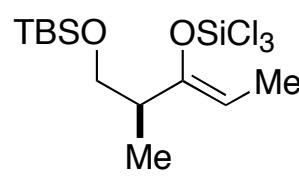

$(Z)-24$
$+\mathrm{PhCHO}$

a

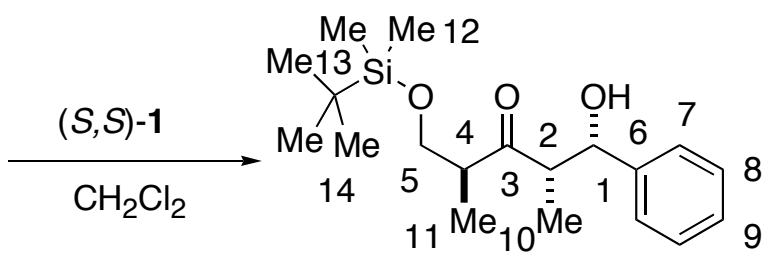

(syn,anti)-45a

Following General Procedure VII using (Z)-24 (364 mg, $1.00 \mathrm{mmol})$, PhCHO (102 $\mu \mathrm{L}$, $1.00 \mathrm{mmol})$ and $(S S)-1(37 \mathrm{mg}, 0.10 \mathrm{mmol})$ in $1 \mathrm{~mL}$ of $\mathrm{CH}_{2} \mathrm{Cl}_{2}, 241 \mathrm{mg}(0.72 \mathrm{mmol}, 72 \%)$ of 45a was obtained as a clear viscous oil after column chromatography (silica gel, pentane/ether, 3/1). 


\section{Data for (syn,anti)-45a:}

${ }^{1}$ H NMR: $\quad\left(500 \mathrm{MHz}, \mathrm{CDCl}_{3}\right)$

$7.34(\mathrm{~d}, J=4.2,4 \mathrm{H}, \mathrm{HC}(7), \mathrm{HC}(8)) ; 7.25$ (m, 1H, HC(9)); 5.05 (t, $J=3.2,1 \mathrm{H}$, $\mathrm{HC}(1)) ; 3.81-3.53\left(\mathrm{ABX}, 2 \mathrm{H}, \mathrm{H}_{2} \mathrm{C}(5)\right) ; 3.23$ (d, $\left.J=2.2,1 \mathrm{H}, \mathrm{HO}\right) ; 2.95$ (qd, $J=$ 6.9, 2.9, 1H, HC(2)); 2.89 (sext, $J=5.4,1 \mathrm{H}, \mathrm{HC}(4)) ; 1.04$ (d, $J=7.1,3 \mathrm{H}$, $\left.\mathrm{H}_{3} \mathrm{C}(11)\right) ; 0.89$ (d, $\left.J=6.6,3 \mathrm{H}, \mathrm{H}_{3} \mathrm{C}(10)\right) ; 0.87$ (s, 9H, $\left.\mathrm{H}_{3} \mathrm{C}(14)\right) ; 0.03$ (d, $J=6.4$, $\left.6 \mathrm{H}, \mathrm{H}_{3} \mathrm{C}(12)\right)$

${ }^{13} \mathrm{C} \mathrm{NMR}: \quad\left(126 \mathrm{MHz}, \mathrm{CDCl}_{3}\right)$

218.1 (C(3)); 142.1 (C(6)); 128.2 (C(8)); 127.3 (C(9)); 126.1 (C(7)); 73.5 (C(5)); $65.3(\mathrm{C}(1)) ; 52.8(\mathrm{C}(2)) ; 48.0(\mathrm{C}(4)) ; 25.8(\mathrm{C}(14)) ; 18.2(\mathrm{C}(13)) ; 12.9$ (C(11)); 9.9 $(\mathrm{C}(10)) ;-5.6(\mathrm{C}(12))$

IR: (neat)

3442 (br, m); 3064 (w); 3032 (w); 2956 (s); 2932 (s); 2859 (s); 1706 (s); 1462 (m); 1388 (m); 1361 (m); 1257 (s); 1099 (s); 1001 (s)

MS: $\quad(\mathrm{FI})$

$336\left(\mathrm{M}^{+}, 58\right) ; 279$ (100); $230(13) ; 173(5) ; 106(6)$

Opt. Rot:: $\quad[\alpha]_{\mathrm{D}}^{24}+49.34(\mathrm{EtOH}, \mathrm{c}=3.8)$

TLC: $\quad R_{f} 0.33$ (pentane/ether, 3/1) [silica gel, $p$-anisaldehyde]

SFC: $\quad t_{R}=2.278 \mathrm{~min}(\mathrm{AD}, 150 \mathrm{bar}, 3 \mathrm{~mL} / \mathrm{min}, 5 \% \mathrm{MeOH})$

Analysis: $\quad \mathrm{C}_{19} \mathrm{H}_{32} \mathrm{O}_{3} \mathrm{Si}(336.54)$

Calcd: $\quad$ C, $67.81 \% ; \quad \mathrm{H}, 9.58 \%$

Found: $\quad$ C, $67.74 \% ; \quad H, 9.73 \%$ 
$(1 R, 2 R, 4 S)-5$-Triisopropylsilyloxy-2,4-dimethyl-1-hydroxy-1-phenyl-3-pentanone (syn,syn46a)

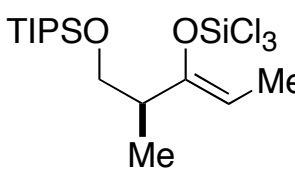

(Z)-25
$+\mathrm{PhCHO}$

a

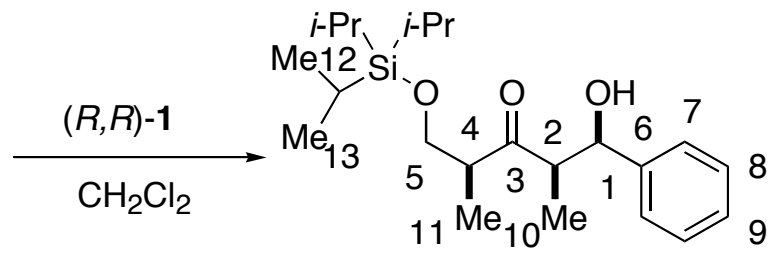

(syn,syn)-46a

Following General Procedure VII using (Z)-25 (479 mg, $1.00 \mathrm{mmol})$, PhCHO (102 $\mu \mathrm{L}$, $1.00 \mathrm{mmol})$ and $(R, R)-\mathbf{1}(37 \mathrm{mg}, 0.10 \mathrm{mmol})$ in $1 \mathrm{~mL}$ of $\mathrm{CH}_{2} \mathrm{Cl}_{2}$ for $8 \mathrm{~h}, 319 \mathrm{mg}(0.84 \mathrm{mmol}$, $84 \%$ ) of 46a was obtained as a clear viscous oil after column chromatography (silica gel, pentane/ether, 5/1).

Data for $(s y n, s y n)-\mathbf{4 6 a}$ :

${ }^{1}$ H NMR: $\quad\left(500 \mathrm{MHz}, \mathrm{CDCl}_{3}\right)$

7.35-7.31 (m, $4 \mathrm{H}, \mathrm{HC}(7), \mathrm{HC}(8)) ; 7.25$ (m, $1 \mathrm{H}, \mathrm{HC}(9)) ; 5.23$ (t, $J=2.5,1 \mathrm{H}$, $\mathrm{HC}(1)$ ); 3.85-3.67 (ABX, $\left.2 \mathrm{H}, \mathrm{H}_{2} \mathrm{C}(5)\right)$ ) 3.47 (d, $\left.J=2.2,1 \mathrm{H}, \mathrm{HO}\right) ; 3.01$ (sext, $J=$ 5.4, $1 \mathrm{H}, \mathrm{HC}(4)) ; 2.98$ (qd, $J=7.3,2.7,1 \mathrm{H}, \mathrm{HC}(2)) ; 1.14-1.02$ (m, $27 \mathrm{H}$, $\left.\mathrm{H}_{3} \mathrm{C}(11), \mathrm{H}_{3} \mathrm{C}(10), \mathrm{H}_{3} \mathrm{C}(13), \mathrm{HC}(12)\right)$

${ }^{13} \mathrm{C} \mathrm{NMR}: \quad\left(126 \mathrm{MHz}, \mathrm{CDCl}_{3}\right)$

$219.8(\mathrm{C}(3)) ; 141.9(\mathrm{C}(6)) ; 128.4(\mathrm{C}(8)) ; 127.3(\mathrm{C}(9)) ; 126.1(\mathrm{C}(7)) ; 72.1(\mathrm{C}(5))$; $67.0(\mathrm{C}(1)) ; 53.1(\mathrm{C}(4)) ; 47.8(\mathrm{C}(2)) ; 18.2(\mathrm{C}(13)) ; 13.5(\mathrm{C}(11)) ; 12.0(\mathrm{C}(12)) ; 9.1$ (C(10))

IR: (neat) 3467 (br, m); 2942 (s); 2891 (s); 2868 (s); 1703 (s); 1462 (s); 1385 (m); 1101 (s); 1070 (m); 997 (s)

MS: $\quad(\mathrm{FI})$

$379\left(\mathrm{M}^{+}, 6\right) ; 337(7) ; 336(26) ; 335$ (100)

Opt. Rot: $\quad[\alpha]_{\mathrm{D}}^{24}-10.84^{\circ}(\mathrm{EtOH}, \mathrm{c}=1.01)$

TLC: $\quad R_{f} 0.23$ (pentane/ether, 5/1) [silica gel, $p$-anisaldehyde]

SFC: $\quad t_{R}=2.220 \mathrm{~min}(\mathrm{AD}, 150 \mathrm{bar}, 3 \mathrm{~mL} / \mathrm{min}, 5 \% \mathrm{MeOH})$ 
Analysis: $\quad \mathrm{C}_{22} \mathrm{H}_{38} \mathrm{O}_{3} \mathrm{Si}(378.62)$

Calcd: $\quad$ C, $69.79 \% ; \quad H, 10.12 \%$

Found: $\quad$ C, $69.53 \% ; \quad H, 10.24 \%$

(1S, 2S, 4S)-1-Triisopropylsilyloxy-2,4-dimethyl-5-hydroxy-5-phenyl-3-pentanone (syn,anti46a)

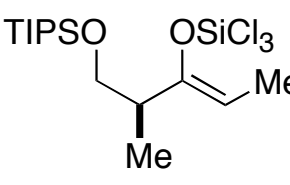

$(Z)-25$
$+\mathrm{PhCHO}$

a

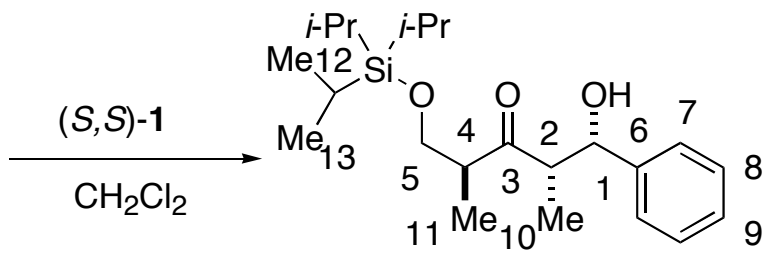

(syn, anti)-46a

Following General Procedure VII using (Z)-25 (479 mg, $1.00 \mathrm{mmol})$, PhCHO (102 $\mu \mathrm{L}$, $1.00 \mathrm{mmol})$ and $(S, S)-\mathbf{1}(37 \mathrm{mg}, 0.10 \mathrm{mmol})$ in $1 \mathrm{~mL}$ of $\mathrm{CH}_{2} \mathrm{Cl}_{2}$ for $8 \mathrm{~h}, 310 \mathrm{mg}(0.82 \mathrm{mmol}$, $82 \%$ ) of 46a was obtained as a clear viscous oil after column chromatography (silica gel, pentane/ether, 5/1).

Data for (syn,anti)-46a:

mp: $\quad 22{ }^{\circ} \mathrm{C}$ (Hexane)

${ }^{1} \mathrm{H} \mathrm{NMR}: \quad\left(500 \mathrm{MHz}, \mathrm{CDCl}_{3}\right)$

7.34-7.25 (m, 5 H, HC(7), $\mathrm{HC}(8), \mathrm{HC}(9)) ; 5.06$ (dd, $J=3.9,2.4,1 \mathrm{H}, \mathrm{HC}(1))$; 3.91-3.61 (ABX, $\left.2 \mathrm{H}, \mathrm{H}_{2} \mathrm{C}(5)\right) ; 3.18$ (d, $\left.J=2.2,1 \mathrm{H}, \mathrm{HO}\right) ; 2.99$ (qd, $J=7.1,4.1,1$ H, HC(2)); 2.89 (sext, $J=5.1,1 \mathrm{H}, \mathrm{HC}(4)) ; 1.08$ (d, $J=7.1,3 \mathrm{H}, \mathrm{H}_{3} \mathrm{C}(11)$ ); 1.09 - $1.02\left(\mathrm{~m}, 21 \mathrm{H}, \mathrm{H}_{3} \mathrm{C}(13), \mathrm{HC}(12)\right) ; 0.91$ (d, $J=7.1,3 \mathrm{H}, \mathrm{H}_{3} \mathrm{C}(10)$ )

${ }^{13} \mathrm{C} \mathrm{NMR}: \quad\left(126 \mathrm{MHz}, \mathrm{CDCl}_{3}\right)$

$218.3(\mathrm{C}(3)) ; 141.9(\mathrm{C}(6)) ; 128.2(\mathrm{C}(8)) ; 127.3(\mathrm{C}(9)) ; 126.0(\mathrm{C}(7)) ; 73.4(\mathrm{C}(5))$; 65.6 (C(1)); 52.9 (C(4)); 48.3 (C(2)); 17.9 (C(13)); 13.2 (C(11)); 11.9 (C(12)); 9.6 $(\mathrm{C}(10))$

IR: (neat)

3367 (br, m); 2943 (s); 2866 (s); 1707 (s); 1462 (s); 1285 (m); 1265 (m); 1248 (w); 1132 (m); 1097 (s); 1065 (s); 1003 (s) 
$\underline{\mathrm{MS}}: \quad(\mathrm{FI})$

$379\left(\mathrm{M}^{+}, 5\right) ; 337$ (7); 336 (25); 335 (100); 272 (10); 229 (14); 157 (5); 157 (7);

157 (7); 127 (7); 157 (6); $156(7) ; 106$ (6)

Opt. Rot.: $\quad[\alpha]_{\mathrm{D}}^{24}+92.03(\mathrm{EtOH}, \mathrm{c}=0.59)$

TLC: $\quad R_{f} 0.18$ (pentane/ether, 5/1) [silica gel, $p$-anisaldehyde]

SFC: $\quad t_{R}=2.900 \mathrm{~min}(\mathrm{AD}, 150 \mathrm{bar}, 3 \mathrm{~mL} / \mathrm{min}, 5 \% \mathrm{MeOH})$

Analysis: $\quad \mathrm{C}_{22} \mathrm{H}_{38} \mathrm{O}_{3} \mathrm{Si}(378.62)$

Calcd: $\quad$ C, $69.79 \%$;, $10.12 \%$

Found: $\quad$ C, $69.67 \%$; H, $9.95 \%$

(1R, 2R, 4S)-5-Triisopropylsilyloxy-2,4-dimethyl-1-hydroxy-1-(1-naphthyl)-3-pentanone (syn,syn-46b)

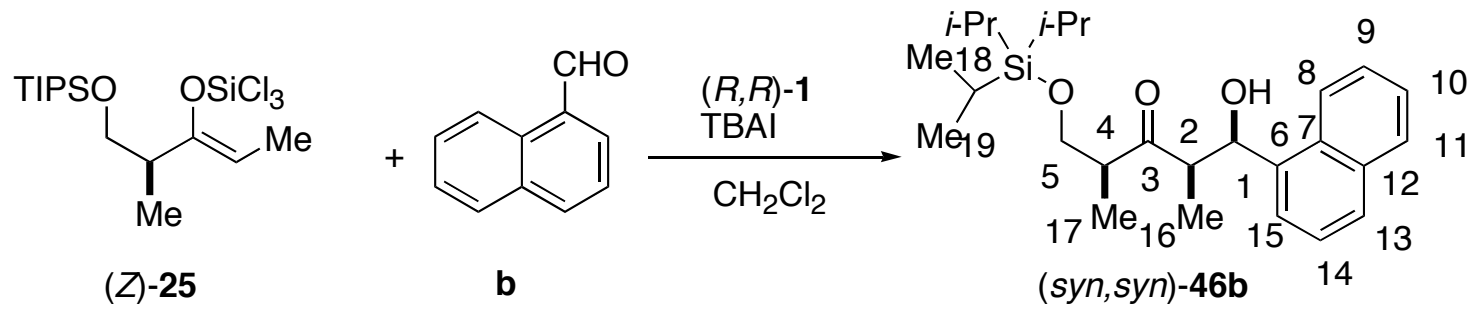

Following General Procedure VII using (Z)-25 (472 mg, $1.00 \mathrm{mmol}), 1$-naphthaldehyde $(136 \mu \mathrm{L}, 1.00 \mathrm{mmol}),(R, R)-1(37 \mathrm{mg}, 0.10 \mathrm{mmol})$ and TBAI $(73.9 \mathrm{mg}, 0.20 \mathrm{mmol})$ in $1 \mathrm{~mL}$ of $\mathrm{CH}_{2} \mathrm{Cl}_{2}$ for $8 \mathrm{~h}, 313 \mathrm{mg}(0.71 \mathrm{mmol}, 71 \%)$ of $\mathbf{4 6 \mathbf { b }}$ was obtained as a clear viscous oil after column chromatography (silica gel, pentane/ether, 5/1). Analytically pure material was obtained by recrystalization from pentane.

Data for $(s y n, s y n)-\mathbf{4 6 b}$ :

mp: $\quad 50-52^{\circ} \mathrm{C}$ (pentane)

${ }^{1}$ H NMR: $\quad\left(500 \mathrm{MHz}, \mathrm{CDCl}_{3}\right)$

$7.89-7.88$ (m, $2 \mathrm{H}, \mathrm{HC}(8), \mathrm{HC}(11)) ; 7.77$ (d, $J=10,1 \mathrm{H}, \mathrm{HC}(13)) ; 7.76$ (d, $J=$ 10, $1 \mathrm{H}, \mathrm{HC}(15)) ; 7.51$ (t, $J=7.4,1 \mathrm{H}, \mathrm{HC}(9)) ; 7.55-7.42$ (m, $2 \mathrm{H}, \mathrm{HC}(14)$, $\mathrm{HC}(10)$ ); 6.03 (br s, $1 \mathrm{H}, \mathrm{HC}(1)) ; 3.95-3.72$ (ABX, 2 H, H $\left.\mathrm{H}_{2} \mathrm{C}(5)\right)$; 3.66 (d, $J=2.4$, $1 \mathrm{H}, \mathrm{HO}) ; 3.21(\mathrm{qd}, J=7.1,2.5,1 \mathrm{H}, \mathrm{HC}(2)) ; 3.10(\mathrm{sextd}, J=6.8,1.7,1 \mathrm{H}$, 
$\mathrm{HC}(4)) ; 1.13$ - 1.00 (m, $\left.27 \mathrm{H}, \mathrm{H}_{3} \mathrm{C}(13), \mathrm{H}_{3} \mathrm{C}(16), \mathrm{HC}(18), \mathrm{H}_{3} \mathrm{C}(19)\right)$

${ }^{13} \mathrm{C} \mathrm{NMR:} \quad\left(126 \mathrm{MHz}, \mathrm{CDCl}_{3}\right)$

219.5 (C(3)); 136.8 (C(6)); 134.0 (C(12)); 129.9 (C(7)); 129.5 (C(11)); 128.0 (C(13)); 126.2 (C(10)); 125.7 (C(9)); 125.6 (C(14)); 124.8 (C(15)); 122.6 (C(8)); 68.7 (C(1)); 66.7 (C(5); 51.1 (C(4)); 48.2 (C(2)); 18.3 (C(19)); 14.1 (C(17)); 12.2 (C(18)); $9.6(\mathrm{C}(16))$

IR: $\quad\left(\mathrm{CH}_{2} \mathrm{Cl}_{2}\right)$

3469 (br, m); 3052 (w); 2943 (s); 2867 (s); 1704 (m); 1598 (w); 1511 (w); 1462 (m); $1383(\mathrm{w}) ; 1253(\mathrm{w}) ; 1101(\mathrm{~m}) ; 1068(\mathrm{~m})$

$\underline{\mathrm{MS}}: \quad(\mathrm{FI})$

429 (14); $428\left(\mathrm{M}^{+}, 37\right) ; 385$ (12); 336 (25); 272 (14); 230 (17); 229 (100); 156 (69)

Opt. Rot.: $\quad[\alpha]_{\mathrm{D}}^{24}+2.1(\mathrm{EtOH}, \mathrm{c}=0.48)$

TLC: $\quad R_{f} 0.30$ (pentane/ether, 4/1) [silica gel, short UV]

SFC: $\quad t_{R}=5.555 \mathrm{~min}(\mathrm{OD}, 150 \mathrm{bar}, 3 \mathrm{~mL} / \mathrm{min}, 15 \% \mathrm{MeOH})$

Analysis: $\quad \mathrm{C}_{26} \mathrm{H}_{40} \mathrm{O}_{3} \mathrm{Si}(428.68)$

Calcd: $\quad$ C, $72.85 \% ; \quad H, 9.41 \%$

Found: $\quad$ C, $72.52 \% ; \quad H, 9.40 \%$

\section{(1S, 2S, 4S)-5-Triisopropylsilyloxy-2,4-dimethyl-1-hydroxy-1-(1-naphthyl)-3-pentanone} (syn,anti-46b)

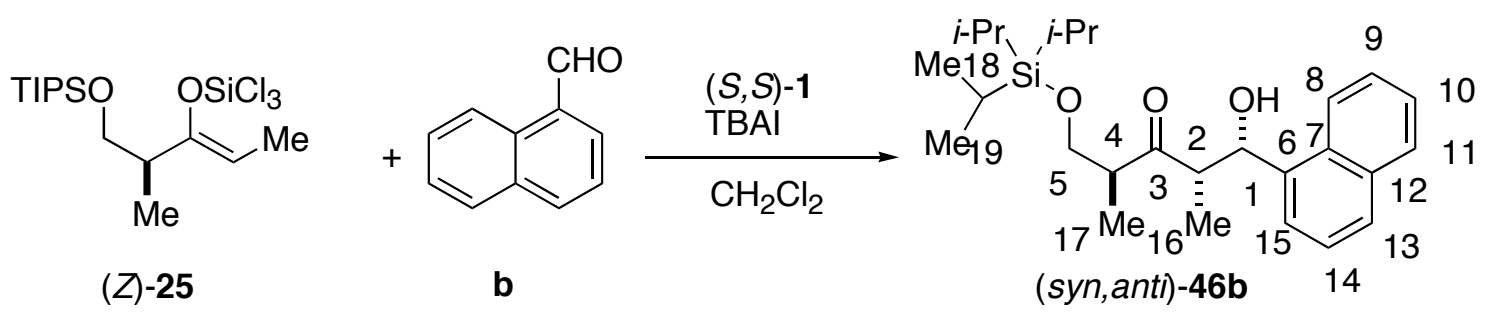

Following General Procedure VII using (Z)-25 (501 mg, 1.00 mmol, 19\% bisenoxysilane), 1-naphthaldehyde (136 $\mu \mathrm{L}, 1.00 \mathrm{mmol}),(S, S)-1(37 \mathrm{mg}, 0.10 \mathrm{mmol})$ and TBAI (73.9 mg, $0.20 \mathrm{mmol})$ in $1 \mathrm{~mL}$ of $\mathrm{CH}_{2} \mathrm{Cl}_{2}$ for $8 \mathrm{~h}, 335 \mathrm{mg}(0.78 \mathrm{mmol}, 78 \%)$ of $46 \mathbf{b}$ was obtained 
as a clear viscous oil after column chromatography (silica gel, pentane/ether, 5/1).

\section{Data for (syn,anti)-46b:}

${ }^{1}$ H NMR: $\quad\left(500 \mathrm{MHz}, \mathrm{CDCl}_{3}\right)$

$7.92(\mathrm{~d}, J=8.0,1 \mathrm{H}, \mathrm{HC}(8)) ; 7.89$ (d, $J=7.4,1 \mathrm{H}, \mathrm{HC}(11)) ; 7.78(\mathrm{~d}, J=8.0,1$ $\mathrm{H}, \mathrm{HC}(13)) ; 7.74$ (d, $J=7.3,1 \mathrm{H}, \mathrm{HC}(15)) ; 7.53$ - 7.47 (m, 3 H, HC(10), HC(14), $\mathrm{C}(9)$ ); 5.90 (br d, $J=2.2,1 \mathrm{H}, \mathrm{HC}(1)) ; 3.99-3.66$ (ABX, $2 \mathrm{H}, \mathrm{H}_{2} \mathrm{C}(5)$ ); 3.57 (d, $J$ $=2.2,1 \mathrm{H}, \mathrm{HO}) ; 3.20(\mathrm{qd}, J=7.0,1.7,1 \mathrm{H}, \mathrm{HC}(2)) ; 3.00(\mathrm{sextd}, J=7.1,1.7,1 \mathrm{H}$, $\mathrm{HC}(4)) ; 1.12-0.98\left(\mathrm{~m}, 27 \mathrm{H}, \mathrm{H}_{3} \mathrm{C}(17), \mathrm{H}_{3} \mathrm{C}(19), \mathrm{HC}(18), \mathrm{H}_{3} \mathrm{C}(16)\right)$

${ }^{13} \mathrm{C} \mathrm{NMR}: \quad\left(126 \mathrm{MHz}, \mathrm{CDCl}_{3}\right)$

219.0 (C(3)); $136.7(\mathrm{C}(6)) ; 133.7$ (C(12)); 129.7 (C(7)); 129.1 (C(11)); 127.8 (C(13)); 126.1 (C(10)); 125.4 (C(9)); 125.3 (C(14)); 124.4 (C(15)); 122.4 (C(8));

69.7 (C(1)); 65.7 (C(5)); 50.1 (C(4)); 48.1 (C(2)); 17.9 (C(19)); 15.3 (C(17)); 11.9 (C(18)); $9.3(\mathrm{C}(16))$

IR: (neat)

3470 (br, m); 3052 (w); 2943 (s); 2892 (m); 1703 (m); 1598 (w); $1511(\mathrm{w}) ; 1462$ (m); $1383(\mathrm{~m}) ; 1253(\mathrm{w}) ; 1102(\mathrm{~s}) ; 1068(\mathrm{~m})$

MS: (FI)

430 (13); 429 (26); $428\left(\mathrm{M}^{+}, 48\right) ; 385$ (58); 347 (10); 273 (13); 272 (46); 230 (13); 229 (92); 156 (100)

Opt. Rot.: $\quad[\alpha]_{\mathrm{D}}^{24}+47.9(\mathrm{EtOH}, \mathrm{c}=0.97)$

TLC: $\quad R_{f} 0.26$ (pentane/ether, 5/1) [silica gel, short UV]

SFC: $\quad t_{R}=3.44 \mathrm{~min}(\mathrm{OD}, 150 \mathrm{bar}, 3 \mathrm{~mL} / \mathrm{min}, 15 \% \mathrm{MeOH})$

Analysis: $\quad \mathrm{C}_{26} \mathrm{H}_{40} \mathrm{O}_{3} \mathrm{Si}(428.68)$
Calcd:
C, $72.85 \%$;
$\mathrm{H}, 9.41 \%$
Found:
C, $72.47 \%$;
H, $9.29 \%$ 
(2S, 4R, 5R)-1-Triisopropylsilyloxy-2,4-dimethyl-5-hydroxy-7-phenyl-6-hepten-3-one (syn,syn-46c)

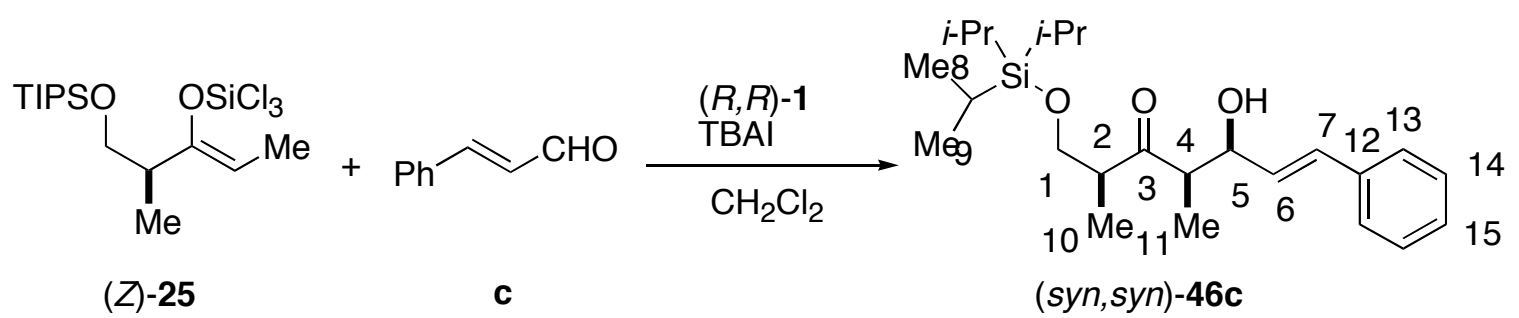

Following General Procedure VII using (Z)-25 (872 mg, $2.00 \mathrm{mmol})$, cinnamaldehyde (252 $\mu \mathrm{L}, 2.00 \mathrm{mmol}),(R, R)-1(74 \mathrm{mg}, 0.20 \mathrm{mmol})$ and TBAI $(148 \mathrm{mg}, 0.40 \mathrm{mmol})$ in $2 \mathrm{~mL}$ of $\mathrm{CH}_{2} \mathrm{Cl}_{2}$ for $10 \mathrm{~h}, 713 \mathrm{mg}(1.76 \mathrm{mmol}, 88 \%)$ of $\mathbf{4 6 c}$ was obtained as a clear viscous oil after column chromatography (silica gel, pentane/ether, 3/1).

Data for (syn,syn)-46c:

${ }^{1} \mathrm{H}$ NMR: $\quad\left(500 \mathrm{MHz}, \mathrm{CDCl}_{3}\right)$

$7.38(\mathrm{~d}, J=7.1,2 \mathrm{H}, \mathrm{HC}(13)) ; 7.31(\mathrm{t}, J=7.5,2 \mathrm{H}, \mathrm{HC}(14)) ; 7.24(\mathrm{t}, J=7.4,1$ H, HC(15)); 6.64 (dd, $J=15.8,0.9,1 \mathrm{H}, \mathrm{HC}(7)) ; 6.13$ (dd, $J=15.8,5.6,1 \mathrm{H}$, $\mathrm{HC}(6))$; 4.75 (m, $1 \mathrm{H}, \mathrm{HC}(5))$; 3.88-3.70 (ABX, $\left.2 \mathrm{H}, \mathrm{H}_{2} \mathrm{C}(1)\right)$; 3.30 (d, J = 2.9, 1 $\mathrm{H}, \mathrm{HO}) ; 3.09$ (sext, $J=5.4,1 \mathrm{H}, \mathrm{HC}(2)) ; 2.95$ (qd, $J=7.3,2.7,1 \mathrm{H}, \mathrm{HC}(4)$ ); 1.17 $\left(\mathrm{d}, J=7.4,3 \mathrm{H}, \mathrm{H}_{3} \mathrm{C}(11)\right) ; 1.11-1.02$ (m, $\left.24 \mathrm{H}, \mathrm{H}_{3} \mathrm{C}(9), \mathrm{H}_{3} \mathrm{C}(10), \mathrm{HC}(8)\right)$

${ }^{13} \mathrm{C} \mathrm{NMR}: \quad\left(126 \mathrm{MHz}, \mathrm{CDCl}_{3}\right)$

219.4 (C(3)); 137.1 (C(12)); 130.9 (C(6)); 129.2 (C(7)); 128.8 (C(14)); 127.8 $(\mathrm{C}(15)) ; 126.6(\mathrm{C}(13)) ; 71.5(\mathrm{C}(1)) ; 67.2(\mathrm{C}(5)) ; 51.5(\mathrm{C}(4)) ; 47.6(\mathrm{C}(2)) ; 18.2$ $(\mathrm{C}(9)) ; 13.4(\mathrm{C}(10)) ; 12.0(\mathrm{C}(8)) ; 9.6(\mathrm{C}(11))$

IR: (neat)

3436 (br, m); 3061 (w); 3027 (w); 2943 (s); 2892 (m); 2867 (m); 1703 (m); 1600 (w); 1495(w); 1462 (m); $1384(\mathrm{w}) ; 1368$ (w); 1249 (w); $1102(\mathrm{~m}) ; 1069$ (m)

MS: $\quad(\mathrm{FI})$

406 (9); 405 (34); 404 (M+1 100); 335 (9); 272 (8); 229 (24); 132 (48)

Opt. Rot.: $\quad[\alpha]_{\mathrm{D}}^{24}+20.0\left(\mathrm{CH}_{2} \mathrm{Cl}_{2}, \mathrm{c}=0.73\right)$

$[\alpha]_{\mathrm{D}}^{24}-47.8(\mathrm{EtOH}, \mathrm{c}=0.73)$ 
TLC: $\quad R_{f} 0.12$ (pentane/ether, 3/1) [silica gel, short UV]

SFC: $\quad t_{R}=3.348 \mathrm{~min}(\mathrm{OD}, 150 \mathrm{bar}, 3 \mathrm{~mL} / \mathrm{min}, 7 \% \mathrm{MeOH})$

Analysis: $\quad \mathrm{C}_{24} \mathrm{H}_{40} \mathrm{O}_{3} \mathrm{Si}(404.66)$

Calcd: $\quad$ C, $71.23 \%$; $\quad$ H, $9.96 \%$

Found: $\quad$ C, $71.27 \%$; $\quad \mathrm{H}, 10.08 \%$

\section{(2S, 4S, 5S)-1-Triisopropylsilyloxy-2,4-dimethyl-5-hydroxy-7-phenyl-6-hepten-3-one} (syn,anti-46c)

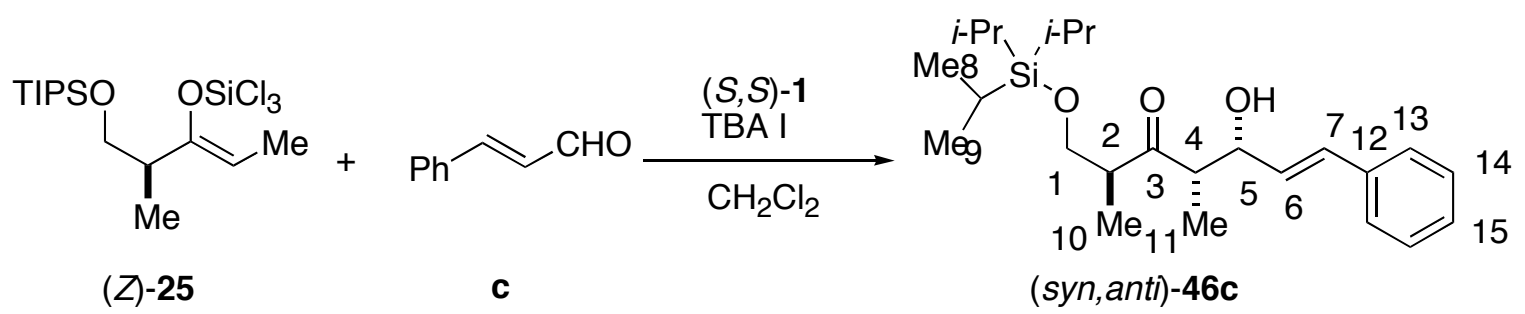

Following General Procedure VII using (Z)-25 (436 mg, $1.00 \mathrm{mmol})$, cinnamaldehyde $(126 \mu \mathrm{L}, 1.00 \mathrm{mmol}),(S, S)-1(37 \mathrm{mg}, 0.10 \mathrm{mmol})$ and TBAI $(73.9 \mathrm{mg}, 0.20 \mathrm{mmol})$ in $1 \mathrm{~mL}$ of $\mathrm{CH}_{2} \mathrm{Cl}_{2}$ for $10 \mathrm{~h}, 303 \mathrm{mg}(0.75 \mathrm{mmol}, 75 \%)$ of $\mathbf{4 6 c}$ was obtained as a clear viscous oil after column chromatography (silica gel, pentane/ether, 3/1).

Data for (syn,anti)-46c:

${ }^{1}$ H NMR: $\quad\left(500 \mathrm{MHz}, \mathrm{CDCl}_{3}\right)$

$7.38(\mathrm{~d}, J=7.6,2 \mathrm{H}, \mathrm{HC}(13)) ; 7.31$ (t, $J=7.6,2 \mathrm{H}, \mathrm{HC}(14)) ; 7.24$ (t, $J=7.8,1$ H, HC(15)); 6.65 (d, $J=16.1,1 \mathrm{H}, \mathrm{HC}(7)) ; 6.13$ (dd, $J=15.8,5.8,1 \mathrm{H}, \mathrm{HC}(6))$; 4.64 (br m, $1 \mathrm{H}, \mathrm{HC}(5)$ ); 3.94-3.65 (ABX, $2 \mathrm{H}, \mathrm{H}_{2} \mathrm{C}(1)$ ); 3.03 (sext, $J=5.4,1 \mathrm{H}$, $\mathrm{HC}(2)) ; 2.99$ (d, $J=2.9,1 \mathrm{H}, \mathrm{HO}$ ); 2.92 (qd, $J=7.3,3.6,1 \mathrm{H}, \mathrm{HC}(4)) ; 1.18$ (d, $J$ $\left.=6.5,3 \mathrm{H}, \mathrm{H}_{3} \mathrm{C}(11)\right) ; 1.16-1.04\left(\mathrm{~m}, 24 \mathrm{H}, \mathrm{H}_{3} \mathrm{C}(9), \mathrm{H}_{3} \mathrm{C}(10), \mathrm{HC}(8)\right)$

${ }^{13} \mathrm{C}$ NMR: $\quad\left(101 \mathrm{MHz}, \mathrm{CDCl}_{3}\right)$

$218.1(\mathrm{C}(3)) ; 137.1(\mathrm{C}(12)) ; 130.9(\mathrm{C}(6)) ; 129.1(\mathrm{C}(7)) ; 128.5(\mathrm{C}(14)) ; 127.6$ (C(15)); 126.4 (C(13)); 72.3 (C(1)); $65.9(\mathrm{C}(5)) ; 50.5$ (C(4)); 48.1 (C(2)); 17.9 $(\mathrm{C}(9)) ; 15.3(\mathrm{C}(10)) ; 11.8(\mathrm{C}(8)) ; 9.9(\mathrm{C}(11))$

IR: (neat) 3430 (br, m); 3083 (w); 3061 (w); 3027 (w); 2943 (s); 2892 (s); 2726 (w); 1706 
(s); $1627(\mathrm{w}) ; 1600(\mathrm{w}) ; 1578(\mathrm{w}) ; 1495(\mathrm{~m}) ; 1462$ (s); $1383(\mathrm{~m}) ; 1368(\mathrm{~m}) ; 1250$ (m); $1203(\mathrm{w}) ; 1104(\mathrm{~s}) ; 1068$ (s)

MS: $\quad(\mathrm{FI})$

405 (12); $404\left(\mathrm{M}^{+}, 34\right) ; 287$ (11); 273 (15); 269 (15); 261 (69); 245 (30); 229 (100); 217 (83); 132 (17); 106 (28)

Opt. Rot.: $\quad[\alpha]_{\mathrm{D}}^{24}+42.1\left(\mathrm{CH}_{2} \mathrm{Cl}_{2}, \mathrm{c}=1.35\right)$

$$
[\alpha]_{\mathrm{D}}^{24}+109.0(\mathrm{EtOH}, \mathrm{c}=1.25)
$$

TLC: $\quad R_{f} 0.23$ (pentane/ether, 3/1) [silica gel, short UV]

SFC: $\quad t_{R}=6.630 \mathrm{~min}(\mathrm{OD}, 150 \mathrm{bar}, 3 \mathrm{~mL} / \mathrm{min}, 7 \% \mathrm{MeOH})$

Analysis: $\quad \mathrm{C}_{24} \mathrm{H}_{40} \mathrm{O}_{3} \mathrm{Si}(404.66)$

$\begin{array}{lll}\text { Calcd: } & \text { C }, 71.23 \% ; & \text { H, } 9.96 \% \\ \text { Found: } & \text { C }, 71.30 \% ; & \text { H, } 9.79 \%\end{array}$

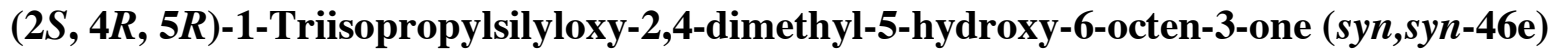

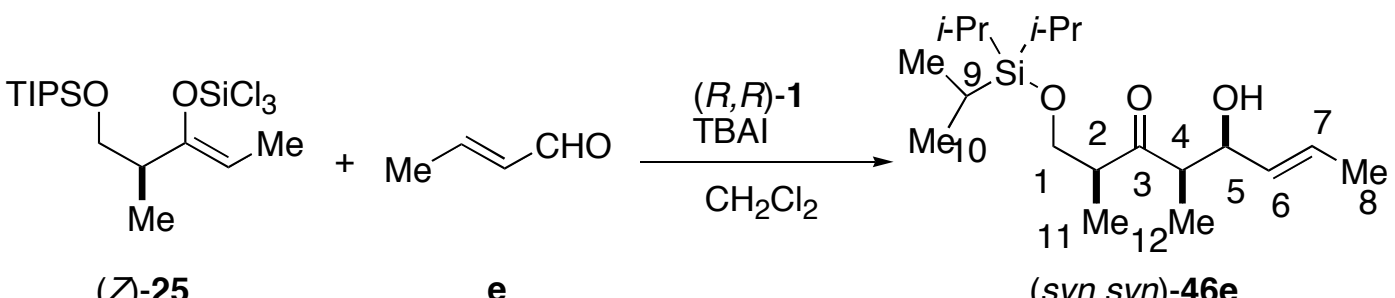

(Z)-25

(syn,syn)-46e

Following General Procedure VII using (Z)-25 (472 mg, $1.00 \mathrm{mmol})$, crotonaldehyde (83 $\mu \mathrm{L}, 1.00 \mathrm{mmol}),(R, R)-1(37 \mathrm{mg}, 0.10 \mathrm{mmol})$ and TBAI $(73.9 \mathrm{mg}, 0.20 \mathrm{mmol})$ in $1 \mathrm{~mL}$ of $\mathrm{CH}_{2} \mathrm{Cl}_{2}$ for $6 \mathrm{~h}, 310 \mathrm{mg}(0.90 \mathrm{mmol}, 90 \%)$ of $\mathbf{4 6 \mathbf { d }}$ was obtained as a clear viscous oil after column chromatography (silica gel, pentane/ether, 3/1).

Data for (syn,syn)-46e:

${ }^{1}$ H NMR: $\quad\left(500 \mathrm{MHz}, \mathrm{CDCl}_{3}\right)$

$5.70(\mathrm{dq}, J=16.4,6.0,1 \mathrm{H}, \mathrm{HC}(7)) ; 5.45(\mathrm{dd}, J=6.4,1.5,1 \mathrm{H}, \mathrm{HC}(6)) ; 4.47$ (m, $1 \mathrm{H}, \mathrm{HC}(5)$ ); 3.86-3.67 (ABX, $2 \mathrm{H}, \mathrm{H}_{2} \mathrm{C}(1)$ ); 3.04 (sext, $J=5.4,1 \mathrm{H}, \mathrm{HC}(2)$ ); 3.03 $(\mathrm{d}, J=3.2,1 \mathrm{H}, \mathrm{HO}) ; 2.83(\mathrm{qd}, J=7.3,2.9,1 \mathrm{H}, \mathrm{HC}(4)) ; 1.70(\mathrm{dd}, J=5.7,1.0,3$ $\left.\mathrm{H}, \mathrm{H}_{3} \mathrm{C}(8)\right) ; 1.12$ (d, $\left.J=7.4,3 \mathrm{H}, \mathrm{H}_{3} \mathrm{C}(11)\right) ; 1.10-1.07$ (m, $24 \mathrm{H}, \mathrm{H}_{3} \mathrm{C}(10)$, 


\section{$\left.\mathrm{H}_{3} \mathrm{C}(12), \mathrm{HC}(9)\right)$}

${ }^{13} \mathrm{C} \mathrm{NMR}: \quad\left(126 \mathrm{MHz}, \mathrm{CDCl}_{3}\right)$

$219.0(\mathrm{C}(3)) ; 130.4(\mathrm{C}(7)) ; 127.3(\mathrm{C}(6) ; 71.6(\mathrm{C}(1)) ; 66.6(\mathrm{C}(5)) ; 51.2$ (C(4));

47.5 (C(2)); 17.8 (C(10)); 17.7 (C(8)); 13.1 (C(11)); 11.7 (C(9)); $9.6(\mathrm{C}(12))$

IR: (neat)

3514 (br, w); 2945 (m); 2868 (m); 1699 (m); 1462 (m); 1383 (w); 1247 (w); 1098 $(\mathrm{m}) ; 1068(\mathrm{~m}) ; 1008(\mathrm{~m})$

MS: $\quad(\mathrm{FI})$

$343\left(\mathrm{M}^{+}, 5\right) ; 301(6) ; 300(25) ; 299$ (100); $272(6) ; 176(6) ; 176(9) ; 175(10) ; 175$ (8); 175 (6)

Opt. Rot.: $\quad[\alpha]_{\mathrm{D}}^{24}+5.06(\mathrm{EtOH}, \mathrm{c}=0.23)$

TLC: $\quad R_{f} 0.25$ (pentane/ether, 3/1) [silica gel, $p$-anisaldehyde]

Analysis: $\quad \mathrm{C}_{19} \mathrm{H}_{38} \mathrm{O}_{3} \mathrm{Si}(342.59)$
Calcd:
C, 66.61\%;
H, $11.18 \%$
Found:
C, 66.34\%;
H, $11.16 \%$

(2S, 4S, 5S)-1-Triisopropylsilyloxy-2,4-dimethyl-5-hydroxy-6-octen-3-one (syn,anti-46e)

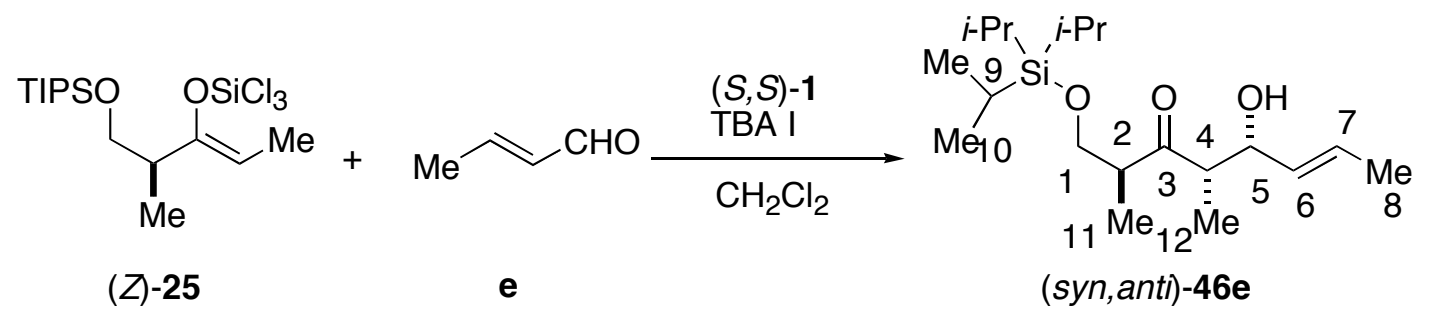

Following General Procedure VII using (Z)-25 (472 mg, $1.00 \mathrm{mmol})$, crotonaldehyde (83 $\mu \mathrm{L}, 1.00 \mathrm{mmol}),(S, S)-1(37 \mathrm{mg}, 0.10 \mathrm{mmol})$ and TBAI $(73.9 \mathrm{mg}, 0.20 \mathrm{mmol})$ in $1 \mathrm{~mL}$ of $\mathrm{CH}_{2} \mathrm{Cl}_{2}$ for $6 \mathrm{~h}, 293 \mathrm{mg}(0.85 \mathrm{mmol}, 85 \%)$ of 46e was obtained as a clear viscous oil after column chromatography (silica gel, pentane/ether, 3/1).

Data for (syn,anti)-46e:

${ }^{1} \mathrm{H}$ NMR: $\quad\left(500 \mathrm{MHz}, \mathrm{CDCl}_{3}\right)$

$5.72(\mathrm{dq}, J=15.2,6.4,1 \mathrm{H}, \mathrm{HC}(7)) ; 5.46(\mathrm{dd}, J=15.1,6.6,1 \mathrm{H}, \mathrm{HC}(6)) ; 4.36(\mathrm{~m}$, 
$1 \mathrm{H}, \mathrm{HC}(5)$ ); 3.91-3.63 (ABX, $2 \mathrm{H}, \mathrm{H}_{2} \mathrm{C}(1)$ ); 2.99 (sext, $J=7.6,1 \mathrm{H}, \mathrm{HC}(2)$ ); 2.81 (qd, $J=7.0,4.0,1 \mathrm{H}, \mathrm{HC}(4)) ; 2.66$ (d, $J=3.2,1 \mathrm{H}, \mathrm{HO}) ; 1.70$ (dd, $J=7.3,0.7,3$ $\left.\mathrm{H}, \mathrm{H}_{3} \mathrm{C}(8)\right) ; 1.12$ (d, J =7.1, $3 \mathrm{H}, \mathrm{H}_{3} \mathrm{C}(11)$ ); $1.11-1.00$ (m, $24 \mathrm{H}, \mathrm{H}_{3} \mathrm{C}(10)$, $\left.\mathrm{H}_{3} \mathrm{C}(12), \mathrm{HC}(9)\right)$

${ }^{13} \mathrm{C} \mathrm{NMR}: \quad\left(126 \mathrm{MHz}, \mathrm{CDCl}_{3}\right)$

$217.8(\mathrm{C}(3)) ; 130.9(\mathrm{C}(7)) ; 127.6(\mathrm{C}(6) ; 72.7$ (C(1)); $65.8(\mathrm{C}(5)) ; 50.8$ (C(4)); $48.2(\mathrm{C}(2)) ; 17.8(\mathrm{C}(10)) ; 17.6(\mathrm{C}(8)) ; 13.1(\mathrm{C}(11)) ; 11.8(\mathrm{C}(9)) ; 9.6(\mathrm{C}(12))$

IR: (neat)

3468 (br, w); 2944 (m); 2868 (m); 1703 (m); 1462 (m); 1383 (w); 1250 (w); 1104 (m); $1068(\mathrm{~m})$

MS: $\quad(\mathrm{FI})$

$342\left(\mathrm{M}^{+}, 1\right) ; 301(6) ; 300$ (23); 299 (100); 229 (15); 175 (7)

Opt. Rot.: $\quad[\alpha]_{\mathrm{D}}^{24}+81.7(\mathrm{EtOH}, \mathrm{c}=0.35)$

TLC: $\quad R_{f} 0.25$ (pentane/ether, 3/1) [silica gel, $p$-anisaldehyde]

Analysis: $\quad \mathrm{C}_{19} \mathrm{H}_{38} \mathrm{O}_{3} \mathrm{Si}(342.59)$

Calcd: $\quad$ C, $66.61 \% ; \quad H, 11.18 \%$

Found: $\quad$ C, $66.21 \% ; \quad H, 11.13 \%$

(2S, 4R, 5R)-1-Triisopropylsilyloxy-2,4,6-trimethyl-5-hydroxy-6-octen-3-one (syn,syn-46g)

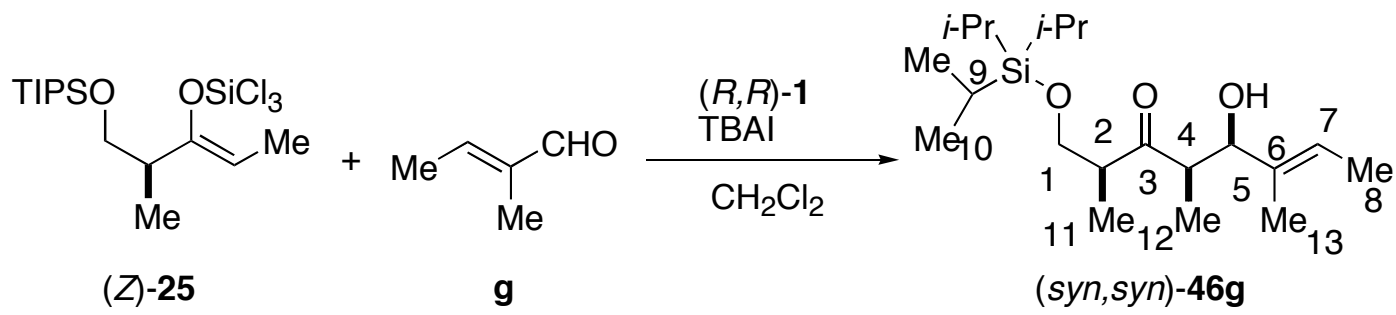

Following General Procedure VII using (Z)-25 (472 mg, $1.00 \mathrm{mmol}$ ), tiglicaldehyde (96 $\mu \mathrm{L}, 1.00 \mathrm{mmol}),(R, R)-1(37 \mathrm{mg}, 0.10 \mathrm{mmol})$ and TBAI $(73.9 \mathrm{mg}, 0.20 \mathrm{mmol})$ in $1 \mathrm{~mL}$ of $\mathrm{CH}_{2} \mathrm{Cl}_{2}$ for $7 \mathrm{~h}, 303 \mathrm{mg}(0.85 \mathrm{mmol}, 85 \%)$ of $\mathbf{4 6} \mathbf{g}$ was obtained as a clear viscous oil after column chromatography (silica gel, pentane/ether, 5/1). 
Data for $(s y n, s y n)-\mathbf{4 6 g}$ :

${ }^{1}$ H NMR: $\quad\left(500 \mathrm{MHz}, \mathrm{CDCl}_{3}\right)$

5.60 (qt, $J=6.8,1.5,1 \mathrm{H}, \mathrm{HC}(7)) ; 4.42$ (m, $1 \mathrm{H}, \mathrm{HC}(5))$; 3.87-3.67 (ABX, $2 \mathrm{H}$, $\left.\mathrm{H}_{2} \mathrm{C}(1)\right) ; 3.11(\mathrm{~d}, J=2.5,1 \mathrm{H}, \mathrm{HO}) ; 3.02$ (sext, $\left.J=6.8,1 \mathrm{H}, \mathrm{HC}(2)\right) ; 2.88$ (qd, $J=$ 7.3, 2.9, $1 \mathrm{H}, \mathrm{HC}(4)) ; 1.63$ (d, $\left.J=6.3,3 \mathrm{H}, \mathrm{H}_{3} \mathrm{C}(8)\right) ; 1.56$ (s, $\left.3 \mathrm{H}, \mathrm{H}_{3} \mathrm{C}(13)\right) ; 1.10$ - 1.02 (m, $\left.27 \mathrm{H}, \mathrm{H}_{3} \mathrm{C}(11), \mathrm{H}_{3} \mathrm{C}(10), \mathrm{H}_{3} \mathrm{C}(12), \mathrm{HC}(9)\right)$

${ }^{13} \mathrm{C}$ NMR: $\quad\left(126 \mathrm{MHz}, \mathrm{CDCl}_{3}\right)$

219.2 (C(3)); $133.7(\mathrm{C}(7)) ; 119.9(\mathrm{C}(6)) ; 73.8(\mathrm{C}(1)) ; 66.5(\mathrm{C}(5)) ; 48.6(\mathrm{C}(4))$; 47.5 (C(2)); 17.9 (C(10)); $13.4(\mathrm{C}(8)) ; 13.3$ (C(13)); 12.9 (C(11)); $11.8(\mathrm{C}(9))$; 9.0 (C(12))

IR: $\quad\left(\mathrm{CHCl}_{3}\right)$

3584 (br, w); 2945 (m); 2868 (m); 1701 (w); 1461 (w); 1383 (w); 1103 (w)

MS: $\quad(\mathrm{FI})$

$357\left(\mathrm{M}^{+}, 7\right) ; 311(5) ; 310(4) ; 231(5) ; 230$ (18); 229 (100)

Opt. Rot.: $\quad[\alpha]_{\mathrm{D}}^{24}+5.71(\mathrm{EtOH}, \mathrm{c}=0.20)$

TLC: $\quad R_{f} 0.20$ (pentane/ether, 5/1) [silica gel, $p$-anisaldehyde]

Analysis: $\quad \mathrm{C}_{20} \mathrm{H}_{40} \mathrm{O}_{3} \mathrm{Si}(356.62)$

Calcd: $\quad$ C, $67.36 \%$; $11.31 \%$

Found: $\quad$ C, $66.99 \% ; \quad H, 11.28 \%$

(2S, 4S, 5S)-1-Triisopropylsilyloxy-2,4,6-trimethyl-5-hydroxy-6-octen-3-one (syn,anti-46g)

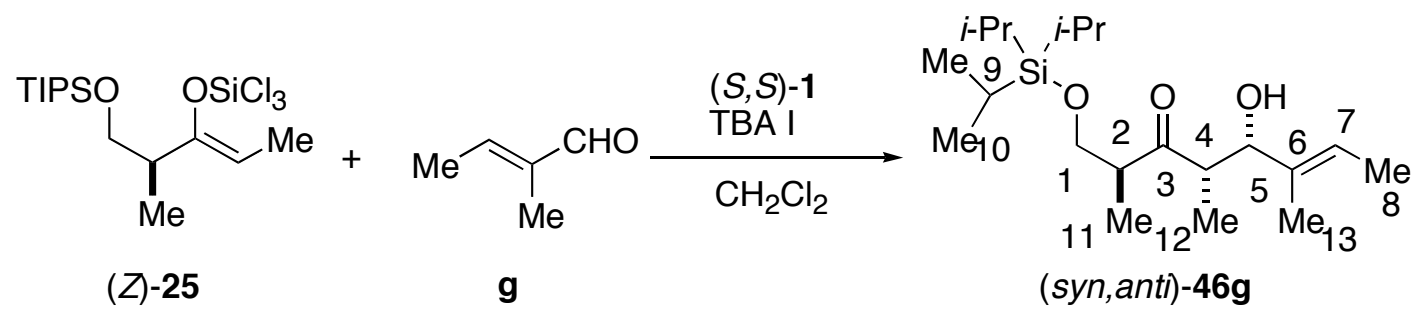

Following General Procedure VII using (Z)-25 (472 mg, $1.00 \mathrm{mmol}$ ), tiglicaldehyde (96 $\mu \mathrm{L}, 1.00 \mathrm{mmol}),(S, S)-\mathbf{1}(37 \mathrm{mg}, 0.10 \mathrm{mmol})$ and TBAI $(73.9 \mathrm{mg}, 0.20 \mathrm{mmol})$ in $1 \mathrm{~mL}$ of $\mathrm{CH}_{2} \mathrm{Cl}_{2}$ for $7 \mathrm{~h}, 286 \mathrm{mg}(0.80 \mathrm{mmol}, 80 \%)$ of $\mathbf{4 6} \mathbf{g}$ was obtained as a clear viscous oil after column 
chromatography (silica gel, pentane/ether, 5/1).

\section{Data for (syn,anti)-46g:}

${ }^{1}$ H NMR: $\quad\left(500 \mathrm{MHz}, \mathrm{CDCl}_{3}\right)$

5.57 (qt, $J=6.8,1.2,1 \mathrm{H}, \mathrm{HC}(7))$ ) 4.36 (br s, $1 \mathrm{H}, \mathrm{HC}(5))$; 3.89-3.63 (ABX, $2 \mathrm{H}$, $\mathrm{H}_{2} \mathrm{C}(1)$ ); 2.99 (sext, $\left.J=5.4,1 \mathrm{H}, \mathrm{HC}(2)\right) ; 2.92$ (qd, $\left.J=7.1,3.0,1 \mathrm{H}, \mathrm{HC}(4)\right) ; 2.64$ (d, $J=2.9,1 \mathrm{H}, \mathrm{HO}) ; 1.66$ (d, $J=6.9,3 \mathrm{H}, \mathrm{H}_{3} \mathrm{C}(8)$ ); 1.60 (s, $3 \mathrm{H}, \mathrm{H}_{3} \mathrm{C}(13)$ ); 1.10 $\left(\mathrm{m}, 27 \mathrm{H}, \mathrm{H}_{3} \mathrm{C}(11), \mathrm{H}_{3} \mathrm{C}(10), \mathrm{H}_{3} \mathrm{C}(12), \mathrm{HC}(9)\right)$

${ }^{13} \mathrm{C} \mathrm{NMR}: \quad\left(126 \mathrm{MHz}, \mathrm{CDCl}_{3}\right)$

217.9 (C(3)); $134.4(\mathrm{C}(6)) ; 120.4(\mathrm{C}(7)) ; 73.8$ (C(5)); 66.0 (C(1)); 48.6 (C(4)); $48.1(\mathrm{C}(2)) ; 17.9(\mathrm{C}(10)) ; 13.4(\mathrm{C}(8)) ; 13.2(\mathrm{C}(13)) ; 12.9(\mathrm{C}(11)) ; 11.8(\mathrm{C}(9)) ; 9.5$ (C(12))

IR: (neat)

3468 (br, m); 2944 (m); 2868 (m); 1703 (m); 1463 (m); 1383 (w); 1249 (w); 1102 (m); $1068(\mathrm{w})$

MS: $\quad(\mathrm{FI})$

$356\left(\mathrm{M}^{+}, 1\right) ; 311(5) ; 232(5) ; 231(6) ; 230$ (18); 229 (100)

Opt. Rot.: $\quad[\alpha]_{\mathrm{D}}^{24}+10.2(\mathrm{EtOH}, \mathrm{c}=0.53)$

TLC: $\quad R_{f} 0.20$ (pentane/ether, 5/1) [silica gel, $p$-anisaldehyde]

Analysis: $\quad \mathrm{C}_{20} \mathrm{H}_{40} \mathrm{O}_{3} \mathrm{Si}(356.62)$

Calcd: C $67.36 \%$; H $11.31 \%$

Found: C $66.96 \%$; H $11.30 \%$ 
General Procedure VIII: Aldol Additions of Trichlorosilyl Enolates 26 and 27: (1R, 2R, 5S)-5-tert-Butyldimethylsilyloxy-2-methyl-1-hydroxy-1-phenyl-3-hexanone (syn,anti-47a)

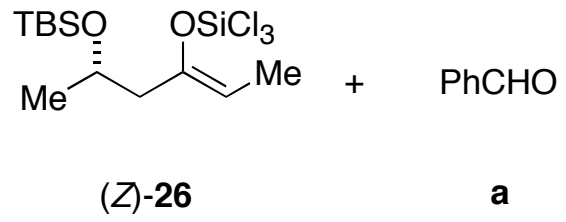

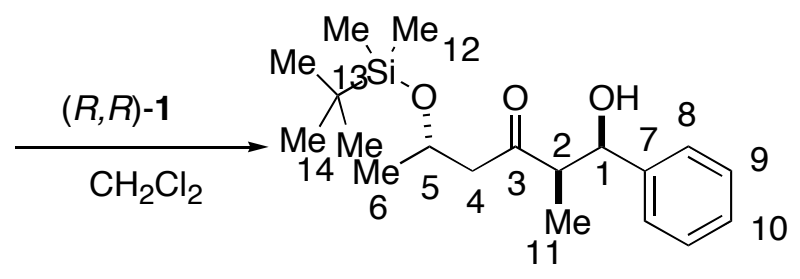

(syn,anti)-47a

In a $10-\mathrm{mL}$ Schlenk flask was placed a solution of $(R, R)-1(37 \mathrm{mg}, 0.10 \mathrm{mmol}, 0.10$ equiv) in $\mathrm{CH}_{2} \mathrm{Cl}_{2}(1 \mathrm{~mL})$. To the solution was added (Z)-26 (364 mg, $\left.1.00 \mathrm{mmol}\right)$ and the solution was cooled to $-68{ }^{\circ} \mathrm{C}$ using $i-\mathrm{PrOH} / \mathrm{CO}_{2}$ bath. To the solution was added $\mathrm{PhCHO}$ (102 $\mu \mathrm{L}, 1.00 \mathrm{mmol}, 1$ equiv) and the reaction mixture was stirred for $6 \mathrm{~h}$ at $-68{ }^{\circ} \mathrm{C}$. The reaction mixture was quenched by pouring into a vigorously stirring cold sat. aq. $\mathrm{NaHCO}_{3}$ solution $(15 \mathrm{~mL})$. The resulting slurry was stirred for $3 \mathrm{~h}$ and filtered through Celite. The layers were separated, and the aqueous layer was extracted with $\mathrm{CH}_{2} \mathrm{Cl}_{2}(2 \times 15 \mathrm{~mL})$. The combined extracts were washed with brine $(5 \mathrm{~mL})$, dried over sodium sulfate and concentrated. The crude oil was purified by column chromatography (silica gel, pentane/ether, 3/1, $30 \mathrm{~mm}$ ) to afford 200 $\mathrm{mg}(0.59 \mathrm{mmol}, 59 \%)$ of $\mathbf{4 7 \mathbf { a }}$ as a clear viscous oil.

Data for (syn,anti)-47a:

${ }^{1} \mathrm{H}$ NMR: $\quad\left(500 \mathrm{MHz}, \mathrm{CDCl}_{3}\right)$

7.36-7.30 (m, $4 \mathrm{H}, \mathrm{HC}(8), \mathrm{HC}(9)) ; 7.25$ (m, $1 \mathrm{H}, \mathrm{HC}(10)) ; 5.15$ (t, J = 2.9, $1 \mathrm{H}$, $\mathrm{HC}(1)) ; 4.32$ (sext, $J=6.6,1 \mathrm{H}, \mathrm{HC}(5)) ; 3.16$ (d, $J=2.6,1 \mathrm{H}, \mathrm{HO}) ; 2.81$ (qd, $J=$ 7.3, 3.1, $1 \mathrm{H}, \mathrm{HC}(2)) ; 2.72-2.43\left(\mathrm{ABX}, 2 \mathrm{H}, \mathrm{H}_{2} \mathrm{C}(4)\right) ; 1.16(\mathrm{~d}, J=6.1,3 \mathrm{H}$, $\left.\mathrm{H}_{3} \mathrm{C}(6)\right) ; 1.04$ (d, $\left.J=7.3,3 \mathrm{H}, \mathrm{H}_{3} \mathrm{C}(11)\right) ; 0.87$ (s, 9H, $\left.\mathrm{H}_{3} \mathrm{C}(14)\right) ; 0.06$ (d, $J=11.2$, $\left.6 \mathrm{H}, \mathrm{H}_{3} \mathrm{C}(12)\right)$

${ }^{13}$ C NMR: $\quad\left(126 \mathrm{MHz}, \mathrm{CDCl}_{3}\right)$ 214.7 (C(3)); $141.6(\mathrm{C}(7)) ; 128.2$ (C(9)); 127.2 (C(10)); 125.8 (C(8)); 72.3 (C(5)); 65.4 (C(1)); 52.5 (C(2)); 51.3 (C(4)); 25.8 (C(14)); 23.9 (C(6)); 17.9 (C(13)); 9.2 (C(11)); -4.6, -4.9 (C(12)) 
IR: $\quad$ (neat)

3458 (br, m); 3064 (w); 3032 (w); 2956 (m); 2931 (m); 2895 (m); 2858 (m); 1707 (m); 1454 (m); 1375 (m); 1255 (m); 1134 (m); 1095 (m); 1005 (m)

MS: $\quad(\mathrm{FI})$

$336\left(\mathrm{M}^{+}, 7\right) ; 279$ (100); $230(5) ; 173$ (3);107 (5)

Opt. Rot.: $\quad[\alpha]_{\mathrm{D}}^{24}-5.55(\mathrm{EtOH}, \mathrm{c}=0.63)$

TLC: $\quad R f 0.20$ (pentane/ether, 5/1) [silica gel, $p$-anisaldehyde]

SFC: $\quad t_{R}=2.441 \mathrm{~min}(\mathrm{OD}, 150 \mathrm{bar}, 3 \mathrm{~mL} / \mathrm{min}, 5 \% \mathrm{MeOH})$

Analysis: $\quad \mathrm{C}_{19} \mathrm{H}_{32} \mathrm{O}_{3} \mathrm{Si}(336.54)$

Calcd: $\quad$ C, $67.81 \% ; \quad \mathrm{H}, 9.58 \%$

Found: $\quad$ C, $67.61 \% ; \quad H, 9.59 \%$

\section{$(1 S, 2 S, 5 S)-5$-tert-Butyldimethylsilyloxy-2-methyl-1-hydroxy-1-phenyl-3-hexanone} (syn,syn-47a)

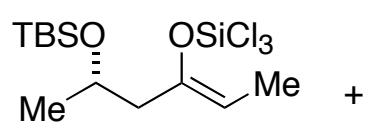

$(Z)-26$
$\mathrm{PhCHO}$

a

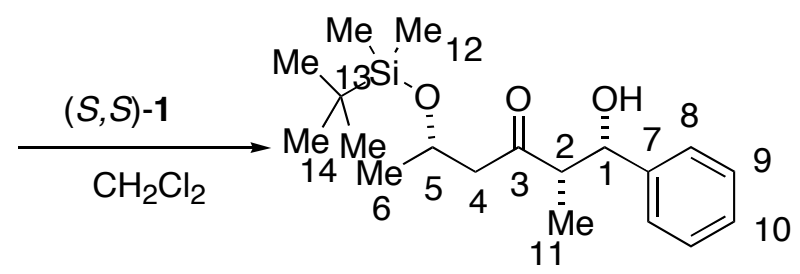

(syn,syn)-47a

Following General Procedure VIII using (Z)-26 (364 mg, $1.00 \mathrm{mmol})$, PhCHO (102 $\mu \mathrm{L}$, $1.00 \mathrm{mmol})$ and $(S, S)-1(37 \mathrm{mg}, 0.10 \mathrm{mmol})$ in $1 \mathrm{~mL}$ of $\mathrm{CH}_{2} \mathrm{Cl}_{2}, 203 \mathrm{mg}(0.60 \mathrm{mmol}, 60 \%)$ of 47a was obtained as a clear viscous oil after column chromatography (silica gel, pentane/ether, 3/1). The product was further purified by recrystalization in pentane.

Data for $(s y n, s y n)-47 \mathbf{a}$ :

mp: $\quad 46-48^{\circ} \mathrm{C}$ (pentane)

${ }^{1} \mathrm{H}$ NMR: $\quad\left(500 \mathrm{MHz}, \mathrm{CDCl}_{3}\right)$

7.35-7.31 (m, 4 H, HC(8), HC(9)); 7.27 - 7.25 (m, 1 H, HC(10)); 5.09 (t, J = 3.4, $1 \mathrm{H}, \mathrm{HC}(1)) ; 4.32$ (sext, $J=6.1,1 \mathrm{H}, \mathrm{HC}(5)) ; 3.19$ (d, $J=2.5,1 \mathrm{H}, \mathrm{HO}) ; 2.81$ (qd, $J=7.3,3.7,1 \mathrm{H}, \mathrm{HC}(2)) ; 2.76-2.34\left(\mathrm{ABX}, 2 \mathrm{H}, \mathrm{H}_{2} \mathrm{C}(4)\right) ; 1.12(\mathrm{~d}, J=6.1,3 \mathrm{H}$, 
$\left.\mathrm{H}_{3} \mathrm{C}(6)\right) ; 1.05$ (d, $\left.J=7.1,3 \mathrm{H}, \mathrm{H}_{3} \mathrm{C}(11)\right) ; 0.85$ (s, 9H, $\left.\mathrm{H}_{3} \mathrm{C}(14)\right)$; 0.05 (d, $J=16.8$, $6 \mathrm{H}, \mathrm{H}_{3} \mathrm{C}(12)$ )

${ }^{13} \mathrm{C} \mathrm{NMR}: \quad\left(126 \mathrm{MHz}, \mathrm{CDCl}_{3}\right)$

214.2 (C(3)); 141.7 (C(7)); 128.2 (C(9)); 127.3 (C(10)); 125.9 (C(8)); 72.8 (C(5)); 65.9 (C(1)); 53.3 (C(2)); $51.5(\mathrm{C}(4)) ; 25.7$ (C(14)); 23.8 (C(6)); 17.9 (C(13)); 9.4 $(\mathrm{C}(11)) ;-4.7,-4.9(\mathrm{C}(12))$

IR: (neat)

3487 (br, m); 2956 (m); 2931 (m); 2885 (m); 2858 (m); 1709 (m); 1454 (m); 1408 (w); 1375 (m); $1254(\mathrm{~m}) ; 1154$ (m); 1093 (m)

$\underline{\mathrm{MS}}: \quad(\mathrm{FI})$

$336\left(\mathrm{M}^{+}, 8\right) ; 279$ (100); $230(7) ; 173(10) ; 106(7)$

Opt. Rot.: $\quad[\alpha]_{\mathrm{D}}^{24}+50.3(\mathrm{EtOH}, \mathrm{c}=0.29)$

TLC: $\quad \operatorname{Rf} 0.20$ (pentane/ether, 5/1) [silica gel, $p$-anisaldehyde]

SFC: $\quad t_{R}=3.030 \mathrm{~min}(\mathrm{OD}, 150 \mathrm{bar}, 3 \mathrm{~mL} / \mathrm{min}, 5 \% \mathrm{MeOH})$

Analysis: $\quad \mathrm{C}_{19} \mathrm{H}_{32} \mathrm{O}_{3} \mathrm{Si}(336.54)$

Calcd: $\quad$ C, $67.81 \% ; \quad \mathrm{H}, 9.58 \%$

Found: $\quad$ C, $67.78 \% ; \quad H, 9.61 \%$

$(1 R, 2 R, 5 S)-5$-Triisopropylsilyloxy-2-methyl-1-hydroxy-1-phenyl-3-hexanone (syn,anti-48a)

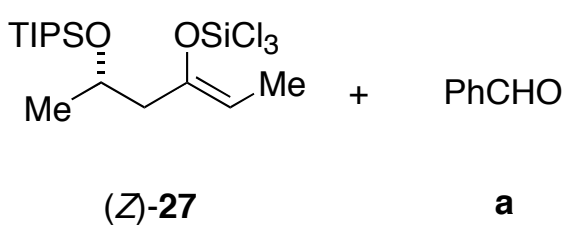

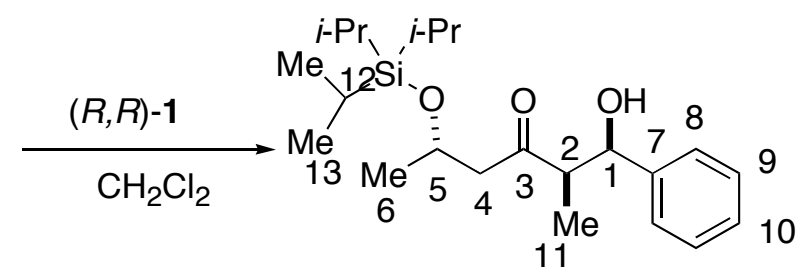

(syn, anti)-48a

Following General Procedure VIII using (Z)-27 (456 mg, $1.00 \mathrm{mmol})$, PhCHO (102 $\mu \mathrm{L}$, $1.00 \mathrm{mmol})$ and $(R, R)-\mathbf{1}(37 \mathrm{mg}, 0.10 \mathrm{mmol})$ in $1 \mathrm{~mL}$ of $\mathrm{CH}_{2} \mathrm{Cl}_{2}$ for $6 \mathrm{~h}, 317 \mathrm{mg}(0.84 \mathrm{mmol}$, $84 \%$ ) of 48a was obtained as a clear viscous oil after column chromatography (silica gel, pentane/ether, 5/1). 


\section{Data for (syn,anti)-48a:}

${ }^{1} \mathrm{H}$ NMR: $\quad\left(500 \mathrm{MHz}, \mathrm{CDCl}_{3}\right)$

7.30 (d, $J=7.6,2 \mathrm{H}, \mathrm{HC}(9)) ; 7.17$ (t, $J=7.3,2 \mathrm{H}, \mathrm{HC}(8)) ; 7.08$ (t, $J=7.2,1 \mathrm{H}$, $\mathrm{HC}(10)) ; 5.04$ (t, $J=3.2,1 \mathrm{H}, \mathrm{HC}(1)) ; 4.44$ (sext, $J=6.1,1 \mathrm{H}, \mathrm{HC}(5)) ; 2.74$ (d, $J$ $=2.6,1 \mathrm{H}, \mathrm{HO}) ; 2.55(\mathrm{qd}, J=7.3,3.9,1 \mathrm{H}, \mathrm{HC}(2)) ; 2.46-2.21(\mathrm{ABX}, 2 \mathrm{H}$, $\left.\mathrm{H}_{2} \mathrm{C}(4)\right) ; 1.11$ (d, J = 6.1, $\left.3 \mathrm{H}, \mathrm{H}_{3} \mathrm{C}(6)\right) ; 1.10$ - 1.02 (m, $\left.21 \mathrm{H}, \mathrm{H}_{3} \mathrm{C}(13), \mathrm{HC}(12)\right)$; $0.94\left(\mathrm{~d}, J=7.0,3 \mathrm{H}, \mathrm{H}_{3} \mathrm{C}(11)\right)$

${ }^{13} \mathrm{C} \mathrm{NMR}: \quad\left(126 \mathrm{MHz}, \mathrm{CDCl}_{3}\right)$

214.8 (C(3)); 141.9 (C(7)); 128.5 (C(8)); 127.6 (C(10)); 126.1 (C(9)); 72.8 (C(5)); $65.4(\mathrm{C}(1)) ; 53.6(\mathrm{C}(2)) ; 52.2(\mathrm{C}(4)) ; 24.3(\mathrm{C}(6)) ; 18.3$ (C(13)); 12.6 (C(12)); 9.8 (C(11))

IR: (neat)

3467 (br, m); 3064 (w); 3032 (w); 2962 (s); 2943 (s); 2893 (s); 2868 (s); 1705 (s); 1462 (s); 1375 (s); 1248 (m); 1213 (m); 1198 (m); 1128(s); 1097 (s); 1070 (m); $1012(\mathrm{~s})$

MS: $\quad(\mathrm{FAB})$

379 (M+, 13); 273 (11); 230 (18); 229 (100); 202 (10); 201 (49); 185 (15); 175 (10); 159 (12); 157 (51); 154 (11), 137 (11); 136 (13); 131 (15); 115 (24); 107 (32); 105 (11), 103 (11)

Opt. Rot.: $\quad[\alpha]_{\mathrm{D}}^{24}-25.9(\mathrm{EtOH}, \mathrm{c}=0.24)$

TLC: $\quad R f 0.17$ (pentane/ether, 5/1) [silica gel, $p$-anisaldehyde]

SFC: $\quad t_{R}=3.645 \mathrm{~min}(\mathrm{OD}, 150 \mathrm{bar}, 3 \mathrm{~mL} / \mathrm{min}, 5 \% \mathrm{MeOH})$

Analysis: $\quad \mathrm{C}_{22} \mathrm{H}_{38} \mathrm{O}_{3} \mathrm{Si}(378.62)$
Calcd:
C, 69.79\%;
$\mathrm{H}, 10.12 \%$

Found:

C, 69.63\%;

$\mathrm{H}, 10.43 \%$ 
(1S, 2S, 5S)-1-Triisopropylsilyloxy-2-methyl-5-hydroxy-5-phenyl-3-hexanone (syn, syn-48a)

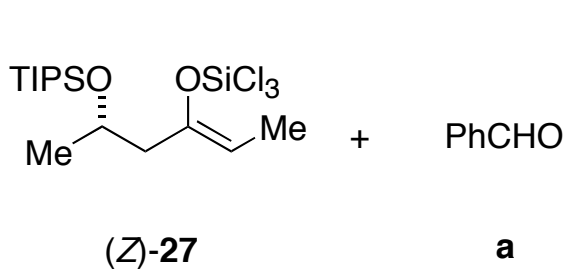
$(Z)-27$

(syn,syn)-48a

Following General Procedure VIII using (Z)-27 (450 mg, $1.00 \mathrm{mmol})$, PhCHO (102 $\mu \mathrm{L}$, $1.00 \mathrm{mmol})$ and $(S, S)-1(37 \mathrm{mg}, 0.10 \mathrm{mmol})$ in $1 \mathrm{~mL}$ of $\mathrm{CH}_{2} \mathrm{Cl}_{2}$ for $6 \mathrm{~h}, 327 \mathrm{mg}(0.86 \mathrm{mmol}$, $86 \%$ ) of 48a was obtained as a clear viscous oil after column chromatography (silica gel, pentane/ether, 5/1).

Data for $(s y n, s y n)-\mathbf{4 8 a}$ :

${ }^{1} \mathrm{H}$ NMR: $\quad\left(500 \mathrm{MHz}, \mathrm{CDCl}_{3}\right)$

7.35 - 7.31 (m, 4 H, HC(9), HC(8)); 7.27 (m, $1 \mathrm{H}, \mathrm{HC}(10)$ ); 5.09 (t, J = 3.4, 1 H, $\mathrm{HC}(1)) ; 4.40$ (sext, $J=5.9,1 \mathrm{H}, \mathrm{HC}(5)) ; 3.05$ (d, $J=2.7,1 \mathrm{H}, \mathrm{HO}) ; 2.85$ (qd, $J=$ 7.3, 3.9, $1 \mathrm{H}, \mathrm{HC}(2)) ; 2.87-2.48\left(\mathrm{ABX}, 2 \mathrm{H}, \mathrm{H}_{2} \mathrm{C}(4)\right) ; 1.15(\mathrm{~d}, J=6.1,3 \mathrm{H}$, $\left.\mathrm{H}_{3} \mathrm{C}(6)\right) ; 1.08-1.00$ (m, $\left.24 \mathrm{H}, \mathrm{H}_{3} \mathrm{C}(13), \mathrm{HC}(12), \mathrm{H}_{3} \mathrm{C}(11)\right)$

${ }^{13} \mathrm{C}$ NMR: $\quad\left(126 \mathrm{MHz}, \mathrm{CDCl}_{3}\right)$ 214.5 (C(3)); 141.9 (C(7)); 128.5 (C(8)); 127.6 (C(10)); 126.2 (C(9)); $73.1(\mathrm{C}(5))$; 65.1 (C(1)); 53.4 (C(2)); 52.3 (C(4)); 24.3 (C(6)); 18.3 (C(13)); 12.6 (C(12)); 10.1 (C(11))

IR: $\quad$ (neat)

3461 (br, m); 2963 (s); 2943 (s); 2893 (s); 2867 (s); 1707 (s); 1461 (m); 1375 (m); 1130 (s); 1097 (m); 1013 (s)

MS: $\quad(\mathrm{FI})$

379 (M+, 4); 337 (8); 336 (30); 335 (100); 272 (6); 229 (13); 157 (4); 157 (4), 157

(5); 157 (6)

Opt. Rot.: $\quad[\alpha]_{\mathrm{D}}^{24}+29.1(\mathrm{EtOH}, \mathrm{c}=0.59)$

TLC: $\quad \operatorname{Rf} 0.17$ (pentane/ether, 5/1) [silica gel, $p$-anisaldehyde]

SFC: $\quad t_{R}=4.452 \mathrm{~min}(\mathrm{OD}, 150 \mathrm{bar}, 3 \mathrm{~mL} / \mathrm{min}, 5 \% \mathrm{MeOH})$ 
Analysis: $\quad \mathrm{C}_{22} \mathrm{H}_{38} \mathrm{O}_{3} \mathrm{Si}(378.62)$

Calcd: $\quad$ C, $69.79 \%$;, $10.12 \%$

Found: $\quad$ C, $69.54 \%$;, $10.32 \%$

$(1 R, 2 R, 5 S)-5$-Triisopropylsilyloxy-2-methyl-1-hydroxy-1-(1-naphthyl)-3-hexanone (syn,anti-48b)

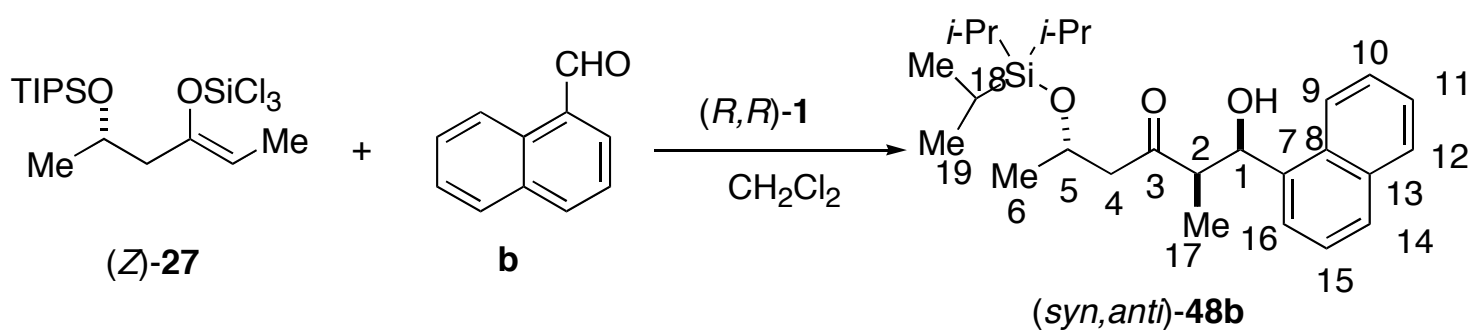

Following General Procedure VIII using (Z)-27 (451 mg, $1.00 \mathrm{mmol}$ ), 1-naphthaldehyde $(136 \mu \mathrm{L}, 1.00 \mathrm{mmol})$ and $(R, R)-\mathbf{1}(37 \mathrm{mg}, 0.10 \mathrm{mmol})$ in $1 \mathrm{~mL}$ of $\mathrm{CH}_{2} \mathrm{Cl}_{2}$ for $8 \mathrm{~h}, 356 \mathrm{mg}(0.83$ mmol, $83 \%)$ of $\mathbf{4 8 b}$ was obtained as a clear viscous oil after column chromatography $(5 / 1$ pentane/ether, 5/1).

Data for (syn,anti)-48b:

${ }^{1} \mathrm{H}$ NMR: $\quad\left(500 \mathrm{MHz}, \mathrm{CDCl}_{3}\right)$

7.89 (d, $J=6.2,1 \mathrm{H}, \mathrm{HC}(9)) ; 7.87$ (d, $J=5.9,1 \mathrm{H}, \mathrm{HC}(12)) ; 7.78$ (d, $J=8.0,1 \mathrm{H}$, $\mathrm{HC}(14)) ; 7.72$ (d, $J=7.3,1 \mathrm{H}, \mathrm{HC}(16)) ; 7.52-7.47$ (m, $3 \mathrm{H}, \mathrm{HC}(11), \mathrm{HC}(10)$, $\mathrm{HC}(15)) ; 5.96$ (t, $J=2.4,1 \mathrm{H}, \mathrm{HC}(1)) ; 4.48$ (sext, $J=6.4,1 \mathrm{H}, \mathrm{HC}(5)) ; 3.26$ (d, $J$ $=2.4,1 \mathrm{H}, \mathrm{HO}) ; 3.03(\mathrm{qd}, J=7.1,4.4,1 \mathrm{H}, \mathrm{HC}(2)) ; 2.81-2.61(\mathrm{ABX}, 2 \mathrm{H}$, $\left.\mathrm{H}_{2} \mathrm{C}(4)\right) ; 1.22\left(\mathrm{~d}, J=5.8, \mathrm{H}_{3} \mathrm{C}(6)\right) ; 1.10-1.04\left(\mathrm{~m}, 24 \mathrm{H}, \mathrm{H}_{3} \mathrm{C}(17), \mathrm{HC}(18)\right.$, $\left.\mathrm{H}_{3} \mathrm{C}(19)\right)$

${ }^{13} \mathrm{C}$ NMR: $\quad\left(126 \mathrm{MHz}, \mathrm{CDCl}_{3}\right)$

214.4 (C(3)); 136.7 (C(7)); 133.7 (C(13)); 129.6 (C(8)); 129.1 (C(12)); 127.8 (C(14)); 126.0 (C(10)); 125.4 (C(11)); 125.3 (C(15)); 124.3 (C(16)); 122.4 (C(9)); 69.1 (C(1)); 64.9 (C(5); 51.9 (C(4)); 51.4 (C(2)); 24.1 (C(6)); 18.0 (C(19)); 12.3 (C(18)); $9.6(\mathrm{C}(17))$ 
IR: $\quad\left(\mathrm{CH}_{2} \mathrm{Cl}_{2}\right)$

3468 (br, m); 2962 (s); 2944 (s); 2892 (s); 2867 (s); 1704 (s); 1462 (s); 1374 (s);

1133 (s); 1102 (s); 1056 (m); 1029 (s); 1013 (s)

MS: $\quad(\mathrm{FI})$

$429\left(\mathrm{M}^{+}, 4\right) ; 411$ (7); 410 (13); 387 (7); 387 (6); 386 (28); 385 (100); 384 (4); 299

(4); 282 (4); 274 (4); 273 (15); 272 (57); 256 (4); 254 (4); 231 (4); 230 (8); 229

(58); 193 (5); 157 (8); $156(44) ; 136(5) ; 136(5)$

Opt. Rot.: $\quad[\alpha]_{\mathrm{D}}^{24}-4.73(\mathrm{EtOH}, \mathrm{c}=0.61)$

TLC: $\quad R f 0.09$ (pentane/ether, 5/1) [silica gel, short uv]

SFC: $\quad t_{R}=5.940 \mathrm{~min}(\mathrm{OD}, 150 \mathrm{bar}, 3 \mathrm{~mL} / \mathrm{min}, 15 \% \mathrm{MeOH})$

Analysis: $\quad \mathrm{C}_{26} \mathrm{H}_{40} \mathrm{O}_{3} \mathrm{Si}(428.68)$

Calcd: $\quad$ C, $72.85 \% ; \quad \mathrm{H}, 9.41 \%$

Found: $\quad$ C, $72.68 \% ; \quad H, 9.49 \%$

(1S, 2S, 5S)-5-Triisopropylsilyloxy-2-methyl-1-hydroxy-1-(1-naphthyl)-3-hexanone (syn,syn48b)<smiles>C/C=C(/C[C@@H](C)O[Na])O[Na]</smiles>

$(Z)-27$<smiles>O=Cc1cccc2ccccc12</smiles>

b

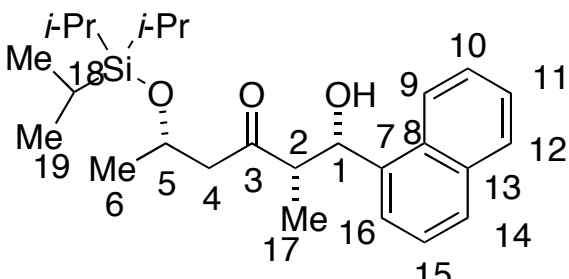

(syn,syn)-48b

Following General Procedure VIII using (Z)-27 (451 mg, $1.00 \mathrm{mmol}$ ), 1-naphthaldehyde $(136 \mu \mathrm{L}, 1.00 \mathrm{mmol})$ and $(S, S)-1(37 \mathrm{mg}, 0.10 \mathrm{mmol})$ in $1 \mathrm{~mL}$ of $\mathrm{CH}_{2} \mathrm{Cl}_{2}$ for $8 \mathrm{~h}, 347 \mathrm{mg}(0.75$ $\mathrm{mmol}, \mathbf{7 5 \%}$ ) of $\mathbf{4 8 b}$ was obtained as a clear viscous oil after column chromatography (silica gel, pentane/ether, 5/1).

Data for $(s y n, s y n)-\mathbf{4 8 b}$ :

${ }^{1} \mathrm{H}$ NMR: $\quad\left(500 \mathrm{MHz}, \mathrm{CDCl}_{3}\right)$

7.88 (d, $J=7.4,1 \mathrm{H}, \mathrm{HC}(9)) ; 7.87$ (d, $J=4.1,1 \mathrm{H}, \mathrm{HC}(12)) ; 7.79$ (d, $J=8.0,1 \mathrm{H}$, $\mathrm{HC}(14)) ; 7.72(\mathrm{~d}, J=7.1,1 \mathrm{H}, \mathrm{HC}(16)) ; 7.52-7.48$ (m, $3 \mathrm{H}, \mathrm{HC}(11), \mathrm{HC}(10)$, 
$\mathrm{HC}(15)) ; 5.94$ (dd, $J=3.4,2.5,1 \mathrm{H}, \mathrm{HC}(1)) ; 4.46$ (sext, $J=6.1,1 \mathrm{H}, \mathrm{HC}(5)$ ); 3.24 (d, $J=2.4,1 \mathrm{H}, \mathrm{HO}) ; 3.04$ (qd, $J=7.3,4.4,1 \mathrm{H}, \mathrm{HC}(2)) ; 2.87$ - 2.57 (ABX, $2 \mathrm{H}$, $\left.\mathrm{H}_{2} \mathrm{C}(4)\right) ; 1.21\left(\mathrm{~d}, J=6.1, \mathrm{H}_{3} \mathrm{C}(6)\right) ; 1.09-1.02$ (m, $24 \mathrm{H}, \mathrm{H}_{3} \mathrm{C}(17), \mathrm{HC}(18)$, $\left.\mathrm{H}_{3} \mathrm{C}(19)\right)$

${ }^{13} \mathrm{C} \mathrm{NMR}: \quad\left(126 \mathrm{MHz}, \mathrm{CDCl}_{3}\right)$

214.3 (C(3)); 136.6 (C(7)); 133.7 (C(13)); 129.6 (C(8)); 129.1 (C(12)); 127.9 (C(14)); 126.1 (C(10)); 125.4 (C(11)); 125.3 (C(15)); 124.1 (C(16)); 122.3 (C(9)); 69.2 (C(1)); 64.9 (C(5)); 51.8 (C(4)); $51.4(\mathrm{C}(2)) ; 24.1$ (C(6)); 18.0 (C(19)); 12.3 (C(18)); $9.6(\mathrm{C}(17))$

IR: (neat)

3470 (br, m); 2963 (s); 2943 (s); 2892 (m); 2867 (s); 1704 (m); 1462 (m); 1374 (m); $1130(\mathrm{~m}) ; 1104(\mathrm{~m}) ; 1013(\mathrm{~s})$

MS: $\quad(\mathrm{FI})$

$429\left(\mathrm{M}^{+}, 16\right) ; 428$ (10); 386 (28); 385 (95); 321 (17); 303 (11); 273 (21); 272 (58); 230 (17); 229 (97); 157 (20); 156 (100)

Opt. Rot.: $\quad[\alpha]_{\mathrm{D}}^{24}+14.2(\mathrm{EtOH}, \mathrm{c}=0.63)$

TLC: $\quad R f 0.09$ (pentane/ether, 5/1) [silica gel, short uv]

SFC: $\quad t_{R}=3.562 \mathrm{~min}(\mathrm{OD}, 150 \mathrm{bar}, 3 \mathrm{~mL} / \mathrm{min}, 15 \% \mathrm{MeOH})$

Analysis: $\quad \mathrm{C}_{26} \mathrm{H}_{40} \mathrm{O}_{3} \mathrm{Si}(428.68)$
Calcd:
C, $72.85 \%$;
$\mathrm{H}, 9.41 \%$
Found:
C, $73.02 \%$;
$\mathrm{H}, 9.78 \%$ 
$(2 S, 5 R, 6 R)$-2-Triisopropylsilyloxy-5-methyl-6-hydroxy-8-phenyl-7-octen-4-one (syn,anti-

48c)

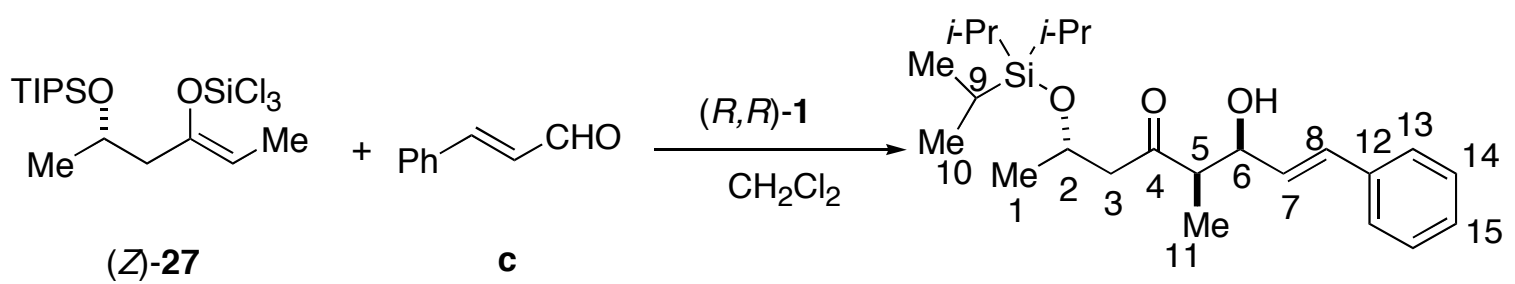

(syn, anti)-48c

Following General Procedure VIII using (Z)-27 (451 mg, $1.00 \mathrm{mmol})$, cinnamaldehyde $(126 \mu \mathrm{L}, 1.00 \mathrm{mmol})$ and $(R, R)-\mathbf{1}(37 \mathrm{mg}, 0.10 \mathrm{mmol})$ in $1 \mathrm{~mL}$ of $\mathrm{CH}_{2} \mathrm{Cl}_{2}$ for $12 \mathrm{~h}, 322 \mathrm{mg}(0.80$ mmol, $80 \%$ ) of $48 \mathrm{c}$ was obtained as a clear viscous oil after column chromatography (silica gel, pentane/ether, 2/1).

Data for (syn,anti)-48c:

${ }^{1} \mathrm{H}$ NMR: $\quad\left(500 \mathrm{MHz}, \mathrm{CDCl}_{3}\right)$

$7.37(\mathrm{~d}, J=7.3,2 \mathrm{H}, \mathrm{HC}(13)) ; 7.31(\mathrm{t}, J=7.6,2 \mathrm{H}, \mathrm{HC}(14)) ; 7.24$ (t, $J=7.1,1$ H, HC(15)); 6.64 (d, $J=15.9,1 \mathrm{H}, \mathrm{HC}(8)) ; 6.14$ (dd, $J=15.8,5.9,1 \mathrm{H}, \mathrm{HC}(7)$ ); 4.67 (m, $1 \mathrm{H}, \mathrm{HC}(6))$ ) 4.45 (sext, $J=6.1,1 \mathrm{H}, \mathrm{HC}(2)) ; 2.88$ (d, J= 3.2, $1 \mathrm{H}, \mathrm{HO}$ ); 2.82-2.59 (ABX, $\left.2 \mathrm{H}, \mathrm{H}_{2} \mathrm{C}(3)\right) ; 2.76$ (qd, $\left.J=7.3,3.4,1 \mathrm{H}, \mathrm{HC}(5)\right) ; 1.22$ (d, $J$ $\left.=6.1,3 \mathrm{H}, \mathrm{H}_{3} \mathrm{C}(1)\right) ; 1.17\left(\mathrm{~d}, J=7.1,3 \mathrm{H}, \mathrm{H}_{3} \mathrm{C}(11)\right) ; 1.05(\mathrm{~m}, 21 \mathrm{H}, \mathrm{HC}(9)$, $\left.\mathrm{H}_{3} \mathrm{C}(10)\right)$

${ }^{13} \mathrm{C}$ NMR: $\quad\left(126 \mathrm{MHz}, \mathrm{CDCl}_{3}\right)$

219.3 (C(4)); $136.6(\mathrm{C}(12)) ; 131.1(\mathrm{C}(7)) ; 128.9$ (C(8)); 128.6 (C(14)); 127.7

(C(15)); 126.5 (C(13)); 71.8 (C(6)); 65.3 (C(2)); $52.0(\mathrm{C}(3)) ; 51.5(\mathrm{C}(5)) ; 24.2$ $(\mathrm{C}(1)) ; 18.1(\mathrm{C}(10)) ; 12.4(\mathrm{C}(9)) ; 10.0(\mathrm{C}(11))$

IR: (neat)

3442 (br, m); 2963 (s); 2943 (s); 2892 (s); 2867 (s); 1705 (s); 1462 (m); 1374 (m); $1126(\mathrm{~s}) ; 1013$ (s)

$\underline{\mathrm{MS}}: \quad(\mathrm{FI})$

$404\left(\mathrm{M}^{+}, 9\right) ; 361$ (7); 321 (2); 272 (13); 229 (100); 132 (86)

Opt. Rot:: $\quad[\alpha]_{D}^{24}-20.5(\mathrm{EtOH}, \mathrm{c}=0.33)$ 
TLC: $\quad R f 0.21$ (pentane/ether, 2/1) [silica gel, short uv]

SFC: $\quad t_{R}=2.348 \mathrm{~min}(\mathrm{OD}, 150 \mathrm{bar}, 3 \mathrm{~mL} / \mathrm{min}, 15 \% \mathrm{MeOH})$

Analysis: $\quad \mathrm{C}_{24} \mathrm{H}_{40} \mathrm{O}_{3} \mathrm{Si}(404.66)$

Calcd: $\quad$ C, $71.23 \% ; \quad H, 9.96 \%$

Found: $\quad$ C, $71.20 \% ; \quad H, 10.21 \%$

$(2 S, 5 S, 6 S)$-2-Triisopropylsilyloxy-5-methyl-6-hydroxy-8-phenyl-7-octen-4-one (syn,syn-

48c)

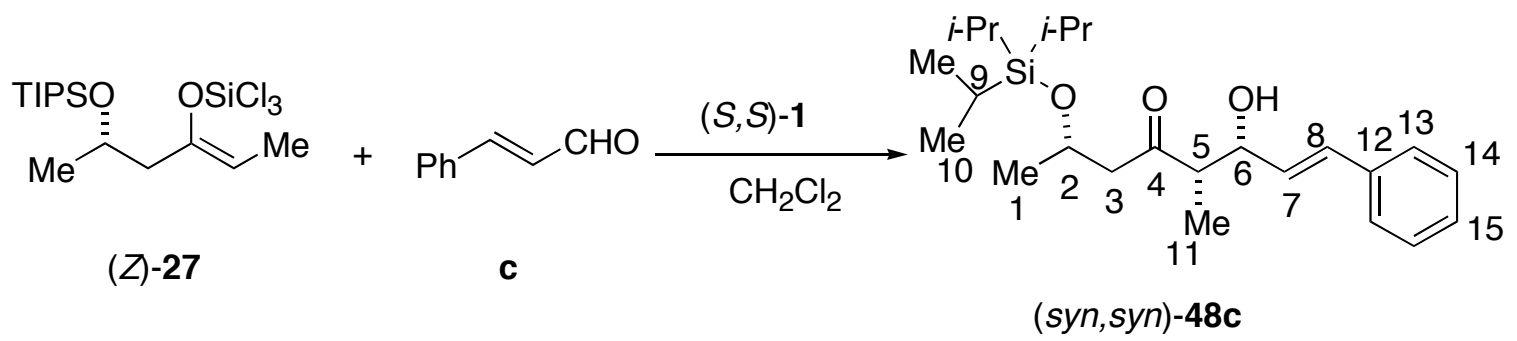

Following General Procedure VIII using (Z)-27 (451 mg, $1.00 \mathrm{mmol})$, cinnamaldehyde $(126 \mu \mathrm{L}, 1.00 \mathrm{mmol})$ and $(S, S)-\mathbf{1}(37 \mathrm{mg}, 0.10 \mathrm{mmol})$ in $1 \mathrm{~mL}$ of $\mathrm{CH}_{2} \mathrm{Cl}_{2}$ for $12 \mathrm{~h}, 304 \mathrm{mg}(0.75$ mmol, $75 \%$ ) of $48 \mathrm{c}$ was obtained as a clear viscous oil after column chromatography (silica gel, pentane/ether, 2/1).

Data for (syn,syn)-48c:

${ }^{1}$ H NMR: $\quad\left(500 \mathrm{MHz}, \mathrm{CDCl}_{3}\right)$

7.37 (d, $J=7.1,2 \mathrm{H}, \mathrm{HC}(13)) ; 7.31$ (t, $J=7.8,2 \mathrm{H}, \mathrm{HC}(14)) ; 7.24$ (t, $J=7.4,1$ $\mathrm{H}, \mathrm{HC}(15)) ; 6.64(\mathrm{dd}, J=15.9,1.2,1 \mathrm{H}, \mathrm{HC}(8)) ; 6.15(\mathrm{dd}, J=15.8,5.8,1 \mathrm{H}$, $\mathrm{HC}(7)) ; 4.66$ (m, $1 \mathrm{H}, \mathrm{HC}(6)) ; 4.45$ (sext, $J=5.6,1 \mathrm{H}, \mathrm{HC}(2)) ; 2.85$ (d, $J=3.2,1$ $\mathrm{H}, \mathrm{HO}$ ); 2.78 (m, $1 \mathrm{H}, \mathrm{HC}(5)) ; 2.80-2.62\left(\mathrm{ABX}, 2 \mathrm{H}, \mathrm{H}_{2} \mathrm{C}(3)\right) ; 1.22$ (d, $J=6.1,3$ $\left.\mathrm{H}, \mathrm{H}_{3} \mathrm{C}(1)\right) ; 1.18\left(\mathrm{~d}, J=7.3,3 \mathrm{H}, \mathrm{H}_{3} \mathrm{C}(11)\right) ; 1.08-1.03(\mathrm{~m}, 21 \mathrm{H}, \mathrm{HC}(9)$, $\left.\mathrm{H}_{3} \mathrm{C}(10)\right)$

${ }^{13} \mathrm{C} \mathrm{NMR}: \quad\left(126 \mathrm{MHz}, \mathrm{CDCl}_{3}\right)$

214.0 (C(4)); $136.6(\mathrm{C}(12)) ; 131.1(\mathrm{C}(7)) ; 129.0(\mathrm{C}(8)) ; 128.6(\mathrm{C}(14)) ; 127.7$ $(\mathrm{C}(15)) ; 126.5(\mathrm{C}(13)) ; 72.0(\mathrm{C}(6)) ; 65.1(\mathrm{C}(2)) ; 52.0(\mathrm{C}(3)) ; 51.4(\mathrm{C}(5)) ; 24.1$ $(\mathrm{C}(1)) ; 18.1(\mathrm{C}(10)) ; 12.4(\mathrm{C}(9)) ; 10.2(\mathrm{C}(11))$ 
IR: (neat)

3436 (br, m); 2963 (s); 2943 (s); 2892 (m); 2867 (s); 1707 (m); 1462 (m); 1374 (m); $1130(\mathrm{~m}) ; 1013(\mathrm{~s})$

MS: $\quad(\mathrm{FI})$

$404\left(\mathrm{M}^{+}, 18\right) ; 385$ (8); 361 (7); 321 (9); 273 (10); 272 (21); 245 (9); 230 (19); 229

(100); $156(8) ; 133$ (12); 132 (74)

Opt. Rot.: $\quad[\alpha]_{\mathrm{D}}^{24}+27.7(\mathrm{EtOH}, \mathrm{c}=0.50)$

TLC: $\quad$ Rf 0.20 (pentane/ether, 2/1) [silica gel, short uv]

SFC: $\quad t_{R}=3.231 \mathrm{~min}(\mathrm{OD}, 150 \mathrm{bar}, 3 \mathrm{~mL} / \mathrm{min}, 15 \% \mathrm{MeOH})$

Analysis: $\quad \mathrm{C}_{24} \mathrm{H}_{40} \mathrm{O}_{3} \mathrm{Si}(404.66)$

Calcd: $\quad$ C, $71.23 \% ; \quad H, 9.96 \%$

Found: $\quad$ C, $71.29 \%$; $\quad$ H, $10.31 \%$

\section{$(2 S, 5 R, 6 R)$-2-Triisopropylsilyloxy-11,12-dimethyl-6-hydroxy-8-phenyl-7-octen-4-one} (syn,anti-48d)

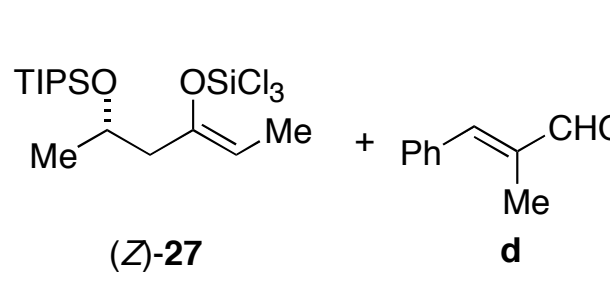

Following General Procedure VIII using (Z)-27 (436 mg, $1.00 \mathrm{mmol})$, $\alpha$ methylcinnamaldehyde $(140 \mu \mathrm{L}, 1.00 \mathrm{mmol})$ and $(R, R)-\mathbf{1}(37 \mathrm{mg}, 0.10 \mathrm{mmol})$ in $1 \mathrm{~mL}$ of $\mathrm{CH}_{2} \mathrm{Cl}_{2}$ for $10 \mathrm{~h}, 332 \mathrm{mg}(0.79 \mathrm{mmol}, 79 \%)$ of $\mathbf{4 8 d}$ was obtained as a clear viscous oil after column chromatography (silica gel, pentane/ether, 3/1).

\section{Data for (syn,anti)-48d:}

${ }^{1}$ H NMR: $\quad\left(500 \mathrm{MHz}, \mathrm{CDCl}_{3}\right)$

7.33 (t, $J=7.8,2 \mathrm{H}, \mathrm{HC}(15)) ; 7.27$ (d, $J=7.3,2 \mathrm{H}, \mathrm{HC}(14)) ; 7.21$ (t, $J=7.0,1$ $\mathrm{H}, \mathrm{HC}(16)$ ); 6.63 (br s, $1 \mathrm{H}, \mathrm{HC}(8)$ ); 4.53 (m, $1 \mathrm{H}, \mathrm{HC}(6)$ ); 4.47 (sext, $J=6.3,1$ $\mathrm{H}, \mathrm{HC}(2)) ; 2.99$ (d, $J=2.7,1 \mathrm{H}, \mathrm{HO}) ; 2.84-2.62\left(\mathrm{ABX}, 2 \mathrm{H}, \mathrm{H}_{2} \mathrm{C}(3)\right) ; 2.82$ (m, 1 
$\mathrm{H}, \mathrm{HC}(5)) ; 1.81\left(\mathrm{~d}, J=1.0,3 \mathrm{H}, \mathrm{H}_{3} \mathrm{C}(12)\right) ; 1.22\left(\mathrm{~d}, J=5.9,3 \mathrm{H}, \mathrm{H}_{3} \mathrm{C}(1)\right) ; 1.13$ (d, $\left.J=7.3,3 \mathrm{H}, \mathrm{H}_{3} \mathrm{C}(11)\right) ; 1.07-1.00\left(\mathrm{~m}, 21 \mathrm{H}, \mathrm{HC}(9), \mathrm{H}_{3} \mathrm{C}(10)\right)$

${ }^{13} \mathrm{C} \mathrm{NMR}: \quad\left(126 \mathrm{MHz}, \mathrm{CDCl}_{3}\right)$

214.3 (C(4)); 137.6 (C(13)); 136.3 (C(7)); 129.0 (C(15)); 128.1 (C(14)); 126.4 (C(16)); 125.9 (C(8)); $74.8(\mathrm{C}(6)) ; 65.3(\mathrm{C}(2)) ; 51.7(\mathrm{C}(3)) ; 49.2(\mathrm{C}(5)) ; 24.1$ (C(1)); 18.0 (C(10)); 15.3 (C(12)); 12.4 (C(9)); 9.3 (C(11))

IR: $\quad\left(\mathrm{CHCl}_{3}\right)$

3487 (br, w); 2962 (m); 2945 (m); 2868 (m); 1699 (w); 1464 (w); 1377 (w); 1101 (w)

$\underline{\mathrm{MS}}: \quad(\mathrm{FI})$

$418\left(\mathrm{M}^{+}, 100\right) ; 375$ (25); 336 (8); 272 (22); 229 (27); 187 (4); 146 (29); 115 (2)

Opt. Rot.: $\quad[\alpha]_{\mathrm{D}}^{24}-2.13(\mathrm{EtOH}, \mathrm{c}=0.63)$

TLC: $\quad R f 0.22$ (pentane/ether, 3/1) [silica gel, short uv]

SFC: $\quad t_{R}=2.03 \mathrm{~min}(\mathrm{OD}, 150 \mathrm{bar}, 3 \mathrm{~mL} / \mathrm{min}, 15 \% \mathrm{MeOH})$

Analysis: $\quad \mathrm{C}_{25} \mathrm{H}_{42} \mathrm{O}_{3} \mathrm{Si}(418.68)$

Calcd: $\quad$ C, $71.72 \%$; $\quad$ H, $10.11 \%$

Found: $\quad$ C, $71.34 \%$; $\quad$ H, $10.18 \%$

\section{$(2 S, 5 S, 6 S)$-2-Triisopropylsilyloxy-11,12-dimethyl-6-hydroxy-8-phenyl-7-octen-4-one (syn,syn-48d)}

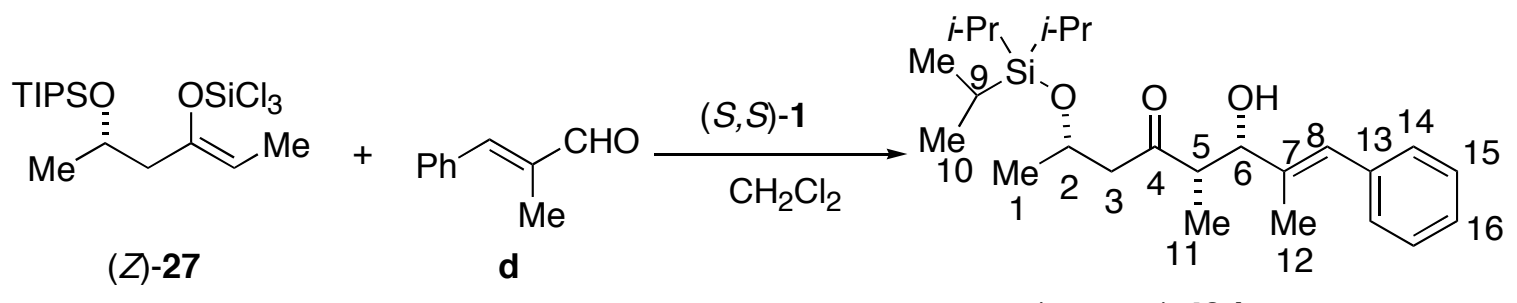

(syn,syn)-48d

Following General Procedure VIII using (Z)-27 (436 mg, $1.00 \mathrm{mmol})$, $\alpha$ methylcinnamaldehyde $(140 \mu \mathrm{L}, 1.00 \mathrm{mmol})$ and $(S, S)-\mathbf{1}(37 \mathrm{mg}, 0.10 \mathrm{mmol})$ in $1 \mathrm{~mL}$ of $\mathrm{CH}_{2} \mathrm{Cl}_{2}$ for $10 \mathrm{~h}, 344 \mathrm{mg}(0.82 \mathrm{mmol}, 82 \%)$ of $\mathbf{4 8 d}$ was obtained as a clear viscous oil after column chromatography (silica gel, pentane/ether, 3/1). 


\section{Data for $(s y n, s y n)-\mathbf{4 8 d}$ :}

${ }^{1}$ H NMR: $\quad\left(500 \mathrm{MHz}, \mathrm{CDCl}_{3}\right)$

$7.33(\mathrm{t}, J=7.3,2 \mathrm{H}, \mathrm{HC}(15)) ; 7.27(\mathrm{~d}, J=6.6,2 \mathrm{H}, \mathrm{HC}(14)) ; 7.21(\mathrm{t}, J=7.4,1$ H, HC(16)); 6.62 (br s, 1 H, HC(8)); 4.52 (br s, 1 H, HC(6)); 4.45 (sext, J = 5.9, 1 H, HC(2)); 2.91 (d, $J=2.9,1 \mathrm{H}, \mathrm{HO}$ ); 2.86 (qd, $J=7.3,3.6,1 \mathrm{H}, \mathrm{HC}(5)$ ); 2.84$2.62\left(\mathrm{ABX}, 2 \mathrm{H}, \mathrm{H}_{2} \mathrm{C}(3)\right) ; 1.83$ (d, $\left.J=0.7,3 \mathrm{H}, \mathrm{H}_{3} \mathrm{C}(12)\right) ; 1.23$ (d, $J=6.1,3 \mathrm{H}$, $\left.\mathrm{H}_{3} \mathrm{C}(1)\right) ; 1.13\left(\mathrm{~d}, J=7.3,3 \mathrm{H}, \mathrm{H}_{3} \mathrm{C}(11)\right) ; 1.06\left(\mathrm{~m}, 21 \mathrm{H}, \mathrm{HC}(9), \mathrm{H}_{3} \mathrm{C}(10)\right)$

${ }^{13} \mathrm{C} \mathrm{NMR}: \quad\left(126 \mathrm{MHz}, \mathrm{CDCl}_{3}\right)$

214.0 (C(4)); 137.6 (C(13)); 136.4 (C(7)); 129.0 (C(15)); 128.1 (C(14)); 126.4 $(\mathrm{C}(16)) ; 126.0(\mathrm{C}(8)) ; 75.0(\mathrm{C}(6)) ; 65.1(\mathrm{C}(2)) ; 51.8(\mathrm{C}(3)) ; 49.0(\mathrm{C}(5)) ; 24.1$ $(\mathrm{C}(1)) ; 18.0(\mathrm{C}(10)) ; 15.3(\mathrm{C}(12)) ; 12.4(\mathrm{C}(9)) ; 9.4(\mathrm{C}(11))$

IR: (neat)

3467 (br, m); 2943 (s); 2866 (s); 1705 (m); 1462 (m); 1375 (m); 1130 (m); 1097 (m); $1012(\mathrm{~m})$

MS: $\quad(\mathrm{FI})$

$418\left(\mathrm{M}^{+}, 55\right) ; 401$ (3); 375. (14); 337 (4); 272 (95); 229 (100); 201 (5); 146 (99)

Opt. Rot.: $\quad[\alpha]_{\mathrm{D}}^{24}+15.1(\mathrm{EtOH}, \mathrm{c}=2.2)$

TLC: $\quad R f 0.22$ (pentane/ether, 3/1) [silica gel, short uv]

SFC: $\quad t_{R}=2.983 \mathrm{~min}(\mathrm{OD}, 150 \mathrm{bar}, 3 \mathrm{~mL} / \mathrm{min}, 15 \% \mathrm{MeOH})$

Analysis: $\quad \mathrm{C}_{25} \mathrm{H}_{42} \mathrm{O}_{3} \mathrm{Si}(418.68)$
Calcd:
C, $71.72 \%$;
$\mathrm{H}, 10.11 \%$

Found:

C, $71.46 \%$;

$\mathrm{H}, 10.04 \%$ 


\section{$(2 S, 5 R, 6 R)$-2-Triisopropylsilyloxy-5-methyl-6-hydroxy-7-nonen-4-one (syn,anti-48e)}

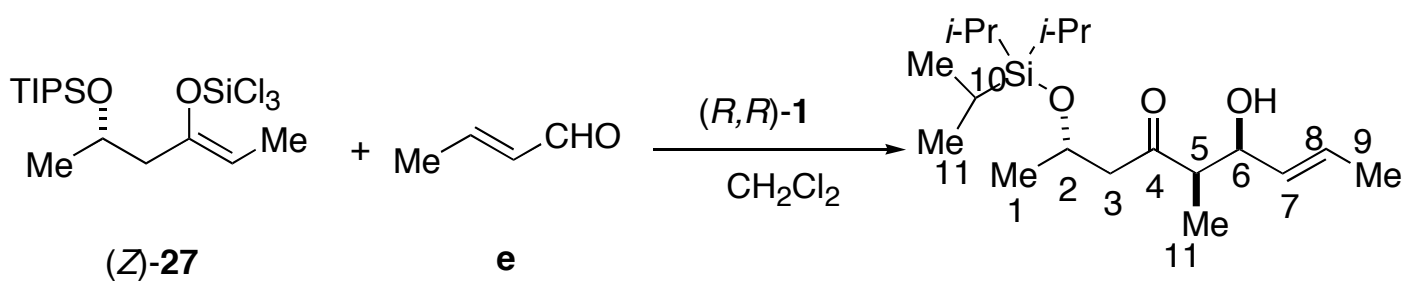

$($ syn, anti) $-\mathbf{4 8 e}$

Following General Procedure VIII using (Z)-27 (451 mg, $1.00 \mathrm{mmol})$, crotonaldehyde $(83 \mu \mathrm{L}, 1.00 \mathrm{mmol})$ and $(R, R)-\mathbf{1}(37 \mathrm{mg}, 0.10 \mathrm{mmol})$ in $1 \mathrm{~mL}$ of $\mathrm{CH}_{2} \mathrm{Cl}_{2}$ for $8 \mathrm{~h}, 272 \mathrm{mg}(0.79$ mmol, 79\%) of 48e was obtained as a clear viscous oil after column chromatography (silica gel, pentane/ether, 3/1).

Data for (syn,anti)-48e:

${ }^{1} \mathrm{H}$ NMR: $\quad\left(500 \mathrm{MHz}\right.$, Benzene- $\left.d_{6}\right)$

$5.61(\mathrm{dqd}, J=15.2,6.4,1.2,1 \mathrm{H}, \mathrm{HC}(8)) ; 5.37(\mathrm{ddd}, J=15.4,6.1,1.5,1 \mathrm{H}$, $\mathrm{HC}(7)$ ); 4.51 (sext, $J=6.3,1 \mathrm{H}, \mathrm{HC}(2)) ; 4.31$ (m, $1 \mathrm{H}, \mathrm{HC}(6)$ ); 2.68 - 2.34 (ABX, $\left.2 \mathrm{H}, \mathrm{H}_{2} \mathrm{C}(3)\right) ; 2.36(\mathrm{~m}, 1 \mathrm{H}, \mathrm{HC}(5)) ; 2.10(\mathrm{~d}, J=3.5,1 \mathrm{H}, \mathrm{HO}) ; 1.53$ (d, $J=6.5,3$ $\left.\mathrm{H}, \mathrm{H}_{3} \mathrm{C}(9)\right) ; 1.17$ (d, $\left.J=6.1,3 \mathrm{H}, \mathrm{H}_{3} \mathrm{C}(1)\right) ; 1.15$ - 1.03 (m, $21 \mathrm{H}, \mathrm{H}_{3} \mathrm{C}(11)$, $\mathrm{HC}(10)) ; 1.01\left(\mathrm{~d}, J=7.3,3 \mathrm{H}, \mathrm{H}_{3} \mathrm{C}(12)\right)$

${ }^{13} \mathrm{C} \mathrm{NMR}: \quad\left(126 \mathrm{MHz}, \mathrm{CDCl}_{3}\right)$

$214.1(\mathrm{C}(4)) ; 130.5(\mathrm{C}(7)) ; 127.9(\mathrm{C}(8) ; 72.2(\mathrm{C}(6)) ; 65.2(\mathrm{C}(2)) ; 52.1(\mathrm{C}(3))$; $51.6(\mathrm{C}(5)) ; 24.1$ (C(1)); 18.0 (C(11)); 17.7 (C(9)); 12.3 (C(10)); 10.2 (C(12))

IR: $\quad$ (neat) 3442 (br, m); 2963 (s); 2943 (s); 2893 (s); 2868 (s); 1710 (s); 1463 (m); 1375 (m); $1130(\mathrm{~s}) ; 1102(\mathrm{~m}) ; 1013(\mathrm{~s})$

$\underline{\mathrm{MS}}: \quad(\mathrm{FI})$

$343\left(\mathrm{M}^{+}, 3\right) ; 301$ (7); 300 (22); 300 (4); 299 (100); 281 (4); 272 (8); 230 (6); 29.1 (36); 176 (3); 176 (4); 176 (4); 176 (5); 176 (6); 176 (6); 175 (5); 175 (5); 175 (4)

Opt. Rot.: $\quad[\alpha]_{\mathrm{D}}^{24}-15.9(\mathrm{EtOH}, \mathrm{c}=0.54)$

TLC: $\quad R f 0.16$ (pentane/ether, 3/1) [silica gel, $p$-anisaldehyde] 
Analysis: $\quad \mathrm{C}_{19} \mathrm{H}_{38} \mathrm{O}_{3} \mathrm{Si}(342.59)$

$\begin{array}{lll}\text { Calcd: } & \text { C, 66.61\%; } & \text { H, } 11.18 \% \\ \text { Found: } & \text { C }, 66.37 \% ; & \text { H }, 11.36 \%\end{array}$

$(2 S, 5 S, 6 S)$-2-Triisopropylsilyloxy-5-methyl-6-hydroxy-7-nonen-4-one (syn,syn-48e)

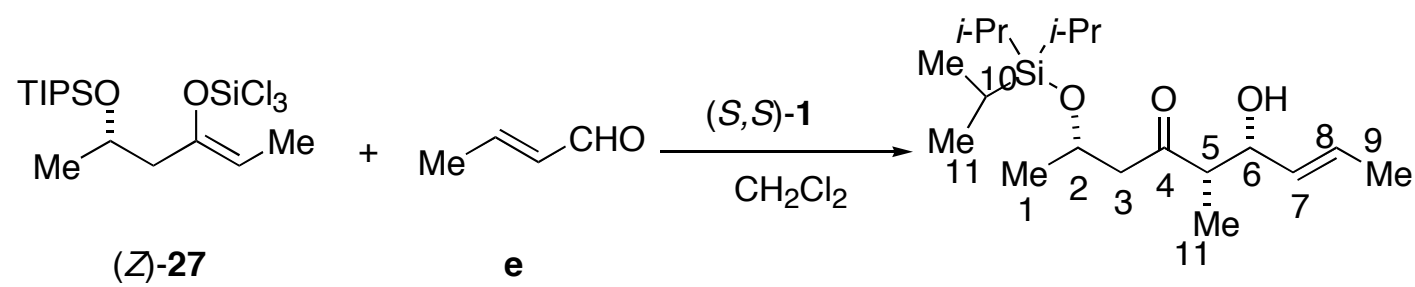

(syn,syn)-48e

Following General Procedure VIII using (Z)-27 (451 mg, $1.00 \mathrm{mmol})$, crotonaldehyde $(83 \mu \mathrm{L}, 1.00 \mathrm{mmol})$ and $(S, S)-1(37 \mathrm{mg}, 0.10 \mathrm{mmol})$ in $1 \mathrm{~mL}$ of $\mathrm{CH}_{2} \mathrm{Cl}_{2}$ for $8 \mathrm{~h}, 285 \mathrm{mg}(0.83$ mmol, $83 \%$ ) of $48 \mathbf{e}$ was obtained as a clear viscous oil after column chromatography (silica gel, pentane/ether, 3/1).

Data for $(s y n, s y n)-48 \mathrm{e}$ :

${ }^{1} \mathrm{H}$ NMR: $\quad\left(500 \mathrm{MHz}\right.$, benzene- $\left.d_{6}\right)$ $5.58(\mathrm{dqd}, J=15.4,6.4,1.2,1 \mathrm{H}, \mathrm{HC}(8)) ; 5.35$ (ddd, $J=15.4,6.1,1.7,1 \mathrm{H}$, $\mathrm{HC}(7)$ ); 4.51 (sext, $J=5.9,1 \mathrm{H}, \mathrm{HC}(2)) ; 4.25$ (m, $1 \mathrm{H}, \mathrm{HC}(6)$ ); 2.68 - 2.34 (ABX, $\left.2 \mathrm{H}, \mathrm{H}_{2} \mathrm{C}(3)\right) ; 2.38(\mathrm{~m}, 1 \mathrm{H}, \mathrm{HC}(5)) ; 2.00$ (d, $\left.J=3.4,1 \mathrm{H}, \mathrm{HO}\right) ; 1.51$ (dd, $J=6.4$, $\left.1.0,3 \mathrm{H}, \mathrm{H}_{3} \mathrm{C}(9)\right) ; 1.18$ (d, $\left.J=6.1,3 \mathrm{H}, \mathrm{H}_{3} \mathrm{C}(1)\right)$; 1.16 - 1.00 (m, $21 \mathrm{H}, \mathrm{H}_{3} \mathrm{C}(11)$, $\mathrm{HC}(10)) ; 1.05$ (d, $\left.J=7.1,3 \mathrm{H}, \mathrm{H}_{3} \mathrm{C}(12)\right)$

${ }^{13} \mathrm{C} \mathrm{NMR}: \quad\left(126 \mathrm{MHz}, \mathrm{CDCl}_{3}\right)$

213.7 (C(4)); $130.6(\mathrm{C}(7)) ; 128.0$ (C(8); $72.4(\mathrm{C}(6)) ; 65.0(\mathrm{C}(2)) ; 52.2$ (C(3)); $51.6(\mathrm{C}(5)) ; 24.0(\mathrm{C}(1)) ; 18.0(\mathrm{C}(11)) ; 17.7$ (C(9)); 12.3 (C(11)); 10.4 (C(12))

IR: (neat) 3441 (br, m); 2963 (s); 2944 (m); 2893 (m); 2867 (s); 1710 (m); 1462 (m); 1375 (m); $1132(\mathrm{~m}) ; 1109(\mathrm{~m}) ; 1013(\mathrm{~m})$

$\underline{\mathrm{MS}}: \quad(\mathrm{FI})$

343 (2); $342\left(\mathrm{M}^{+}, 3\right) ; 301(7) ; 300(22) ; 299$ (100); 229 (37); 175 (5) 
Opt. Rot.: $\quad[\alpha]_{\mathrm{D}}^{24}+30.4(\mathrm{EtOH}, \mathrm{c}=0.54)$

TLC: $\quad R f 0.16$ (pentane/ether, 3/1) [silica gel, $p$-anisaldehyde]

Analysis: $\quad \mathrm{C}_{19} \mathrm{H}_{38} \mathrm{O}_{3} \mathrm{Si}(342.59)$
Calcd:
C, $66.61 \%$
$\mathrm{H}, 11.18 \%$
Found:
C, 66.33\%;
$\mathrm{H}, 11.33 \%$

\section{General Procedure IX: Aldol Addition of TMS Enol ether 25: (1R,2S,4S)-1-Hydroxy-2,4- dimethyl-1-phenyl-5-triisopropylsilanyloxypentan-3-one (anti,anti-46a)}

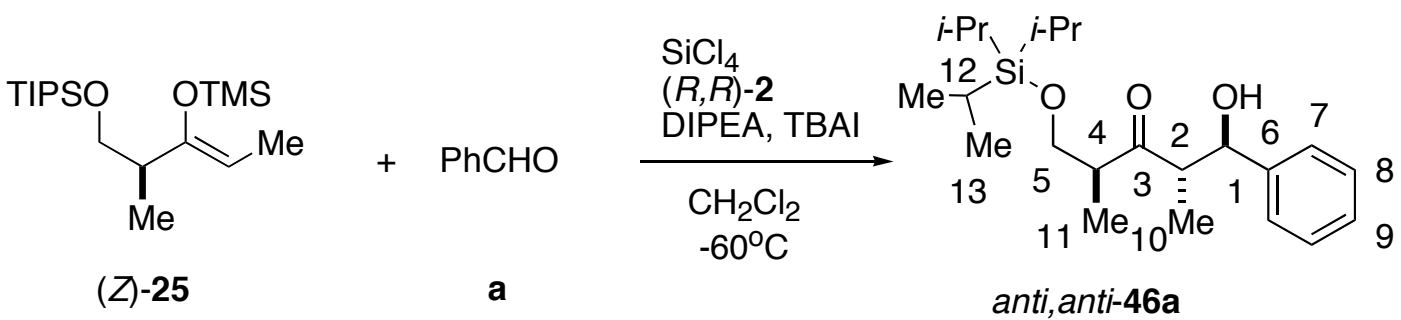

In a-10 mL Schlenk flask were placed $(R, R)-2$ (84 mg, $0.10 \mathrm{mmol}, 0.10$ equiv), tetrabutylammonium iodide ( $74 \mathrm{mg}, 0.20 \mathrm{mmol}, 0.2$ equiv) in dichloromethane $(2 \mathrm{~mL})$. To the solution were added benzaldehyde $(0.152 \mathrm{~mL}, 1.50 \mathrm{mmol}, 1.5$ equiv) and diisopropylethylamine ( $0.035 \mathrm{~mL}, 0.20 \mathrm{mmol}, 02$ equiv), and the solution was cooled to $-72{ }^{\circ} \mathrm{C}$ (internal temperature) using a dry ice/isopropanol bath. To the solution was added silicon tetrachloride $(0.172 \mathrm{~mL}$, $1.50 \mathrm{mmol}, 1.5$ equiv), and the solution was stirred for $5 \mathrm{~min}$. To the solution was added dropwise 25 (345 $\mathrm{mg}, 1.00 \mathrm{mmol}$ ), and the reaction mixture was stirred at $-60{ }^{\circ} \mathrm{C}$ (internal temperature) for $24 \mathrm{~h}$. The reaction mixture was quenched by pouring into a vigorously stirring cold $\left(0{ }^{\circ} \mathrm{C}\right)$ saturated aqueous $\mathrm{NaHCO}_{3}$ solution $(15 \mathrm{~mL})$, and the resulting emulsion was stirred for at least $3 \mathrm{~h}$. The emulsion was filtered through a pad of Celite, and the filtrate was transferred into a $60-\mathrm{mL}$ separatory funnel. The biphasic mixture was extracted with dichloromethane $(2 \times 20 \mathrm{~mL})$, and the combined extracts were washed with brine $(10 \mathrm{~mL})$. The solution was dried over $\mathrm{Na}_{2} \mathrm{SO}_{4}$ and concentrated under reduced pressure. The resulting crude oil was chromatographed (silica gel, pentane/ether, 4/1, $30 \mathrm{~mm})$ to afford $307 \mathrm{mg}(0.81 \mathrm{mmol}$, $81 \%$ ) of $46 \mathbf{a}$ as a clear colorless oil. 


\section{Data for anti,anti-46a:}

${ }^{1}$ H NMR: $\quad\left(500 \mathrm{MHz}, \mathrm{CDCl}_{3}\right)$

7.36 - 7.26 (m, $5 \mathrm{H}, 2 \times \mathrm{HC}(7), 2 \times \mathrm{HC}(8), \mathrm{HC}(9))$ ) 4.78 (dd, $J=8.0,4.8,1 \mathrm{H}$, $\mathrm{HC}(1)) ; 3.78(\mathrm{ABX}, 2 \mathrm{H}, 2 \times \mathrm{HC}(5)) ; 3.09$ (d, $J=4.6,1 \mathrm{H}, \mathrm{HO}) ; 3.05$ (pent, $J=$ 7.6, $1 \mathrm{H}, \mathrm{HC}(2)) ; 2.89$ (sextd, $J=5.1,1.2,1 \mathrm{H}, \mathrm{HC}(4)) ; 1.04$ (m, $21 \mathrm{H}, 3$ x $\mathrm{HC}(12), 18 \times \mathrm{HC}(13)) ; 0.97$ (d, $J=7.1,3 \mathrm{H}, 3 \times \mathrm{HC}(10)) ; 0.93$ (d, $J=7.1,3 \mathrm{H}, 3$ $\mathrm{x} \mathrm{HC}(11))$

${ }^{13} \mathrm{C} \mathrm{NMR}: \quad\left(126 \mathrm{MHz}, \mathrm{CDCl}_{3}\right)$

217.9 (C(3)); 142.3 (C(6)); 128.2 (2 x C(7)); 127.1 (C(9)); 125.5 (2 x C(8)); 76.5 $(\mathrm{C}(1)) ; 65.5(\mathrm{C}(5)) ; 53.1(\mathrm{C}(2))$; $49.1(\mathrm{C}(4)) ; 17.9,17.9$ (6 x C(13)); $13.8(\mathrm{C}(10))$; $12.8(\mathrm{C}(11)) ; 11.8(3 \times \mathrm{C}(12))$

IR: (neat) 3452 (br, m); 3064 (w); 3032 (w); 2943 (s); 2893 (m); 2868 (s); 1711 (m); 1495 (w); 1462 (m); 1383 (m); 1248 (m); 1101 (m); 1066 (m); 1011 (m)

MS: $\quad(\mathrm{FI})$

$379\left(3, \mathrm{M}^{+}\right) ; 337(7) ; 336(25) ; 335$ (100); $272(4) ; 229(4) ; 58(7)$

Opt. Rot.: $\quad[\alpha]_{\mathrm{D}}^{24}+109.5(\mathrm{EtOH}, \mathrm{c}=0.68)$

TLC: $\quad R f 0.22$ (silica gel, pentane/ether, 4/1)

SFC: $\quad$ (Chiralcel-OD, $125 \mathrm{bar}, 3 \mathrm{~mL} / \mathrm{min}, 5.0 \% \mathrm{MeOH}$ ) (anti,anti)-46a $t_{R} 4.194 \min (96.5 \%)$; (anti,syn)-46a, $t_{R}=3.311 \min (1.3 \%)$; $\left(\right.$ syn, syn)-46a, $t_{R}=3.712 \min (1.4 \%) ;\left(\right.$ syn, anti)-46a, $t_{R}=4.979 \min (0.7 \%)$

Analysis: $\quad \mathrm{C}_{22} \mathrm{H}_{38} \mathrm{O}_{3} \mathrm{Si}(378.62)$
Calcd:
C, 69.79\%;
H, $10.12 \%$
Found:
C, 69.86\%;
H, $10.31 \%$ 


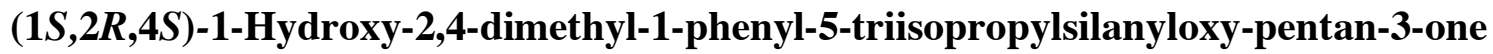
(anti,syn-46a)

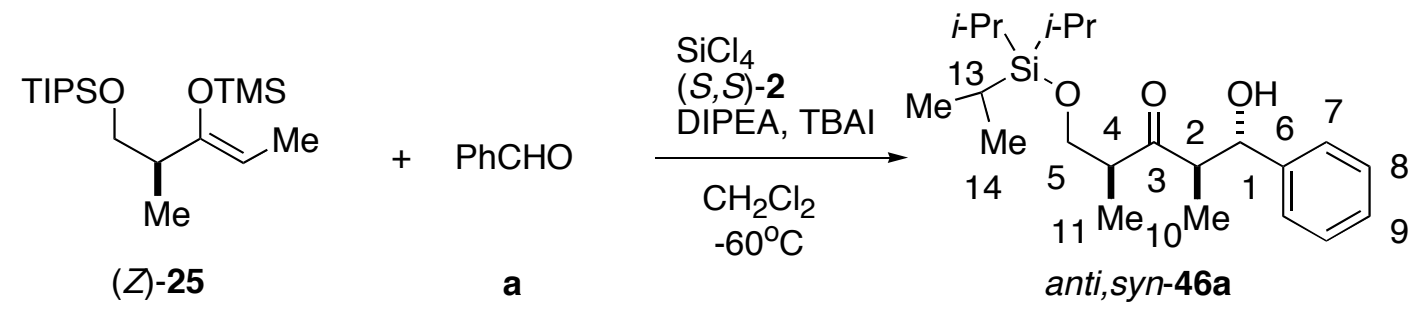

Following General Procedure IX using (Z)-25 (345 mg, $1.00 \mathrm{mmol})$, benzaldehyde (152 $\mu \mathrm{L}, \quad 1.50 \mathrm{mmol}), \quad(S, S)-2 \quad(84 \mathrm{mg}, \quad 0.10 \mathrm{mmol}), \quad \mathrm{SiCl}_{4} \quad(0.172 \mathrm{~mL}, \quad 1.50 \mathrm{mmol})$; tetrabutylammonium iodide $(74 \mathrm{mg}, 0.20 \mathrm{mmol})$ and diisopropylethylamine $(0.035 \mathrm{~mL}, 0.20$ $\mathrm{mmol})$ in $2 \mathrm{~mL}$ of $\mathrm{CH}_{2} \mathrm{Cl}_{2}$ for $24 \mathrm{~h}, 272 \mathrm{mg}(0.72 \mathrm{mmol}, 72 \%)$ of $46 \mathbf{a}$ was obtained as a clear viscous oil after column chromatography (silica gel, pentane/ether, 4/1).

Data for anti,syn-46a:

${ }^{1}$ H NMR: $\quad\left(500 \mathrm{MHz}, \mathrm{CDCl}_{3}\right)$

$7.34-7.27$ (m, $5 \mathrm{H}, 2$ x $\mathrm{HC}(7), 2 \times \mathrm{HC}(8), \mathrm{HC}(9)) ; 4.74$ (dd, $J=8.5,3.9,1 \mathrm{H}$, $\mathrm{HC}(1)) ; 3.91-3.62(\mathrm{ABX}, 2 \mathrm{H}, 2 \times \mathrm{HC}(5)) ; 3.26$ (d, $J=3.9,1 \mathrm{H}, \mathrm{HO}) ; 3.11$ (pent, $J=7.2,1 \mathrm{H}, \mathrm{HC}(2)) ; 3.04$ (sext, $J=5.9,1 \mathrm{H}, \mathrm{HC}(4)$ ); 1.06 (m, $21 \mathrm{H}, 3 \mathrm{x}$ $\mathrm{HC}(12), 18 \times \mathrm{HC}(13), 3 \times \mathrm{HC}(11)) ; 0.91$ (d, $J=7.1,3 \mathrm{H}, 3 \times \mathrm{HC}(10))$

${ }^{13} \mathrm{C} \mathrm{NMR}: \quad\left(126 \mathrm{MHz}, \mathrm{CDCl}_{3}\right)$

218.5 (C(3)); $142.0(\mathrm{C}(6)) ; 128.4(2 \times \mathrm{C}(7)) ; 127.8(\mathrm{C}(9)) ; 126.7$ (2 x C(8)); 77.1 (C(1)); 65.9 (C(5)); 53.4 (C(2)); 49.1 (C(4)); 17.9 (6 x C(13)); 14.2 (C(10)); 13.1 $(\mathrm{C}(11)) ; 11.8(3 \times \mathrm{C}(12))$

IR: (neat) 3477 (br, m); 3064 (w); 3032 (w); 2943 (s); 2891 (m); 2868 (s); 1707 (m); 1462 (m); $1385(\mathrm{~m}) ; 1250(\mathrm{~m}) ; 1101(\mathrm{~m}) ; 1068$ (m); 999 (m)

$\underline{\mathrm{MS}}: \quad(\mathrm{FI})$

379 (4); 337 (9); 336 (27); 335 (100); 272 (7); 229 (3); 58 (5)

Opt. Rot.: $\quad[\alpha]_{D}^{24}-47.1(\mathrm{EtOH}, \mathrm{c}=0.83)$

TLC: $\quad R f 0.33$ (silica gel, pentane/ether, 4/1)

SFC: $\quad$ (Chiralcel-OD, 125 bar, $3 \mathrm{~mL} / \mathrm{min}, 5.0 \% \mathrm{MeOH}$ ) 
(anti,syn)-46a, $t_{R}=3.262 \min (82.6 \%) ;\left(\right.$ anti,anti)-46a $t_{R}=4.195 \min (14.8 \%)$; (syn,anti)-46a, $t_{R}=4.956 \min (1.6 \%)$

Analysis: $\quad \mathrm{C}_{22} \mathrm{H}_{38} \mathrm{O}_{3} \mathrm{Si}(378.62)$

Calcd: $\quad$ C, $69.79 \% ; \quad H, 10.12 \%$

Found: $\quad$ C, $69.90 \% ; \quad H, 10.49 \%$

\section{(3S,1'R,2'S)- 3-Triisopropylsilanyloxybutyric acid 2-Hydroxy-1-methyl-2-phenyl-ethyl} ester (49)

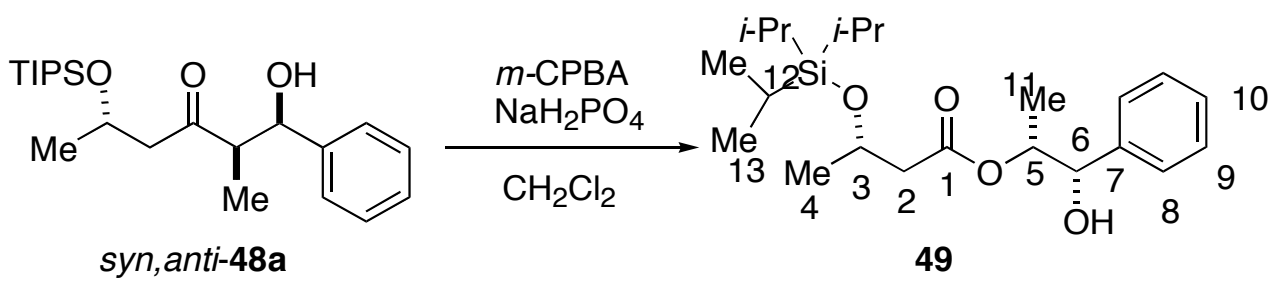

In a 10-mL Schlenk flask was placed m-CPBA (393 mg, $2.28 \mathrm{mmol}, 4.0$ equiv) in dichloromethane $(3 \mathrm{~mL})$. To the solution were added $\mathrm{NaH}_{2} \mathrm{PO}_{4}$ (486 mg, $3.42 \mathrm{mmol}$, 6.0 equiv) and a solution of $48 \mathbf{a}(215 \mathrm{mg}, 0.57 \mathrm{mmol})$ in dichloromethane $(0.5 \mathrm{~mL})$. The reaction mixture was stirred at $\mathrm{rt}$ for $48 \mathrm{~h}$. The reaction mixture was diluted in dichloromethane $(15 \mathrm{~mL})$, poured into a 60-mL separatory funnel, and washed sequentially with saturated aqueous $\mathrm{NaHCO}_{3}$ solution $(10 \mathrm{~mL})$ and with saturated aqueous $\mathrm{Na}_{2} \mathrm{SO}_{3}$ solution $(2 \times 10 \mathrm{~mL})$. The solution was dried over $\mathrm{Na}_{2} \mathrm{SO}_{4}$ and concentrated under reduced pressure. The crude NMR showed ca. $60 \%$ conversion. The crude oil was chromatographed (silica gel, hexanes/ether, 4/1, $30 \mathrm{~mm}$ ) to afford $115 \mathrm{mg}(0.29 \mathrm{mmol}, 51 \%)$ of 49 and $75 \mathrm{mg}(0.20 \mathrm{mmol}, 34 \%)$ of recovered $48 \mathrm{a}$.

\section{Data for 49:}

${ }^{1} \mathrm{H}$ NMR: $\quad\left(500 \mathrm{MHz}, \mathrm{CDCl}_{3}\right)$

$7.39-7.28$ (m, $5 \mathrm{H}, 2 \times \mathrm{HC}(8), 2 \times \mathrm{HC}(9), \mathrm{HC}(10)) ; 5.14$ (qd, $J=6.3,2.5,1 \mathrm{H}$, $\mathrm{HC}(5)) ; 4.86$ (t, $J=3.6,1 \mathrm{H}, \mathrm{HC}(6)) ; 4.35$ (sext, $J=6.8,1 \mathrm{H}, \mathrm{HC}(3)) ; 2.48$ (ABX, $\left.J_{\mathrm{AB}}=14.9,2 \mathrm{H}, 2 \times \mathrm{HC}(2)\right) ; 2.25(\mathrm{~d}, J=2.9,1 \mathrm{H}, \mathrm{OH}) ; 1.19(\mathrm{~d}, J=6.1,3 \mathrm{H}, 3 \mathrm{x}$ $\mathrm{HC}(11)) ; 1.15$ (d, $J=6.6,3 \mathrm{H}, 3 \times \mathrm{HC}(4)) ; 1.06$ (m, $21 \mathrm{H}, 18 \times \mathrm{HC}(13), 3 \times$ $\mathrm{HC}(12)$ 
${ }^{13} \mathrm{C}$ NMR: $\quad\left(126 \mathrm{MHz}, \mathrm{CDCl}_{3}\right)$

$171.1(\mathrm{C}(1)) ; 139.8(\mathrm{C}(7)) ; 128.3(2 \times \mathrm{C}(9)) ; 127.8$ (C(10)); $126.4(2 \times \mathrm{C}(8)) ; 75.5$ $(\mathrm{C}(5)) ; 75.4(\mathrm{C}(6)) ; 74.1(\mathrm{C}(3)) ; 45.0(\mathrm{C}(2)) ; 23.7$ (C(4)); 18.0, 18.0 (6 x C(13)); $13.8(\mathrm{C}(11)) ; 12.3(3 \times \mathrm{C}(12))$

IR: (neat)

3470 (br, m); 3065 (w); 3031 (w); 2944 (s); 2893 (m); 2867 (s); 2724 (w); 1736 (s); 1604 (w); 1495 (w); 1455 (m); 1379 (m); 1300 (m); 1248 (m); 1184 (s); 1128 (m); 1099 (m); $1070(\mathrm{~m}) ; 1008$ (m)

MS: $\quad(\mathrm{FI})$

$395\left(5, \mathrm{M}^{+}\right) ; 351(100)$

Opt. Rot.: $\quad[\alpha]_{\mathrm{D}}^{24}+23.2(\mathrm{EtOH}, \mathrm{c}=0.12)$

TLC: $\quad R f 0.12$ (silica gel, hexanes/ether, 4/1)

Analysis: $\quad \mathrm{C}_{22} \mathrm{H}_{38} \mathrm{O}_{4} \mathrm{Si}$ (394.62)
Calcd:
C, 66.96\%;
$\mathrm{H}, 9.71 \%$
Found:
C, 67.05\%;
$\mathrm{H}, 9.66 \%$

\section{(1S,2R)-1-Phenyl-propane-1,2-diol (50)}

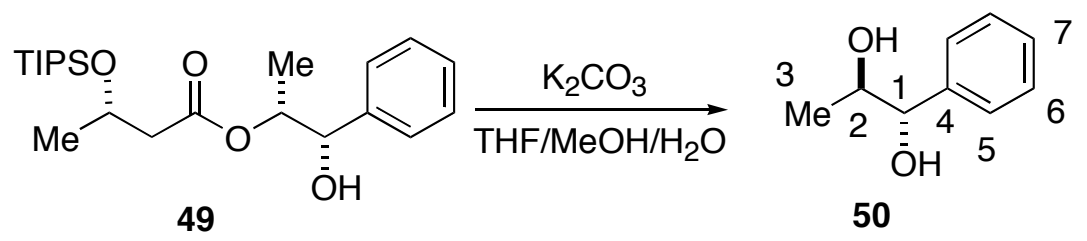

In an NMR tube was placed $49(50 \mathrm{mg}, 0.13 \mathrm{mmol})$ in $d_{8}$-THF $(0.75 \mathrm{~mL}) / d_{4}-\mathrm{MeOH}(0.75$ $\mathrm{mL}) / \mathrm{D}_{2} \mathrm{O}(0.3 \mathrm{~mL})$. To the solution was added $\mathrm{K}_{2} \mathrm{CO}_{3}(36 \mathrm{mg}, 0.26 \mathrm{mmol}, 2.0$ equiv), and the reaction mixture was thoroughly mixed. The reaction mixture was let stand at $\mathrm{rt}$ for $24 \mathrm{~h}$. The reaction mixture was transferred to a $25-\mathrm{mL}$ round-bottom flask, and the volatiles were removed under reduced pressure. The residue was chromatographed (silica gel, pentane/ether, 1/1) to afford $18 \mathrm{mg}(0.12 \mathrm{mmol}, 91 \%)$ of $\mathbf{5 0}$ as clear colorless oil. The physical properties of $\mathbf{5 0}$ were consistent with the literature. ${ }^{11}$ 


\section{Data for 50:}

${ }^{1}$ H NMR: $\quad\left(500 \mathrm{MHz}, \mathrm{CDCl}_{3}\right)$

$7.27-7.16$ (m, $5 \mathrm{H}$, aromatic); 4.58 (d, $J=4.9,1 \mathrm{H}, \mathrm{HC}(1)) ; 3.92(\mathrm{qd}, J=6.5$, 4.8, $1 \mathrm{H}, \mathrm{HC}(2)) ; 0.99$ (d, $\left.J=6.0,3 \mathrm{H}, \mathrm{H}_{3} \mathrm{C}(3)\right)$

${ }^{13} \mathrm{C} \mathrm{NMR}: \quad\left(126 \mathrm{MHz}, \mathrm{CDCl}_{3}\right)$

$140.3(\mathrm{C}(4)), 128.4(\mathrm{C}(6)), 127.9$ (C(7)), $126.6 \quad(\mathrm{C}(5)) ; 77.5 \quad(\mathrm{C}(1)) ; 71.3$ $(\mathrm{C}(2)) ; 17.3(\mathrm{C}(3))$

IR: (neat)

3392 (br, s); 3064 (w); 3031 (m); 2978 (s); 2932 (m); 1708 (w); 1604 (w); 1494 (m); 1453 (m); 1377 (m); 1201 (m); 1130 (m); 1079 (m)

Opt. Rot.: $\quad[\alpha]_{\mathrm{D}}^{24}+14.7(\mathrm{EtOH}, \mathrm{c}=1.2)\left(\right.$ Lit. for $(1 S, 2 R)-50[\alpha]_{\mathrm{D}}^{24}+17.2(\mathrm{EtOH}, \mathrm{c}=4.2) .{ }^{11}$

\section{References}

(1) Gilman, H.; Cartledge, F. K. J. Organometal. Chem. 1964, 2, 447.

(2) Still, W. C.; Kahn, M.; Mitra, A. J. Org. Chem. 1978, 43, 2923.

(3) Hall, P. L.; Gilchrist, J. H.; Collum, D. B. J. Am. Chem. Soc., 1991, 113, 9571.

(4) Prakash, C.; Saleh, S.; Blair, L. A. Tetrahedron Lett. 1989, 39, 19-22.

(5) Martin, V. A.; Murray, D. H.; Pratt, N. E.; Zhao, Y.; Albizati, K. F. J. Am. Chem. Soc. 1990, 112, 6965-6968.

(6) Taniguchi, Y.; Inanaga, J.; Yamaguchi, M. Bull. Chem. Soc. Jpn. 1981, 54, 32293232.

(7) Busch-Petersen, J.; Corey, E. J. Tetrahedron Lett., 2000, 41, 2515.

(8) Martin, V. A.; Murray, D. H.; Pratt, N. E.; Zhao, Y.-B.; Albizati, K. F. J. Am. Chem. Soc. 1990, 112, 6965-6978.

(9) Denmark, S. E.; Pham. S. M. Org. Lett. 2001, 3, 2201.

(10) Galobardes, M.; Gascón, M.; Mena, M.; Romea, P.; Urpí, F.; Vilarrasa, J. Org. Lett. 2000, 2, 2599.

(11) Kihumbu, D.; Stillger, T.; Hummel, W.; Liese, A. Tetrahedron: Asymmetry 2002, 13, 1069-1072. 


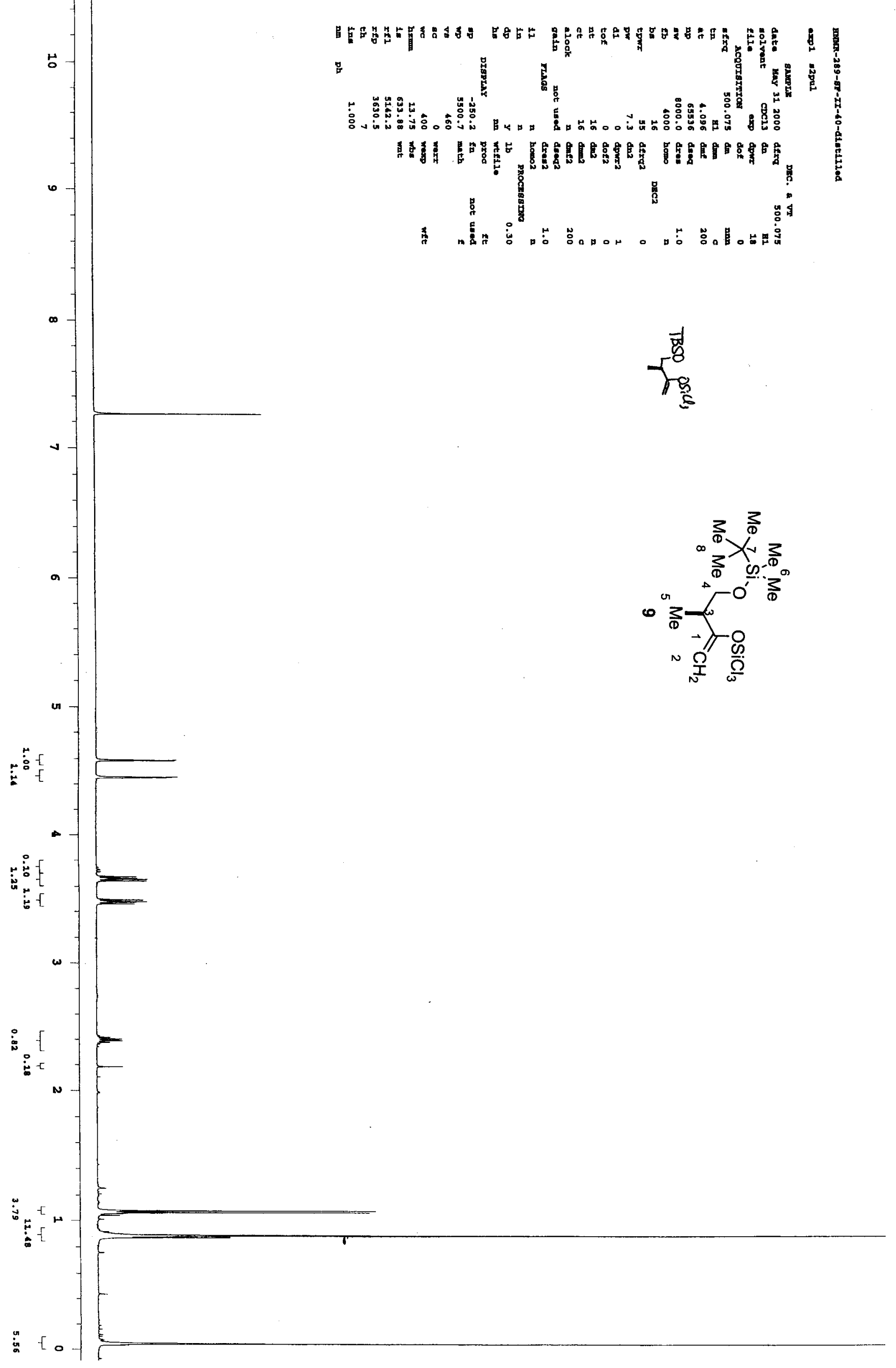




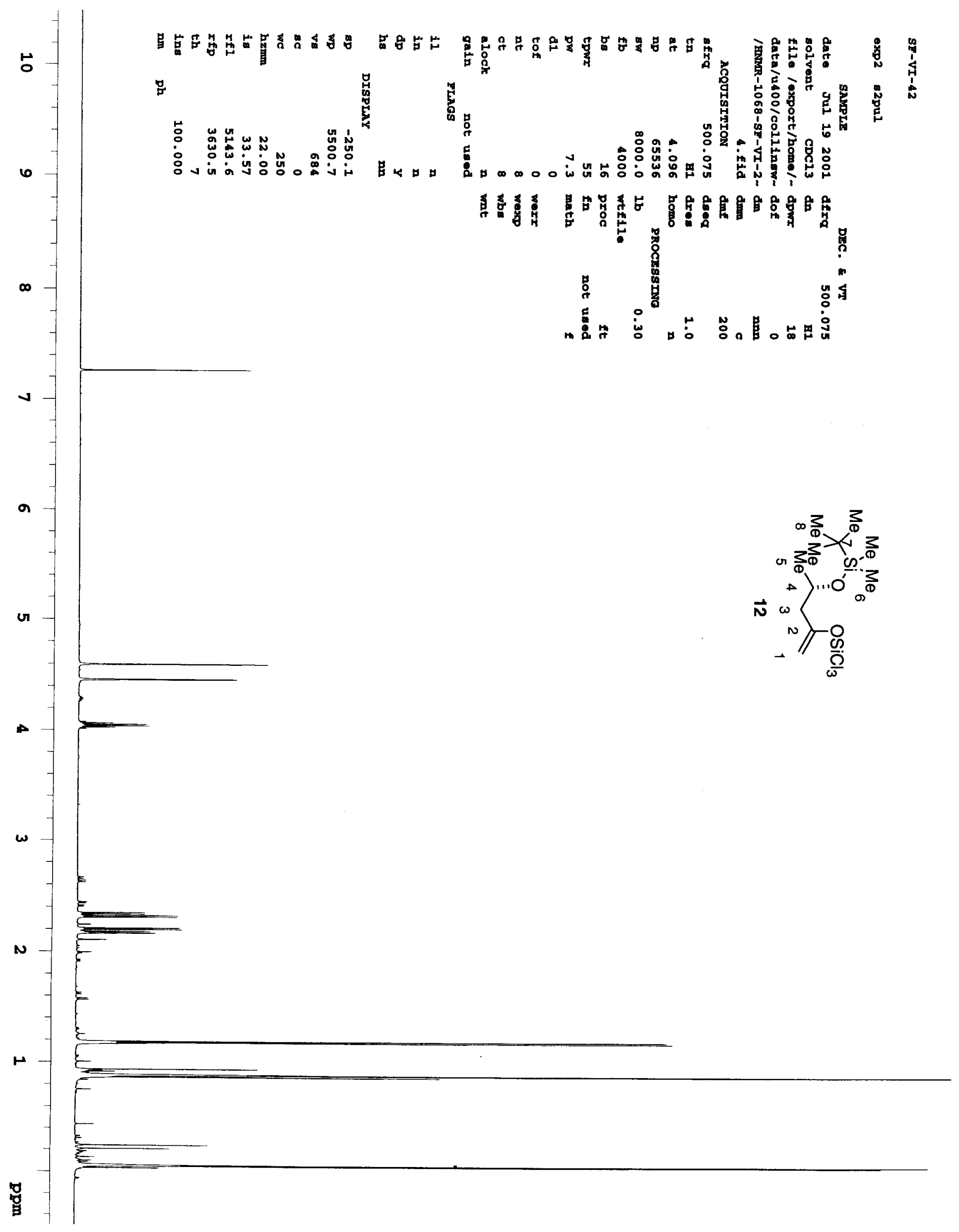




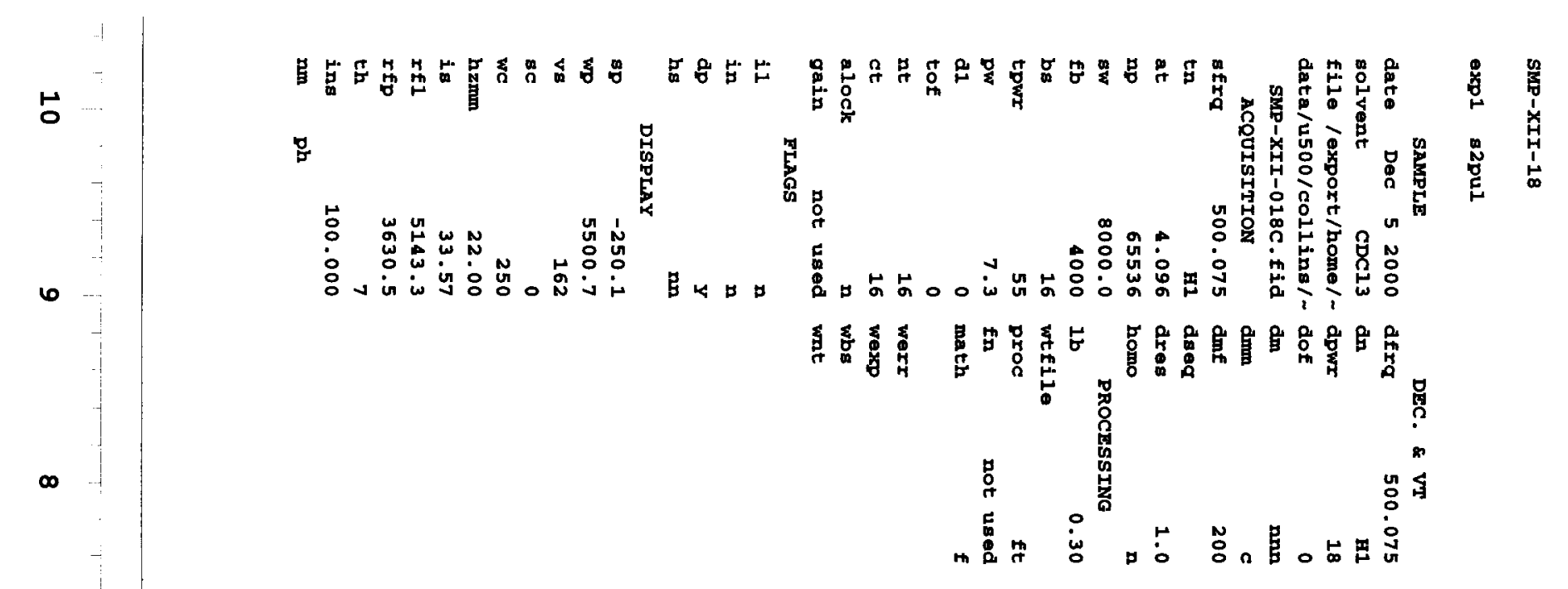

$\mathbf{1}$
a
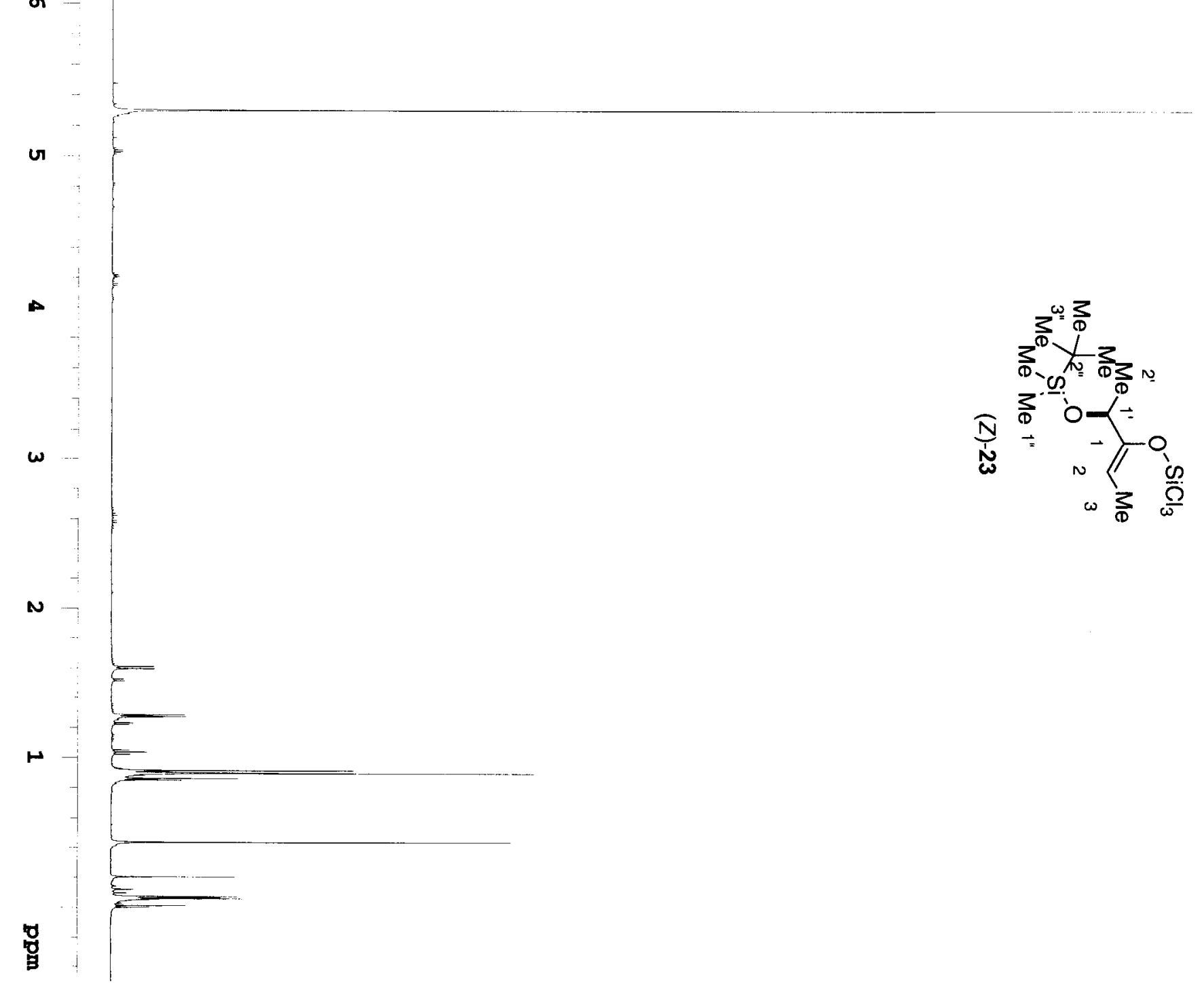


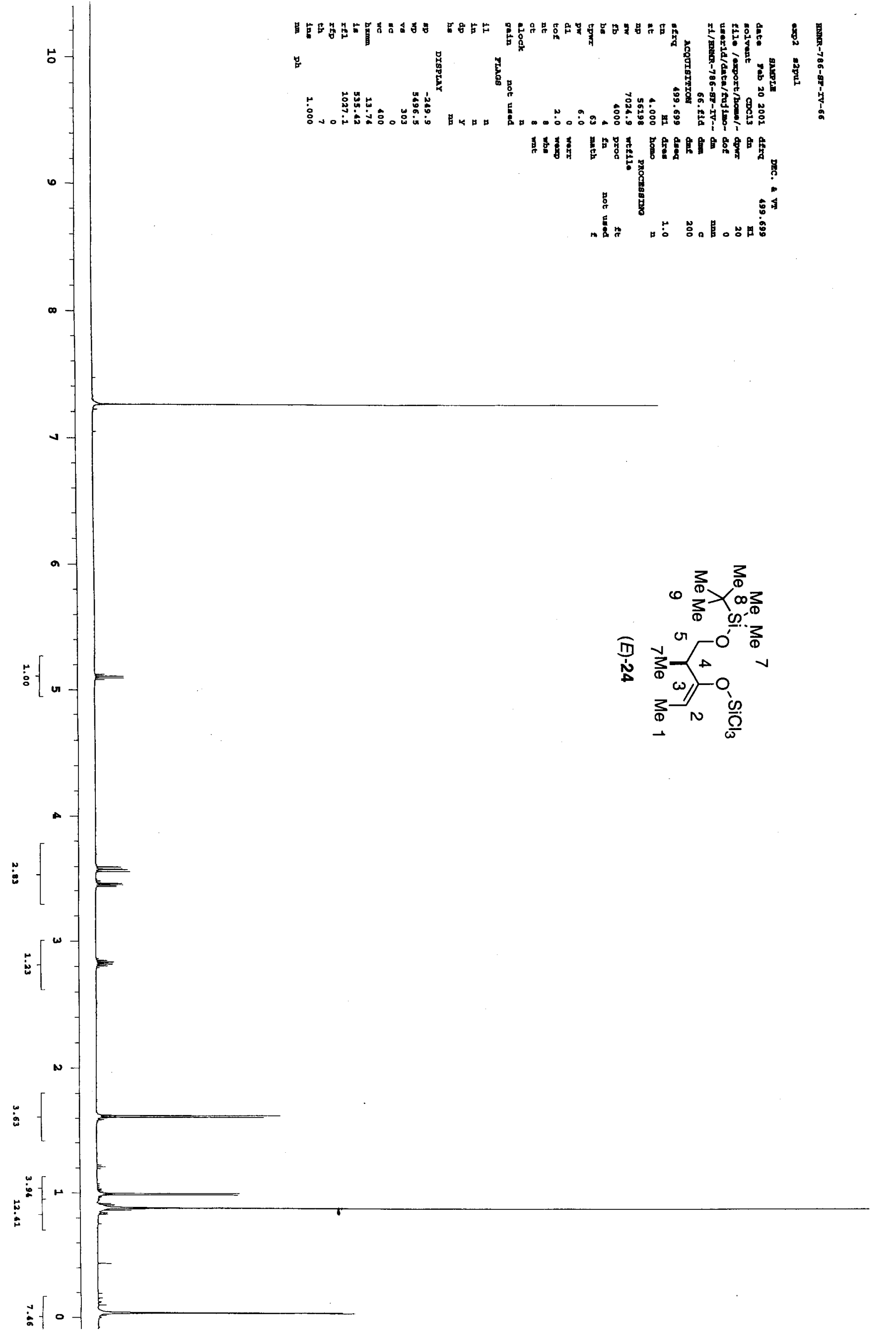




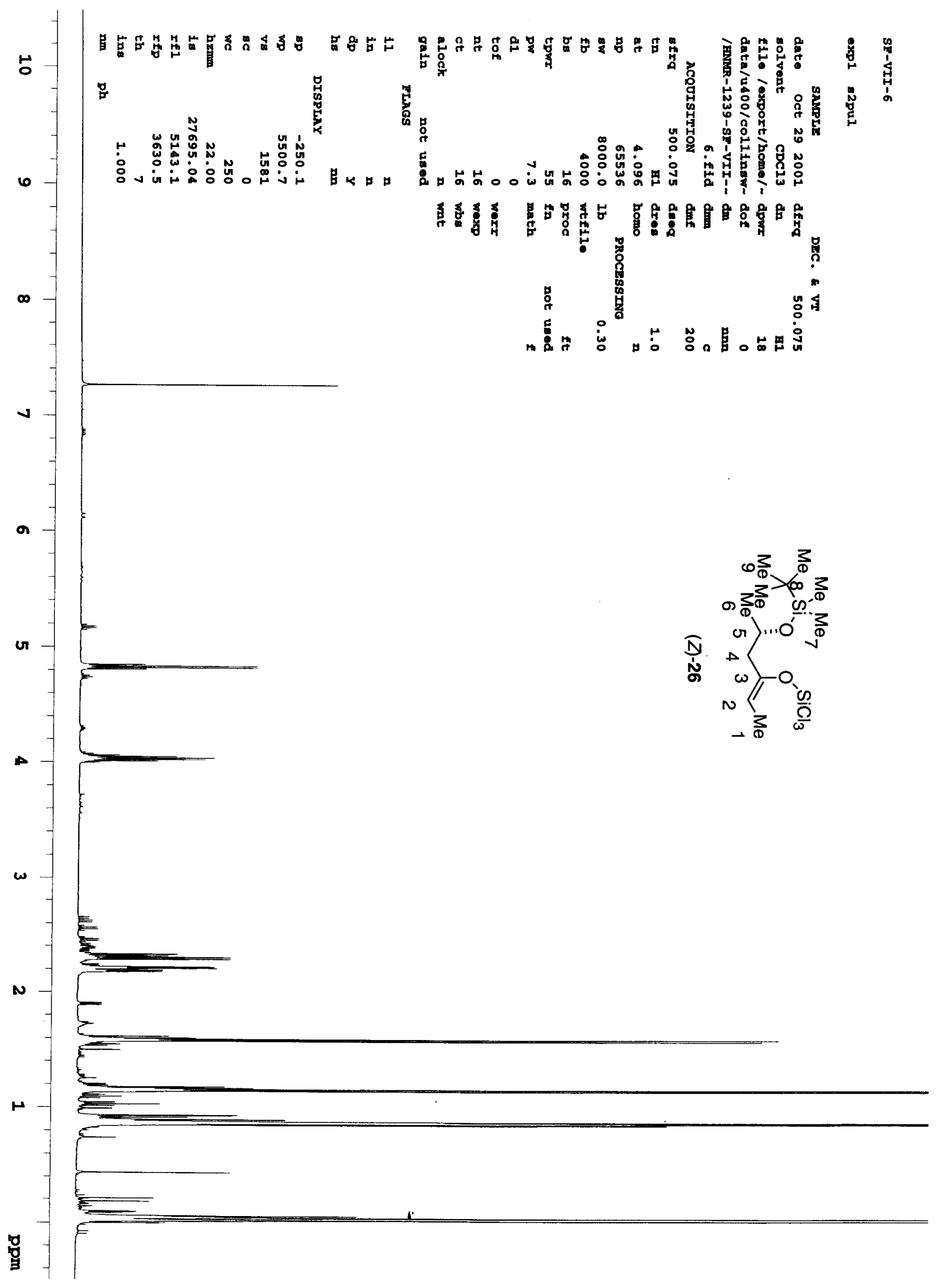




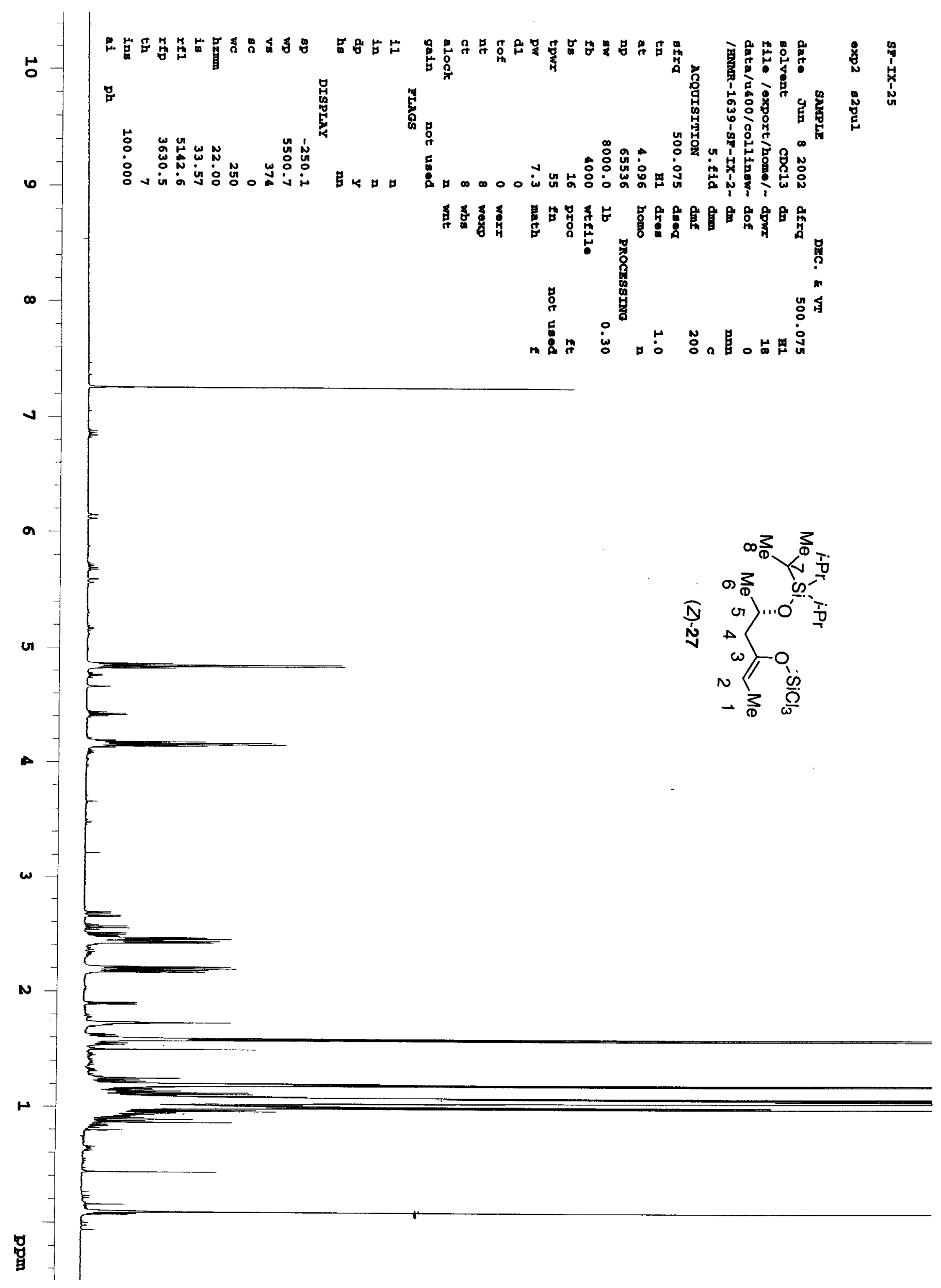




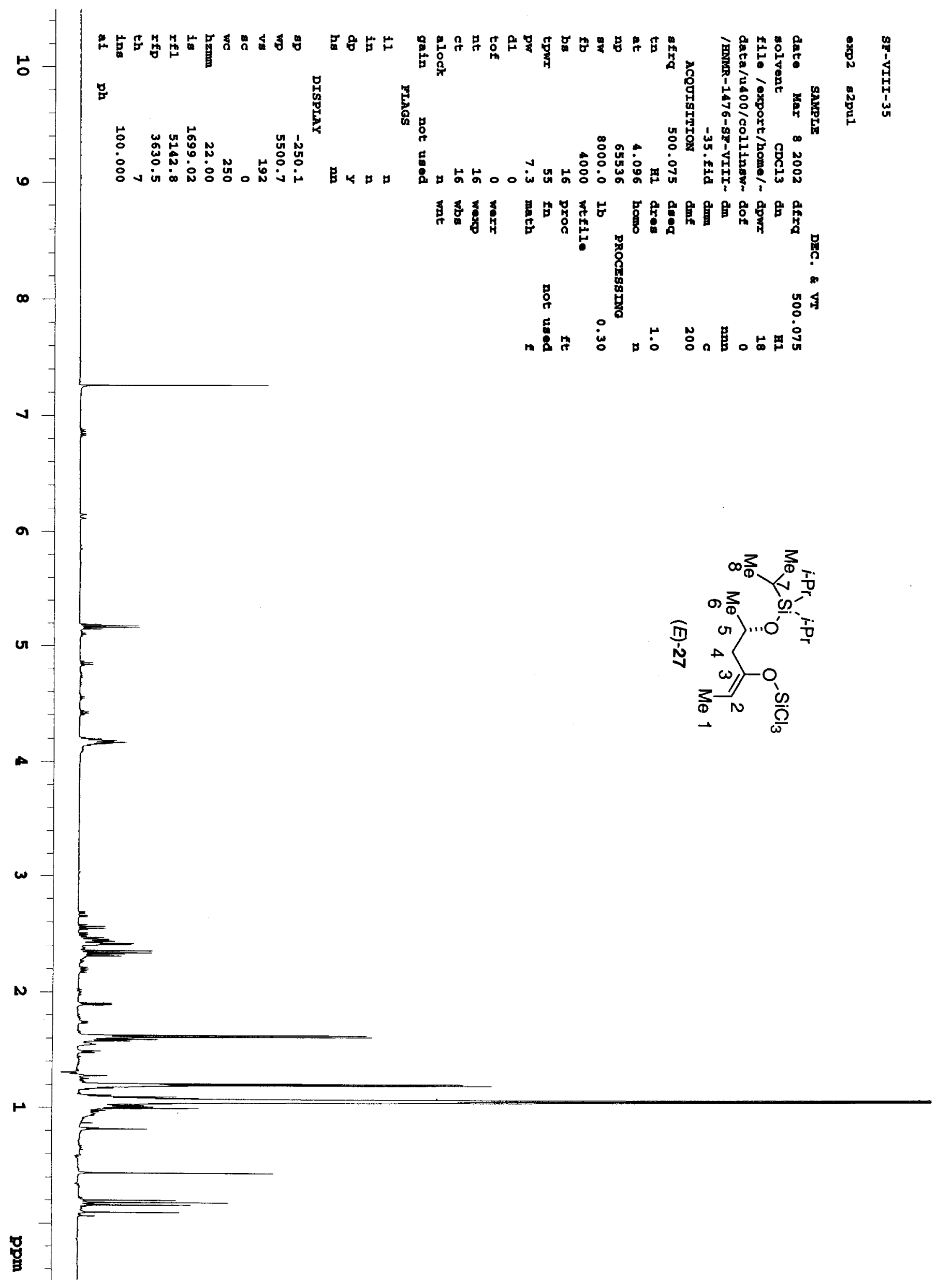

